\title{
CONTROLLING SECONDARY FLOWS IN HIGHLY-LOADED COMPRESSOR CASCADES
}

\author{
by \\ Jean-Michel Prévost \\ A thesis submitted to the Faculty of Graduate and Postdoctoral Affairs \\ in partial fulfilment of the degree requirements of
}

Master of Applied Science

in

Aerospace Engineering

Carleton University
Ottawa, Ontario, Canada

(C) 2015

Jean-Michel Prévost 


\begin{abstract}
This thesis documents the experimental results of a research program investigating the effect of non-axisymmetric endwall contouring on the secondary flows and loss generation in a highly-loaded compressor cascade. The results are compared with the losses for the baseline flat endwall in order to evaluate the potential benefits.

The compressor blade was designed by Pratt \& Whitney Aircraft and is representative of an exit guide vane row in an aircraft engine. The experimental study was conducted in Carleton University's low speed linear cascade wind tunnel. The measurements were made at a constant Reynolds number of 150,000 (based on the axial chord and the inlet velocity) for two values of incidence, $0^{\circ}$ (design incidence) and $+7^{\circ}$.

Quantitative results were obtained from static pressure measurements on the suction and pressure surfaces of the blade to evaluate the blade loadings and from measurements made by a seven-hole pressure probe downstream of the cascade to assess the blade row losses. Qualitative results were obtained from oil surface flow visualisation on the endwall and on the blade surfaces and were used to assist in the interpretation of the flow physics.

The current research showed that the application of non-axisymmetric endwall contouring at design incidence modified the secondary flows near the endwall, mitigating the formation of the corner stall. The benefits from contouring were observed in terms of reductions in the secondary losses and in the underturning of the secondary flows compared to the flat endwall test case. However, these benefits were not observed at the off-design incidence studied. The application of endwall contouring generated higher secondary kinetic energy near the endwall, which penetrated deeper along the span and occupied a larger area, resulting in higher secondary and total losses as it dissipated moving downstream of the cascade.
\end{abstract}




\section{Acknowledgments}

Following the completion of my bachelor degree, I wanted to pursue my studies, but this time, doing something where I would have the freedom to think, to explore and to make discoveries. I was given this opportunity following discussions with my then-to-be supervisor on a potential project he was given by Pratt \& Whitney Aircraft. Following these discussions, I embark on a new adventure.

I would like to show my profound gratitude to my thesis supervisor, Professor Steen A. Sjolander, for giving me this opportunity. His guidance, motivation, patience and continuous support have been proved invaluable in helping me complete my work. The guidance and support received gave me the confidence and energy required to complete my work. Thank you very much for all.

This research could not have been completed without the financial support from Pratt \& Whitney Aircraft (PWA). I gratefully acknowledge their support and would like to specially thank Brian Green, who was the technical lead and principal contact for this project. His feedback and support throughout the whole project was well appreciated.

Thank you to the staff of the Department of Mechanical \& Aerospace Engineering for their continuous administrative support throughout my Masters. A would like to give a special thanks to Nancy Powell for her continuous administrative support. Other special thanks to Neil McFadyen for his support on all my computer stuff and to Kevin Sangster and Alex Proctor for bearing my presence in the shop on multiple occasions. Thanks for showing me how to use the tools and for helping me accomplished my work.

Several past and current students at Carleton University had influence over this work. Thank you to Dr. Metin Yaras, Dr. Michael Benner, Dr. Ali Mahallati, Dr. Daniel Knezevici, Dr. Farzad Taremi and Dr. Hamza Abo El Ella, PhD candidate Gordon MacIsaac and finally to Tatjana Zorić, Tania Ellison and Andrew Scribner.

I would like to give a very special thanks to my "lab roommate", Gordon MacIsaac. He gave me guidance and showed me how to operate the whole thing. More than this, he was always there to support me, share my frustration, give me guidance and help me navigate through this journey. Gord, you were in some way a mentor, but also a friend. I could not thank you enough for all the time and effort you put to help me start my research and answer all my questions. Thanks. 
I would also like to give a special thanks to my family and friends, on whom I could always count for their support. They all have accompanied me along my journey in different manners and helped me surpass myself. I could not have done it without you. Merci beaucoup tout le monde pour votre support. 


\section{Table of Contents}

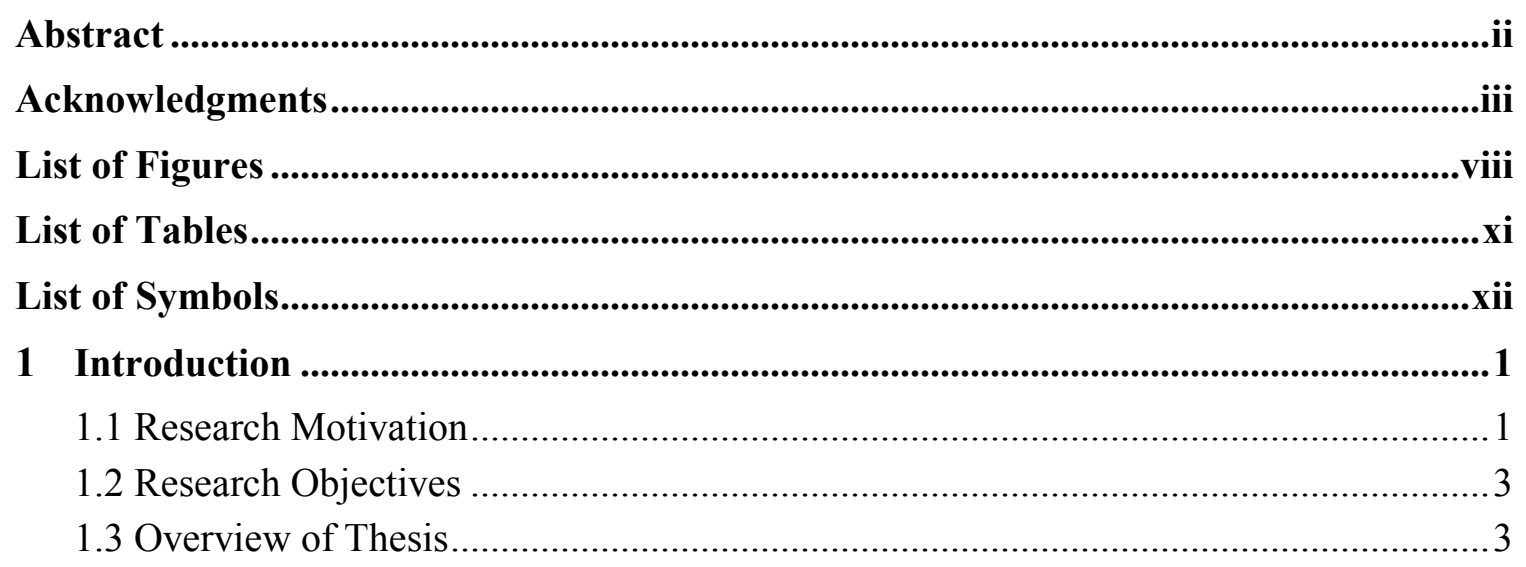

2 Background and Literature Review ...............................................................................5

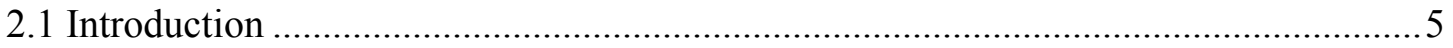

2.2 The Flow Field and Losses in Axial Turbomachinery .............................................. 5

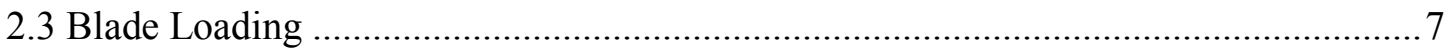

2.4 Profile and Secondary Losses in Linear Compressor Cascades .................................. 9

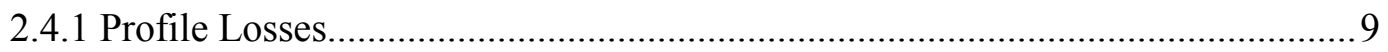

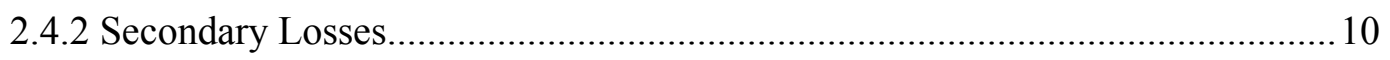

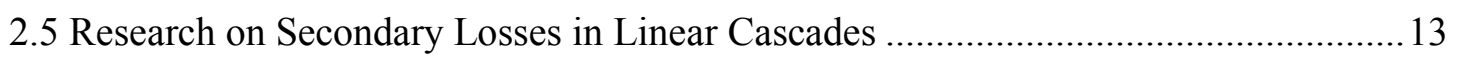

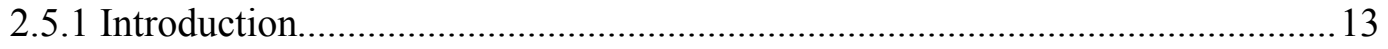

2.5.2 Effect of Inlet Boundary Layers and Freestream Turbulence Intensity............. 14

2.5.3 Effect of Blade Loadings and Blade Loading Distributions............................. 17

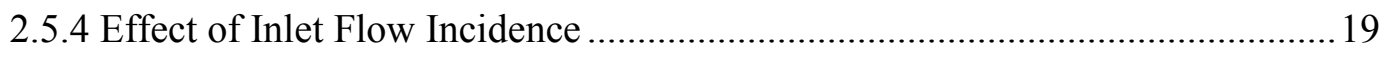

2.6 Control of Secondary Flows for Secondary Loss Mitigation ...................................20

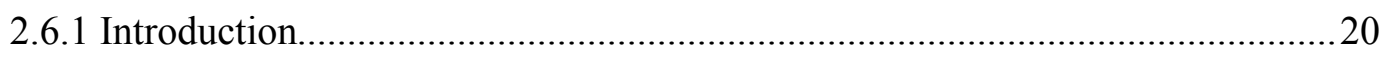

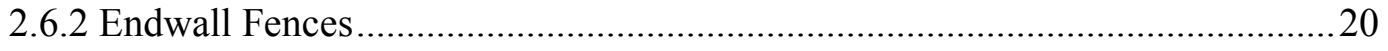

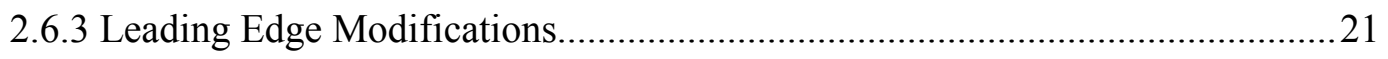

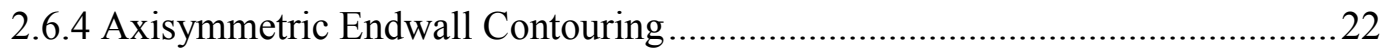

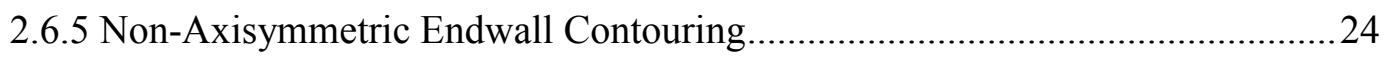

2.6.5.1 Experimental and Computational Investigations ............................... 25

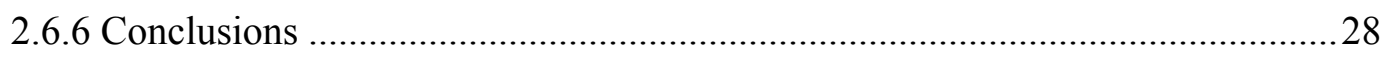

3 Experimental Methods and Data Reduction Procedures ...........................................29 


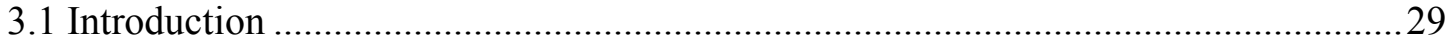

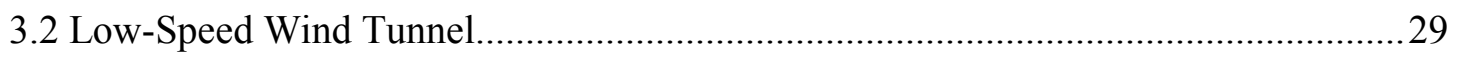

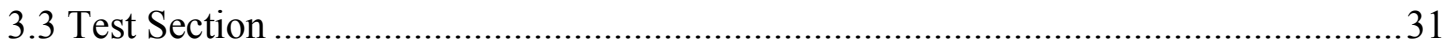

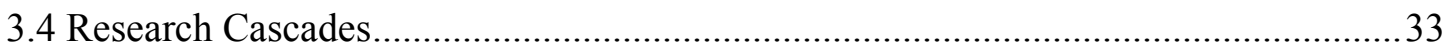

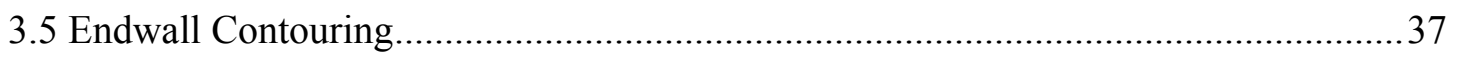

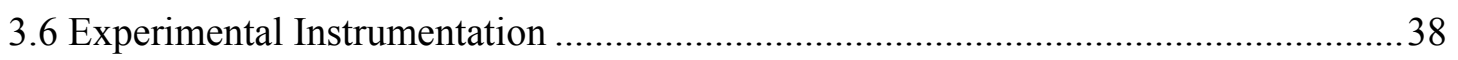

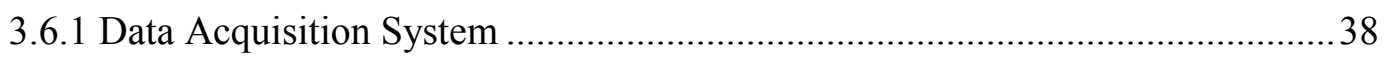

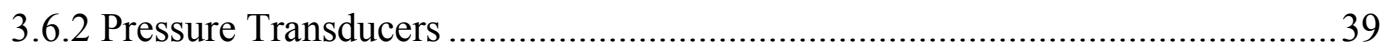

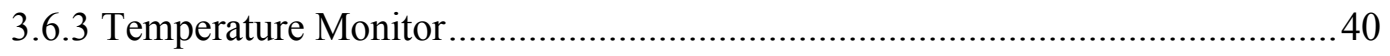

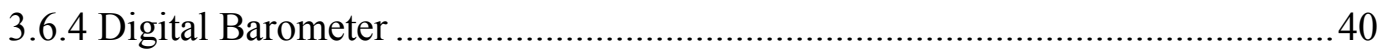

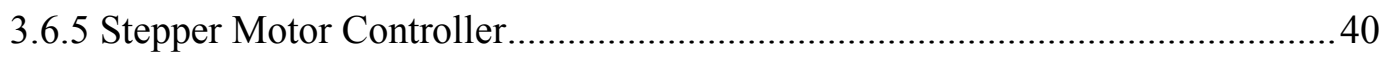

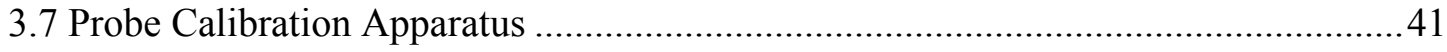

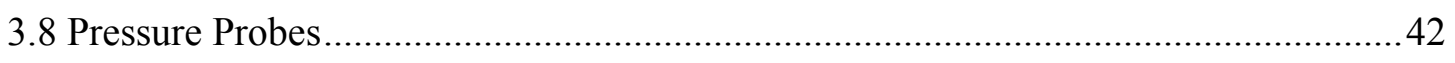

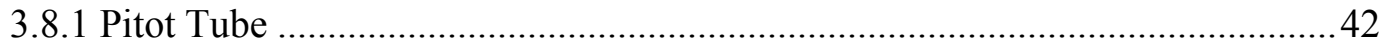

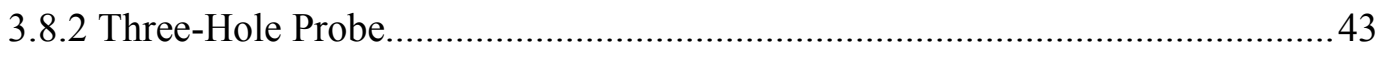

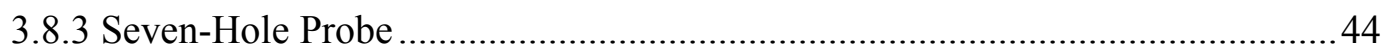

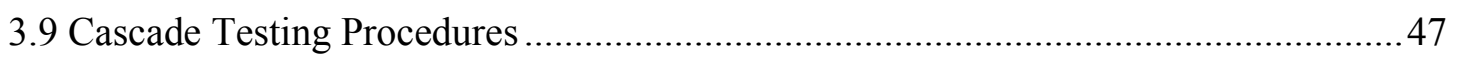

3.9.1 Wind Tunnel Calibration and Operating Point .............................................. 47

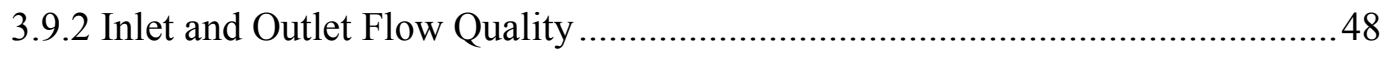

3.9.3 Seven-Hole Probe Axial Traverses.............................................................. 51

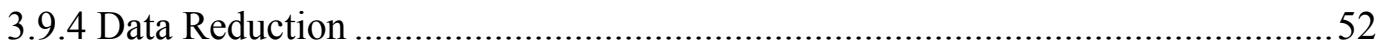

3.9.4.1 Measured and Mixed-Out Losses .................................................... 52

3.9.4.2 Streamwise Vorticity and Secondary Kinetic Energy .......................... 55

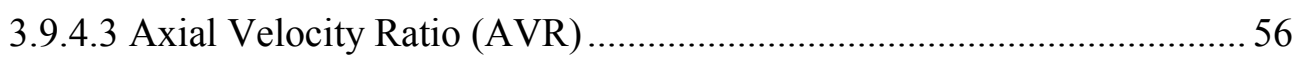

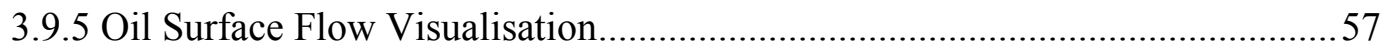

4 Secondary Flows at Design Incidence ...................................................................59

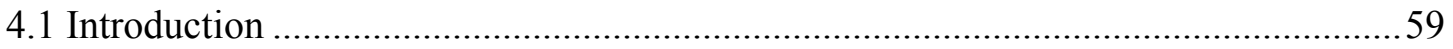

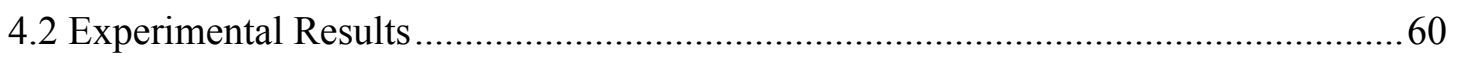

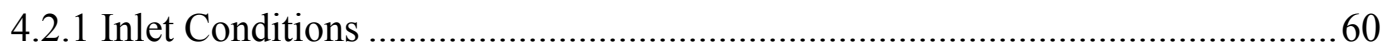

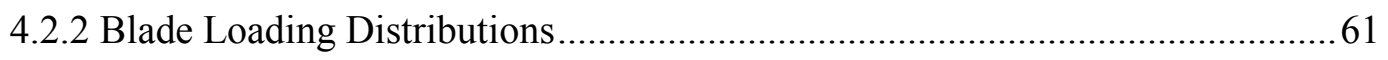

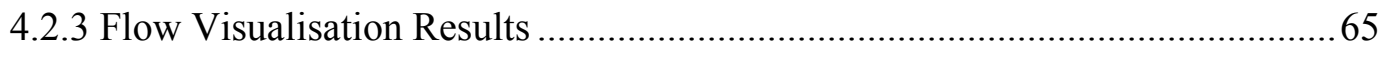

4.2.4 Downstream Flow Field Measurements ................................................... 70

4.2.4.1 Total Pressure Distributions............................................................ 70

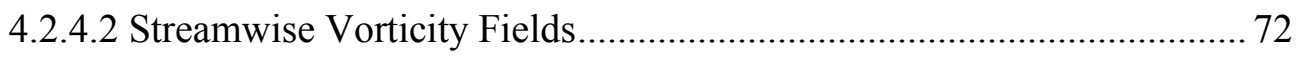

4.2.4.3 Secondary Kinetic Energy ........................................................... 75

4.2.4.4 Integrated Downstream Results .................................................... 78

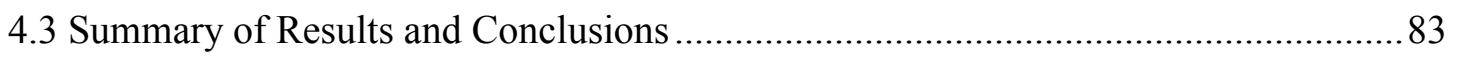

5 Secondary Flows at Off-Design Incidence ...........................................................84

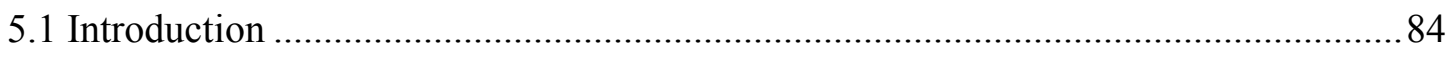




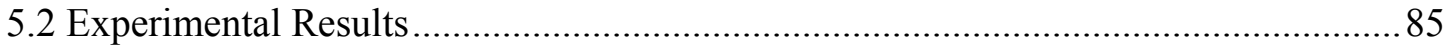

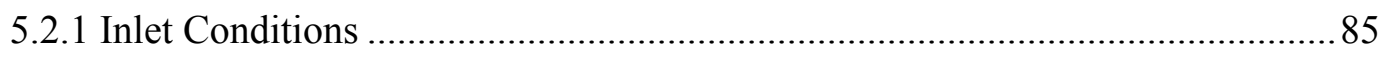

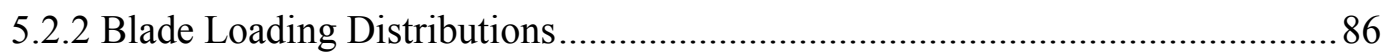

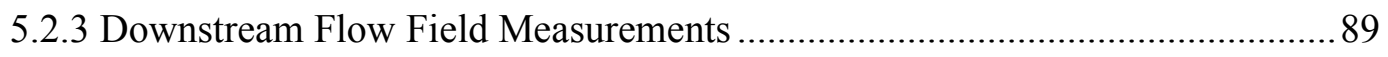

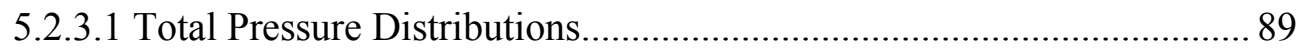

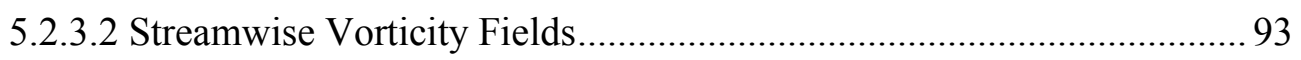

5.2.3.3 Secondary Kinetic Energy ............................................................. 98

5.2.3.4 Integrated Downstream Results ................................................... 102

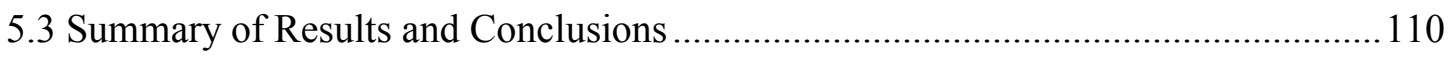

6 Conclusions and Recommendations for Future Work.................................................111

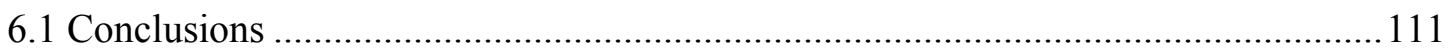

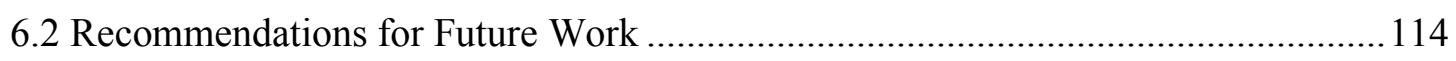

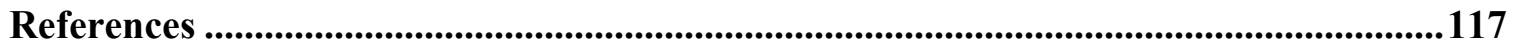

A Calibration Coefficients for Pressure Transducers...................................................124

B Calibration and Data Reduction Procedures for Three-Hole Probe .........................127

C Calibration and Data Reduction Procedures for Seven-Hole Probe ...........................131 


\section{List of Figures}

Figure 1.1 PW1500G gas turbine engine cut-away [Source: www.pw.utc.com] ............1

Figure 2.1: Flow through the blade passage of a compressor rotor (from

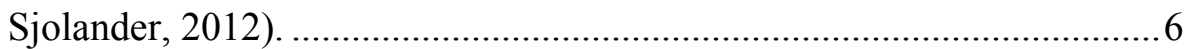

Figure 2.2: Compressor velocity distribution (adapted from Sjolander, 2012)..............7

Figure 2.3: Variation of compressor total pressure loss coefficient with diffusion factor, D (Bullock and Johnsen, 1965) ................................................ 9

Figure 2.4: Interpretation of endwall flow features (from Harvey, 2008)..................... 11

Figure 2.5: Formation of hub corner stall, from Lei et al. (2008) ................................. 13

Figure 2.6: Influence of Reynolds number and FSTI on suction surface transition onset (from Schreiber et al., 2002) ........................................................ 16

Figure 2.7: Compressor Smith chart with contours of efficiency as a function of work coefficient and flow coefficient (adapted from Dickens and Day, 2009)

Figure 3.1: Wind-tunnel schematic (adapted from Knezevici (2011) and Benner

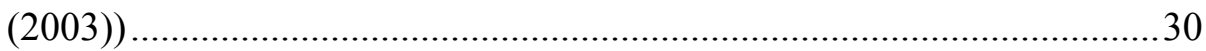

Figure 3.2: Linear cascade test section (adapted from Benner, 2003) .......................... 31

Figure 3.3 Cascade nomenclature and midspan geometry ............................................33

Figure 3.4: Variation of blade true chord along the span (Private Communication, PWA)

Figure 3.5: Rendering of CEGV-1 contoured endwall with major geometric

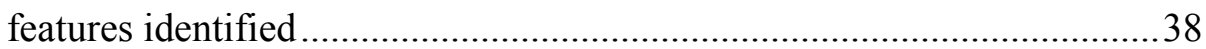

Figure 3.6 Schematic of probe calibration apparatus (from Benner, 2003) ...................42

Figure 3.7: Three-hole probe (adapted from Mahallati, 2003) ....................................44

Figure 3.8: Seven-hole probe geometry (adapted from Knezevici, 2011) .....................45

Figure 3.9: Inlet flow uniformity and outlet flow periodicity - flat endwall ..................49

Figure 3.10: Inlet boundary layer profile measured at $-1.2 \mathrm{~B}_{\mathrm{x}}$ and increments of

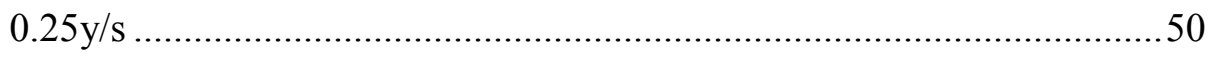

Figure 3.11: Measurement grid with trailing edge projection ...................................51 
Figure 4.1: Spanwise variation of total pressure coefficient at design incidence measured with a Pitot probe $1.2 \mathrm{Bx}$ upstream of the leading edge and $\mathrm{y} / \mathrm{s}=0.5$

Figure 4.2: Blade surface static pressure distribution at $\mathrm{z} / \mathrm{h}=0.50(\mathrm{a}), \mathrm{z} / \mathrm{h}=0.20(\mathrm{~b})$ and $\mathrm{z} / \mathrm{h}=0.5$ (c) at design incidence.

Figure 4.3: Pitchwise inlet flow angle deviation from the design inlet angle $\beta_{1, \text { design }}$ measured at $1.2 \mathrm{Bx}$ upstream of the blade leading edge and at $\mathrm{y} / \mathrm{s}=0.5$

Figure 4.4: Endwall and suction surface oil surface flow visualisations for the flat $(a, b)$ and contoured (c, d) endwall configurations at design incidence.

Figure 4.5: Total pressure deficit coefficient $\left(\mathrm{C}_{\mathrm{PO}}\right)$ floods at $1.60 \mathrm{~B}_{\mathrm{x}}$ for the flat (a) and contoured (b) endwall configurations at design incidence

Figure 4.6: Streamwise vorticity coefficient $\left(\mathrm{C}_{\mathrm{s}}\right)$ floods overlaid with secondary velocity vectors for the flat (a) and contoured (b) endwall configurations at design incidence

Figure 4.7: Line contours of total pressure deficit coefficient superimposed on flood contours of secondary kinetic energy for the flat (a) and contoured (b) endwall configurations at design incidence.

Figure 4.8: Measured pitchwise mass- and area-averaged results at $160 \% \mathrm{~B}_{\mathrm{x}}$ plane: mass-averaged total pressure loss coefficient (a), areaaveraged outlet flow angle (b) and mass-averaged secondary kinetic energy coefficient (c) at design incidence.

Figure 5.1: Blade surface static pressure distribution at $\mathrm{z} / \mathrm{h}=0.50(\mathrm{a}), \mathrm{z} / \mathrm{h}=0.20$ (b) and $\mathrm{z} / \mathrm{h}=0.05$ (c) at design and $7^{\circ}$ incidences

Figure 5.2: Total pressure deficit coefficient $\left(\mathrm{C}_{\mathrm{PO}}\right)$ floods at $1.60 \mathrm{~B}_{\mathrm{x}}$ for the flat (left) and contoured (right) endwall configurations at design (a) and $7^{\circ}$ (b) incidences.

Figure 5.3: Total pressure deficit coefficient $\left(\mathrm{C}_{\mathrm{PO}}\right)$ floods with straightened wakes for the flat (a) and contoured (b) endwall configurations at $7^{\circ}$ of incidence.

Figure 5.4: Streamwise vorticity coefficient $\left(\mathrm{C}_{\mathrm{s}}\right)$ floods overlaid with secondary velocity vectors for the flat (left) and contoured (right) endwalls at design (a) and $7^{\circ}(\mathrm{b})$ incidences

Figure 5.5: Line contours of total pressure deficit coefficient superimposed on flood contours of secondary kinetic energy for the flat (left) and ix 
contoured (right) endwall configurations at design (a) and $7^{\circ}$ (b)

incidences.....

Figure 5.6: Measured pitchwise mass- and area-averaged results at $160 \% \mathrm{~B}_{\mathrm{x}}$ plane: mass-averaged total pressure loss coefficient (a), areaaveraged outlet flow angle (b) and mass-averaged secondary kinetic energy coefficient (c) at $7^{\circ}$ incidence. 103

Figure 5.7: Summary of measured and mixed-out losses at design and $7^{\circ}$ incidences for the flat and contoured endwalls 105

Figure 5.8: Influence of incidence on measured and mixed-out total-pressure losses for the flat and contoured endwalls 109

Figure A.1: Sample calibration curves for DRAL501DN pressure transducers 125

Figure A.2: Sample calibration curves for DRAL505DN pressure transducers

Figure B.1: Three-hole probe calibration curves.... 129

Figure C.1: Division of angular space (reproduced from Gerner et al. (1984))

Figure C.2: Probe Tangential Coordinate System (adapted from Gerner et al., 1984). 133

Figure C.3: $C_{\alpha}$ calibration data for sector 7 at $15 \mathrm{~m} / \mathrm{s}$ 135

Figure C.4: $C_{\beta}$ calibration data for sector 7 at $15 \mathrm{~m} / \mathrm{s}$ 136

Figure C.5: $C_{0}$ calibration data for sector 7 at $15 \mathrm{~m} / \mathrm{s}$ 137

Figure C.6: $C_{q}$ calibration data for sector 7 at $15 \mathrm{~m} / \mathrm{s}$ 138

Figure C.7: Probe Polar Coordinate System, adapted from Gerner et al. (1984) 140

Figure C.8: $C_{\phi}$ calibration data for sector 4 at $15 \mathrm{~m} / \mathrm{s}$ 142

Figure C.9: $C_{\theta}$ calibration data for sector 4 at $15 \mathrm{~m} / \mathrm{s}$ 143

Figure C.10: $C_{0}$ calibration data for sector 4 at $15 \mathrm{~m} / \mathrm{s}$ 144

Figure C.11: $C_{q}$ calibration data for sector 4 at $15 \mathrm{~m} / \mathrm{s}$ 145 


\section{List of Tables}

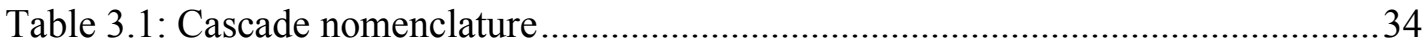

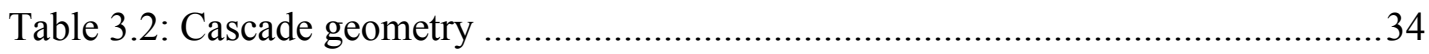

Table 4.1: Summary of experimental results at design incidence measured $1.2 \mathrm{~B}_{\mathrm{x}}$ upstream of the blade leading edge..................................................6 61

Table 4.2: Summary of experimental results at design incidence ................................. 80

Table 5.1: Summary of experimental results at $7^{\circ}$ of incidence measured $1.2 \mathrm{~B}_{\mathrm{x}}$ upstream of the blade leading edge ..................................................... 85

Table 5.2: Summary of experimental results at 7 degrees of incidence .......................... 104

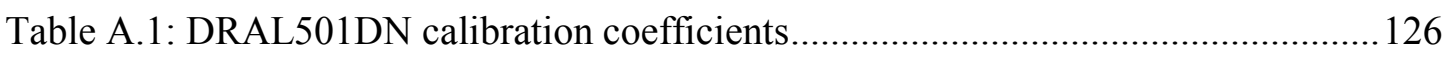

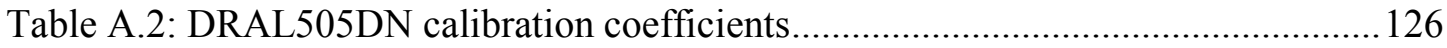




\section{List of Symbols}

\begin{tabular}{|c|c|}
\hline$A$ & Area \\
\hline$A V R$ & Axial velocity ratio \\
\hline$B$ & True chord \\
\hline$B_{x}$ & Axial chord \\
\hline$C$ & Absolute velocity \\
\hline$C_{P 0}$ & Total pressure coefficient \\
\hline$C_{P S}$ & Static pressure coefficient \\
\hline$C_{P S, b l}$ & Blade loading static pressure coefficient \\
\hline$C_{q}$ & Dynamic pressure coefficient \\
\hline$C_{S K E}$ & Secondary kinetic energy coefficient \\
\hline$C_{\omega s}$ & Streamwise vorticity coefficient \\
\hline$d$ & Probe tip diameter \\
\hline$D$ & Diffusion factor \\
\hline Hor $H_{S F}$ & Shape factor \\
\hline$h$ & Span \\
\hline$h_{0}$ & Stagnation enthalpy \\
\hline$i$ & Incidence \\
\hline$\dot{m}$ & Mass flow rate \\
\hline$P_{0}$ & Total pressure \\
\hline$P$ & Static pressure \\
\hline$q$ & Dynamic pressure, $0.5 \rho U^{2}$ \\
\hline $\operatorname{Re}$ & Reynolds number, $\tilde{U}_{C L, 1} C_{x} / v_{L}$ \\
\hline$s$ & Pitchwise spacing between adjacent blades \\
\hline$V_{i}$ & Velocity vector \\
\hline$U$ & Blade circumferential speed \\
\hline$u, v, w$ & Cartesian velocity components \\
\hline
\end{tabular}




$\begin{array}{ll}x, y, z & \text { Axial, pitchwise and spanwise directions } \\ \alpha & \text { Yaw or spanwise flow angle (measured from tangential direction) } \\ \beta & \text { Pitch or pitchwise flow angle (measured from axial direction) } \\ \delta & \text { Boundary-layer thickness or deviation angle } \\ \delta^{*} & \text { Boundary-layer displacement thickness } \\ \zeta & \text { Stagger angle (measured from axial direction to pressure side tangent) } \\ \mu & \text { Dynamic viscosity } \\ v & \text { Kinematic viscosity } \\ \rho & \text { Density } \\ \theta & \text { Boundary-layer momentum thickness } \\ \sigma & \text { Solidity (B/s) } \\ \omega_{s} & \text { Streamwise vorticity } \\ \psi & \text { Work coefficient } \\ \phi & \text { Flow coefficient } \\ \Omega_{\text {total }} & \text { Mass-averaged total pressure loss coefficient } \\ \Omega_{\text {secondary }} & \text { Mass-averaged dynamic pressure loss coefficient } \\ \Omega_{\text {profile }} & \text { Mass-averaged profile pressure loss coefficient } \\ & \end{array}$

\section{Superscripts and Overbars}

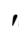

$\overline{\bar{\Omega}}$

\section{Subscripts}

1,2

a

CL

loc

W
Pitchwise mass-averaged flow quantity

Overall mass-averaged flow quantity

Area-averaged flow quantity

Upstream and downstream

Axial component of velocity

Centre line of blade span, $\mathrm{z} / \mathrm{h}=0.5$

Local

"whirl” (pitchwise or tangential) component of velocity 
WT Wind tunnel coordinate system

Abbreviations

3HP Three-hole probe

7HP Seven-hole probe

AC Alternating Current

ADC Analog-to-Digital

CEGV Compressor Exit Guide Vane

CFD Computational Fluid Dynamics

DAQ Data Acquisition

FSTI Freestream Turbulence Intensity

GUI Graphical User Interface

HP High Pressure (compressor or turbine)

LSTL Low-Speed Turbomachinery Laboratory

LSWT Low-Speed Wind Tunnel

LP Low Pressure (compressor or turbine)

PWA Pratt \& Whitney Aircraft

SKE Secondary Kinetic Energy 


\section{Chapter 1}

\section{Introduction}

\subsection{Research Motivation}

With thousands of aircraft flying every day, air travel has become one of the main sources of transportation and aircraft manufacturers are always trying to increase their presence in this market. The large market pushes them to develop aircraft that provide better performance while reducing fuel consumption, noise and emission levels while remaining cost-effective.

Most of these improvements come from the development of better engines. The next generation of Boeing and Airbus aircraft, as well as the currently in-development CSeries from Bombardier, are designed with new engine technologies that are intended to provide these improvements. The CSeries engine, the Pratt \& Whitney PurePower PW1500G shown on Figure 1.1, is an example of such a new engine.

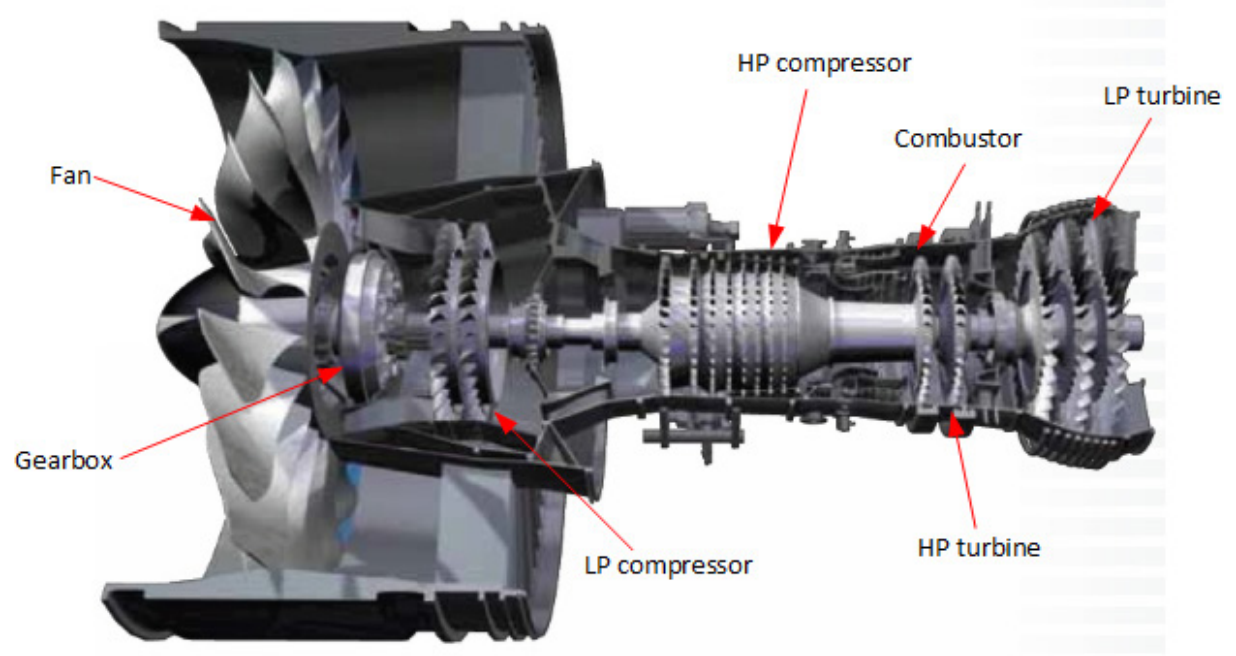

Figure 1.1 PW1500G gas turbine engine cut-away [Source: www.pw.utc.com] 
The engine manufacturers are continuing to work on more efficient and cost-effective engines. New aircraft engines must be fuel efficient and light weight, while also having low manufacturing and operating costs. Engine weight reduction and improved efficiency can be obtained from the optimization of the individual components. Typical engine components, shown on Figure 1.1, include the fan, the low-pressure (LP) and high-pressure (HP) compressors, the combustor and the LP and HP turbines.

Engine component efficiencies are already very high and close to the design limits. Engine manufacturer are looking into other alternatives to improve their designs to remain competitive in the market. For this reason, a lot of effort is currently being given towards the design of highly-loaded blades, which involves reducing the number of blades per row, and therefore reducing the weight of the engine. The highly-loaded blades offer similar performance with significant weight reduction, which directly results in cost savings. However, reducing the number of blades effectively results in increasing the aerodynamic loading of each blade. This has been shown to result in greater secondary flows, the threedimensional flows arising in a region near the endwalls, which are a significant source of losses (Zoric, 2006). Therefore, engine manufacturers have been looking at ways to reduce these losses and one of the potential improvements that have been examined is the application of contouring on endwalls of the blade rows. This consists on modifying the blade-passage endwall geometry to reduce the pressure gradients that drive the cross-flow at the endwall. This technology has been shown to alter the flow physics in a beneficial manner (Knezevici, 2011; Hartland et al., 2000; Harvey and Offord, 2008).

Additionally, an aircraft engine is operated at a variety of conditions such as idle, takeoff and cruise. In many instances, it is working at off-design values of blade incidence, which can result in an increase of the losses and in a reduction of the efficiency. It is then important to investigate the effects of working at off-design to understand how the flow field is affected and whether the endwall contouring remains effective. It is important to verify if these improvements also show benefits at these off-design conditions.

The motivation for the present work was to investigate the effect of the application of non-asymmetric endwall contouring on the flow field across a compressor linear cascade and 
how it affects the generation of total and secondary losses at design and off-design values of incidence.

\subsection{Research Objectives}

Secondary losses in turbine and compressor blade rows have been the topic of many investigations over the past decades. Means of secondary losses reduction via the application of endwall contouring have been the subject of several investigations, such as the ones from Hoeger et al. (2002), Harvey (2008), Reising and Schiffer (2009a), Lepot et al. (2011) and Hergt et al. (2011) on compressor rows, and the ones from Praisner et al. (2007), Knezevici (2011) and Taremi and Sjolander (2011) on turbine rows.

The objective of this thesis is to investigate the effect of non-axisymmetric endwall contouring on the secondary flows in axial compressors, and therefore on the loss generation across and downstream of compressor blade rows at design incidence $\left(0^{\circ}\right)$ and at off-design incidence $\left(+7^{\circ}\right)$. The blade row and the endwall profile were designed and built by Pratt \& Whitney Aircraft (PWA). The airfoil is denoted as CEGV-1 and represents a compressor exit guide vane row of an aircraft engine.

The thesis investigates the effect of the contoured endwall on the secondary losses generated across this highly-loaded compressor blade row. The results are compared with the losses for the baseline flat endwall in order to evaluate the potential benefits. In addition, the

effect of the application of endwall contouring is examined at off-design incidence to see if the potential benefit of contouring is also observed when the aircraft engine is operating at off-design conditions. All measurements were performed in a low-speed linear cascade at a constant Reynolds number of 150,000 based on axial chord and inlet centerline velocity.

\subsection{Overview of Thesis}

Chapter 2 reviews the literature most relevant to the present investigation. The physics of the endwall flow is described and the relationship between loss generation and various aerodynamic and geometric parameters is discussed. 
Chapter 3 provides an overview of the experimental apparatus, measurement procedures and data reduction methods. The low-speed wind tunnel and linear cascade test section are described first, followed by a description of the research cascade and of the endwall contouring used in this experiment. Next, the experimental instrumentation, the probe calibration apparatus and a description of the different probes used are presented. Uncertainty estimates are provided for the experimental instrumentation and for the pressure probes. Finally, the cascade testing procedures and data reduction methods are described.

The results of the secondary flow measurements at design and off-design incidences are presented in Chapter 4 and Chapter 5, respectively. These chapters examine the flow field through the blade passage with a flat endwall and the results are compared to the case where endwall contouring is applied. These chapters provide an experimental assessment of the performance of non-axisymmetric endwall contouring at both design incidence and $7^{\circ}$ of incidence.

Finally, Chapter 6 summarizes the conclusions from the present study and provides recommendations for future work. 


\section{Chapter 2}

\section{Background and Literature Review}

\subsection{Introduction}

This chapter gives an overview of the literature related to past investigations on secondary flows in axial flow compressors with an emphasis on the relationship between loss generation and various aerodynamic and geometric changes. The chapter starts with an overview of the flow field and losses in axial turbomachinery, followed by a detailed description of the losses in a linear cascade. The effects of significant aerodynamic and geometric parameters on the secondary losses are discussed next. The chapter concludes with a review of endwall contouring design techniques. This chapter provides a general overview of the open literature. Relevant references are discussed in greater detail in subsequent chapters.

\subsection{The Flow Field and Losses in Axial Turbomachinery}

The main features of a compressor passage flow field are shown in Figure 2.1 (from Sjolander, 2012) and will each be discussed in terms of their associated pressure losses. The conventional loss breakdown scheme consists of total-pressure losses being divided into individual components that are superimposed linearly. In general, empirical correlations are then used in the early stages of design to predict the pressure losses generated in each of the individual components. Examples of such correlations are given by Lieblein et al. (1953), Koch and Smith (1976) and more recently, by Konig et al. (1996) and Aungier (2003). The main loss components are: profile losses, tip-leakage losses and secondary losses. 


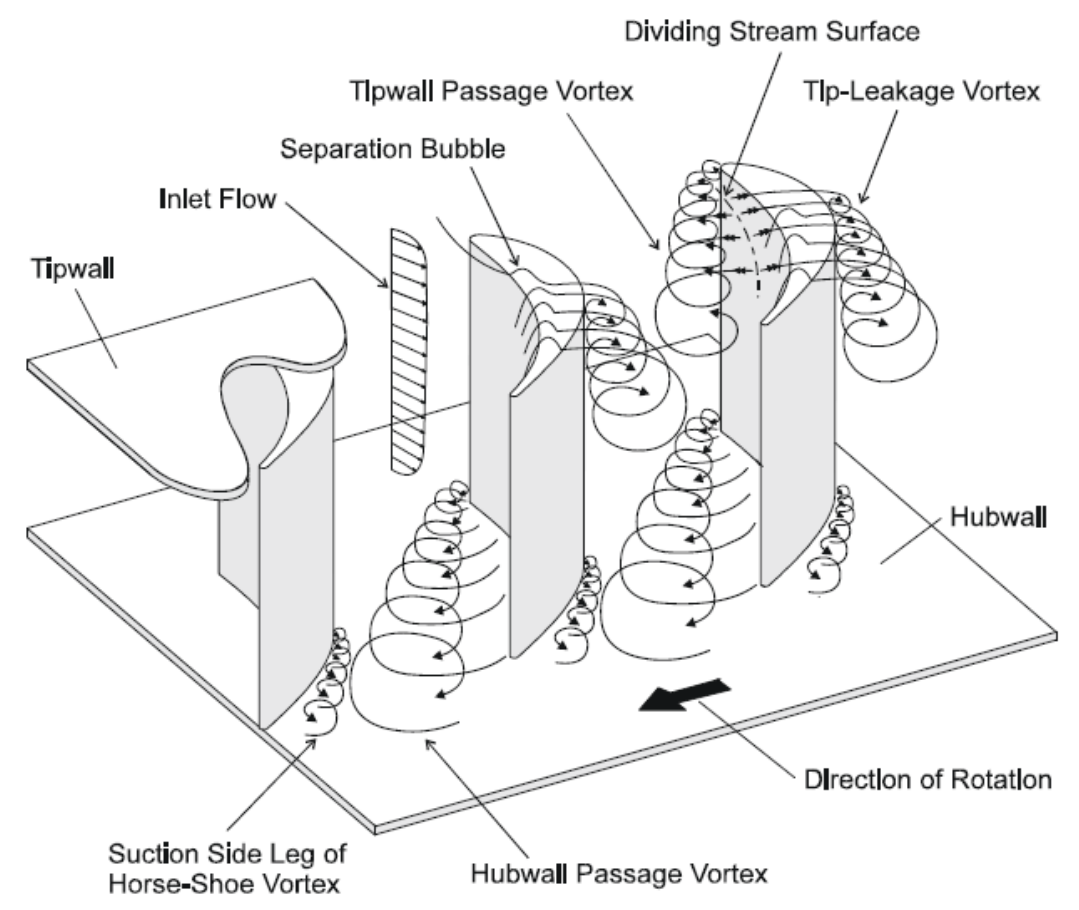

Figure 2.1: Flow through the blade passage of a compressor rotor (from Sjolander, 2012)

Profile losses are generated by the friction in the blade-surface boundary layer well away from the endwalls in a uniform two-dimensional flow. Losses generated by the sudden expansion in area at the trailing edge and by the mixing-out of the wake downstream of the blade are also generally included in the profile losses. The profile losses will be further discussed in Section 2.4.1.

Secondary losses are associated with the endwall boundary layers and the mixing of the various endwall vortical structures downstream of the blade row. The vortical structures, shown on Figure 2.1, are created from the interactions of the inlet boundary layer and the blade surface pressure distribution. Secondary losses are generated through high shear stresses at the endwall and through viscous mixing of the vortices with the downstream flow. The secondary loss structures are considered as a three-dimensional loss component because of the significant spanwise and pitchwise flow components of velocity in these regions. The secondary losses will be further discussed in Section 2.4.2.

Tip-leakage losses are generated through downstream mixing of the tip-leakage vortex with the surrounding fluid as shown on Figure 2.1. This vortex is formed from the flow 
rolling over the tip of the moving rotor blade. Viscous losses inside the gap are also included in the tip-leakage losses. Tip-leakage loss structures are also considered as three-dimensional because of the significant spanwise and pitchwise flow components of velocity in the tip region. The tip-leakage losses are not investigated in this study.

The current study will investigate the differences that arise in the secondary flows when a non-axisymmetric endwall contouring is used instead of a conventional axisymmetric endwall at design and off-design values of incidence.

\subsection{Blade Loading}

The blade loading is defined as the net tangential force acting on the airfoil crosssection. It is obtained by integrating the difference in static pressure between the pressure and suction surfaces of the blade over the axial chord distance. An example of velocity distribution (similar to static pressure distribution) around a compressor blade is shown on Figure 2.2.

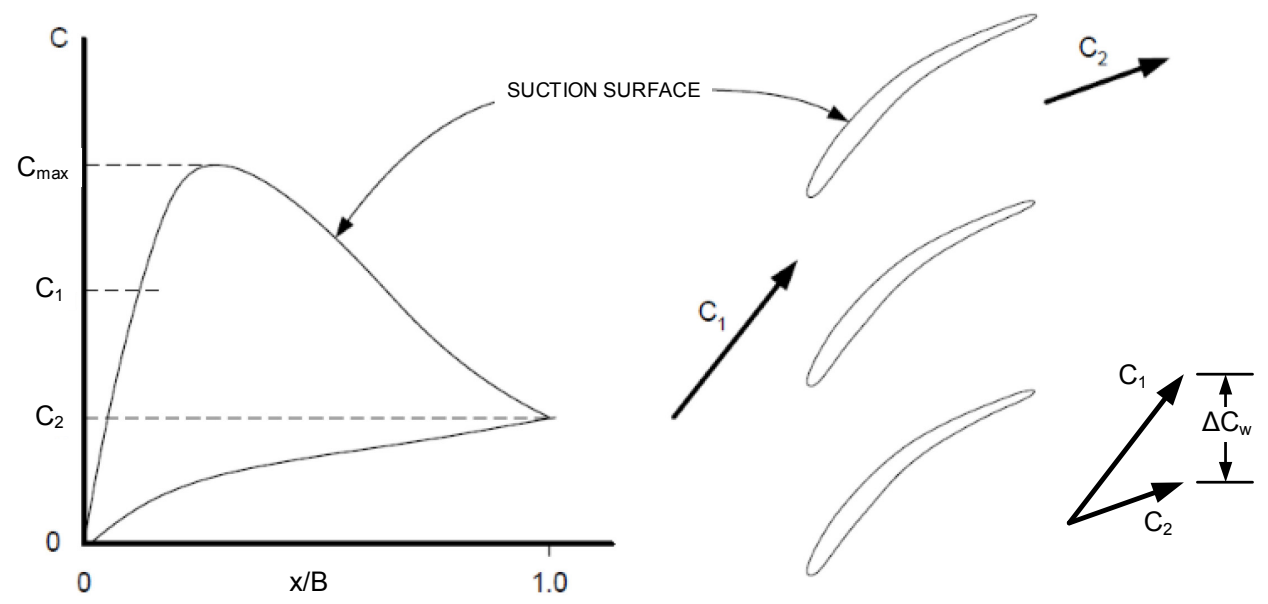

Figure 2.2: Compressor velocity distribution (adapted from Sjolander, 2012)

In the design process of an airfoil, the choice of solidity is important to ensure that the blade loading is within the limits that it can take. Solidity is defined as the ratio of the blade chord length over the spacing $(\sigma=\mathrm{B} / \mathrm{s})$. Following extensive series of cascade measurements 
on compressor airfoils, Howell (1945) presented a correlation that could be used to select the solidity based on the air outlet angle and air deflection. The selected solidity would result in the blade row operating at $80 \%$ of the stalling turning angle, which was found to be where the losses were close to a minimum (Sjolander, 2012).

In the 1950s, NACA also conducted an extensive set of cascade measurements to determine the performance of compressor airfoils. The performance of the blade is limited by the diffusion on its suction surface. If the diffusion is too large, the boundary layer separates from the blade surface, resulting in stalling the blade and increasing the losses significantly. From the results obtained by NACA, Lieblein et al (1953) developed a parameter that could be used to measure the severity of the diffusion. This parameter is known as the diffusion factor (D):

$$
D=1-\frac{C_{2}}{C_{1}}+\frac{\Delta C_{w}}{2 \sigma C_{1}}
$$

where $\sigma$ is the solidity of the blade, $\mathrm{C}_{1}$ and $\mathrm{C}_{2}$ are the inlet and outlet absolute velocities and $\Delta \mathrm{C}_{\mathrm{w}}$ is the difference in tangential velocities between the inlet and outlet flow. The velocity parameters can be seen on Figure 2.2. The diffusion factor is a common parameter used for the design of compressors and is still widely used. With the diffusion factor selected and the velocity triangles defined, the solidity can be calculated from Equation (2.1).

The diffusion factor has been found to correlate well with the profile losses. Figure 2.3, reproduced from NASA SP-36, shows that the total loss coefficient, $\omega_{1}$, only increase gradually with diffusion factor for values of less than approximately 0.6 (Bullock and Johnsen, 1965). However, from Figure 2.3, it can be observed that losses rise sharply as the diffusion factor increases beyond 0.6 . This characteristic implies boundary layer separation and onset of stall.

With the objective of reducing engine weight and cost, highly-loaded compressor blades with high diffusion factor and low solidity have been investigated by many researchers. Recent research shows diffusion factors in the range of 0.40-0.60 (Hoeger et al., 2002; Ellison, 2005; Harvey, 2008; Sonoda et al., 2008; Dickens and Day, 2009, Reising and Schiffer, 2009a, Lepot et al., 2011). For comparison purposes, the current study is done on a compressor exit guide vane with a diffusion factor of 0.52 . 


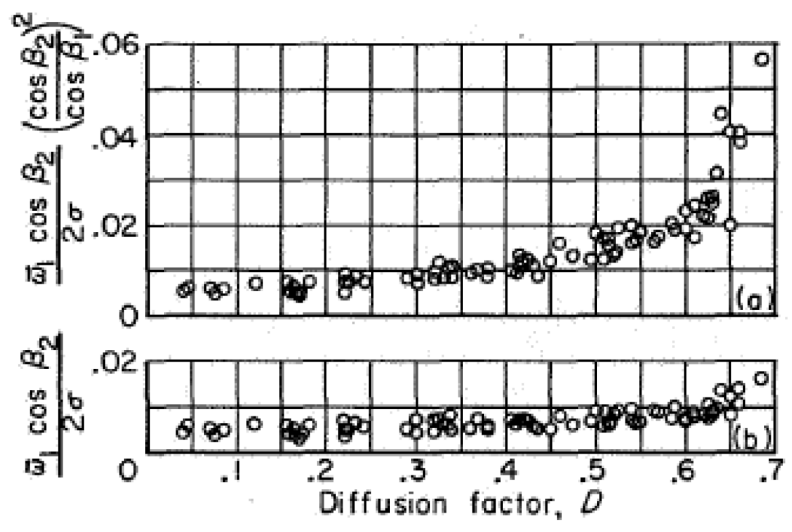

(a) Based on $\bar{\omega}_{1}\left(\frac{\cos \beta_{2}}{\cos \beta_{1}}\right)^{2}$.

(b) Based on $\bar{\omega}_{1}$.

Figure 2.3: Variation of compressor total pressure loss coefficient with diffusion factor, D (Bullock and Johnsen, 1965)

\subsection{Profile and Secondary Losses in Linear Compressor Cascades}

\subsubsection{Profile Losses}

The profile pressure losses described in Section 2.2 are generated within the twodimensional blade surface boundary layers well away from the endwalls. The entropy created in a boundary layer is due to the viscous shear caused by the velocity gradients. Most of the entropy generation in boundary layers is concentrated in the inner part of the boundary layer where the velocity changes most rapidly and where the shear is highest (Denton, 1993). In a linear cascade such as the one used in the present study, it is assumed that the measured midspan losses are representative of the profile losses, although the midspan flow is never precisely two-dimensional (Benner, 2003). The profile losses are strongly dependent on the state of the pressure and suction surface boundary layers, on the streamwise pressure gradient and on the Reynolds number.

In low Reynolds number applications, the boundary layers could be laminar. Denton (1993) shows that the losses increase rapidly at very low Reynolds number because of high dissipation in the boundary layers. In addition, he shows that laminar separation of the boundary layer may also occur if the adverse pressure gradient on the suction surface is large 
enough. The flow may be fully stalled or may reattach and form a separation bubble. In the cases where flow separation is unavoidable, the latter would be preferred as the losses generated are much lower than for the fully-stalled condition.

Boundary layer separation is a dominant concern in the design of compressor blades (Denton, 1993). Because of the adverse pressure gradient present on the suction surface of a compressor blade, it is possible that separation will occur, resulting in an increase in losses. Thus, it is important to be able to predict boundary layer transition, separation and reattachment on compressor blades in order to mitigate the losses.

Additionally, Denton (1993) states that the local boundary layer loss generation is proportional to the cube of the local freestream velocity. Therefore, the suction surface is dominant in producing loss since that is where the surface velocity is highest (Denton, 1993).

Other important contributors to the profile losses are the losses generated at the trailing edge of the blade. The high rates of shearing that occur in the wakes behind the trailing edge will generate entropy. For compressor blades, the trailing edge blockage is usually small but, since the boundary layers are thicker, the boundary layer displacement thickness and trailing edge thickness might contribute more to the loss coefficient. The viscous dissipation in the wake is a direct result of the boundary layers on the blade surfaces. Denton (1993) suggests that approximately $1 / 3$ of the stagnation pressure loss are generated behind the trailing edge of the blade.

\subsubsection{Secondary Losses}

A detailed interpretation of the flow field is necessary to further understand the mechanisms of the secondary loss generation. A large amount of work has been done on secondary flows in axial turbomachinery for both turbines and compressors. Many previous studies of secondary losses in axial compressors have resulted in a good physical understanding of the flow behaviour: Adkins and Smith (1982), Gallimore and Cumpsty (1986), Wisler et al. (1987), Kang and Hirsch (1991), Cumpsty (2004), Gbadebo et al. (2004) and Lei et al. (2008).

The secondary losses are generated by the viscous shearing that occurs between vortices and also, between vortices and the freestream fluid as they mix downstream of the 
blade row. Additionally, secondary losses are also generated within the endwall boundary layers. The general interpretation of the three-dimensional flow structures by Harvey (2008) is described below and is based on the turbine secondary flow model of Takeishi et al. (1990) shown on Figure 2.4. The model shown on Figure 2.4 is also valid for compressors since the main features of the secondary flow field are largely the same in axial compressors and turbines.

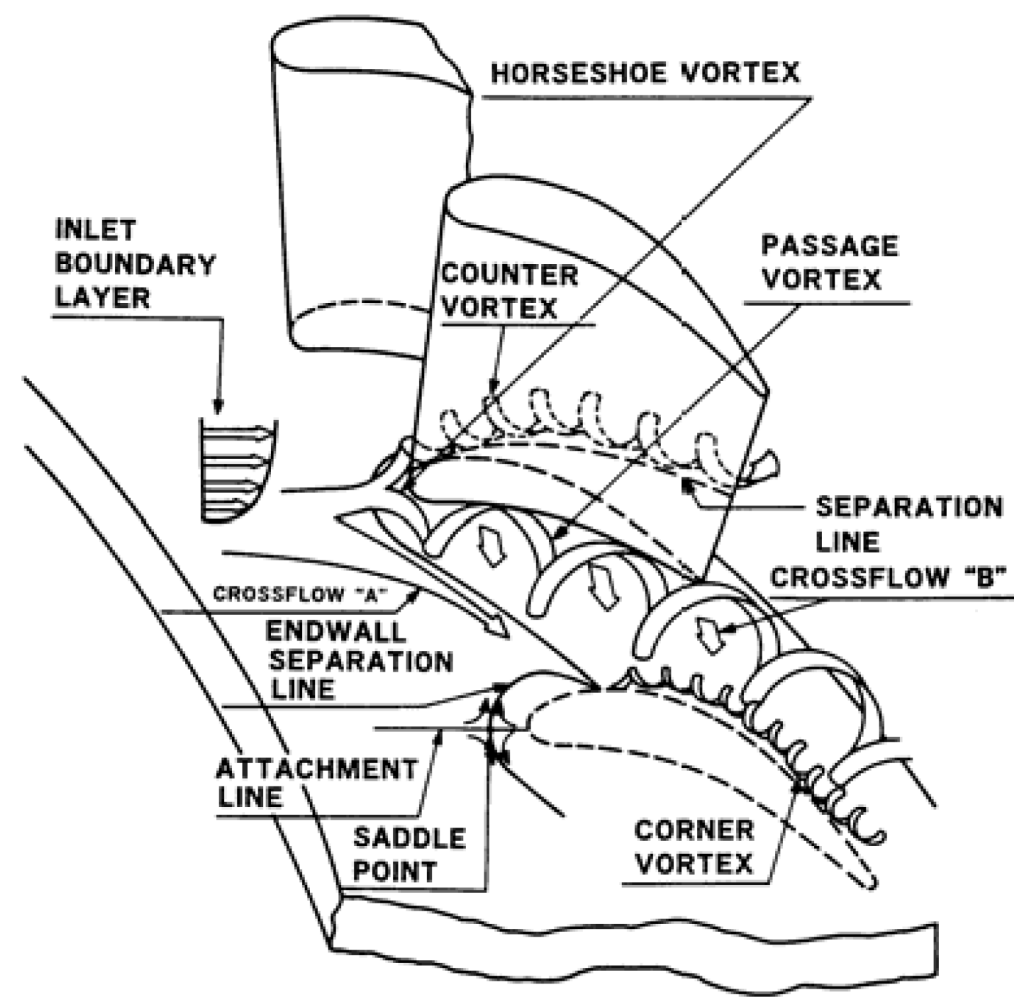

Figure 2.4: Interpretation of endwall flow features (from Harvey, 2008)

The dominant components of the secondary flow in a compressor passage are the horseshoe vortex, the passage vortex, the counter vortex, the corner vortex and the trailing edge vortices (not shown). As shown on Figure 2.4, the inlet boundary layer flow near the blade leading edge rolls up and separates at a location known as the saddle point. The separation is induced by the adverse streamwise pressure gradient due to the blade stagnation point and gives rise to the horseshoe vortex. As shown, the legs of the horseshoe vortex develop into a complex and highly three-dimensional flow. 
The pressure side leg of the horseshoe vortex becomes the core of the passage vortex, which is the dominant part of the secondary flow. The passage vortex is largely responsible for the size and strength of other vortical structures present in the secondary flow. The low momentum fluid in the endwall boundary layer is "over turned" by the blade-to-blade (crosspassage) pressure gradient. The fluid is swept towards the suction side of the passage until it encounters the blade surface, at which point the fluid is forced up the blade surface and rolls up into the passage vortex. Under the passage vortex, a new endwall boundary layer forms at the pressure side endwall corner (cross-flow "B'). This low momentum fluid is entrain by the pressure leg of the horseshoe vortex and ramps up on the suction surface of the blade, at which point there is a strong interaction with the suction surface boundary layer (Benner et al., 2004). The fluid ramps up on the suction surface until it separates along the "blade separation line" shown on Figure 2.4.

The suction side leg of the horseshoe vortex rotates in the opposite direction as the passage vortex. This leg of the horseshoe vortex often moves away from the endwall and remains above the passage vortex while the latter grows as it moves downstream.

Another type of vortex can also form at the suction surface endwall corner: the corner vortex. This vortex, if present, rotates in the opposite direction as the passage vortex, such that it will result in reducing the overturning of the flow near the endwall. The formation of this vortex will generate additional losses.

Although the basics structures of the secondary flows are similar for turbines and compressors, there are some important differences that are summarized by Harvey (2008). Typically, the turning in a compressor blade row is much less than in a turbine one. Also, the boundary layer is much thicker, up to half the blade chord for multistage compressors, and the boundary layers are being decelerated (Denton, 1993). Therefore, because the fluid decelerates through the blade passage, it will be more affected by the cross-passage pressure gradient. This low momentum but high entropy fluid will accumulate in the suction surface endwall corner and further decelerate due to the overall pressure increase across the row. This will often lead to reverse flow on the endwall and form a large corner separation. This is known as corner stall. The formation of the corner stall, illustrated by Lei et al. (2008), is shown on Figure 2.5. 


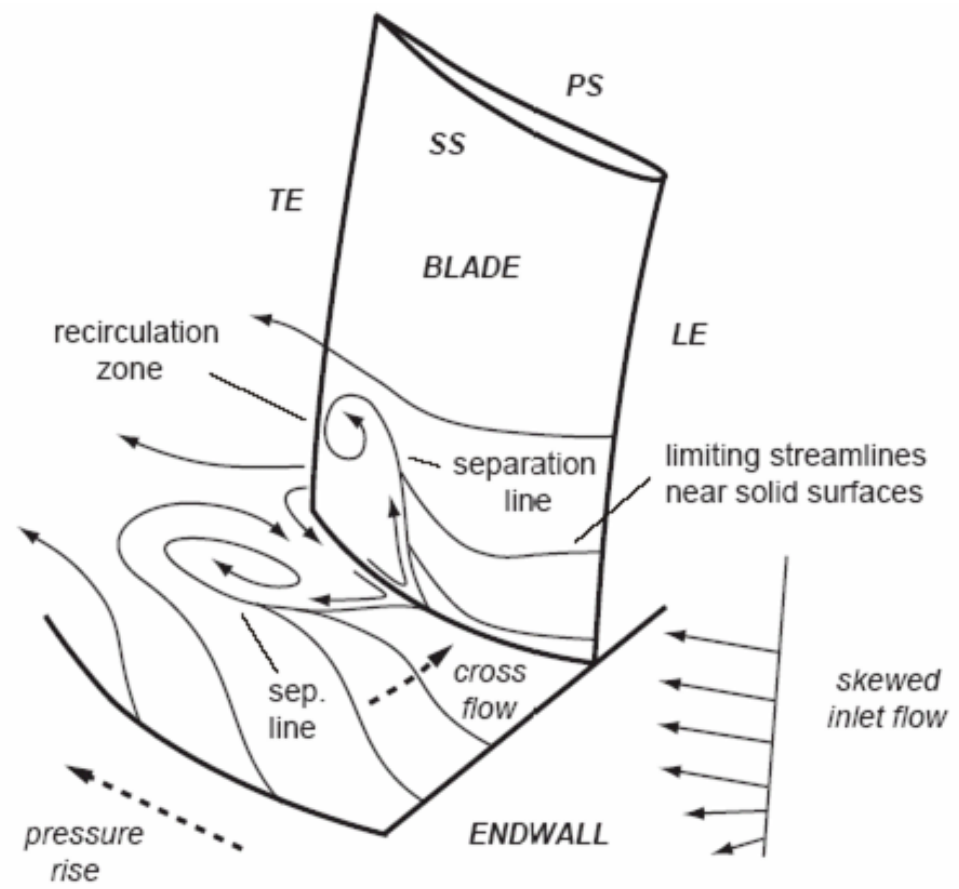

Figure 2.5: Formation of hub corner stall, from Lei et al. (2008)

\subsection{Research on Secondary Losses in Linear Cascades}

\subsubsection{Introduction}

The study of secondary losses is very important for the improvement of compressor design. Secondary losses in a compressor cascade can account for a large percentage of the total loss across the blade row. Howell (1945) quoted a value of $2 / 3$ of the loss due to annulus and secondary losses at design conditions. Lakshminarayana (1996) noted, from his high aspect ratio blade in a compressor cascade, a value of approximately $35 \%$ of the total losses.

The following sections will discussed some of the aerodynamic variables known to affect the secondary loss generation. 


\subsubsection{Effect of Inlet Boundary Layers and Freestream Turbulence Intensity}

The inlet boundary layer was not varied in the present study, but the choice of an appropriate boundary layer is necessary to have meaningful secondary flow results. The current boundary layer is representative of previous studies conducted in the Low-Speed Wind Tunnel (LSWT) at Carleton University. In the present study, the inlet boundary layer is fully turbulent with a shape factor $\left(\mathrm{H}_{\mathrm{SF}}\right)$ of 1.2-1.3 and a thickness of approximately $12-13 \%$ of the blade span. The shape factor in the current study is similar to the values obtained by Knezevici et al. (2008) and MacIsaac et al. (2010) in their turbine cascade studies performed in the same wind tunnel.

Choi et al. (2008) conducted numerical investigations of the effect of inlet boundary layer thickness on the rotating stall using a low-speed axial compressor rotor blade (NACA 65 profile) at a constant Reynolds number of 244,000 (based on axial chord and inlet velocity at design condition). The investigations showed that, at design condition, the inlet boundary layer thickness had minimal effect on the size of the hub-corner separation. However, as the loading was increased up to the near-stall condition, it was found that the size of the hubcorner separation increased significantly for the thick boundary layer, whereas the size remained fairly insensitive to the loading for the thin boundary layer. Therefore, it was found that increasing the loading on a thicker boundary layer led to significant loss in performance across the blade row, but it had little effect on the performance with the thin boundary layer.

The Freestream Turbulence Intensity (FSTI) was not varied in the present experiment, but it is mentioned here since it is important to the mechanisms of loss generation. The FSTI affects the state of the blade boundary layer. At low Reynolds number and low FSTI, the adverse pressure gradient is sufficient to cause a laminar separation of the boundary layer downstream of the suction peak, followed by a turbulent flow reattachment. A higher FSTI could accelerate the transition process from a laminar to a turbulent flow. This would allow the transition to occur on a shorter distance and could result in a smaller separation bubble. The smaller bubble would generate less entropy, thus reducing the profile losses. The FSTI also affects the secondary losses through the interaction of the secondary flows with the suction surface separation (i.e. bubble). The shorter separation bubble due to higher FSTI 
would reduce its interaction with the secondary flows, therefore resulting in less loss being generated.

Many researchers investigated the effect of FSTI in compressor cascades. Herrig et al. (1957) and Blight and Howard (1952) investigated the effect of Reynolds number and FSTI on the total pressure loss coefficient, $\omega$, in two-dimensional compressor blade cascades. The studies by Herrig and Blight were performed on NACA-65 and Lighthill blades, respectively. Both studies showed that, for a constant Reynolds number, the total pressure loss coefficient reduced as the turbulence intensity increased as it can be observed on Figure 128 of NASA SP-36 (Bullock and Johnsen, 1965).

Köller et al. (2000) and Küsters et al. (1999) performed design and optimization studies on a compressor blade and the results showed that, for high turbulence intensity, boundary layer transition on the blade suction surface moved closer to the leading edge of the blade, ahead of the suction peak (i.e. point of maximum velocity), as the Reynolds number was increased. Similar conclusions were obtained from the analytical studies performed on a compressor cascade by Schreiber et al. (2002). The blade profile used had a design inlet Mach number of 0.6 , a flow turning of $16^{\circ}$ and a suction peak at $30 \%$ chord. Using the bladeto-blade solver MISES (from Drela and Youngren, 1991), Schreiber performed multiple simulations to show the influence of Reynolds number and FSTI on the laminar-to-turbulent flow transition onset and the results are shown on Figure 2.6. As observed, the transition onset was found to be significantly dependent on the Reynolds number and on the FSTI. At high Reynolds number (above $0.5 \times 10^{6}$ ), transition moved closer to the leading edge of the blade as the turbulence intensity was increased up to a point where the transition would occur in the accelerated region of the blade. However, for low Reynolds number (less than $0.2 \mathrm{x}$ $10^{6}$ ), the transition onset was found to only move gradually towards the leading edge of the blade, but always remained downstream of the suction peak. 


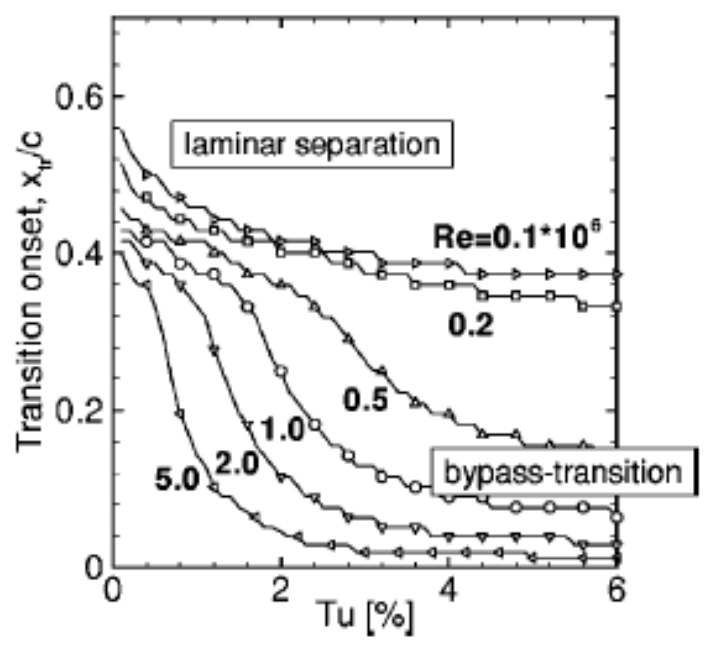

Figure 2.6: Influence of Reynolds number and FSTI on suction surface transition onset (from Schreiber et al., 2002)

Douglas et al. (2003) investigated the effect of FSTI on the flow field of a compressor cascade with high camber of $55^{\circ}$ over a large range of inlet flow incidences and for Mach numbers varying from 0.55 to 0.89 . The experiment was conducted with and without turbulence generating grids, resulting in FSTI of $1.6 \%$ and $0.4 \%$, respectively. It was found that, for Mach numbers of less than 0.75, the turbulence intensity had minimal effect on the losses at negative inlet flow incidences. However, over the range of Mach numbers studied, it was found that increasing the turbulence intensity significantly decreased the total losses across the blade row at design and positive incidences when compared to the low turbulence intensity case. Also, the higher turbulence intensity increased the incidence at which the suction surface boundary layer separated, therefore increasing the effective operating range of the cascade (i.e. the range of inlet flow incidences where the loss coefficient is less than twice the minimum loss level).

Muthanna (2002) investigated the effect of FSTI on the flow field through a compressor cascade with tip leakage. The grid used generated a turbulence intensity of approximately $3 \%$. Muthanna found that the higher turbulence intensity resulted in reductions of $4 \%$ in the blade loading and of $20 \%$ in the vorticity levels in the tip leakage vortex. 


\subsubsection{Effect of Blade Loadings and Blade Loading Distributions}

The Smith chart shown on Figure 2.7 (from Dickens and Day, 2009) is a useful figure to show the influence of the flow coefficient and work coefficients on the stage efficiency. The flow coefficient $\left(\phi=C_{a} / U\right)$ corresponds to the ratio of axial absolute velocity per unit of blade circumferential speed. The work coefficient $\left(\psi=\Delta h_{0} / U^{2}\right)$ is defined as the change in enthalpy $\left(\Delta h_{0}=U \Delta C_{w}\right)$ per the square of the blade circumferential speed and it is used as a measure of the stage loading. For good efficiency, the design values should be selected within 0.2-0.7 for the flow coefficient and 0.3-0.6 for the work coefficient.

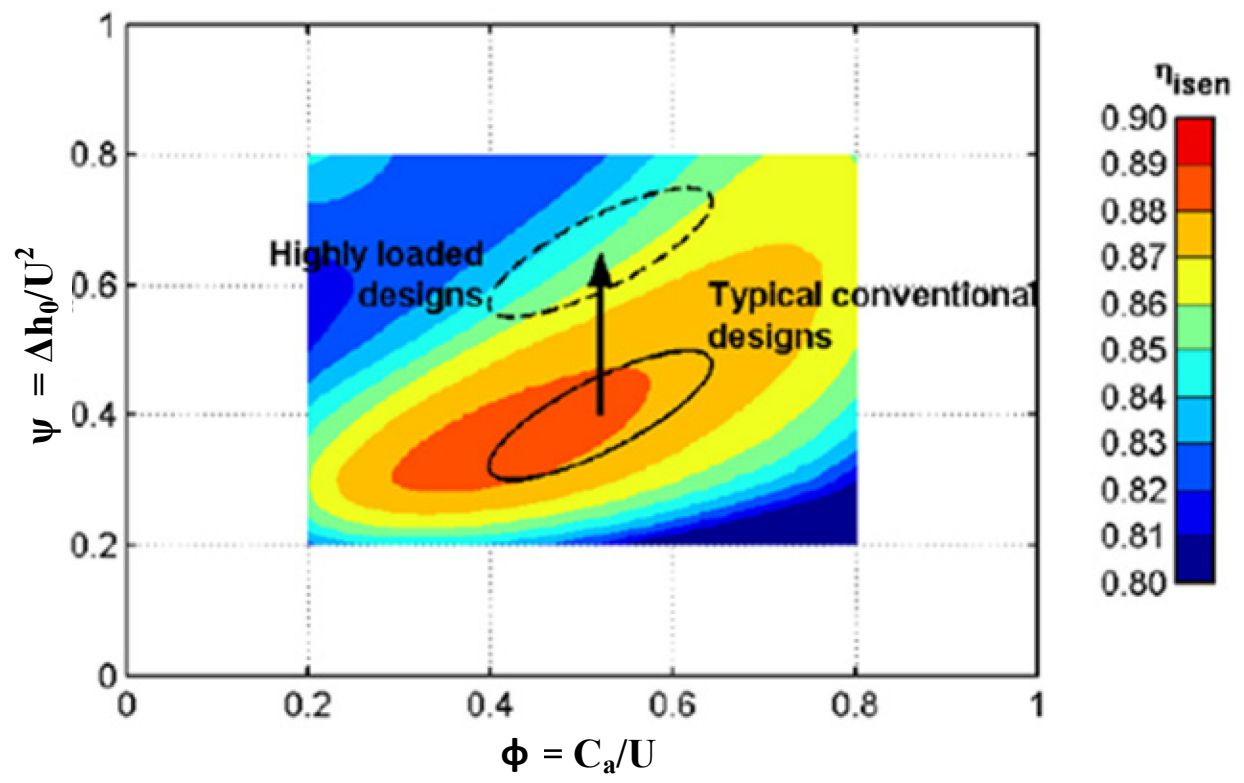

Figure 2.7: Compressor Smith chart with contours of efficiency as a function of work coefficient and flow coefficient (adapted from Dickens and Day, 2009)

Dickens and Day (2009) performed CFD investigations on four compressor stages of different blade loadings to investigate how the losses are affected by the loading. The investigations were done on three highly-loaded stages $(\psi=0.55,0.65$ and 0.75$)$ and on a more conventional loaded stage ( $\psi=0.45$ ) as shown on Figure 2.7. From their studies, it was shown that, for de Haller number $\left(\mathrm{C}_{2} / \mathrm{C}_{1}\right)$ above approximately 0.64 , the stator losses were primarily dependent on the blade loading. The de Haller number is a measure of the level of diffusion a compressor blade row can sustain and can be represented by the amount of 
turning the blade row needs to do. High values correspond to low flow turning, and therefore, low diffusion across the row. The studies showed that, at de Haller number above 0.64, the loss coefficient significantly increased with the blade loading. This region corresponds to the diffusion limit of the passage. However, Dickens and Day showed that a further increase in the flow turning (i.e. reduction in the de Haller number below 0.64) and in the blade loading resulted in the formation of large corner separations, which generated additional losses and reduced the efficiency of the blade row. Dickens and Day showed that, in order to prevent generating larger losses from the increased loading, it is necessary to off-load the stator by reducing its inlet flow angle, therefore, increasing the de Haller number. However, this results in an increased degree of reaction, meaning that more of the energy transfer is done by the rotor. In their study, it was shown that increasing the loading of the rotor also increased the losses, but to a smaller extent than for the stator and that losses were almost independent from the inlet flow angle of the rotor.

Sonoda et al. (2008) studied the endwall performance of two outlet guide vanes with different blade loading distributions at two different Reynolds numbers. The compressor blades used for the experiment had a diffusion factor of 0.53 , similar to the one in the current study $(\mathrm{D}=0.52)$. The more front loaded compressor blade showed improved twodimensional behaviour along the span, especially at the lower Reynold's number, where the wakes were significantly thinner. Additionally, it was found that the front loaded blade had stronger secondary flows due to the earlier onset of the cross-passage pressure gradient. Previous studies of the effect of blade loading distributions on turbine rows showed that front loaded blades produced higher secondary losses when compared to aft loaded blades (Popovic, 2005; Zoric, 2006). However, unlike in turbines, Sonoda et al. found that the front loaded blade generated lower secondary losses compared to the slightly more aft loaded one. The static pressure levels in the endwall corner were higher than at midspan, creating a spanwise pressure gradient. Sonoda et al. found that the stronger cross-passage pressure gradient from the more highly-loaded blade reduced the chordwise extent of the spanwise pressure gradient, therefore reducing the amount of low energy fluid moving up the blade into the region of three-dimensional separation. 


\subsubsection{Effect of Inlet Flow Incidence}

The inlet flow incidence has significant effect of the corner stall present in a compressor row. Thus, several investigators, such as Dickens and Day (2009), Ma et al. (2011), Gbadebo et al. (2004), Harvey (2008) and Kozulovic et al. (2009) investigated the effect of inlet flow incidence on loss generation on compressor linear cascades. The effect of inlet flow incidence on turbine linear cascades has been investigated by other researchers, such as Benner et al. (1997) and Zoric et al. (2007b). The above studies showed that positive inlet flow incidence resulted in an increase in both profile and secondary losses across the blade row for both compressor and turbine cascades.

A positive inlet incidence means that the blade row has to do more flow turning than at design incidence and, as a result, the blade loading increases. This is mainly due to the pressure distributions upstream of the suction peak. Ma et al. (2011) investigated the effect of incidence by performing measurements at five different values of incidence from $-2^{\circ}$ to $6^{\circ}$. It was found that the static pressure on the pressure surface of the blade increased with positive incidence, whereas the static pressure on the suction surface decreased up to the suction peak. These changes in static pressures at higher incidences resulted in an increase in blade loading and in higher velocity at the edge of the suction surface boundary layer. As shown by Denton (1993), entropy production in boundary layers varies with the cube of the boundary layer edge velocity. Therefore, as a consequence of the increased blade loading, the profile losses across the blade row increase with positive incidence.

In addition, Ma et al. (2011) found that the flow on the suction surface of the blade decelerated after the suction peak until the static pressure reached a constant plateau, corresponding to the flow separating from the blade surface. It was found that the extent of separation increased with incidence and that the position of initial separation moved closer to the leading edge of the blade as the incidence increased. As a consequence of the flow separation, the size of the wake downstream of the trailing edge also increased. As mentioned in Sections 2.2 and 2.4.1, the dissipation and mixing-out of the wake is an additional source of profile loss, such that the larger wakes at higher values of inlet flow incidence would result in larger profile losses. 
Gbadebo et al. (2004) numerically investigated the effect of incidence on the size of the three dimensional separations in a compressor cascade with five blades and with a diffusion factor at midspan of 0.49 . The investigations were done at four different values of incidence from $-7^{\circ}$ to $3^{\circ}$. Gbadebo et al. found that the size of the thee-dimensional separation on the suction surface of the blade was fairly insensitive to negative inlet flow incidence. However, it was found that the chordwise and spanwise extent of the threedimensional separation increased significantly with positive incidences, which would lead to higher losses as the separated flow mixes with the main passage flow.

Similar observations were made by Harvey (2008) and Kozulovic et al. (2009) in their investigations on compressor cascades. In both investigations, it was found that the increase in positive incidences led to the formation of a large corner stall, which resulted in higher losses and higher flow underturning in the endwall region.

\subsection{Control of Secondary Flows for Secondary Loss Mitigation}

\subsubsection{Introduction}

This section presents different flow control methods that have been developed and investigated for controlling secondary flows in axial compressors and reducing the losses associated with them. Sections 2.6.2 through 2.6.4 review three different methods that have shown some benefits in loss reduction: namely endwall fences, leading edge modifications and axisymmetric endwall contouring. However, these methods were not investigated in the current research. Section 2.6.5 focuses on the non-axisymmetric endwall contouring method, which is the flow control technique investigated in the present study.

\subsubsection{Endwall Fences}

Endwall fences affect the cross-passage flow by constraining and deflecting its migration across the passage with the goal of weakening the secondary flow or passage vortex and thus reducing the secondary losses.

Hergt et al. (2011) performed experimental and numerical investigations of the application of endwall fences on a linear compressor cascade. The endwall fences were used 
to deflect the endwall cross-flow downstream of the passage and to reduce its interaction with the blade suction surface boundary layer. The fences delayed the onset of corner separation and reduced the size of the passage vortex, which resulted in a reduction of the cascade losses. Hergt et al. tested multiple configurations of fence arrangement and found that the optimal endwall fence arrangement for their cascade was composed of six fences of variable heights installed parallel to the blade. The fences were installed such that the smallest one was inserted near the mid-passage with the subsequent ones increasing in size up to the last fence near the suction surface. The smallest and tallest fence heights corresponded to $1 \%$ and $10 \%$ of the blade span, respectively. This fence arrangement led to a reduction in the corner stall and a decrease of approximately $9 \%$ in the cascade losses at design conditions.

However, since fences are costly to manufacture and there are questions about their durability, particularly in turbines, they have not been used in any engine applications.

\subsubsection{Leading Edge Modifications}

Endwall boundary layer control in a linear cascade can also be done by modifying the profile of the blade leading edge at the endwall. Modifications such as leading edge fillets are intended to have similar effects as the fairing between the wing and the fuselage of an aircraft, namely to weaken the horseshoe vortex formed at the wing-fuselage junction. The spanwise pressure gradient created by the modifications to the leading edge of the blade near the endwall is intended to reduce the secondary losses.

Müller et al. (2004) tested five different endwall configurations of a compressor blade leading edge in a low-speed cascade wind tunnel and compared the losses generated from each configurations to the losses generated from their baseline cascade with no leading edge modification. The five leading edge modifications investigated were: a bulb, medium fillet, large fillet, medium fillet with a blunt leading edge and large fillet with a blunt leading edge. The blunt leading edge is made by milling the fillet of the blade in the axial upstream direction in order to maintain the axial length of the blade (Müller et al., 2004). It was found that all leading edge geometries modified the endwall pressures along the passage. For the bulb configuration, the suction side of the horseshoe vortex counter-rotated relative to the passage vortex and was stronger than the baseline configuration. The stronger vortex 
weakened the interaction between the suction surface boundary layer and the passage vortex and also reduced the flow underturning at the highest loss spanwise location. This contributed to a reduction of approximately $17 \%$ and $28 \%$ in the secondary losses at $0^{\circ}$ and $3^{\circ}$ of incidence, respectively, when compared to the baseline configuration. For the fillet and blunt fillet configurations, a new vortex was observed: the fillet vortex. This vortex was found to rotate in the same direction as the passage vortex and to affect the interaction between the passage vortex and the suction surface boundary layer. The medium fillet, the medium blunt fillet and the large fillet configurations showed reduction in secondary losses of approximately $7-12 \%$ and $13-23 \%$ at $0^{\circ}$ and $3^{\circ}$ of inlet flow incidence, respectively. However, the large blunt-fillet showed a significant increase in secondary losses at design incidence $(\sim 24 \%)$ and a slight reduction at off-design incidence $(\sim 3 \%)$ due to a strong flow separation maintained throughout the whole passage.

Goodman and Miller (2010) also investigated the impact of modifications to the leading edge geometry of a compressor blade near the endwall. The different geometries tested included circular and elliptical leading edges and leading edge fillets. Following their investigations, it was found that the use of leading edge fillets slightly reduced the endwall losses compared to the no fillet test case. However, the losses were fairly insensitive to the size of the fillets. Also, it was found that, for their compressor blade with an elliptical leading edge, the secondary losses were approximately half the losses with the circular leading edge. For the elliptical leading, it was found that the boundary layer at the leading edge was laminar whereas it was turbulent for the circular leading edge, leading to higher endwall losses.

\subsubsection{Axisymmetric Endwall Contouring}

Endwall boundary layer control in a linear cascade can also be achieved by contouring the endwalls of the blade passage. Axisymmetric endwall contouring modifies the span of the blade passage by means of concave or convex streamline curvatures in the main flow direction and applied across the blade passage. The effect of streamline curvatures will be discussed in Sections 2.6.5 and 3.5. 
Hoeger et al. (2002) experimentally investigated the impact of axisymmetric endwall contouring on a transonic compressor rotor hub. The secondary flows generated from their concave contoured design were compared to a baseline flat endwall. It was found that the contoured endwall reduced the loading near the trailing edge of the blade and modified the shock pattern from an oblique shock to a normal shock. The normal shock resulting from the concave endwall was stronger than the oblique shock, but occurred closer to the leading edge of the blade and over a smaller region of the flow field. The flow from the flat endwall was nearly choked and drove the oblique shock over a larger chordwise distance. Following their experimental investigations, it was found that the application of the concave endwall resulted in a reduction in loss coefficient of approximately $30 \%$, which led them to conclude that moving away from near-choked flow conditions was more beneficial even if a stronger normal shock was generated.

Dorfner et al. (2007) presented a CFD investigation of a novel design of axisymmetric endwall contouring created from B-Spline curves and tensor product surfaces. The blade row used in this study is a Controlled Diffusion Airfoils and is representative of an outlet guide vane. The optimization process for the axisymmetric endwall profile included a total of 30 free variables that could be adjusted in the spanwise direction within the blade passage to alter the secondary flows. The design tool created by Dorfner showed that it could be used to strongly influence the flow field near the endwall.

Kröger et al. (2011) conducted a numerical study of the application of axisymmetric endwall contouring on an industrial gas turbine compressor. The study was divided into three parts which included an analytical approach to understand and predict the effects of contouring, an industrial application of axisymmetric casing contouring and a full CFD simulation of the rear stages of the SGT5-8000H Siemens gas turbine compressor prototype. The optimization of the axisymmetric casing contouring was performed on a typical high pressure compressor rotor at design conditions. This design demonstrated a small improvement in the stage efficiency of approximately $0.8 \%$ and the CFD simulations of the Siemens compressor model with an optimized casing contoured endwall showed an improvement in overall isentropic efficiency of $0.3 \%$. Kröger et al. concluded that the use of axisymmetric endwall contouring could provide benefits. 


\subsubsection{Non-Axisymmetric Endwall Contouring}

Non-axisymmetric endwall contouring is used as another approach to control the endwall flow. This approach was originally developed on axial flow turbines and its effect on endwall flow and on the blade row losses has been the subject of several investigations, such as the ones from Rose (1994), Harvey et al. (2000), Hartland et al. (2000) and more recently, Praisner et al. (2007) and Knezevici et al. (2008). The application of non-axisymmetric endwall contouring as a means of endwall flow control on compressor blade rows has only been the subject of recent studies, with the work of Harvey (2008), Reising and Schiffer (2009a, 2009b), Lu et al. (2009) and Lepot et al. (2011).

Non-axisymmetric endwall contouring consists on modifying the local static pressure by means of streamline curvature. It uses a combination of concave and convex wall curvatures with the objective of altering the blade cross-passage pressure gradient. Convex curvatures locally accelerate the flow and therefore reduce the local static pressure, whereas concave curvatures are used to diffuse the flow and locally increase the static pressure. The overall objective of non-axisymmetric endwall contouring in turbine or in compressor blade rows is to control the secondary flows and reduce the losses associated with them. However, the means to achieve these objectives can be different between turbines and compressors.

In turbine blade rows, non-axisymmetric endwall contouring is used to modify the cross-passage pressure gradient in order to limit the migration of the pressure-side leg of the horseshoe vortex and endwall boundary layer fluid towards the suction surface of the adjacent blade. Limiting the migration of the vortex and endwall boundary layer fluid results in the formation of weaker secondary vortices and in a reduction of the secondary kinetic energy (SKE) associated with them. The dissipation of SKE generates additional losses through mixing as the flow moves downstream. Therefore, the reduction in SKE becomes the main benefit of endwall contouring for turbine blade rows as it results in a reduction of secondary losses. Additionally, endwall contouring can also be used to reduce the secondary flows angle variations to provide a more uniform flow at the inlet of the next blade row and to improve its performance.

The effective goal of non-axisymmetric endwall contouring in highly-loaded compressor blade rows differs from the one in turbine blade rows due to the differences in the 
flow physics. The objective is to increase the strength of the cross-passage pressure gradients in order to drive high momentum fluid into the suction corner, and thereby mitigate the corner stall and reduce the losses (Harvey, 2008). For highly-loaded compressor blade rows, the corner stall is a significant source of losses, thus its reduction becomes the main benefit of endwall contouring. The cross-passage pressure gradient is altered to enhance the crossflow on the endwall and the migration of the secondary flows towards the endwall/suction surface corner in order to delay and/or reduce the corner stall and therefore, reduce the overall losses. The main disadvantage of increasing the strength of the cross-passage flow is the increase in SKE, which will generate additional losses as it dissipates and mixes downstream. Also, the stronger cross-flow inside the blade passage can possibly generate higher viscous losses in the endwall boundary layer due to the higher wall shear stresses. However, for highly-loaded compressor rows, it is expected that the net benefit of increasing the cross-passage flow to mitigate the corner stall more than offsets the additional losses generated from the SKE, such that the result is a net reduction of overall losses. For more lightly loaded compressor rows, however, the corner stall might not be the dominant source of losses and its mitigation by increasing the cross-flow might not provide the same benefit as for highly-loaded blade rows. Thus, it is conceivable that, for lightly loaded compressor rows, the main benefit of endwall contouring would be similar to that for turbine rows, which is to reduce the cross-flow and the SKE.

\subsubsection{Experimental and Computational Investigations}

As mentioned previously, investigations of the effect of non-axisymmetric endwall contouring have mainly been performed on turbine blade rows and studies on compressor blade rows are limited.

Harvey (2008) performed experimental and computational studies on the application of non-axisymmetric endwall contouring on a linear compressor cascade at both design and offdesign values of incidences. The experimental study showed that the application of a contoured endwall resulted in a slightly improved exit flow field caused by a modest reduction in the local losses and in the flow underturning in the secondary flows region. CFD studies were performed and compared to the experimental data. It was shown that, at design 
incidence, the CFD results provided very good agreement with the experimental measurements and a reasonable match with the off-design measurements. Following his studies, Harvey (2008) concluded that the profiled endwall design used was not optimized as only modest benefit were observed, but he identified a flow mechanism that could mitigate the hub corner stall and justify the application of contouring. The flow mechanism consists of increasing the overturning of the secondary flows, which results in a stronger endwall crosspassage flow and in an enhanced migration of the secondary flows onto the suction surface of the blade in the spanwise direction. This flow mechanism was shown as a potential mean of controlling and mitigating the corner stall and was investigated by Harvery and Offord (2008).

Harvey and Offord (2008) performed a numerical investigation of the effect of nonaxisymmetric endwall contouring on a six-stage high-pressure compressor. The profiled endwall was applied on the hub of each of the rows, except the first and last rotor rows. In this study, the contouring was used as a means of corner stall suppression. It was found that the contouring successfully resulted in a suppression of the hub corner stall in each stator rows with significant reduction in the exit whirl angles and in the loss coefficients for the majority of the stator rows. In their study, only two different designs of profiled endwall were used and they concluded that the optimization of each row individually could provide additional benefit.

Reising and Schiffer (2009a) presented a numerical study of the potential aerodynamic benefit of the application of non-axisymmetric endwall contouring in axial transonic compressors. The initial study was performed on a transonic compressor stator blade with endwall profiling on the hub only. The application of endwall contouring resulted in a stronger cross-passage flow (i.e. higher secondary kinetic energy) and in a change in static pressure distribution on the suction surface of the blade due to the migration of the secondary flows. The contouring also improved the uniformity of the outlet flow angle. The results of their initial study showed significant improvement in performance at the design point with an increase in efficiency of $1.8 \%$ due to the suppression of the hub corner stall. However, Reising and Schiffer found an increased region of reverse flow at the shroud, which led them to perform an additional study which included the optimization of the shroud endwall along 
with the already optimized hub endwall. The results from their second study showed an additional $0.03 \%$ increase in efficiency at design incidence, but the real benefit was observed over the entire off-design range of values of incidence where a significant reduction in flow separation at the tip endwall was observed. The result of having both endwalls contoured showed an increase of $0.67 \%$ in efficiency at the off-design condition used for the casing endwall optimization.

Lu et al. (2009) conducted a numerical study on the effects of endwall contouring on an axial flow compressor stage and their conclusions are similar to those from the studies presented previously. The endwall profile studied is a combination of axisymmetric contouring in the axial direction and non-axisymmetric contouring in the pitchwise direction applied on both endwalls. The results showed that the contouring modified the cross-passage pressure gradient in such way that the outlet flow deviations were reduced and the hub corner stall significantly mitigated.

Lepot et al. (2011) performed a numerical investigation of the effect of blade sweep and non-axisymmetric hub profiling on a highly-loaded low-pressure compressor blade (diffusion factor $D=0.46$ ). The simulations were performed on a 1.5 stage compressor, which included inlet guide vanes, rotor blades and stators, but the optimization was only performed for the rotor blades. From their optimization work, two configurations showed benefits: a backward swept blade and a forward swept blade, both with non-axisymmetric endwall contouring. The backward swept blade improved the efficiency at the design point by unloading the midspan section of the blade, but resulted in the corner stall developing earlier. The design of the endwall profile was then optimized to enhance the cross-flow in order to mitigate the corner stall and improved the stability. The forward swept configuration was shown to relieve the hub loading and improved operability and the optimized endwall profile showed some benefit in reducing the shock losses. Although both configurations showed potential benefit, the former was selected to be experimentally investigated further. 


\subsubsection{Conclusions}

From the studies discussed in the latter part of this chapter, it can be concluded that many efforts have been put on the three-dimensional modification of the static pressure field near the endwall as a means of secondary flow control and secondary losses reduction in order to improve the component efficiency. Numerical and experimental investigations have been performed on compressor cascades with endwall contouring at both design and offdesign values of incidence and the results showed potential benefits resulting from the contouring. However, these studies were done on fairly highly-loaded compressor cascades with diffusion factors between 0.35 and 0.50 , in a region where the total losses only increased gradually with diffusion factor, as it was shown on Figure 2.3. The experimental research presented in the subsequent chapters adds to the previous studies by exploring the effect of non-axisymmetric endwall contouring on a more highly-loaded compressor cascade with a diffusion factor of 0.52 . The investigations performed at both design and off-design values of incidence and the oil surface flow visualisations on both the blade and the endwall are expected to provide a better understanding of the flow physics with the application of endwall contouring as a means of secondary flow control. 


\section{Chapter 3}

\section{Experimental Methods and Data Reduction Procedures}

\subsection{Introduction}

This chapter focus on the experimental methods and data reduction procedures that were used in the present experimental investigation conducted using an open-circuit wind tunnel and a modular test section in the Low-Speed Turbomachinery Laboratory (LSTL) at Carleton University. Recently, the facility has been used for investigations of compressor secondary flows by Ellison (2005) and for investigations of turbine secondary flows by Benner (2003), Zoric (2006), Knezevici (2011) and MacIsaac et al. (2010). Thus, the operating procedures and experimental repeatability are well established.

The chapter starts with a description of the wind tunnel, the test section and the research cascades. Next, the experimental instrumentation and the probe calibration apparatus are discussed, followed by a description of the pressure probes used and their uncertainties. Finally, the cascade testing procedures are summarized.

\subsection{Low-Speed Wind Tunnel}

An open-circuit wind tunnel, shown on Figure 3.1, was used for the experimental investigations conducted by the author. It was originally commissioned by Rodger (1992). 
The wind tunnel is used as an air supply for the test section. The wind tunnel consists of a centrifugal fan, a honeycomb flow straightener, a wide angle diffuser, a settling chamber and a contraction.

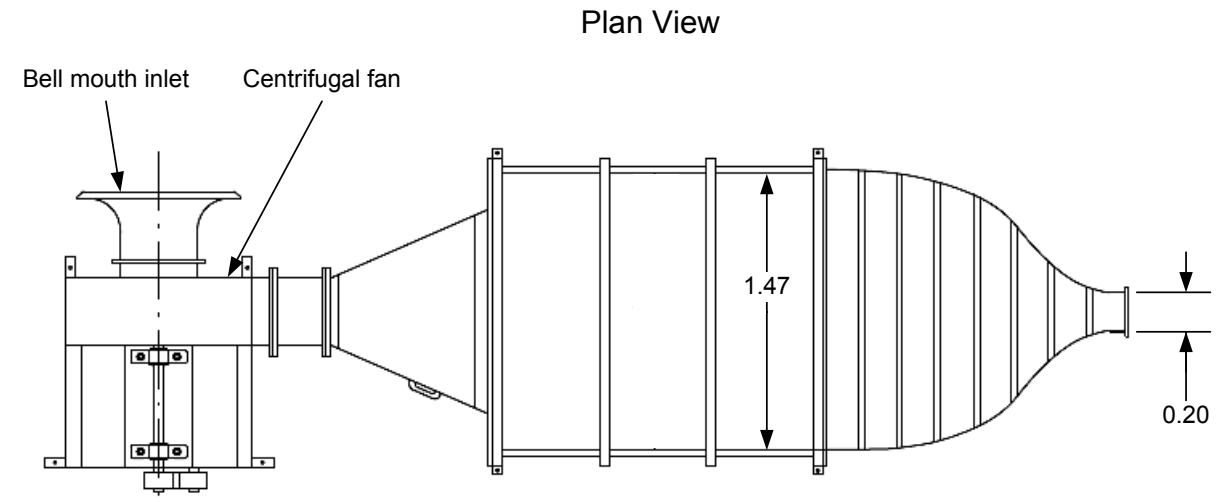

Elevation View

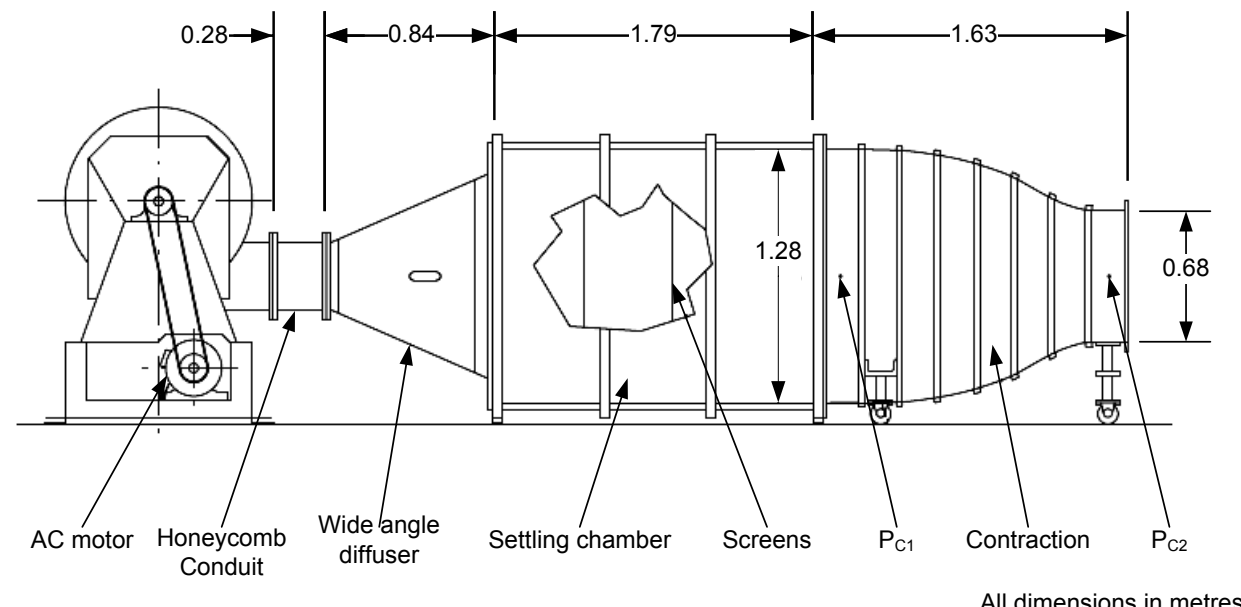

Figure 3.1: Wind-tunnel schematic (adapted from Knezevici (2011) and Benner (2003))

Air is drawn into the wind tunnel via a bellmouth inlet by the centrifugal fan (Daltec Industries, model number IE17P). The fan is driven by a $40 \mathrm{HP}$ AC motor controlled by a Mitsubishi variable frequency drive.

The flow leaving the centrifugal fan is highly non-uniform. Therefore, it passes through a honeycomb conduit to remove the swirl present and then into a wide angle diffuser to reduce its velocity before entering the settling chamber. The diffuser has a divergence angle of $19^{\circ}$ and contains five evenly spaced screens used to prevent boundary layer separation and to improve the uniformity of the flow. The settling chamber contains four 
screens used to further improve the uniformity of the flow and to reduce the large scale turbulence. The flow leaving the settling chamber is nearly stagnant and is accelerated through a square to rectangular contraction (with an area ratio of 14:1) into the test section (Knezevici, 2011; Rodger, 1992).

The wind tunnel operating point is set using the contraction pressures $P_{C 1}$ and $P_{C 2}$, measured from static taps located upstream and downstream of the contraction, respectively. The pressure difference $P_{C 1}-P_{C 2}$ is used to normalize non-dimensional coefficients and the pressure $P_{C 2}$ is used as the reference pressure for the transducers. The contraction pressure difference is sampled at the same frequency and for the same amount of time as the pressure probes. The wind tunnel operating point will be further discussed in Section 3.9.1.

\subsection{Test Section}

The present experiment was completed using the variable incidence linear cascade test section shown on Figure 3.2. The test section was designed and commissioned by Goobie (1989) and is connected to the wind tunnel described in Section 3.2.

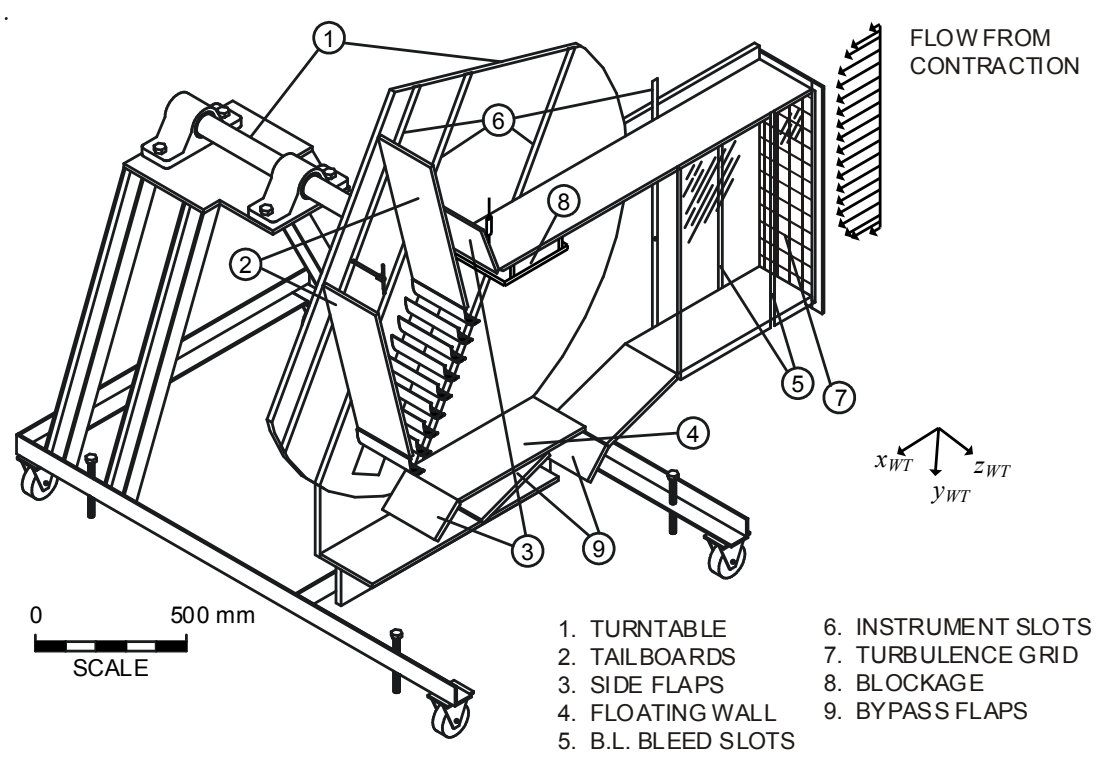

Figure 3.2: Linear cascade test section (adapted from Benner, 2003) 
The flow from the contraction entering the test section goes through a turbulence generating grid (item 7) which intent is to approximate engine representative levels of freestream turbulence intensity (FSTI) and length scale (Knezevici, 2011). The grid produces a FSTI of $4 \%$ and integral length scale of $0.4 \mathrm{~B}_{\mathrm{x}}$ (Mahallati, 2003). The $4 \%$ turbulence intensity is lower than engine levels, but is high enough that there should be representative turbulence effects. The inlet of the test section is rectangular and measures $200 \mathrm{~mm}$ by 673 $\mathrm{mm}$. Downstream of the grid are two boundary layer bleed slots (item 5) used to thin the endwall boundary layers entering the cascade.

The linear cascade is mounted on a turntable (item 1) which acts as the back endwall of the test section. The front endwall (not shown) is made of Plexiglas and is mounted on wheels to allow visual and physical access of the test section. Seven control surfaces and one blockage mechanism are used to set the inlet flow uniformity and outlet flow periodicity. The side flaps (item 3) and floating wall (item 4) are used to ensure that the inlet flow is uniform. The blockage (item 8) was designed by Mahallati (2003) to improve the uniformity of the flow at the top of the cascade. Two tailboards (item 2) located downstream of the cascade are used to set a periodic outlet flow. Bypass flaps (item 9) are mainly used to control the mass flow rate going through the linear cascade. The current test section was modified from previous experiments to accommodate compressor cascade testing. In addition to the existing control surfaces, two small tailboards (not shown) were added at the top of the existing tailboards (item 2) to act as a throttle. The throttling was needed to maintain the pressure upstream of the cascade above the ambient pressure. This is necessary for the functioning of the control surfaces located upstream (items 3,4 and 8), as well as the bleed slots (item 5). The throttle opening area was selected such that the pressure difference between the inlet of the cascade and the ambient air was similar to that present in the turbine cascade experiments. It was verified experimentally that the distance from the outlet of the cascade to the throttle was sufficient that the upstream effect of the throttle did not affect the flow in the cascade.

Flow measurements in the test section were performed using pitot and multi-hole pressure probes. The probes were attached to a stem installed either upstream or downstream of the cascade. The stem passes through the instrument slots (item 6) on the turntable where 
it is connected to the traverse mechanism. Details about this mechanism are further addressed in Section 3.6.5.

\subsection{Research Cascades}

The present research was completed using a compressor cascade designed by Pratt \& Whitney Aircraft (PWA). The cascade, designated CEGV-1, is designed to represent a compressor exit guide vane of an aircraft engine. The cascade midspan geometry and cascade nomenclature can be seen on Figure 3.3 and Table 3.1.

The cascade Cartesian coordinate system used for this research is defined such that the $\mathrm{x}$-axis, $\mathrm{y}$-axis and $\mathrm{z}$-axis are aligned with the axial, pitchwise and spanwise directions, respectively. The origin of the coordinate system is located at the blade leading edge. For axial plane measurement, the origin of the z-axis is located on the back endwall of the test section.

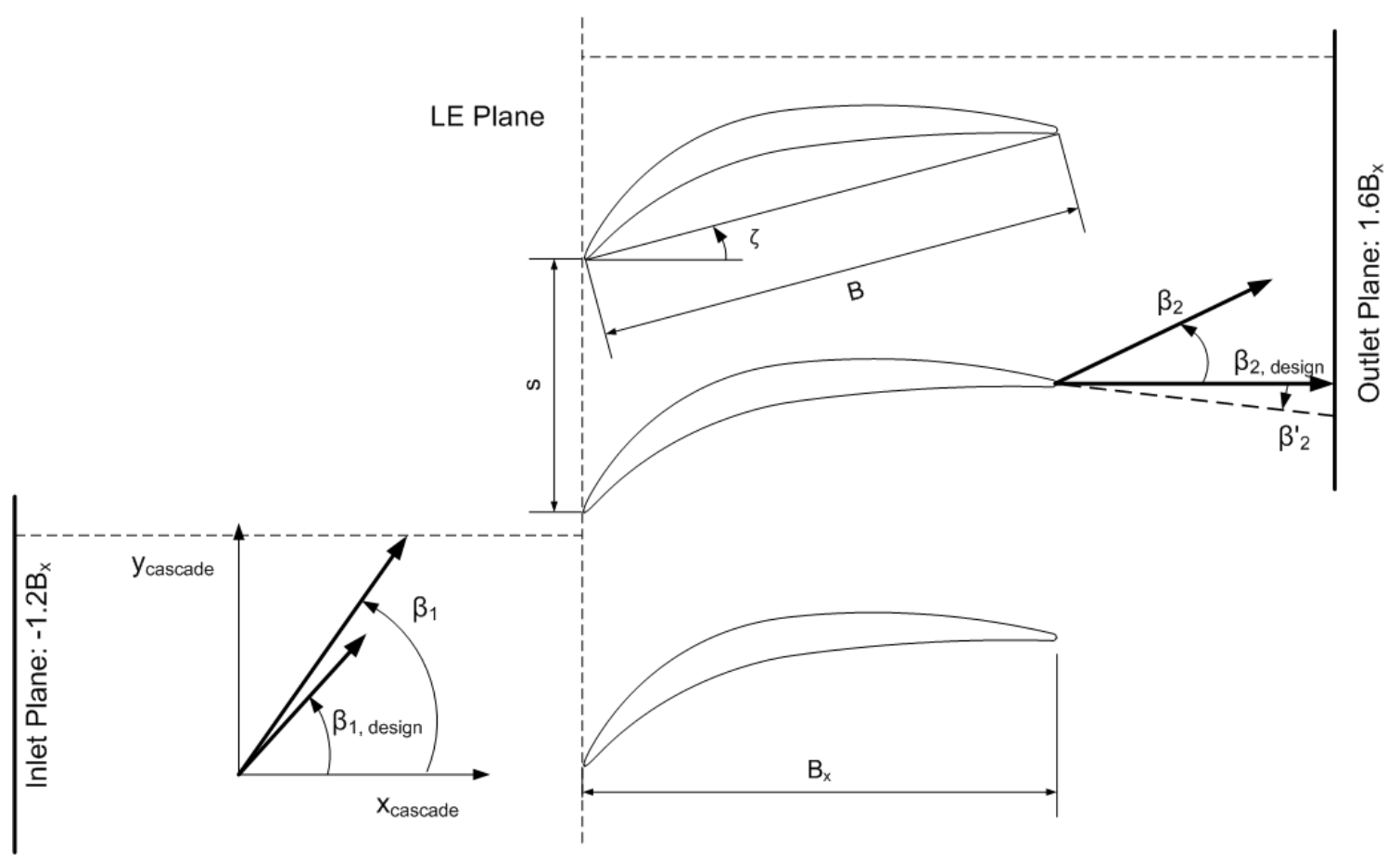

Figure 3.3 Cascade nomenclature and midspan geometry 
Table 3.1: Cascade nomenclature

\begin{tabular}{llll}
\hline \hline$\beta_{1, \text { design }}$ & design inlet flow angle & $\beta_{1}$ & actual inlet flow angle \\
$\beta_{2, \text { design }}$ & design outlet flow angle & $\beta_{2}$ & actual outlet flow angle \\
$\mathrm{B}$ & true chord & $\zeta$ & stagger angle \\
$\mathrm{s}$ & pitch spacing & $\mathrm{B}_{\mathrm{x}}$ & axial chord \\
$\beta^{\prime}{ }_{2}$ & blade outlet metal angle & & \\
\hline \hline
\end{tabular}

The location of the upstream and downstream flow field measurements can also be observed on Figure 3.3. All upstream measurements were conducted at 1.2 axial chords ahead of the cascade leading edge and the downstream measurements were made at 1.6 axial chords downstream of the leading edge. The geometric information and design parameters of the compressor cascade are given in Table 3.2.

Table 3.2: Cascade geometry

\begin{tabular}{lc} 
& CEGV-1 \\
\hline \hline Design inlet flow angle $\left(\beta_{1, \mathrm{des}}\right)\left[^{\circ}\right]$ & 47.8 \\
Design outlet flow angle $\left(\beta_{2, \mathrm{des}}\right)\left[^{\circ}\right]$ & 0 \\
True chord $(\mathrm{B})[\mathrm{mm}]$ & See Fig. 3.4 \\
Blade span $(\mathrm{h})[\mathrm{mm}]$ & 203.2 \\
Axial chord $\left(\mathrm{B}_{\mathrm{x}}\right)[\mathrm{mm}]$ & 94.0 \\
Pitch spacing $(\mathrm{s})[\mathrm{mm}]$ & 50.4 \\
Solidity $(\sigma=\mathrm{B} / \mathrm{s})$ & 1.93 \\
Stagger angle at midspan $(\gamma)\left[^{\circ}\right]$ & 14.9 \\
Trailing edge thickness $[\mathrm{mm}]$ & 1.27 \\
Number of airfoils in cascade & 10 \\
Diffusion factor & 0.52 \\
\hline \hline
\end{tabular}

The compressor cascade consists of ten blades and nine passages. At design incidence, the inlet and outlet flow angle are $47.8^{\circ}$ and $0^{\circ}$, respectively. Measurements are taken from the seventh passage starting from the top of the cascade. This passage was selected because the inlet flow at midspan was at the design incidence and it showed good inlet uniformity 
with its two adjacent passages. The middle passage could not be used because of the reduced quality of the flow at the top of the passage, as it will be discussed in Section 3.9.2.

The blades and the contoured endwall manufacturing process was stereo lithography (i.e. referred to as SLA). The blades and endwall were built using a standard photopolymer resin. The cascade blades are bowed near the two endwalls, such that the angle between the endwall and the suction surface is obtuse. This type of bow is known as positive bow. This feature was incorporated by PWA to contribute, along with the endwall contouring, to the reduction of secondary losses. Lewis and Hill (1971) provide details on the fundamental principles behind the application of bow (also called dihedral) as a mean of improving compressor aerodynamic performance. Positive bow on the blade is usually beneficial near the endwall as it imposes body forces on the fluid and increases the spanwise pressure gradient acting towards midspan, which will reduce any tendency for the secondary flows to cause separation in the corner region (Harvey, 2008). The endwall fluid in the blade and endwall corner will tend to migrate more strongly towards midspan due to this pressure gradient.

The blades have a constant axial chord length of $94.0 \mathrm{~mm}$ but as a result of the bowing the true chord length increases as the endwalls are approached. The true chord at midspan is $97.4 \mathrm{~mm}$ and the spanwise variation is shown in Figure 3.4. As seen, the true chord increases by about $2.2 \%$ from midspan to the endwall.

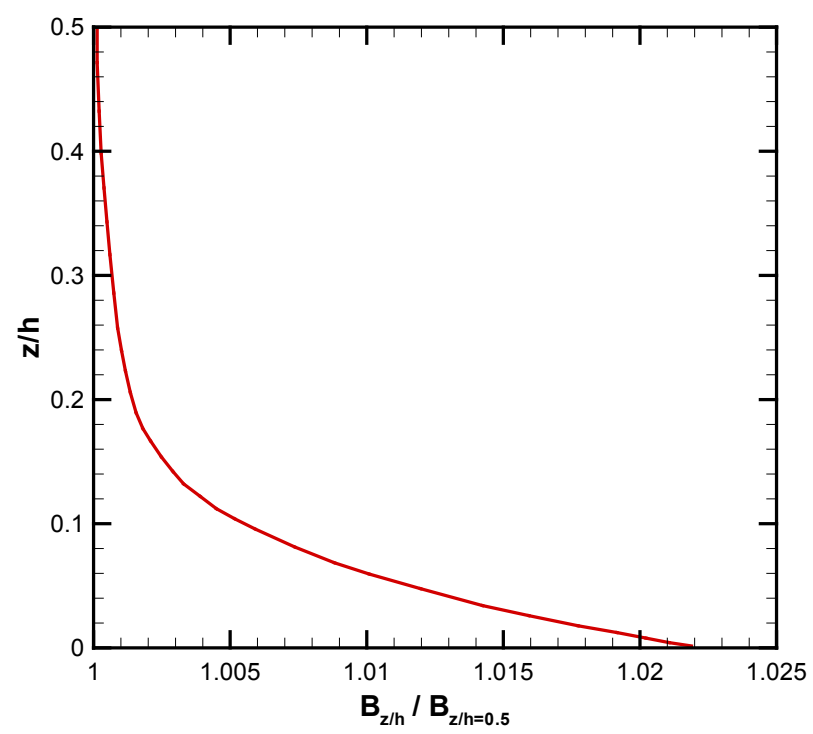

Figure 3.4: Variation of blade true chord along the span (Private Communication, PWA) 
The incidence of the cascade and the flow deviation angle at the inlet and exit of the cascade are defined as:

$$
\begin{array}{ll}
\text { Incidence } & i=\beta_{1}-\beta_{1, \text { des }} \\
\text { Deviation angle } & \delta=\beta_{2}-\beta_{2, \text { des }}^{\prime}
\end{array}
$$

Positive incidence results in more lift being generated and positive deviation results in underturning of the flow relative to the metal angle. All data presented in Chapter 4 are taken at zero incidence (i.e. $\beta_{1}=\beta_{1, \text { des }}=47.8^{\circ}$ ) and all data presented in Chapter 5 are taken at 7.0 degrees of incidence (i.e. $\beta_{1}=\beta_{1, \text { des }}+i=54.8^{\circ}$ ).

Two types of endwall configurations were tested during the course of this research: a basic flat metal endwall and a contoured endwall. The latter was designed and provided by PWA. The objective of the profiled configuration is to reduce the secondary losses across the cascade, thus reducing the total losses. Chapter 4 investigates the effect of the endwall contouring on the secondary losses at design inlet flow angle and Chapter 5, at 7.0 degrees of incidence.

To measure the airfoil surface static pressures, two airfoils were instrumented with static taps and internal pressure channels: one on the pressure side and one on the suction side. The internal pressures channels were designed by the author and Gordon MacIsaac ( $\mathrm{PhD}$ candidate). Bulged tubing were inserted in the channels and connected to a scani-valve using flexible tubing. The scani-valve was then connected to a differential transducer, also using flexible tubing, in order to measure the static pressures on the blade surfaces. The vanes were instrumented with three chordwise rows of 15 static taps each, located at 5,20 and $50 \%$ span. The taps at 5 and $20 \%$ span were located at the same chordwise positions as their corresponding tap number at midspan. All three taps at the same chordwise position were connected to a single internal pressure channel and pressure transducer. In order to measure the static pressures at a single spanwise position, a fine line of packing tape was affixed on the two chordwise rows not measured to block the taps. This ensured that the pressure transducer would read a pressure coming from a single tap. The process was repeated for each different row of static taps. 


\subsection{Endwall Contouring}

Recent studies have been investigating the application of endwall contouring as a method of secondary losses reduction. The surface curvature created by the application of contouring locally affects the cross-passage pressure gradient, thus affecting the flow field in the endwall region. For a turbine cascade, the main objective of endwall contouring is to reduce the secondary losses. This can be done by reducing the strength of the cross-passage flow and retarding the development of the passage vortex (Taremi and Sjolander, 2011; Knezevici et al., 2010; Praisner et al., 2007). For a compressor cascade, in addition to reducing secondary losses, it is also desired to reduce or remove the corner stall, as this is a major source of loss. The mitigation of the corner stall is achieved by forcing the flow to migrate off the endwall onto the blade suction surface, thus reducing the accumulation of low momentum fluid in the suction corner (Harvey and Offord, 2008). In contrast to turbine cascades, it is desired to increase the strength of the cross-passage pressure gradient to control the corner stall and thereby, reduce the secondary losses.

Figure 3.5 shows the rendering of the CEGV-1 contoured endwall. The contoured endwall is comprised of four dominant geometric features. There are two convex and two concave curvatures. A convex curvature will locally accelerate the flow, thus reducing the local static pressure. A concave curvature will locally diffuse the flow, thus increasing the local static pressure.

The first convex curvature, denoted $\mathrm{H}_{1}$, extends throughout the passage parallel to the pressure surface of the blade. This convex surface is used to locally accelerate the new boundary layer across the blade passage from the pressure side to the suction side. The second convex curvature, denoted $\mathrm{H}_{2}$, occupies the majority of the passage spacing near the trailing edge axial plane.

The first concave curvature, $T_{1}$, spans from the leading edge plane to approximately $\mathrm{x} / \mathrm{B}_{\mathrm{x}}=0.6$ and from $\mathrm{H}_{1}$ to the endwall/suction surface junction of the adjacent blade. This concave curvature is the main geometric feature in this contoured endwall. The last concave curvature, $\mathrm{T}_{2}$, is located on the endwall along the pressure surface of the blade. 


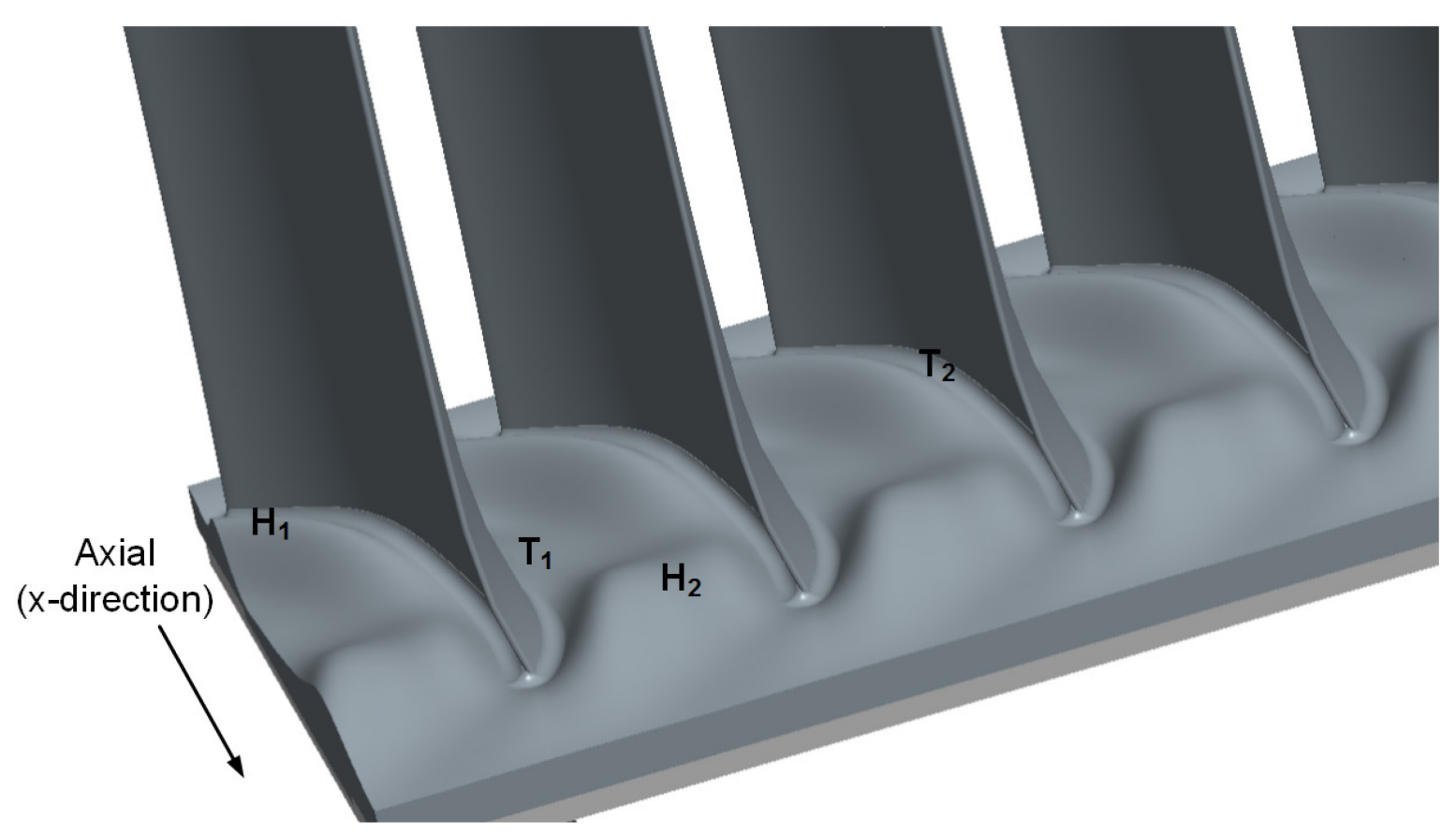

Figure 3.5: Rendering of CEGV-1 contoured endwall with major geometric features identified

The maximum positive and negative spanwise deflections of the endwall with respect to the nominal position are equivalent to $\mathrm{z} / \mathrm{h}=0.042$ and $\mathrm{z} / \mathrm{h}=-0.030$, respectively.

The effects of these different geometric features will be discussed in more details in Chapters 4 and 5.

\subsection{Experimental Instrumentation}

Prior to the beginning of this research, the data acquisition system was modified by Gordon MacIsaac (PhD candidate) from the existing system developed by Knezevici (2011) and Ellison (2005). A new data acquisition card was bought and new data acquisition software and a graphical user interface (GUI) were developed using the MATLAB programming language.

\subsubsection{Data Acquisition System}

The instrument voltage were recorded using a National Instruments data acquisition card (DAQ), model number NI-PCI-6229, connected to the PCI slot of the computer. The 
card has sixteen analog input channels with non-simultaneous differential sampling and with an analog-to-digital (ADC) resolution of 16 bits, a maximum sampling rate of 250,000 samples per second and a voltage range of $\pm 10 \mathrm{~V}$. For the present research, a maximum of eight differential channels were used on the card.

The measured voltages are transmitted to the DAQ computer via BNC cables connected to two BNC Terminal Blocks and are processed using data acquisition software developed in-house. The software was developed in MATLAB by MacIsaac and tested by the present author. It has the capability to record the instrument voltage outputs from the pressure transducers (Section 3.6.2), the temperature monitor (Section 3.6.3) and the digital barometer (Section 3.6.4). The software has a built-in function to calculate the Reynolds number, which is used to set the operating point of the wind tunnel and another built-in function to control the traverse mechanism (Section 3.6.5) used to move the probe when performing a test.

\subsubsection{Pressure Transducers}

Pressure measurements were made using Data Instruments differential pressure transducers (ASG DRAL501DN) having a full-scale operating range of $\pm 1250 \mathrm{~Pa}$. Knezevici (2011) quotes an estimated uncertainty of $\pm 2 \mathrm{~Pa}$ for the pressure recorded due to calibration, nonlinearity and hysteresis. For the present experiment, seven transducers were used for the probes pressure ports and one transducer was used for the wind tunnel contraction pressure difference $\left(\mathrm{P}_{\mathrm{C} 1}-\mathrm{P}_{\mathrm{C} 2}\right)$. Each of the pressure channels was sampled at a frequency of $1 \mathrm{kHz}$ for ten seconds, resulting in 10,000 samples per channel for each data point recorded.

The calibration of the transducers is done in-house using the technique described in Benner (2003). It consists on applying pressure to the differential pressure transducers using a water micromanometer. Pressures were applied to cover the operating range of the transducers. For each data point, the height difference between the two water columns $(\Delta \mathrm{h})$ and the voltages read by each transducer were recorded and linear relationships were derived from the results. The relationship between the pressure recorded and the voltage output $(\mathrm{E})$ is assumed to be linear with the form

$$
\Delta P=C_{1} E+C_{2}
$$


where $C_{1}$ is the calibrated slope of the response and $C_{2}$ is the zero pressure offset voltage. $C_{1}$ and $\mathrm{C}_{2}$ are obtained from a least-squares fit to the calibration data. Calibration data for the pressure transducers are given in Appendix A.

\subsubsection{Temperature Monitor}

An Omega i-series temperature meter, model DPi8-C24 with a type-T (CopperConstantan) thermocouple are used to measure the static temperature at the inlet of the cascade. The sampling frequency of the temperature measurement is $3 \mathrm{~Hz}$ with an estimated accuracy of $\pm 0.4^{\circ}$ as quoted by the manufacturer and reported by Mahallati (2003) and Knezevici (2011). The measured temperature is used to calculate the density and the dynamic viscosity (found using Sutherland's rule) inside the test section, which is used to set the wind tunnel operating point, as discussed in Section 3.9.1.

\subsubsection{Digital Barometer}

An Omega digital barometer, model DPI740, is used to measure the atmospheric pressure at a sampling frequency of $2 \mathrm{~Hz}$. The measurement uncertainty is quoted by the manufacturer as $\pm 0.2 \%$ of full scale range (Knezevici, 2011). The barometer has a precalibrated absolute pressure transducer with a range from 77 to $115 \mathrm{kPa}$. The ambient pressure is used to calculate the air density from the ideal gas law which is used to set the wind tunnel operating point.

\subsubsection{Stepper Motor Controller}

During a test, the spanwise and pitchwise positions of the probe are controlled by a two-axis linear traverse mechanism. The traverse mechanism is composed of traverse gears, two stepper motors and a Velmex VXM stepping motor controller. The controller is able to move one motor at a time. One revolution of the stepper motor consists in 400 steps and each step moves the probe by $0.003175 \mathrm{~mm}$. ASCII commands are defined in the data acquisition software and sent to the controller via one of the RS-232 serial ports of the DAQ computer. 


\subsection{Probe Calibration Apparatus}

The probes are calibrated in-house in the calibration apparatus shown on Figure 3.6. The apparatus consists of a motorized turntable mechanism designed by Yaras (1987) inside an open-circuit, suction-type calibration box designed by Benner (2003). Filtered ambient air is drawn through a bellmouth and accelerated in a convergent nozzle $(\mathrm{d}=55 \mathrm{~mm})$ by a suction-type radial blower. The blower is equipped with a throttle valve that controls the jet velocity to a maximum of approximately $45 \mathrm{~m} / \mathrm{s}$. The jet passes through the axes of rotation of the two turntables and leaves through a second bellmouth. The probe holder is designed such that the tip of the probe remains at the center of rotation of both axes as the probe rotates in yaw $(\alpha)$ and pitch $(\beta)$. The motorized turntables can rotate the probe through approximately $\pm 50^{\circ}$. Reference total $\left(\mathrm{P}_{\mathrm{C} 1}\right)$ and static $\left(\mathrm{P}_{\mathrm{C} 2}\right)$ pressures are measured upstream at the inlet bellmouth and at the nozzle exit, respectively. More information about the calibration procedures and data reduction can be found in Benner (2003) and Mahallati (2003).

The calibration apparatus was used to calibrate the three-hole and seven-hole probes used in the present research. The probes will be further described in Sections 3.8.2 and 3.8.3. 


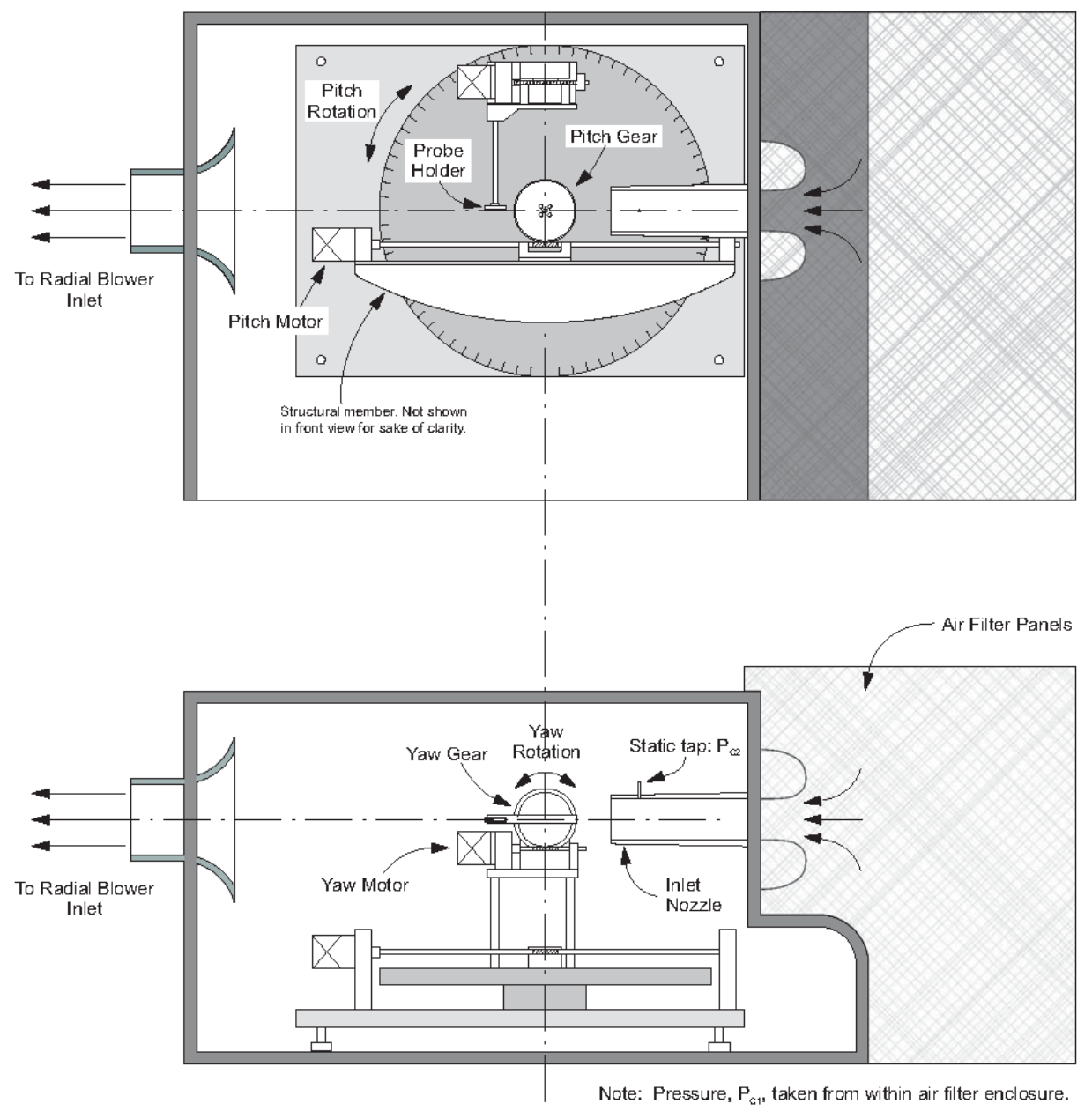

Figure 3.6 Schematic of probe calibration apparatus (from Benner, 2003)

\subsection{Pressure Probes}

\subsubsection{Pitot Tube}

An L-shape stainless steel Pitot tube was traversed $1.2 \mathrm{~B}_{\mathrm{x}}$ upstream of the leading edge of the cascade to measure the inlet endwall boundary layer. The Pitot tube has inner and outer diameters of 0.33 and $0.64 \mathrm{~mm}$, respectively. The diameter of the probe is approximately $2.7 \%$ of the boundary layer thickness, similar to numbers quoted by Benner (2003); therefore, it is assumed that good resolution of the boundary layer was achieved. The Pitot tube is 
installed on the traverse mechanism and is bent such that the tip touches the wall of test section first. Knezevici et al. (2010) quotes an uncertainty of $\pm 0.3 \%$ of the inlet midspan dynamic pressure for the total pressure readings.

\subsubsection{Three-Hole Probe}

A three-hole pressure probe, manufactured by Mahallati (2003), was used to measure the two-dimensional flow fields upstream of the cascade. The probe, as shown on Figure 3.7, is used to measure total and static pressures and flow angularity. The sign convention used for the present study defines a positive pitch angle when the flow is directed towards the probe hole 3 (refer to Figure 3.7). The probe is calibrated in the calibration apparatus described in Section 3.7. The three-hole probe was calibrated in $0.5^{\circ}$ increments from $-21^{\circ}$ to $+21^{\circ}$ at jet velocities of 15 and $25 \mathrm{~m} / \mathrm{s}$, corresponding to the cascade outlet and inlet nominal velocities, respectively. The calibration was done at two different jet velocities to detect the Reynolds number effect, which was found to be non-negligible at high values of pitch angle. Calibration and data reduction procedures are presented in Appendix B.

Mahallati (2003) quotes an estimated uncertainty of $\pm 0.5^{\circ}$ for the flow angle measured. The uncertainties quoted for the measured dynamic and total pressures are $\pm 0.5 \%$ and $\pm 0.25 \%$ of the inlet midspan dynamic pressure, respectively. The uncertainties are due to repeatability, transducer calibration, probe alignment and micro-fouling (Mahallati, 2003). Micro-fouling here corresponds to the accumulation of undesired substances on the tip of the probe (i.e. dirt), which affect the probe uncertainty. 

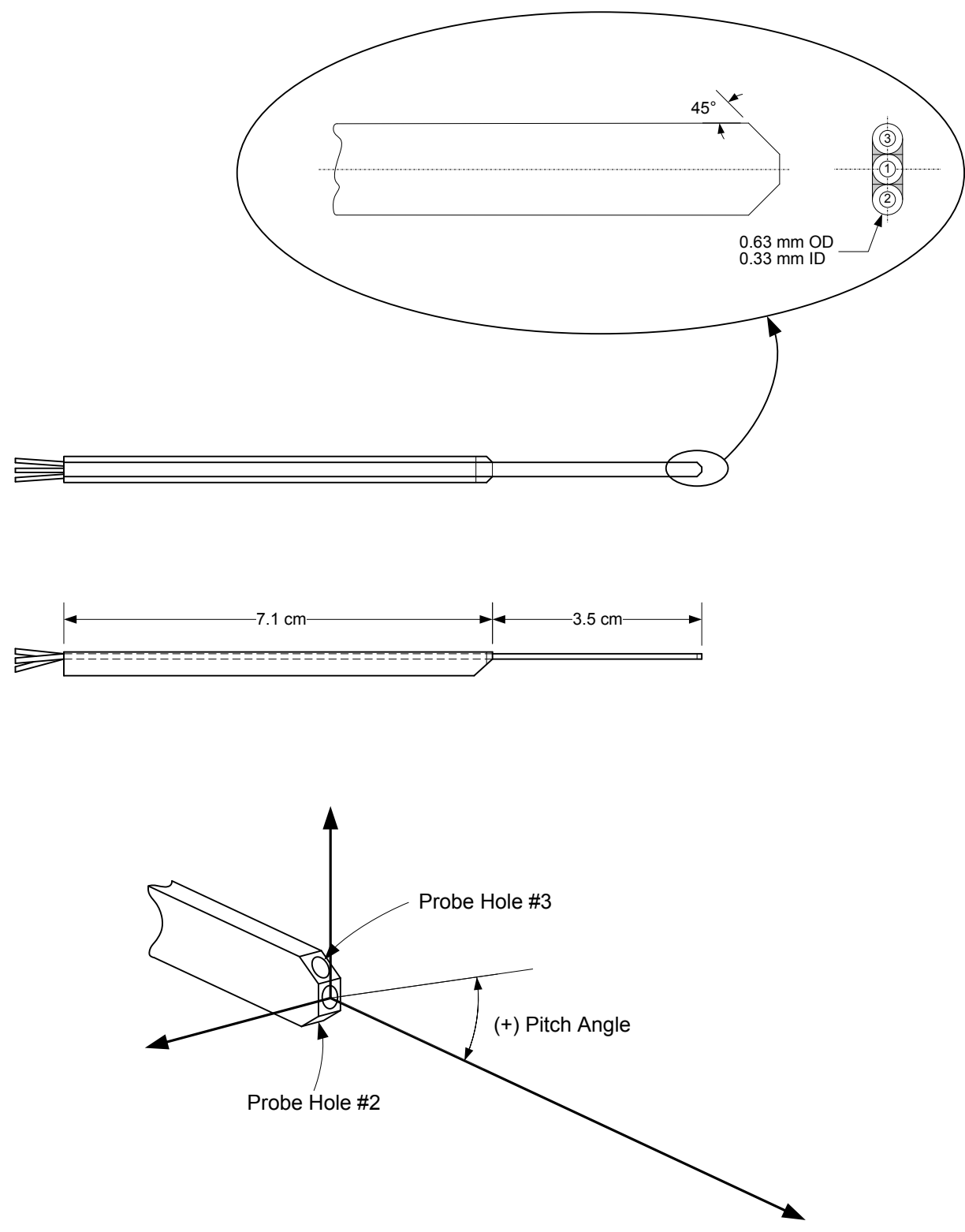

Figure 3.7: Three-hole probe (adapted from Mahallati, 2003)

\subsubsection{Seven-Hole Probe}

A seven-hole pressure probe, shown on Figure 3.8, was used to measure the threedimensional flow fields downstream of the compressor cascade. The probe was designed at 
Carleton University by Knezevici (2011). The probe was designed with a gooseneck tip and a mounting system to allow for intra-passage and near-wall measurements. Intra-passage measurements were not performed in the present study. The probe has a $60^{\circ}$ cone angle and a tip outer diameter (d) of $1.83 \mathrm{~mm}$, which corresponds to $\mathrm{d} / \mathrm{s}=0.036$ and $\mathrm{d} / \mathrm{h}=0.009$ relative to the present blade pitch (s) and span (h), respectively. The pressures measured at the seven ports of the probe are used to determine the steady total and static pressures, the flow yaw $(\alpha)$ and pitch $(\beta)$ angles, and the three components of velocity $(u, v, w)$ for each measured data point.

\section{TOP VIEW}

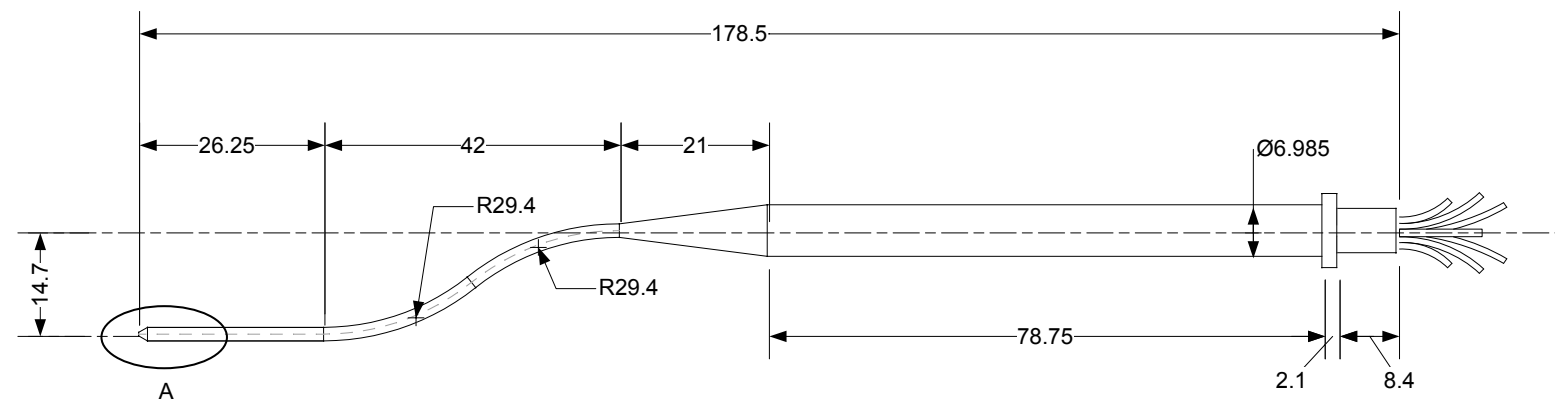

\section{A - ENLARGED}

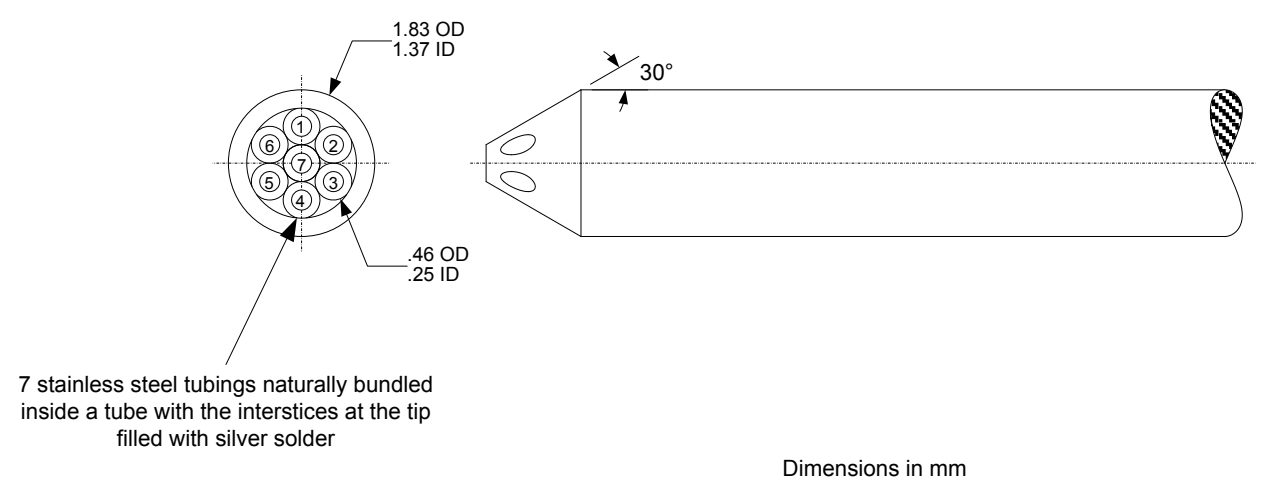

Figure 3.8: Seven-hole probe geometry (adapted from Knezevici, 2011)

The probe was calibrated over a pitch $(\beta)$ and yaw $(\alpha)$ angles range of $-50^{\circ}<\alpha, \beta<50^{\circ}$ in $2^{\circ}$ increments for nine different flow velocities $(5,10,15,20,25,30,35,40$ and $45 \mathrm{~m} / \mathrm{s})$. These jet flow velocities cover most velocity, and thus probe Reynolds number, ranges seen 
in the experiment. The seven-hole probe was again calibrated in the apparatus described in Section 3.7. The calibration and data reduction procedures used in this research were developed by MacIsaac et al. (2009) and by the present author, adapted from the methods of Benner (2003) and Gerner et al. (1984). The data reduction procedure uses an iterative process to interpolate between the data for the various calibration Reynolds numbers for the actual Reynolds numbers experienced by the probe. The resulting uncertainty in the local dynamic pressure is estimated to be $\pm 0.4 \%$ of the reference dynamic pressure (MacIsaac et al., 2010) rather than the $\pm 0.8 \%$ quoted by Benner (2003). A similar process was developed by the present author to allow extrapolation of the calibration data for local Reynolds numbers outside the calibrated range, as it occasionally occurred in the present measurements. Details about the calibration and data reduction procedures are given in Appendix C. The estimated uncertainty is $\pm 0.5^{\circ}$ for the measured flow angles and $\pm 0.4 \%$ of the inlet midspan dynamic pressure for the measured total pressures (Knezevici, 2011; MacIsaac et al., 2010).

Challenges in measuring total pressure in secondary flows using a seven-hole pressure probes have been discussed by MacIsaac et al. (2009). Some of the key factors affecting the pressure sensed by a probe and influencing the uncertainties are the Reynolds number, flow angularity, shear flow and turbulence levels (Chue, 1975). The probe used in this experiment is calibrated for Reynolds number and flow angularity but the two other factors are omitted. MacIsaac et al. (2009) investigated the effect of shear flow and turbulence levels on the probe response in different turbine cascades. Scribner (2011) developed a complex calibration procedure for the seven-hole probe in elevated turbulence fields. However, this procedure could not be applied here since it requires the turbulence to be measured in the flow field, which was not done in the present experiment. 


\subsection{Cascade Testing Procedures}

\subsubsection{Wind Tunnel Calibration and Operating Point}

The wind tunnel operating point of the present investigations was a constant Reynolds number of 150,000 based on the cascade inlet centerline velocity $\left(\mathrm{V}_{\mathrm{CL}}\right)$ and the blade axial chord $\left(B_{x}\right)$. Reynolds number is defined as:

$$
\operatorname{Re}=\frac{\rho V_{C L} B_{x}}{\mu}
$$

where $\rho$ is the air density and $\mu$ is the air dynamic viscosity calculated using the ambient air temperature and pressure (refer to Sections 3.6.3 and 3.6.4).

In order to calculate the centerline velocity and to set the wind tunnel operating point, the test section must be calibrated to relate its inlet conditions to the contraction pressure difference $P_{C 1}-P_{C 2}$ (Knezevici, 2011). The wind tunnel total and dynamic pressure coefficients are defined as:

$$
C_{P 0_{W T}}=\frac{P_{0}-P_{C 1}}{P_{C 1}-P_{C 2}}
$$

and

$$
C_{q_{W T}}=\frac{P_{0}-P}{P_{C 1}-P_{C 2}}
$$

where $P_{o}$ and $P$ are the total and static pressures on the inlet centerline of the cascade. These two coefficients are determined experimentally for each test case examined by traversing the three-hole pressure probe in the pitchwise direction at half-span, 1.2 axial chords upstream of the leading edge of the cascade. The measured total and dynamic pressures coefficients are pitchwise-averaged to single values to form the wind tunnel calibration coefficients for the test case.

The inlet centerline velocity, used to calculate the wind tunnel Reynolds number (equation (3.4)), is related to the dynamic pressure calibration coefficient as follows:

$$
V_{C L}=\sqrt{\frac{2 \cdot C_{q W T} \cdot\left(P_{C 1}-P_{C 2}\right)}{\rho}}
$$


The wind tunnel fan speed is adjusted to give the contraction pressure difference, $P_{C 1}-P_{C 2}$, that results in an inlet centerline velocity and Reynolds number matching the desired operating condition.

\subsubsection{Inlet and Outlet Flow Quality}

In a linear cascade, it is important to set the inlet uniformity and outlet periodicity of the flow prior to taking detailed measurements of the flow field. The purpose of having good uniformity and periodicity is to approximate effectively the infinite blade row that is present in an actual compressor.

The initial setup of the inlet control surfaces of the test section (side and bypass flaps, floating wall and blockage mechanism; refer to Section 3.3) were estimated positions to obtain the desired inlet angle. The tailboards were set slightly divergent from the design outlet flow angle to allow for the boundary layer growth on the tailboards. For each setup, the inlet centerline was traversed at $1.2 \mathrm{~B}_{\mathrm{x}}$ upstream of the leading edge of the cascade with the three-hole probe (refer to Section 3.8.2) to measure the resulting flow angularity and pressures. Once the inlet flow was aligned at the design inlet angle by adjusting the inlet control surfaces, the periodicity of the flow was examined at midspan $1.6 \mathrm{~B}_{\mathrm{x}}$ downstream of the leading edge using the seven-hole probe (refer to Section 3.8.3). If necessary, the outlet periodicity was adjusted using the tailboards. The process was repeated until both the inlet flow angularity and outlet flow periodicity were within the defined tolerances.

The inlet uniformity and outlet periodicity of the CEGV-1 compressor cascade on the flat endwall and at design incidence are shown on Figure 3.9, where $y / s=0$ is the top of the cascade. The flow angles and the dynamic, static and total pressure coefficients are presented for both inlet and outlet planes.

Challenges were encountered at setting the uniformity and periodicity at the top of the cascade. The compressor cascade studied was highly-loaded, which made it sensitive to small change in incidence. The blockage mechanism designed by Mahallati (2003) to improve the uniformity at the top of the cascade (see Section 3.3) showed limited improvement for the present investigation. However, the passage used for the detailed measurements (i.e. passage 
7 on Figure 3.9) and its adjacent passages (i.e. passages 6 and 8 on Figure 3.9) showed uniformity and periodicity within the desired tolerances.
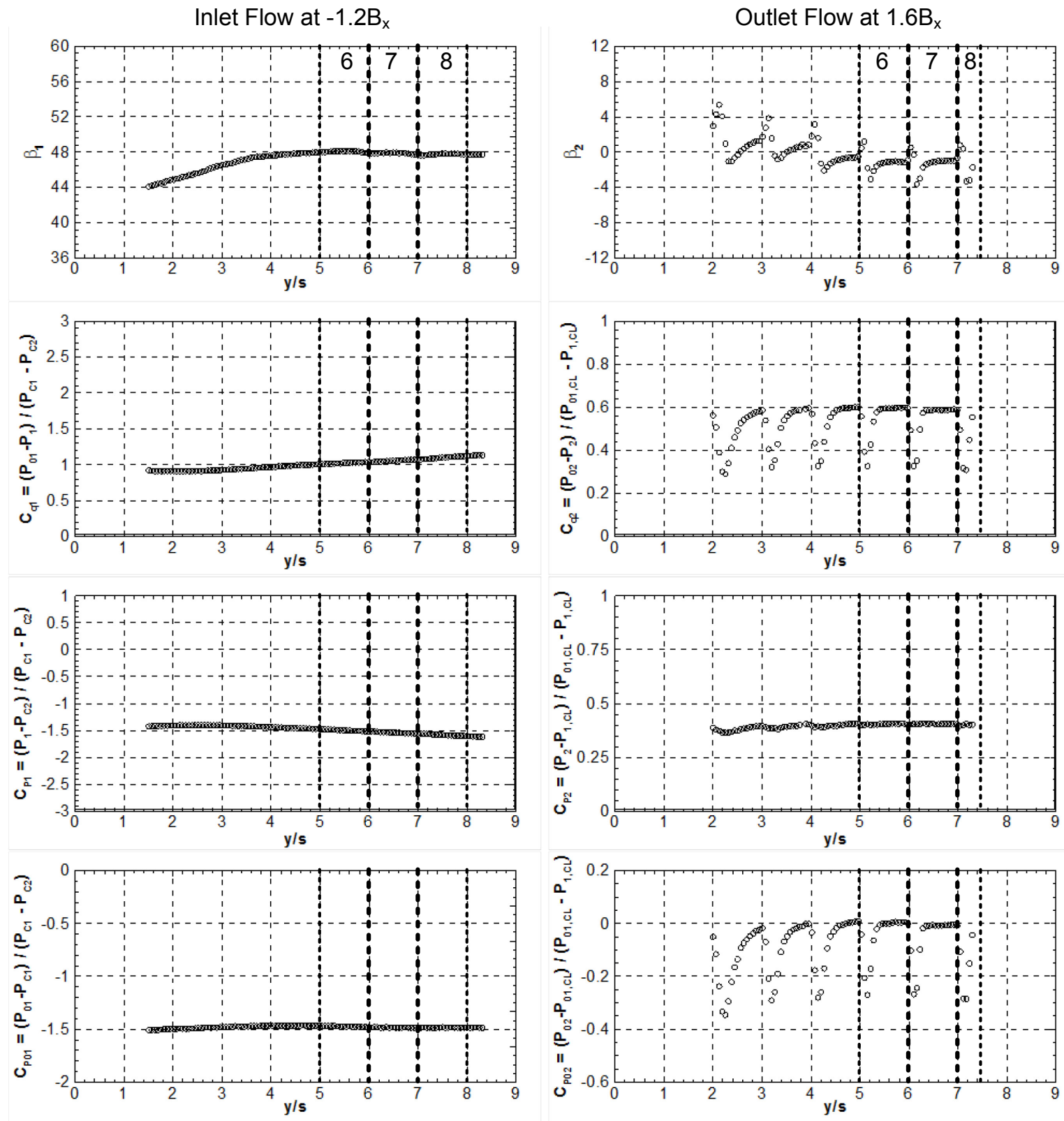

Figure 3.9: Inlet flow uniformity and outlet flow periodicity - flat endwall

A turbulence generating grid is located 18.4 axial chord lengths upstream of the centerline of the cascade. The documented freestream turbulence intensity and integral length scale are $4 \%$ and $0.4 \mathrm{~B}_{\mathrm{x}}$ for this grid (Mahallati, 2003). Different compressor cascades 
(Küsters et al., 1999; Köller et al., 2000; Schreiber et al., 2002) and turbine cascades (Zoric, 2006; Gregory-Smith and Cleak, 1992) investigators studied the effects of freestream turbulence intensity on the secondary flows showed that it had little influence in the range of the Reynolds numbers experienced in the present study. Based on these investigations, it is assumed that the freestream turbulence intensity has little effect on the secondary flows in the present study.

The inlet boundary layer was documented to determine its state and quantify the total pressure deficit. The inlet plane was traversed in the spanwise direction at nine pitchwise locations across two passages using the Pitot-tube described in Section 3.8.1. Figure 3.10 shows the results of the traverses, which were made at $0.25 \mathrm{~s}$ intervals, at 1.2 axial chord lengths upstream, from the leading edges of blades 5 to 7 .

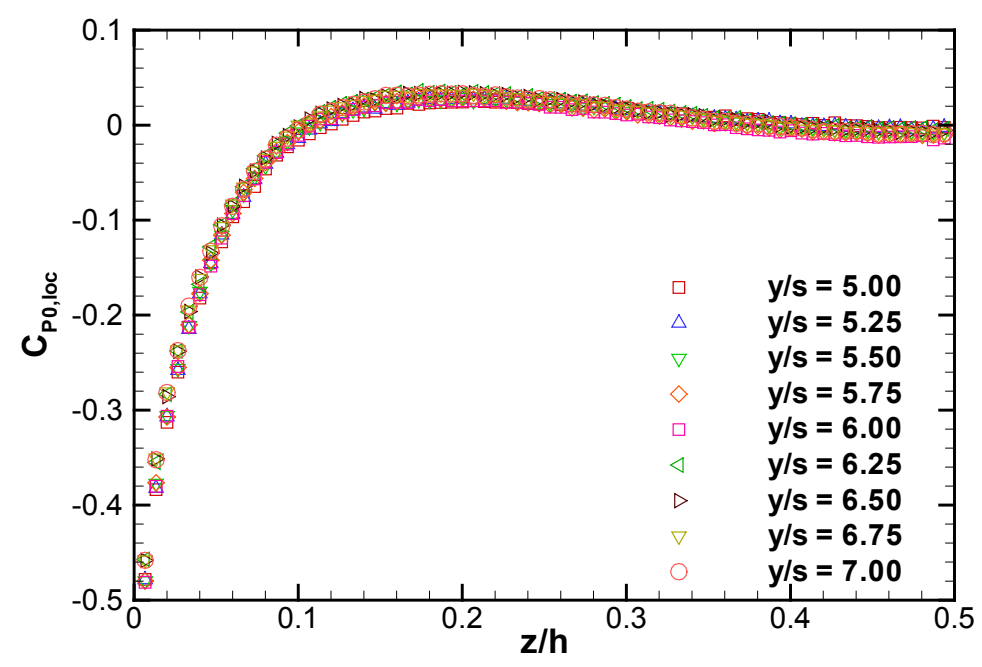

Figure 3.10: Inlet boundary layer profile measured at $-1.2 B_{x}$ and increments of $0.25 y / s$

Data on Figure 3.10 show minimal pitchwise variation of the profile. The inlet boundary layer profiles will be further discussed in Chapter 4 and Chapter 5. As noted by previous authors (Knezevici et al., 2008; MacIsaac et al., 2010), the turbulence generating grid used in the present experiment causes the inlet total pressure distribution to be nonuniform from the endwall to half-span. The inlet plane was also traversed using the threehole probe described in Section 3.8.2 to measure both the total pressure and flow angle distributions. The recorded total pressure was mass-averaged over one pitch and half span to 
provide the reference mass-averaged total pressure coefficient $\left(C_{P 0,1}^{\prime \prime}\right)$ used to calculate the losses through the compressor cascade. This topic will be further discussed in Section 3.9.4.1.

\subsubsection{Seven-Hole Probe Axial Traverses}

The downstream pressure measurements were performed using the seven-hole pressure probe described in Section 3.8.3 on an axial plane located 1.6 axial chords behind the leading edge of the cascade. The measurement matrix, shown in Figure 3.11, covers half the span and 2.2 blade pitches. Two passages (i.e. $y / s=5$ to 7 ) were initially traversed to document the outlet flow periodicity over the whole passage up to midspan. However, after the outlet flow periodicity was confirmed, only passage 7 (between blades 6 and 7) was traversed for the downstream flow field measurements presented in Chapter 4 and Chapter 5.

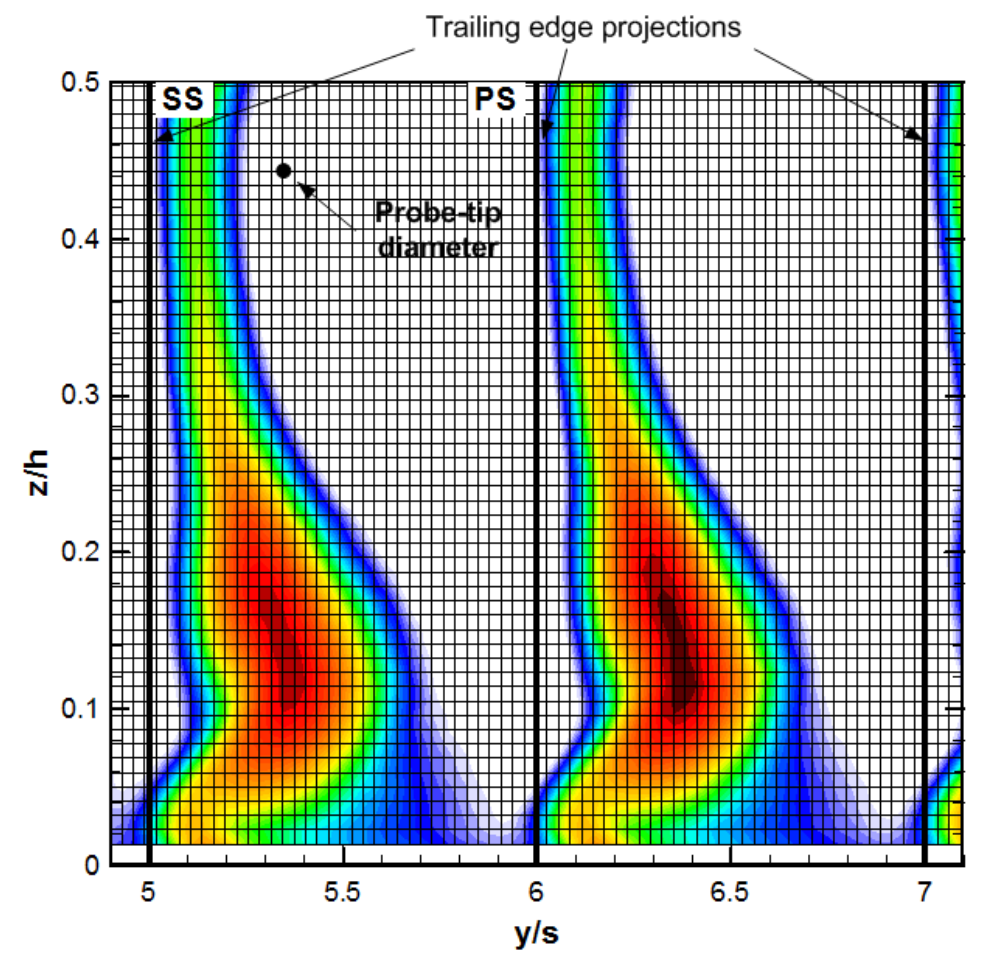

Figure 3.11: Measurement grid with trailing edge projection

The measurement matrix consists of 1904 grid points per pitch, for a total of 4201 . The pitchwise and spanwise intervals in the measurement grids were constant at approximately $2.9 \%$ and $0.9 \%$ of the blade spacing and blade span, respectively. The measurement grid density selected was similar to the ones used by previous investigators (MacIsaac et al., 
2009; Knezevici, 2011; Zoric, 2006; Benner, 2003). The probe-tip diameter is also shown to scale on Figure 3.11 to indicate low blockage represented by the probe.

The downstream measurements were taken at a sampling rate of $1 \mathrm{kHz}$ for ten seconds for a total of 10000 samples per measurement point. The average running time for a full downstream measurement grid was about 20 hours.

\subsubsection{Data Reduction}

Colour-flood contour plots, such as the one presented on Figure 3.11, provide a good qualitative impression of the downstream total pressure loss distributions. However, quantitative information about the losses can be gained from the overall area- and massaverages parameters. These integrated quantities are computed at cell centres of the grid shown on Figure 3.11 and have the following form (MacIsaac et al., 2010):

$$
\begin{array}{ll}
\text { Area-averaged: } & \bar{\psi}=\frac{1}{A} \int_{0}^{\frac{1}{2} h} \int_{0}^{s} \psi d y d z \\
\text { Mass-averaged: } & \psi^{\prime \prime}=\frac{\rho}{\dot{m}} \int_{0}^{\frac{1}{2} h} \int_{0}^{s} u \psi d y d z
\end{array}
$$

where $\psi$ is the parameter of interest. The values of $\psi$ corresponds to the arithmetic average of the corner values (i.e. measured data points). The integration limits are set from the wall to half span in the spanwise (i.e. z-component) direction and across one blade spacing in the pitchwise (i.e. $y$-component) direction. The use of these integrated parameters in the data reduction will be further explained in the following sections.

\subsubsection{Measured and Mixed-Out Losses}

The efficiency of a turbomachine is dependent on the entropy that it generates. The entropy is an extensive flow property and can not be directly measured in experiment. However, it can be related to the total pressure difference between two planes as shown in Section 3.5 of Knezevici (2011). This makes total pressure deficit a suitable parameter to quantify the losses across the cascade. 
The total pressure deficit coefficient $C_{P 0}$ represents the local total pressure loss relative to the upstream reference midspan value normalized on the upstream reference dynamic pressure at midspan. It is defined as:

$$
C_{P 0}=\frac{P_{0}-P_{0 C L, 1}}{q_{C L, 1}}
$$

As discussed in Section 2.2, for blade rows, losses are typically expressed in terms of an overall mass-averaged total pressure loss coefficient $\left(\Omega_{\text {Total }}\right)$, which is in turn decomposed into profile, secondary and tip leakage losses.

$$
\Omega_{\text {total }}=\Omega_{\text {profile }}+\Omega_{\text {secondary }}+\Omega_{\text {tip }}
$$

The present investigations were performed in absence of tip clearance, and thus $\Omega_{t i p}$ is zero. The measured mass-averaged total pressure loss coefficient is obtained from the following equation:

$$
\Omega_{\text {total }}=\left(C_{P 0,1}^{\prime \prime}-C_{P 0,2}^{\prime \prime}\right)\left(\frac{q_{C L, 1}}{q_{1}^{\prime \prime}}\right)
$$

where $C_{P 0,1}^{\prime \prime}$ and $C_{P 0,2}^{\prime \prime}$ are the mass-averaged total pressure deficit coefficients at the inlet and outlet planes, respectively. The difference in mass-averaged total pressure deficit coefficients is normalized by the mass-averaged dynamic pressure coefficient at the inlet plane $\left(C_{q, 1}^{\prime \prime}=q_{1}^{\prime \prime} / q_{C L, 1}\right)$. The mass averaged total pressure coefficients are defined as:

$$
C_{P 0}^{\prime \prime}=\frac{\int_{0}^{\frac{1}{2} h} \int_{0}^{s} \rho u(y, z) C_{P 0}(y, z) d y d z}{\int_{0}^{\frac{1}{2} h} \int_{0}^{s} \rho u(y, z) d y d z}
$$

The integration limits are from the endwall to half span over one blade pitch. The total pressure on the endwall is assumed to be equal to the static pressure at the nearest node to the wall and the velocity at the wall is set to zero.

The profile loss coefficient is obtained by integrating the total pressure deficit coefficient over one pitch at midspan (i.e. denoted as pitchwise mass-averaged):

$$
C_{P 0}^{\prime}=\frac{\int_{0}^{s} \rho u(y) C_{P 0}(y) d y}{\int_{0}^{s} \rho u(y) d y}
$$


The profile loss coefficient is then defined as:

$$
\Omega_{\text {profile }}=-C_{P 0,2}^{\prime}\left(\frac{q_{C L, 1}}{q_{1}^{\prime}}\right)
$$

where 2 represents the outlet measurement plane. It is assumed that the midspan total pressure deficit at the inlet plane is zero. The midspan flow is assumed to be uniform and unaffected by the spanwise non-uniformities discussed in Section 3.9.2.

One of the main objectives of the present experiment is to study the secondary losses in a compressor cascade. Thus, the secondary loss coefficient is of particular interest. It is obtained from equation (3.11), omitting the tip leakage losses:

$$
\Omega_{\text {secondary }}=\Omega_{\text {total }}-\Omega_{\text {profile }}
$$

It is important to note that the above equations define the losses generated up to the measurement plane. They are referred as measured losses. The measured losses are dependent of the axial location since losses continue to be generated downstream through mixing until the flow completely mixes out to uniform conditions (Knezevici, 2011). This is referred as the mixed-out condition. In order to be able to compare the results obtained from different research cascades using different traverse plane locations, mixed-out losses are used because they are independent of the location of the downstream measurement plane (Benner, 2003).

The mixing losses are found by applying the continuity and momentum equations to a control volume extending from the downstream measurement plane to the fully mixed-out plane over one blade pitch and from the endwall to half span (Knezevici, 2011; Zoric, 2006; Benner, 2003). It is assumed that the mixing occurs at constant area under incompressible conditions. It should be noted that the analysis neglects the endwall shear stresses acting on the control volume. Yaras and Sjolander (1989) investigated the influence of shear stresses on the mixed-out losses calculated from measurements made at different axial locations for the same cascade flow and concluded that the effects were small for measurements at locations larger than $1.40 \mathrm{~B}_{\mathrm{x}}$ (Benner, 2003). The present mixing calculations for the overall and profile losses use the procedure from MacIsaac et al. (2009), as adapted from Benner (2003). The full derivation can be found in Sections 3.9.1 of Benner (2003). The final forms for the total and profile mixed-out losses are: 


$$
\begin{aligned}
& \Omega_{\text {total,mixed }}=\left(C_{P 0,1}^{\prime \prime}-C_{P 0, \text { mixed }}^{\prime \prime}\right)\left(\frac{q_{C L, 1}}{q_{1}^{\prime \prime}}\right) \\
& \Omega_{\text {profile,mixed }}=-C_{P 0, \text { mixed }}^{\prime}\left(\frac{q_{C L, 1}}{q_{1}^{\prime}}\right)
\end{aligned}
$$

\subsubsection{Streamwise Vorticity and Secondary Kinetic Energy}

The streamwise vorticity, $\omega_{\mathrm{s}}$, and the secondary kinetic energy, SKE, are two useful additional parameters to examine when studying the three-dimensional flow structures downstream of a linear cascade.

The vorticity is defined as the curl of the velocity vector, which has the following form:

$$
\bar{\omega}=\nabla \times \bar{V}
$$

The streamwise vorticity is a projection of the total vorticity in the mean direction of the flow and is defined as:

$$
\omega_{s}=\omega_{x} \cos \overline{\bar{\beta}}_{2}+\omega_{y} \sin \overline{\bar{\beta}}_{2}
$$

where the axial $\left(\omega_{x}\right)$ and pitchwise $\left(\omega_{y}\right)$ components of vorticity are

$$
\begin{aligned}
& \omega_{x}=\frac{\partial w}{\partial y}-\frac{\partial v}{\partial z} \\
& \omega_{y}=\frac{\partial u}{\partial z}-\frac{\partial w}{\partial x}=\frac{1}{u}\left(v \cdot \omega_{x}+\frac{1}{\rho} \frac{\partial P_{0}}{\partial z}\right)
\end{aligned}
$$

and where $\overline{\bar{\beta}}_{2}$ is the overall area-averaged outlet flow angle expressed as:

$$
\overline{\overline{\beta_{2}}}=\tan ^{-1}\left(\begin{array}{l}
\overline{v_{2}} / \bar{z} \\
\bar{u}_{2}
\end{array}\right)
$$

$\bar{u}_{2}$ and $\bar{v}_{2}$ are the area-average axial and pitchwise velocities at the outlet measurement plane (MacIsaac et al., 2010). Equation (3.22) is an approximation used to extract the vorticity from a single axial measurement plane and are derived from the incompressible Euler equations (Gregory-Smith et al., 1988; Yaras and Sjolander, 1990). Yaras and Sjolander (1990) studied its validity in viscous flows and concluded that the error 
in vorticity was very small everywhere except in the vortex core where the velocity gradient is changing most rapidly and the viscous effects are most important. However, it was found that the errors were small enough to use equation (3.22). The non-dimensional streamwise vorticity coefficient is given as:

$$
C_{\omega_{s}}=\frac{\omega_{s} B_{x}}{V_{C L, 1}}
$$

where $B_{x}$ and $V_{C L, 1}$ are the axial chord of the blade and the inlet centerline velocity, respectively.

Another indicator of the strength of the secondary flow is the secondary kinetic energy, which is the kinetic energy associated with the secondary velocity vectors in a plane perpendicular to the area-averaged outlet flow angle, $\overline{\bar{\beta}}_{2}$. It is defined as:

$$
S K E=\frac{1}{2} \rho\left(v_{\mathrm{sec}}^{2}+w_{\mathrm{sec}}^{2}\right)
$$

where $v_{\text {sec }}=-u_{2} \sin \overline{\overline{\beta_{2}}}+v_{2} \cos \overline{\overline{\beta_{2}}}$ and $w_{\text {sec }}=w_{2}$. The non-dimensional secondary kinetic energy, $C_{S K E}$, is expressed as:

$$
C_{S K E}=\frac{v_{\mathrm{sec}}^{2}+w_{\mathrm{sec}}^{2}}{V_{C L, 1}^{2}}
$$

The secondary kinetic energy downstream of the test section will eventually dissipate as irrecoverable total pressure loss with sufficient mixing (MacIsaac et al., 2010). Thus, lower values of secondary kinetic energy at a given measurement plane would result in lower mixed-out losses, and therefore represents a favourable characteristic.

\subsubsection{Axial Velocity Ratio (AVR)}

An approximate measure for the streamtube convergence or divergence at midspan is the axial velocity ratio (AVR). For a low-speed linear cascade where the flow is incompressible (i.e. density term can be omitted), the axial velocity ratio is defined as:

$$
A V R=\frac{u_{2, \text { midspan }}^{\prime}}{u_{1, \text { midspan }}^{\prime}}
$$


where $u_{1, \text { midspan }}^{\prime}$ and $u_{2, \text { midspan }}^{\prime}$ are the mass-averaged axial velocity at midspan upstream and downstream of the cascade, respectively. In order to have two-dimensional flow at midspan of the cascade, AVR should be equal to 1.0. This is a necessary but not sufficient condition. Convergence in the flow in the spanwise direction would result in an AVR greater than 1.0, whereas flow divergence in the spanwise direction would result in AVR less than 1.0.

\subsubsection{Oil Surface Flow Visualisation}

Oil surface flow visualisations were conducted on the blade suction and pressure surfaces and also on the flat and contoured endwalls of the test section. The mixture used for the visualisations was made of 10W-30 motor oil and fluorescent red powder pigment, called Day-Glo AX-13-5 Rocket Red (Abo El Ella and Sjolander, 2012). The final mixture was found after several iterations to find the proper viscosity that would work in a compressor cascade where the flow decelerates.

The flow visualisation techniques were developed based on the work of previous investigators (Taremi and Sjolander, 2011; Abo El Ella and Sjolander, 2012; Knezevici, 2011) and involved applying a thin coat of the oil/powder mixture on the blade surfaces using a paint brush. Painting techniques were developed to avoid brush marks that would obscure the flow streamlines and also to reduce pooling. Once the painted blade surface was ready, it was installed in the test section and the wind tunnel was operated at the design condition. At the end of the test, the blade was removed from the test section and installed in a darkened tent created by the author where it was illuminated with ultraviolet lighting. The tent was necessary to largely eliminate the visible light since the sensor in digital cameras is only weakly sensitive to ultra-violet light.

Photos were taken using a mid-range camera (Canon PowerShot SX120 IS) with a resolution of $3648 \times 2736$ pixels and manual focus. The camera was installed on a tripod to ensure a stable and focused image capture. The typical exposure time for one photo was ten seconds.

A similar technique was used for the endwall flow visualisation but the darkened tent was built around the test section to avoid removing the endwall and to allow photos to be 
taken before the flow features were disturbed by the mixture moving on the vertical surface due to gravity.

Post-processing of pictures was done using Serif PhotoPlus Starter software to remove the remaining visible purple-blue light on the image and to improve the quality. The enhanced photos were imported into Microsoft Visio 2007 to allow scales to be applied to the images and thus allow the locations of features to be determined quantitatively. 


\section{Chapter 4 Results and Discussions}

\section{Secondary Flows at Design Incidence}

\subsection{Introduction}

This chapter presents the results of the investigations on the flow field of a linear compressor cascade. This study is the first experimental investigation of the CEGV-1 airfoil in the low speed wind tunnel set-up described in Chapter 3. The testing was conducted at design incidence and at an inlet Reynolds number of 150,000 based on the axial chord and the inlet centerline velocity. The current chapter examines the secondary flows of the compressor cascade with and without the implementation of endwall contouring. The objective is to have a better understanding of the secondary flows within the blade passage and downstream of the trailing edge and their effect on loss generation. The cascade was first investigated with a flat endwall. Following this investigation, the cascade was then tested with the application of non-axisymmetric endwall contouring.

Section 4.2 provides the experimental results for both planar and contoured endwalls. The inlet flow, the blade loading distributions, the flow visualisation and the pressure probe results are provided in the separate sub-sections. Finally, the last section summarizes the findings and conclusions. 


\subsection{Experimental Results}

\subsubsection{Inlet Conditions}

As discussed in Section 3.9.2, the turbulence generating grid used in the present experiment generated extra total pressure loss near midspan, resulting in a non-uniform inlet total pressure distribution. The non-uniformity of the total pressure is shown on Figure 4.1. The boundary layer was traversed from the endwall to midspan using a Pitot probe located 1.2 axial chords upstream of the leading edge of the cascade at mid-passage. To account for the pitchwise non-uniformities in the inlet flow, the inlet plane was traversed using a threehole pressure probe over one blade pitch from the endwall to half span. The resulting total pressure distribution was mass-averaged to obtain the reference inlet total pressure coefficient $\left(C_{P 0,1}^{\prime \prime}\right)$ used to calculate the total-pressure loss coefficient $\left(\Omega_{\text {total }}\right)$. The inlet endwall boundary layer parameters and mass-averaged pressure coefficients are given in Table 4.1. The displacement thickness $\left(\delta^{*}\right)$ and the momentum thickness $(\theta)$ were obtained by integrating the inlet boundary layer from the endwall to the point of maximum velocity, which in this case, is approximately at $\mathrm{z} / \mathrm{h}=0.2$. The boundary layer shape factors $(\mathrm{H})$ for both endwall configurations are typical of turbulent boundary layers with zero pressure gradient.

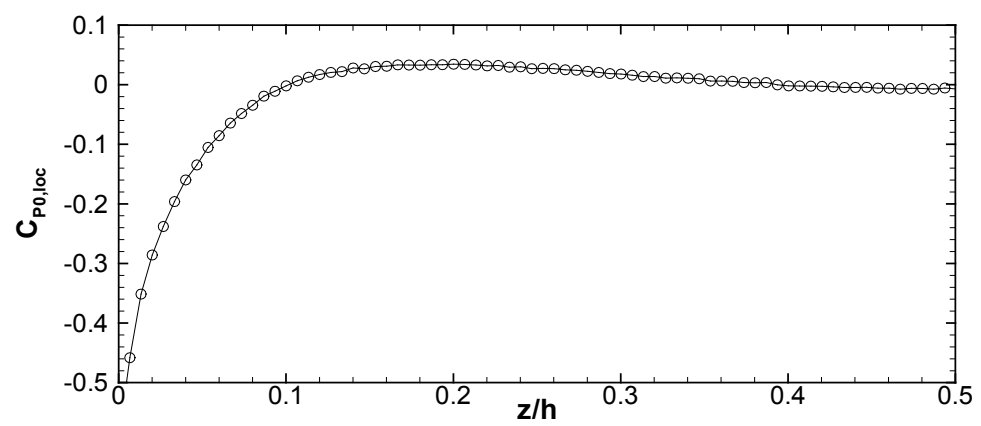

Figure 4.1: Spanwise variation of total pressure coefficient at design incidence measured with a Pitot probe 1.2Bx upstream of the leading edge and $y / s=0.5$ 
Table 4.1: Summary of experimental results at design incidence measured $1.2 B_{x}$ upstream of the blade leading edge.

\begin{tabular}{cccc} 
LOCATION & $\begin{array}{c}\text { FLOW } \\
\text { QUANTITY }\end{array}$ & FLAT & CONTOURED \\
\hline INLET & $\delta / \mathrm{h}$ & 0.118 & 0.118 \\
& $\delta^{*}[\mathrm{~mm}]$ & 2.57 & 2.25 \\
& $\theta[\mathrm{mm}]$ & 1.99 & 1.80 \\
$\mathrm{H}$ & 1.29 & 1.25 \\
& $\mathrm{C}_{\mathrm{q}, 1}{ }^{\prime \prime}$ & 0.982 & 0.987 \\
& $\mathrm{C}_{\mathrm{q}, 1 \text { midspan }}{ }^{\prime}$ & 0.995 & 0.998 \\
& $\mathrm{C}_{\mathrm{P} 0,1^{\prime \prime}}$ & -0.011 & -0.010
\end{tabular}

The differences observed between the two endwall boundary layer parameters are mainly an indication of scatter. However, different potential effects from the blade and endwall configurations, different flow control surfaces and blockage configurations, and small differences in the endwall boundary layer bleed flows upstream of the cascade could also be sources of scatter observed between the two endwall configurations. The differences in mass-averaged dynamic and total pressure coefficients observed between both enwalls are within probe measurement error.

\subsubsection{Blade Loading Distributions}

The size and strength of the secondary flows are known to be influenced by the overall blade loading and its distribution (Zoric et al., 2007a; Benner et al., 2004; Sonoda et al., 2008; Dickens and Day, 2009). The pressure distributions for CEGV-1 are shown at three different spanwise locations on Figure 4.2 (a), (b) and (c). The blade loadings are plotted as the static pressure coefficient $\left(C_{P S, b l}\right)$ versus the fraction of axial chord $\left(x / B_{x}\right)$ and are shown for both flat and contoured endwalls test cases. The blade loading static pressure coefficient is defined as

$$
C_{P S, b l}=\frac{P_{0, C L, 1}-P_{l o c}}{q_{C L, 1}}
$$


a) Midspan

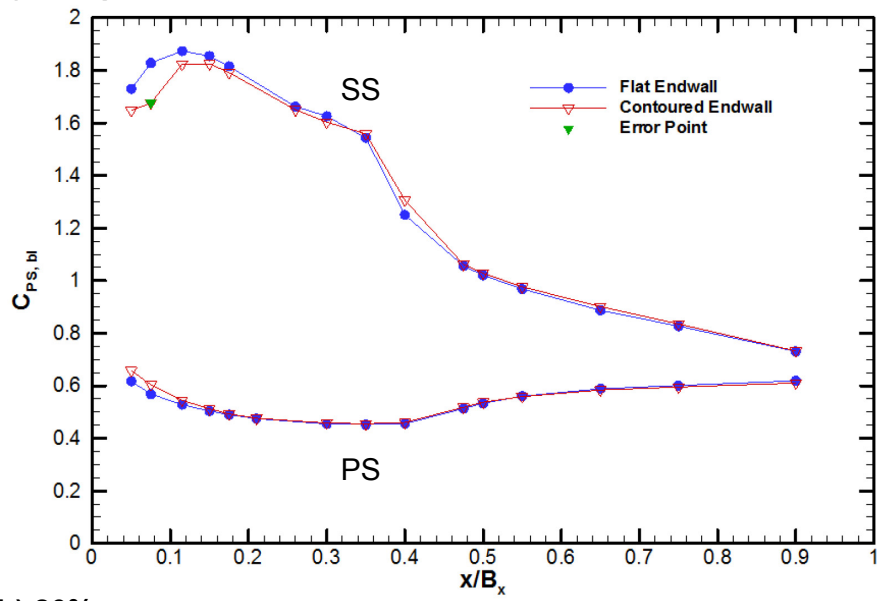

b) $\mathbf{2 0 \%}$ span

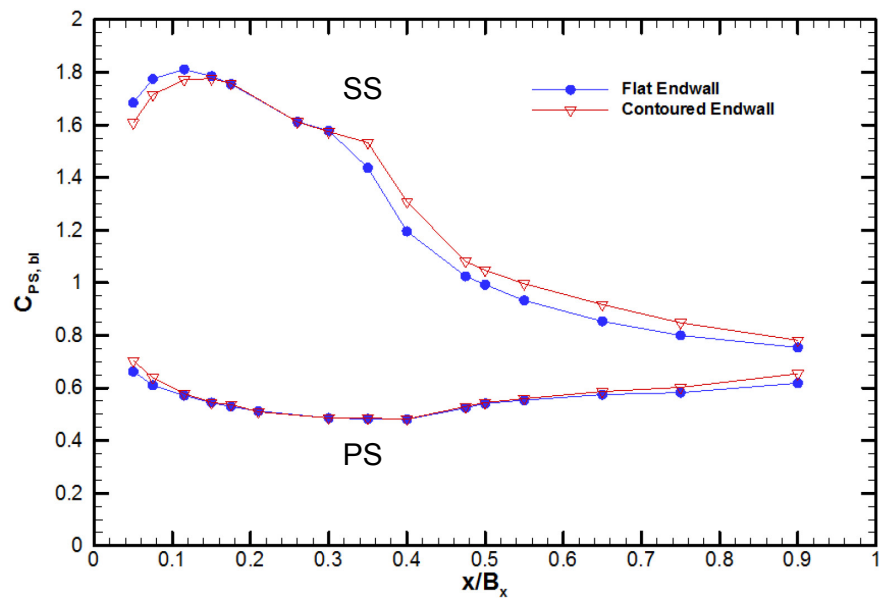

c) $\mathbf{5 \%}$ span

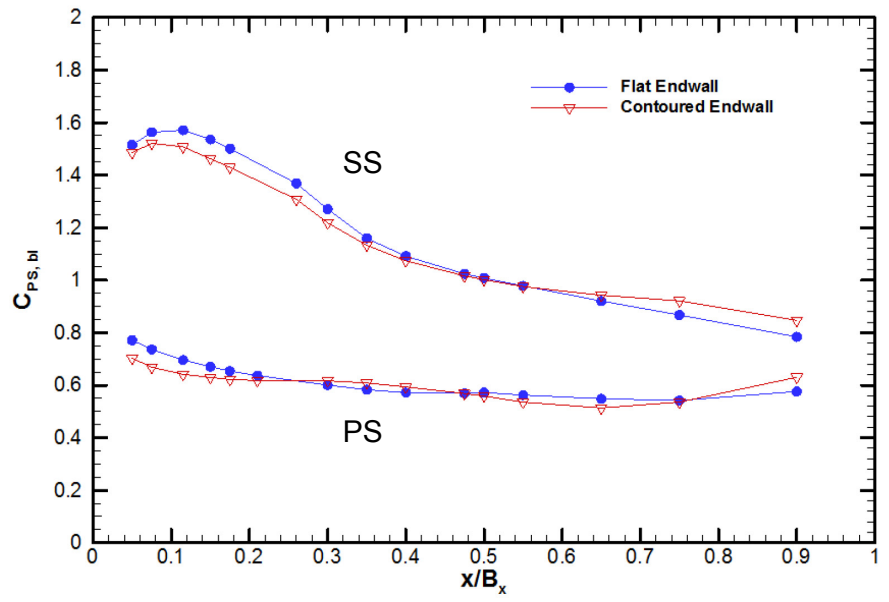

Figure 4.2: Blade surface static pressure distribution at $\mathrm{z} / \mathrm{h}=0.50$ (a), $\mathrm{z} / \mathrm{h}=0.20$ (b) and $\mathrm{z} / \mathrm{h}=0.5$ (c) at design incidence 
CEGV-1 airfoil is considered as a front-loaded airfoil with a suction peak located at approximately $x / B_{x}=0.12$. This means that the resultant tangential force is acting towards the front part of the airfoil. From the loadings at midspan (Figure 4.2 (a)), it can be observed that the suction surface (SS) boundary layer sees a rapid acceleration from the leading edge of the blade to the suction peak. At this point, the boundary layer decelerates gradually towards the trailing edge. From Figure 4.2 (a), the blade appears to experience a small separation bubble on its suction surface. Separation bubbles can be identified by a plateau in the local pressure distribution. The flow separates approximately at $x / B_{x}=0.25$ and reattaches around $x / B_{x}=0.48$. Flow visualisation, which will be presented later, verifies that the observed plateau in the static pressure distribution is a region of two-dimensional separation.

It can be observed from Figure 4.2 (a) that the static pressure distributions of the flat and contoured endwalls test cases agree for most of the blade passage at midspan. The blade loading static pressure coefficient at the trailing edge is approximately equivalent to the outlet dynamic pressure. For both flat and contoured endwalls, the coefficients are approximately the same at the trailing edge, meaning that the blades are turning and decelerating the flow in a similar manner. Thus, it can be concluded that the application of endwall contouring has little effect on the flow field towards midspan.

However, some differences are observable near the leading edge of the blade between the two cases. The loading appears to be slightly higher in the first $20 \%$ axial chord for the flat endwall. The differences in loadings near the leading edge are likely due to small differences in the flow incidence at the leading edge. The pitchwise inlet flow angle, shown on Figure 4.3, seems to confirm the previous statement. Both flat and contoured endwall cases differ slightly from the intended design inlet flow angle. As observed for the flat endwall, the inlet flow angle at midspan has a slight positive incidence with respect to the design incidence. This increased incidence could explain the higher loading at midspan near the leading edge of the blade for the flat endwall test. The deviations are in the opposite direction for both cases, but are within the defined tolerance for the flow angle measurements with the three-hole probe $\left(+/-0.5^{\circ}\right)$. 


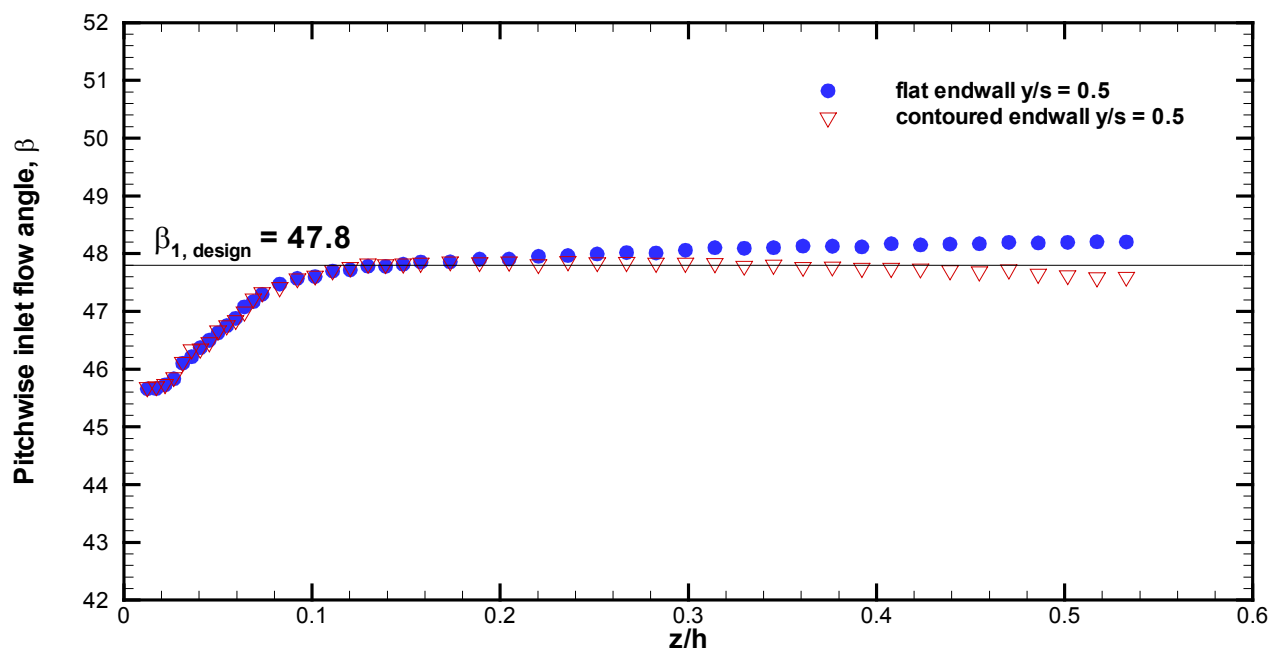

Figure 4.3: Pitchwise inlet flow angle deviation from the design inlet angle $\beta_{1, d e s i g n}$ measured at $1.2 B x$ upstream of the blade leading edge and at $y / s=0.5$

From Figure 4.2 (b), the loadings at $20 \%$ span are slightly lower than at midspan. As observed, the suction surface static pressures are slightly higher than at midspan, while the pressure surface static pressures are approximately the same. Unlike at midspan, the endwall contouring appears to influence the suction surface boundary layer downstream of the separation bubble. At this spanwise location with contouring applied, the boundary layer is not decelerated as much as for the flat endwall case, suggesting a slight reduction in diffusion. As it will be later discussed in Sections 4.2.3 and 4.2.4, the endwall contouring alters the cross-passage pressure gradient to enhance the cross-flow and modify its interaction with the suction surface boundary layer, therefore affecting the loading on the blade.

Figure 4.2 (c) shows the blade loading at 5\% span. As seen, the loadings are lower than at midspan and at $20 \%$ span, which is potentially due to the positive bow on the suction surface locally increasing the local static pressures. It is worth noting that the separation bubble observed at 50 and $20 \%$ spans is not present at $5 \%$ span. This suggests that the streamwise flow in the suction surface boundary layer is affected by its interaction with the endwall cross-flow moving up the blade surface, such that to prevent the two-dimensional separation. However, moving towards the trailing edge, the loadings are slightly larger than for the other spanwise positions in the aft part of the passage, suggesting that the corner stall 
has started to develop. The corner stall influences the boundary layer and static pressures on the suction surface of the blade near the endwall, therefore affecting the loading at $5 \%$ span, as it will be discussed in Section 4.2.3. Additionally, like at $20 \%$ span, the application of endwall contouring appears to affect the loadings at 5\% span. The application of endwall contouring increases the local static pressures near the suction surface of the blade. These higher static pressures on the endwall create a spanwise pressure gradient that drives the endwall flow onto the suction surface, therefore modifying its interaction with the suction surface boundary. This characteristics appears to alter the loadings as it can be observed from the higher static pressures (lower $C_{P S, b l}$ ) in the front section of the blade for the contoured endwall (Figure 4.2 (c)).

In general, it appears that, for the CEGV-1 airfoil, the overall blade loading decreases approaching the endwall. This characteristic is presumably due to the corner stall, to the lower dynamic pressure in the endwall boundary layer as well as to the interaction between the endwall flow and suction surface boundary layer.

\subsubsection{Flow Visualisation Results}

The surface flow visualisation studies were conducted at design incidence and at the design Reynolds number using a fluorescent-dye/oil mixture described in Section 3.9.5. The nomenclature used for the interpretation is based on Sieverding (1985) and adapted for a compressor cascade. This nomenclature was used by several other Carleton investigators such as Benner (2003), Knezevici (2011) and Taremi et al. (2010). Dashed lines are used for flow separation, dotted lines for flow reattachment and arrows for the surface shear stress vectors.

Figure 4.4 shows endwall and suction surface oil surface flow visualisations overlaid with interpreted separation streamlines for the flat and contoured endwall configurations. On the flat endwall (Figure 4.4 (a)), the incoming endwall boundary layer fluid meets the primary separation line $S_{1}$ at the saddle point $A_{1}$. The primary separation line $S_{1}$ represents the separation of the inlet boundary layer from the endwall due to the adverse pressure gradient created by the blade stagnation point. The secondary separation line $\mathrm{S}_{2}$ represents the liftoff line of the so-called horseshoe vortex and is located just upstream of the blade 
leading edge. At this point, the horseshoe vortex is wrapped around the leading edge of the blade, as schematically shown on Figure 2.4 (a). The subscripts "p" and "s" are used to define the pressure and suction side legs of the separation lines.

a) Flat Endwall

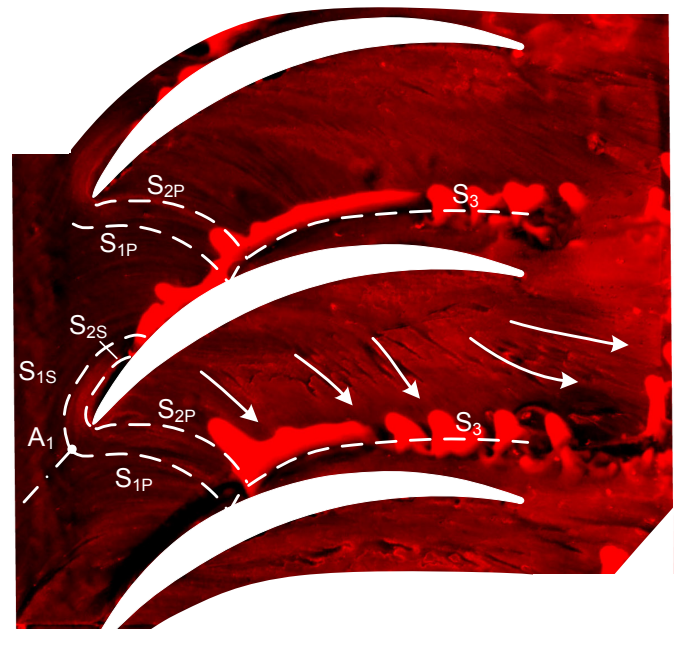

b) Flat Suction Surface

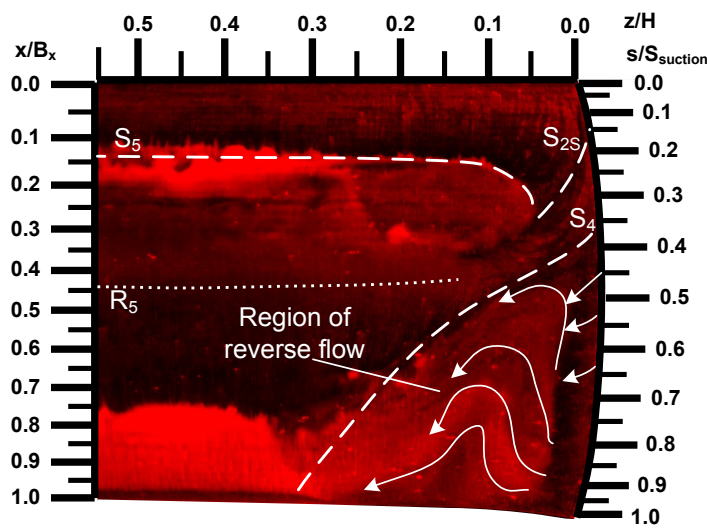

c) Contoured Endwall

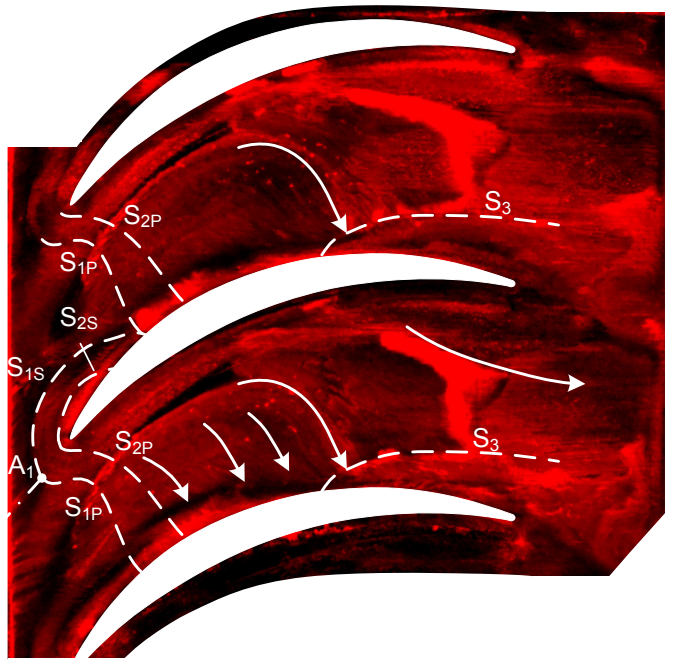

d) Contoured Suction Surface

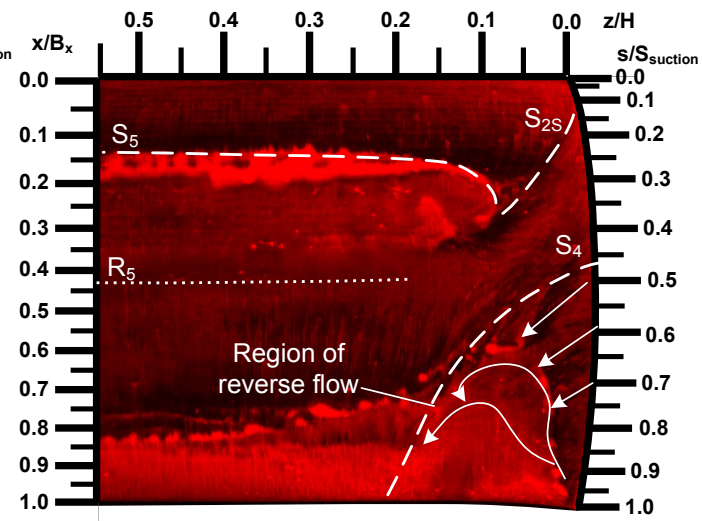

Figure 4.4: Endwall and suction surface oil surface flow visualisations for the flat (a, b) and contoured (c, d) endwall configurations at design incidence

The incoming inlet boundary layer fluid is funnelled between the suction side leg of the horseshoe vortex $S_{2 S}$ and the pressure side leg of the horseshoe vortex $S_{2 P}$ of the adjacent blade. This fluid is swept towards the blade suction surface at which point it moves away from the endwall up the suction surface. The "funnelled region" is a region of high shear stress, which is indicated by the smaller amount of pigment. As observed near the suction 
surface of the bottom blade on Figure 4.4 (a), the low amount of pigment suggests that all the funnelled boundary layer fluid was moved up the blade surface. A similar observation can be made for the middle blade, although some of the oil pigment did not moved up the blade surface and accumulated at the intersection of the endwall and the suction surface. It is worth noting that, between the time the wind tunnel was stopped and the time the pictures were taken, drips of oil formed as a result of gravity.

Under the influence of the cross-passage pressure gradient, the pressure legs $S_{1 P}$ and $\mathrm{S}_{2 \mathrm{P}}$ of the horseshoe vortex migrate across the passage towards the adjacent suction surface of the blade. In this process, the pressure side leg $\mathrm{S}_{2 \mathrm{P}}$ entrains low momentum boundary layer fluid and becomes the passage vortex. As seen on Figure 4.4 (a), the passage vortex encounters the suction surface of the blade at approximately $0.35 \mathrm{~B}_{\mathrm{x}}$, where it moves away from the endwall towards half span as indicated by $\mathrm{S}_{4}$ in Figure 4.4 (b).

The suction leg of the horseshoe vortex, $\mathrm{S}_{2 \mathrm{~S}}$, is also influenced by the cross-passage pressure gradient and is directed towards the suction surface. $\mathrm{S}_{2 \mathrm{~S}}$ encounters the suction surface at approximately $0.15 \mathrm{~B}_{\mathrm{x}}$ at which point it migrates off the endwall on the blade surface.

As observed on Figure 4.4 (a), a corner separation line, marked by $\mathrm{S}_{3}$, is formed where the suction surface meets the endwall at approximately $0.35 \mathrm{~B}_{\mathrm{x}}$. It is represented by the accumulation of oil on the endwall. The corner separation $S_{3}$ starts near the suction surface and moves away from the surface to about $25 \%$ of the blade passage. The growth of the corner separation can be seen as the distance between $S_{3}$ and the suction surface increases (Hodson and Dominy, 1987). The region enclosed by $\mathrm{S}_{3}$ and the blade surface is a region of low surface shear stress. The low momentum fluid in this region is further decelerated by the overall adverse pressure gradient existing in the test section, potentially feeding the corner separation. The larger the angle of incidence of $S_{2 P}$ makes with the corner separation line $S_{3}$, the more intensely the corner vortex is strengthened (Knezevici, 2011). The new endwall boundary layer forming downstream of $\mathrm{S}_{1 \mathrm{P}}$, shown by the arrows on Figure 4.4 (a), is swept towards the suction surface of the blade by the cross-passage pressure gradient, which also results in strengthening the existing the corner vortex. 
The suction surface flow visualisation is shown on Figure 4.4 (b) with scales marking the approximate fraction of axial chord $\mathrm{x} / \mathrm{B}_{\mathrm{x}}$ on the left, of suction surface length $\mathrm{s} / \mathrm{S}_{\text {suction }}$ on the right and of span $\mathrm{z} / \mathrm{H}$ at the top. Figure 4.4 (b) shows three dominant separation lines and one reattachment line. $S_{5}$ and $R_{5}$ mark the separation and reattachment of the approximately two-dimensional suction surface boundary layer. This corresponds to the separation bubble noted on the blade loadings (see Figure 4.2). From the blade loadings, it was estimated that separation occurred approximately at $0.25 \mathrm{~B}_{\mathrm{x}}$, whereas, it appears to occur between 0.15 $0.17 \mathrm{~B}_{\mathrm{x}}$ from the flow visualisation. As discussed in the previous section, suction surface separation results in additional profile losses to those generated from viscous dissipation in the boundary layer (Taremi et al., 2010). The region downstream of $\mathrm{R}_{5}$ is a region of high shear stresses in which the boundary layer is turbulent. The separation line $\mathrm{S}_{4}$ is usually associated with the passage vortex and is considered as a three-dimensional separation. It starts at the endwall corner at approximately $0.3 \mathrm{~B}_{\mathrm{x}}$ and grows towards the trailing edge to about $30 \%$ span. The region enclosed between $\mathrm{S}_{4}$ and the endwall shows intensive cross-flow from the passage vortex along with regions of reverse flow as seen on Figure 4.4 (b). This region corresponds to the corner stall and is a significant source of losses. This separated region might be the source of the higher loadings observed towards the trailing edge of the blade at $5 \%$ span on Figure 4.2 (c).

The separation line $\mathrm{S}_{2 \mathrm{~S}}$ is the extension of the suction leg of the horseshoe vortex on the blade surface. $\mathrm{S}_{2 \mathrm{~S}}$ initially starts at the endwall and moves along the blade surface until it meets the separated flow downstream of $\mathrm{S}_{5}$. At this point, it seems to separate from the blade surface as the separation line disappears.

The application of endwall contouring does not change the broad physics of the flow. The features observed in Figure 4.4 (a) and (b) are also observed in the contoured flow visualisation shown in Figure 4.4 (c) and (d). The streamline curvatures in a contoured endwall will affect the endwall static pressures. A convex curvature locally accelerates the flow and reduces endwall static pressure. Such a feature can be observed on Figure $3.5\left(\mathrm{H}_{1}\right.$ \& $\mathrm{H}_{2}$ ). A concave curvature locally diffuses the flow, resulting in an increase in local endwall static pressure. This feature can also be observed in Figure $3.5\left(\mathrm{~T}_{1} \& \mathrm{~T}_{2}\right)$. 
Although the broad physics do not change, there are some noticeable differences between the endwall flow visualisations for the flat and the contoured endwalls. As observed on Figure 4.4 (c), the saddle point $A_{1}$ moved slightly upstream compared to the flat endwall case. This is a result of the concave curvature $\left(T_{1}\right)$ occupying most of the passage near the leading edge of the blade. The local diffusion on the endwall also caused an increase in the distance between $S_{1}$ and $S_{2}$ which means that the inlet boundary layer fluid separated on a longer distance before lifting off the endwall surface and forming the horseshoe vortex.

The concave curvature $\left(T_{1}\right)$ in the forward part of the passage diffuses the flow locally, which means that the local static pressure increases resulting in a higher cross-passage pressure gradient. The increase in the cross-passage pressure gradient results in higher momentum fluid being directed towards the suction surface/endwall corner. This action mitigates the corner stall by delaying its formation, thus resulting in a reduction of secondary losses. The increase in the intensity of the cross-flow results in something that Sieverding (1985) calls "strong convergence".

The higher local static pressures on the endwall create a spanwise pressure gradient, which encourages the endwall flow to migrate on the suction surface of the blade. This results in delaying the corner stall which would otherwise occur if the low momentum fluid would remain on the endwall corner (Harvey and Offord, 2008). The reduction in the extent of the corner stall can be observed by looking at the downstream shift of the corner separation $\left(\mathrm{S}_{3}\right)$ starting point, from Figure 4.4 (a) and (c).

The cross-flow for the contoured endwall case is more perpendicular to the suction surface of the blade compared to the flat endwall. These perpendicular lines are a result of the higher pitchwise pressure gradient due to local diffusion. Although the intensity of the crossflow is increased, because of diffusion, the local cross-flow fluid is decelerated and has lower shear stress/lower momentum. This could be observed comparing Figure 4.4 (a) and (c) for which the amount on oil within the cross-passage flow is larger for the contoured case. The shear stress vectors are not as well defined for the contoured configuration compared to the flat one.

Compared to the flat endwall configuration, the separation line $S_{2 s}$ with contouring encounters the suction surface at a location slightly downstream $\left(x / B_{x}=0.20\right)$. The increase 
in axial penetration of $\mathrm{S}_{2 \mathrm{~s}}$ and the low shear stress cross-flow delays the formation of the corner separation $\mathrm{S}_{3}$ as observed on Figure 4.4 (c).

For the contoured endwall case, the corner separation occurs at $\mathrm{x} / \mathrm{B}_{\mathrm{x}}=0.60$, which is approximately $25 \%$ downstream of the flat endwall case. However, towards the trailing edge, because of the convex curvature $\left(\mathrm{H}_{2}\right)$, the local flow is accelerated and, being subject to the cross-passage pressure gradient, appears to intensify the corner separation as seen on Figure $4.4(\mathrm{c})$.

With regards to the suction surface flow visualisation of the contoured endwall, on Figure $4.4(\mathrm{~d})$, it is observed that the penetration height of the three-dimensional separation line $\left(\mathrm{S}_{4}\right)$ is slightly reduced compared to the flat endwall case (Figure 4.4 (b)). Also, the separation line $\left(\mathrm{S}_{4}\right)$ starts at a chordwise position downstream of the location with the flat endwall. The application of endwall contouring appears to affect the cross-passage flow such that the size of the passage vortex is reduced. As it will be shown in the next section, the size of the passage vortex is effectively confined to a smaller area of the passage. Also, as observed on Figure 4.4 (d), the endwall cross-flow migrating on the suction surface appears stronger than for the flat endwall and the region of reverse flow is slightly smaller. This is as expected as one of the objectives of endwall contouring for a compressor cascade is to delay the formation of the corner stall by increasing the strength of the cross-flow and its migration up the suction surface of the blade.

\subsubsection{Downstream Flow Field Measurements}

\subsubsection{Total Pressure Distributions}

Figure 4.5 shows colour flood contour plots of total pressure deficit coefficient for both flat and contoured endwall test cases as measured on a plane located 1.6 axial chords downstream of the blade leading edge.

The results are plotted over 1.2 blade pitches and from the endwall to half span with the appropriate pitch-to-chord ratios (see Table 3.2). The projections of the trailing edges are shown as dashed lines at $\mathrm{y} / \mathrm{s}=0$ and $\mathrm{y} / \mathrm{s}=1$. Here, the high loss regions are represented by red, the low loss regions by blue and the no loss regions by white. 
Both floods show similar general features such as one region of elevated loss near the endwall closer to the suction surface and a two-dimensional wake from approximately $\mathrm{z} / \mathrm{h}=$ 0.3 to midspan. The magnitude of the peak loss is also similar with a value hovering around $C_{P 0}=-0.45$. However, some differences can be observed. For the flat endwall, the elevated region of loss extends from approximately $\mathrm{y} / \mathrm{s}=0.20$ to 0.55 and $\mathrm{z} / \mathrm{h}=0.06$ to 0.26 , with a peak loss located at approximately $\mathrm{y} / \mathrm{s}=0.35$ and $\mathrm{z} / \mathrm{h}=0.14$. For the contoured endwall, the size and strength of the regions of elevated loss are similar to those for the flat endwall case, but the location is changed. The elevated loss region is shifted closer to the suction surface/endwall corner, extending from $\mathrm{y} / \mathrm{s}=0.00$ to 0.45 and $\mathrm{z} / \mathrm{h}=0.02$ to 0.18 with a peak loss at approximately $\mathrm{y} / \mathrm{s}=0.20$ and $\mathrm{z} / \mathrm{h}=0.08$.

The application of endwall contouring results in the suction side leg of the horseshoe vortex moving towards the suction surface of the blade with the objective of delaying the corner stall (Harvey and Offord, 2008). The increased cross-passage pressure gradient on the endwall drives high momentum fluid in the suction corner to reduce the extent of the corner stall. The spanwise pressure gradient created from the positive bow of the suction surface and from the concave curvature in the passage alters the interaction between the endwall flow and the suction surface boundary layer. These actions then result in a concentrated high loss region closer to the suction surface/endwall corner when moving towards the downstream plane. 
a) Flat Endwall

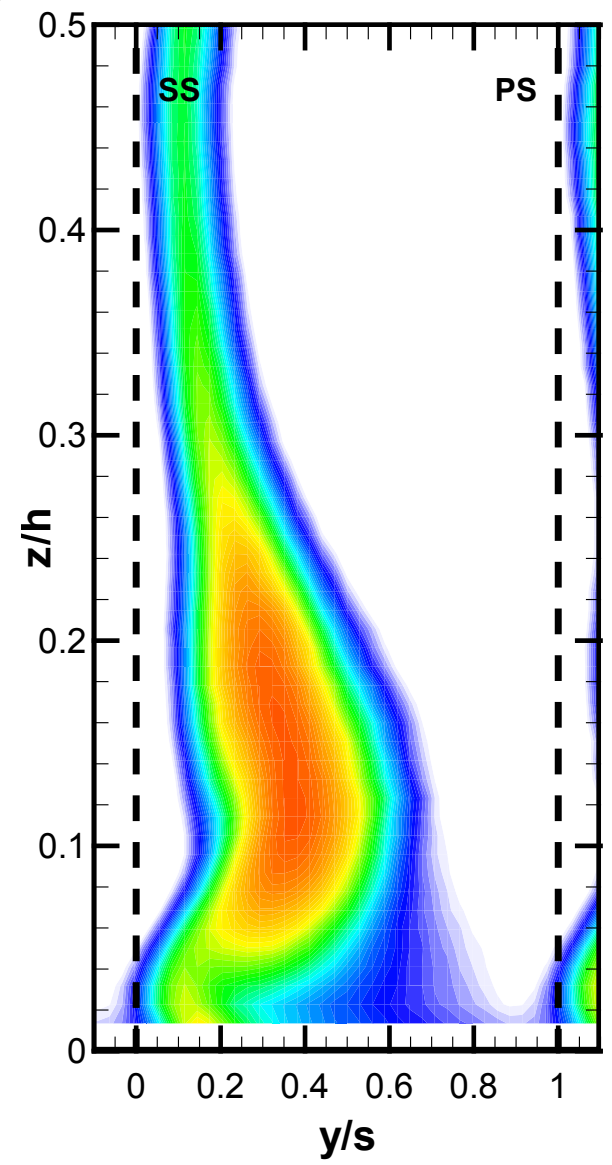

b) Contoured Endwall

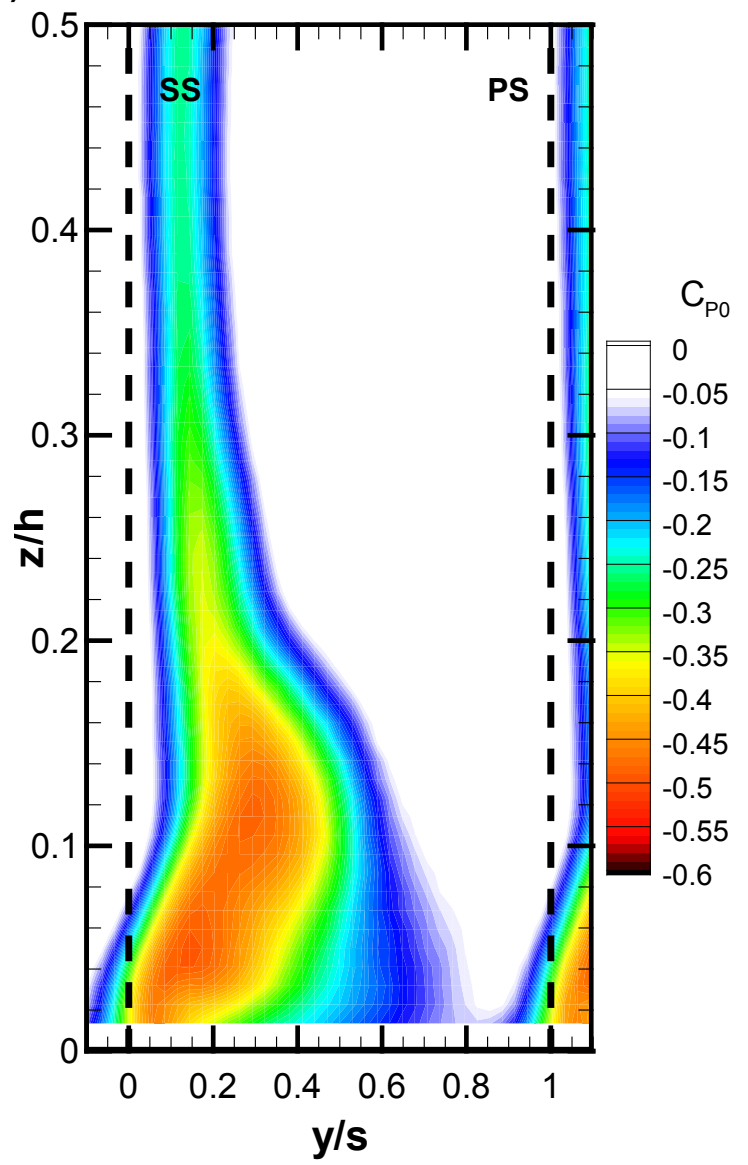

Figure 4.5: Total pressure deficit coefficient $\left(\mathrm{C}_{\mathrm{PO}}\right)$ floods at $1.60 \mathrm{~B}_{\mathrm{x}}$ for the flat $(\mathrm{a})$ and contoured (b) endwall configurations at design incidence

\subsubsection{Streamwise Vorticity Fields}

Figure 4.6 shows the secondary velocity vectors surimposed on a colour flood of streamwise vorticity coefficient. The secondary velocity vectors represent the components of velocity on a plane normal to the primary flow direction. The streamwise vorticity is derived from the incompressible Euler equations as per Gregory-Smith et al. (1988). This approximation is used as it allows all components of the vorticity vector to be estimated from measurements on a single traverse plane. The validity of this approximation in viscous flows has been studied by Yaras and Sjolander (1990) and it was concluded that the error in vorticity was very small everywhere except in the vortex core where the velocity gradient is 
changing most rapidly and the viscous effects are most important. However, even in this region, it is considered that the losses are small enough to use this assumption.

The streamwise vorticity represents the local rotation of the fluid relative to the streamwise direction. It is used to identify the different vortical structures and to quantify their strength and sense of rotation (Knezevici, 2011). The strength is obtained here from the magnitude of the streamwise vorticity coefficient, defined in Equation (3.24) in Section 3.9.4.2. The streamwise vorticity is defined using the right hand rule, such that yellow and red regions indicate a negative rotation about the streamwise axis, while the green and blue regions represent a positive rotation.

a) Flat Endwall

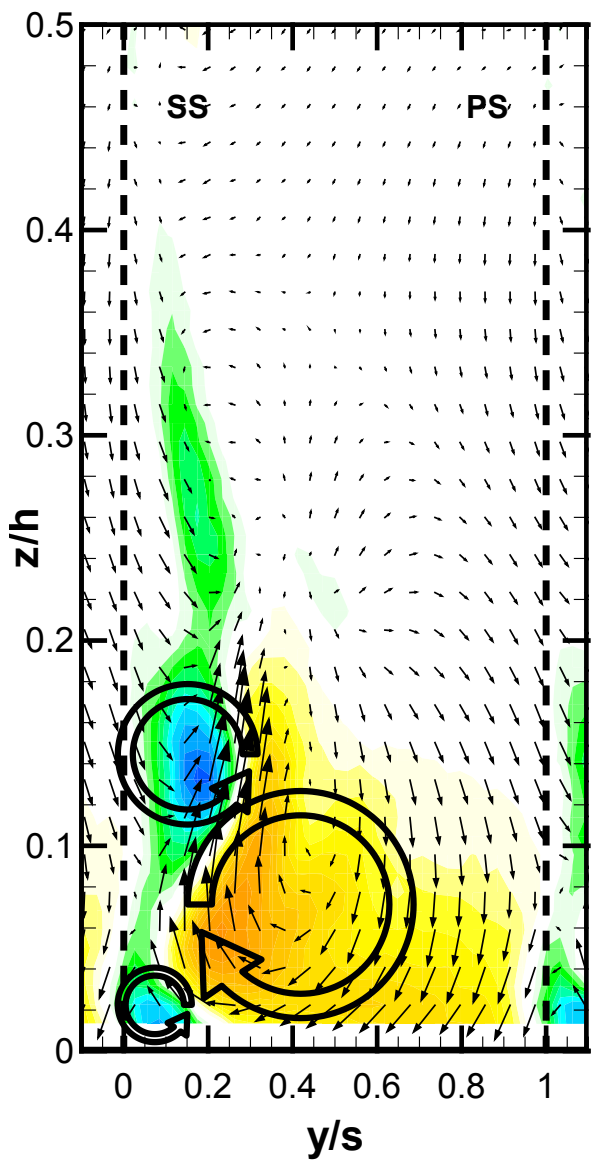

b) Contoured Endwall

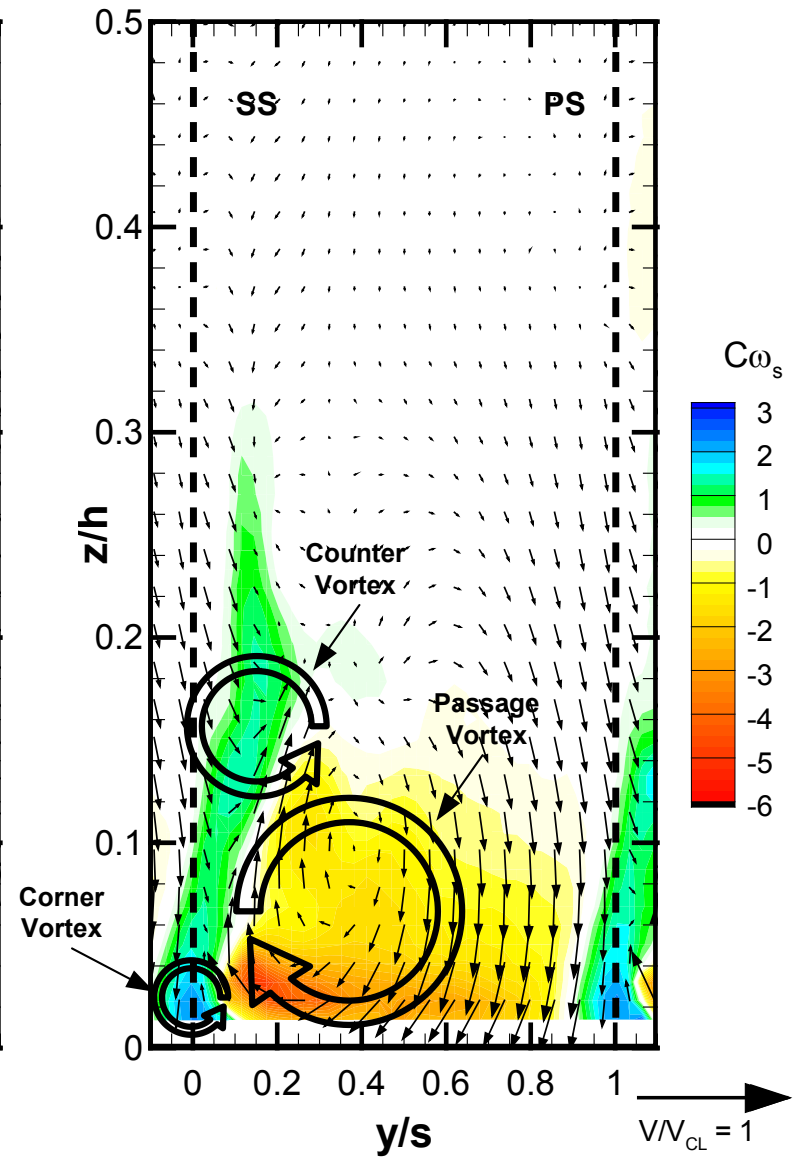

Figure 4.6: Streamwise vorticity coefficient $\left(\mathrm{C} \omega_{s}\right)$ floods overlaid with secondary velocity vectors for the flat (a) and contoured (b) endwall configurations at design incidence 
Three main vortical structures can be identified on Figure 4.6: the passage vortex, the counter vortex and the corner vortex. The passage vortex has negative vorticity about the streamwise direction. The counter vortex and the corner vortex both have positive vorticity. In general, the flow fields for both endwall configurations appear similar, but several features are worth discussing.

The passage vortex originates from the inlet endwall boundary layer. As the horseshoe vortex separates upstream of the blade leading edge, the pressure side of this vortex moves towards the suction surface of the adjacent blade. As the vortex moves across the passage, it entrains the new boundary layer forming downstream of the separation line $S_{2}$ (see Figure 4.4) to form the passage vortex. The peak negative vorticity associated with the passage vortex for the contoured endwall case is stronger closer to the endwall corner compared to the flat endwall. The peak is located at approximately $\mathrm{y} / \mathrm{s}=0.2$ and $\mathrm{z} / \mathrm{h}=0.03$ compared to $\mathrm{y} / \mathrm{s}=0.26$ and $\mathrm{z} / \mathrm{h}=0.07$ for the flat endwall. However, the extent of the passage vortex is slightly smaller for the contoured endwall. As observed, the secondary velocity vectors associated with the passage vortex for the contoured endwall case are stronger than the flat endwall near the pressure side. This is caused by the contoured endwall manipulating the cross-passage flows, which results in the loss regions being closer to the endwall and in a reduction of the mixing with the inviscid core. The local acceleration of the secondary velocity vectors can be explained by the convex endwall curvatures $\mathrm{H}_{1}$ and $\mathrm{H}_{2}$ discussed previously. This result can be observed on Figure 4.5 (b) where the loss core for the contoured endwall is located closer to the suction surface/endwall corner. It is also worth noting that the point at which the secondary velocity vectors revolve does not coincide with the peak magnitude of the passage vortex. Although the strength of the passage vortex, measured here as the peak streamwise vorticity, is larger than for the flat endwall, it can be said that the contouring reduces the overall size of the passage vortex.

The second main vortex present is the counter vortex, which is made up of fluid from the suction leg of the horseshoe vortex and from the endwall boundary layer migrating up the blade surface together with some blade surface boundary layer fluid involved in the separation of the suction surface. The size and strength of this vortex are greatly reduced by the application of endwall contouring. The objective is to delay the corner stall formation and 
the vortex is seen to form further in the passage as evident from the blade separation line $\mathrm{S}_{4}$ on Figure 4.4 (b) and (d). The delayed formation of the corner stall is accompanied by a reduction of the size, magnitude and strength of the counter vortex.

The last vortical structure can be observed near the endwall and to the right of the trailing edge projection. The core of the corner vortex is located at approximately $\mathrm{y} / \mathrm{s}=0.1$ and $\mathrm{z} / \mathrm{h}=0.02$ for the flat endwall case and $\mathrm{y} / \mathrm{s}=0.0$ and $\mathrm{z} / \mathrm{h}=0.02$ for the contoured endwall case. The increased strength of the passage vortex near the endwall for the contoured endwall confines the corner vortex to a slightly smaller area than for the flat endwall. As discussed in Section 4.2.3, the stronger cross-flow of the contouring results in a stronger corner vortex than for the flat endwall case as observed on Figure 4.6 where the streamwise vorticity coefficient in the corner vortex region is higher for the contoured endwall.

The interaction between the passage vortex and the counter vortex for the flat endwall results in the accumulation of high loss fluid between the two vortices. The rotation of these two vortical structures results in high secondary velocity vectors, which tends to sweep the endwall fluid into the inviscid core, increasing mixing and losses with downstream distance. This region of high secondary velocity corresponds to the loss core observed on Figure 4.5. The interaction between the two vortical structures is weaker for the contoured endwall. As observed on Figure 4.6 (b), the counter vortex is weaker than for the flat endwall and the secondary velocity vectors are also smaller, reducing the amount of fluid funneled in the inviscid core. The weaker interaction between the passage and the counter vortices results in lower mass-averaged total pressure loss coefficient and lower mixing losses, as it will be discussed in Section 4.2.4.4.

\subsubsection{Secondary Kinetic Energy}

The secondary kinetic energy (SKE), defined in Section 3.9.4.2, is the kinetic energy associated with the secondary velocity vectors in a plane perpendicular to the area-averaged

outlet flow angle, $\overline{\bar{\beta}}_{2}$. It is an indication of the magnitude of the secondary velocity vectors and of the strength of the cross-flow near the endwall. The secondary kinetic energy is ultimately a source of loss. The losses are generated through mixing as the flow moves downstream. Figure 4.7 shows contour lines of total pressure deficit coefficient overlaid with 
contour floods of the secondary kinetic energy for the flat (a) and contoured (b) endwall configurations.

a) Flat Endwall

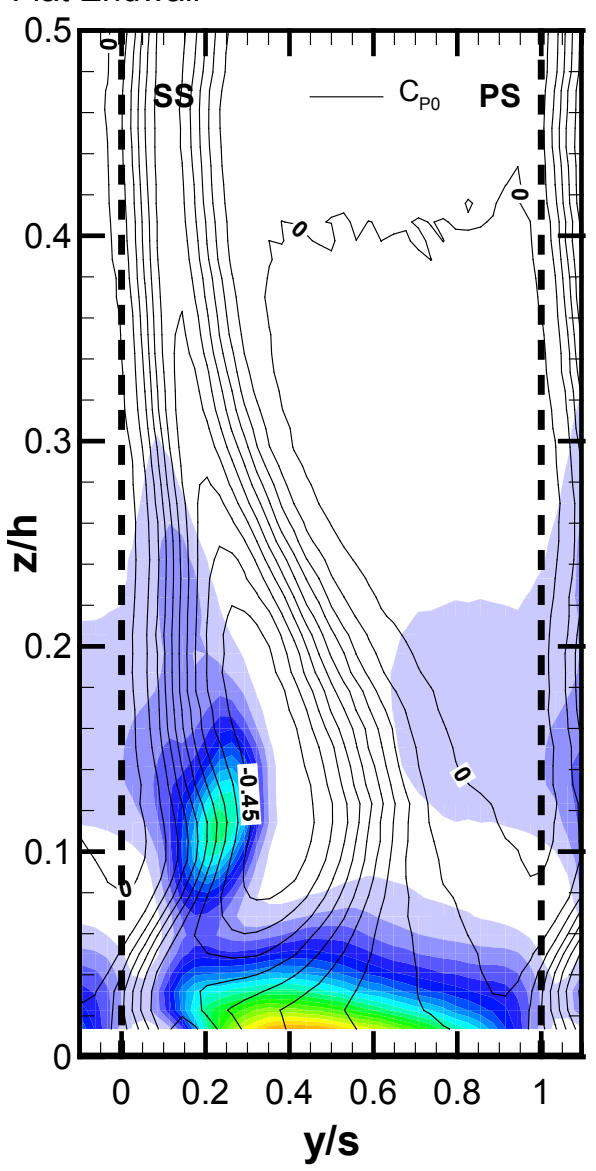

b) Contoured Endwall

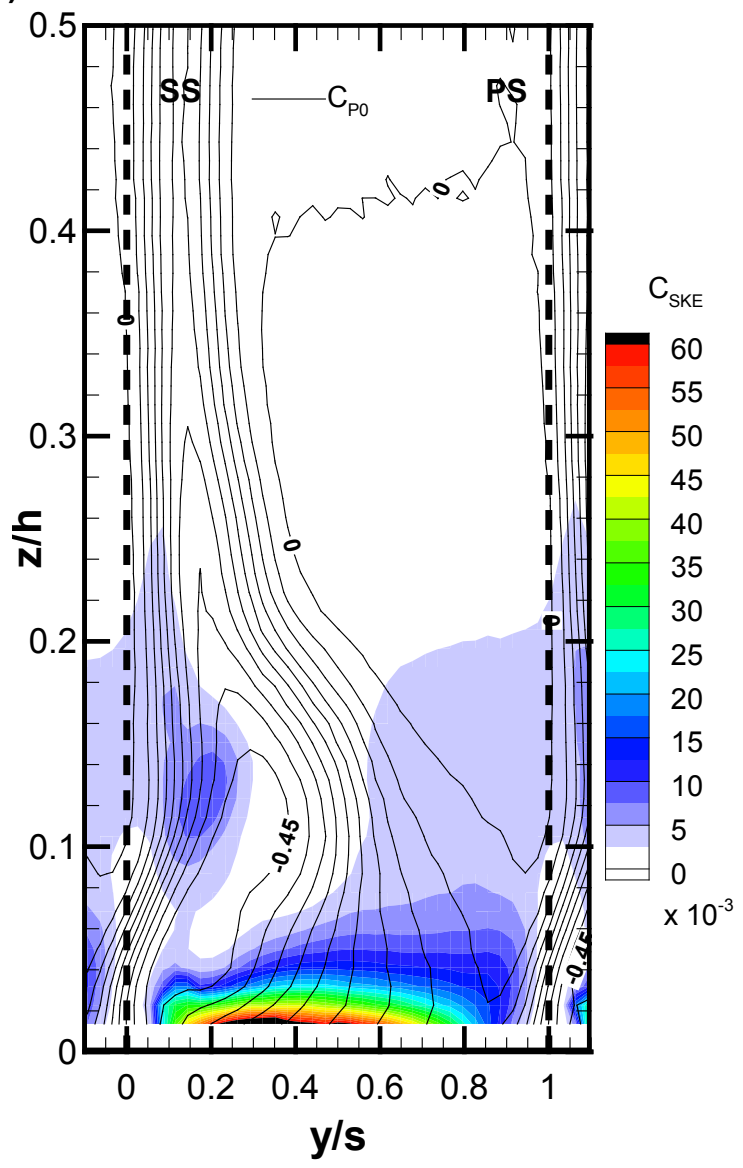

Figure 4.7: Line contours of total pressure deficit coefficient superimposed on flood contours of secondary kinetic energy for the flat (a) and contoured (b) endwall configurations at design incidence

As seen, there are two regions of fluid with relatively high secondary kinetic energy. The first region is located immediately adjacent to the endwall and corresponds to the flow overturning due to the cross-passage pressure gradient. For both endwall configurations, it occupies the majority of the passage near the endwall. The second region corresponds to the fluid moving off the endwall along the blade suction surface in the spanwise direction. This region is located between the passage and the counter vortices identified in the previous 
section. The secondary kinetic energy core is located at approximately $\mathrm{y} / \mathrm{s}=0.25$ and $\mathrm{z} / \mathrm{h}=$ 0.12 for both configurations.

It is evident that, near the endwall, the peak level of secondary kinetic energy is higher for the contoured endwall than for the flat endwall: the peak for the contoured endwall is approximately 1.20 times greater than the peak for the flat endwall. The higher secondary kinetic energy is directly due to the magnitude and strength of the vortical structures, defined by the peak streamwise vorticity. As shown earlier on Figure 4.6, the passage vortex for the contoured endwall case is stronger towards the endwall across the blade passage. Thus, the induced secondary velocities and the corresponding secondary kinetic energy are expected to be higher for the contoured endwall. This can be observed on Figure 4.6 and Figure 4.7. The application of endwall contouring creates a stronger pitchwise pressure gradient, which results in higher secondary kinetic energy near the endwall. This stronger gradient directs the high momentum fluid into the suction surface/endwall corner, in order to mitigate and therefore, reduce the losses (Harvey, 2008). The stronger secondary kinetic energy will generate additional losses as it dissipates moving downstream and will possibly also generate higher viscous losses inside the endwall boundary within the passage due to higher wall shear. However, it is expected that delaying and reducing the corner stall will result in lower total losses than if corner stall occurred earlier as for the flat endwall case. This is confirmed in Table 4.2 where the total loss coefficient for the contoured endwall is approximately 0.85 times the total loss for the flat endwall. It is worth noting that this application of endwall contouring might only be valid for highly-loaded compressor rows like CEGV-1, where the corner stall is a dominant source of losses. The stronger cross-flow works to mitigate the corner stall, and thus to reduce the losses. For more lightly loaded compressor rows, the corner stall might not be a source of losses as dominant and the additional losses due to the dissipation of the higher secondary kinetic energy resulting from the stronger cross-flow might not provide the same net benefit. It is conceivable that the optimum contouring for lightly loaded compressor rows might be one that reduces the cross-flow, such as in turbine rows.

The second region of high secondary kinetic energy corresponds to the flow driven up the suction surface of the blade by the secondary vortex and the subsequent interaction 
between the passage and counter vortices. The peak value for the flat endwall is approximately $60 \%$ higher than for the contoured endwall, which can be explained by the strength of the vortical structures. As observed on Figure 4.6, the passage and counter vortices of the flat endwall case are stronger in the area where they interact with each other compared to the contoured endwall case. Therefore, their interaction results in higher secondary velocities and higher secondary kinetic energy at this location. This can be observed on Figure 4.6 (a) and Figure 4.7 (a) at approximately $\mathrm{y} / \mathrm{s}=0.25$ and $\mathrm{z} / \mathrm{h}=0.12$ for the flat endwall. The peak of secondary kinetic energy is located near the peak region of total pressure deficit. The passage and counter vortices interact with each other to convect this high loss fluid from the endwall towards low loss regions, thereby increasing the mixing. The increase in mixing will result in generating higher losses as the flow moves downstream. For the contoured endwall, the core of secondary kinetic energy, also located in the area where the passage and counter vortices interact, is much smaller compared to the flat endwall case. This lower energy fluid will result in less loss being generated through mixing as the secondary kinetic energy dissipates moving downstream of the cascade.

Although the core of secondary kinetic energy near the endwall is stronger for the contoured endwall and results in loss generated through mixing, the application of endwall contouring is beneficial and results in lower overall total loss generated across the passage compared to the flat endwall test case as observed in Table 4.2. The smaller core of secondary kinetic energy being funnelled in low loss regions by the weaker vortices, along with the mitigation of the corner stall by a stronger cross-passage pressure gradient result in a reduction of total loss across the blade row compared to the flat endwall.

\subsubsection{Integrated Downstream Results}

The contour floods presented in the previous section provide a qualitative understanding of the flow physics downstream of the blade row. A more quantitative comprehension can be obtained by looking at the pitchwise and overall mass- or areaaveraged parameters. The pitchwise mass-averaged total pressure loss coefficient and secondary kinetic energy and the pitchwise area-averaged outlet flow angle measured at the downstream plane are shown on Figure 4.8 from the endwall to midspan. The overall mass- 
and area-averaged and the mixed-out mass-averaged exit flow results are summarized in Table 4.2 along with the summary of the inlet experimental results as presented in Table 4.1 and repeated for reference purposes.

a) Mass-averaged total pressure loss coefficient

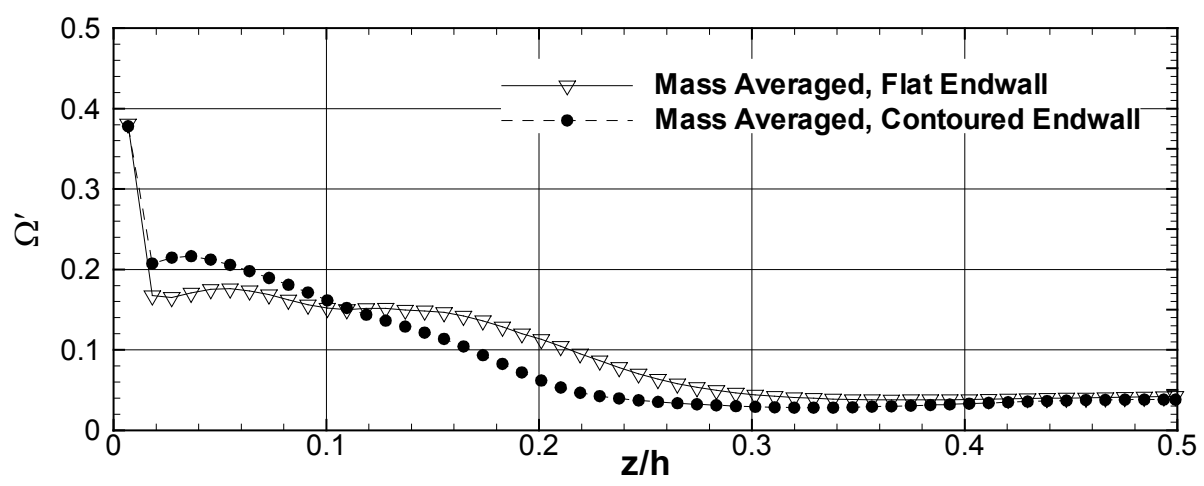

b) Area-averaged outlet flow angle

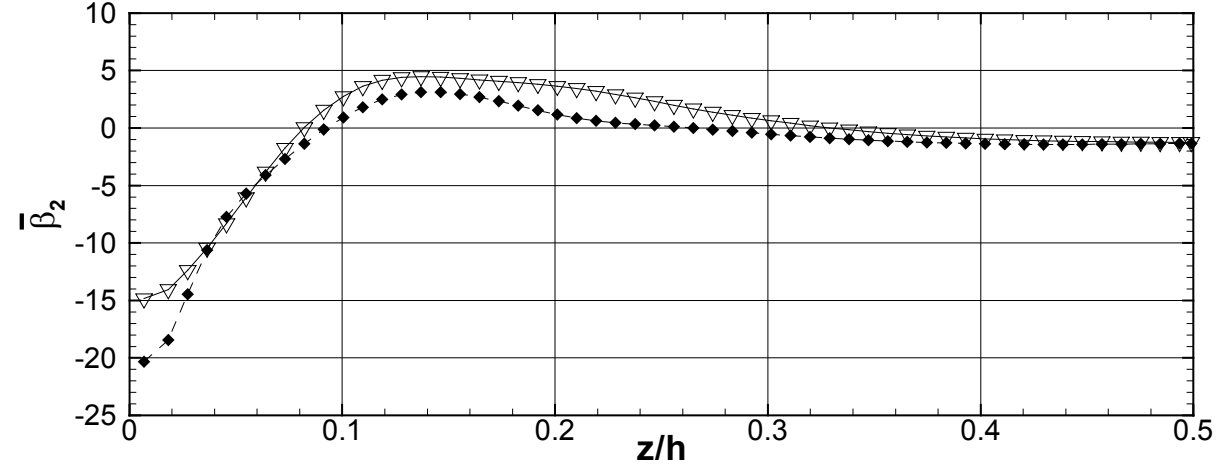

c) Mass-averaged secondary kinetic energy coefficient

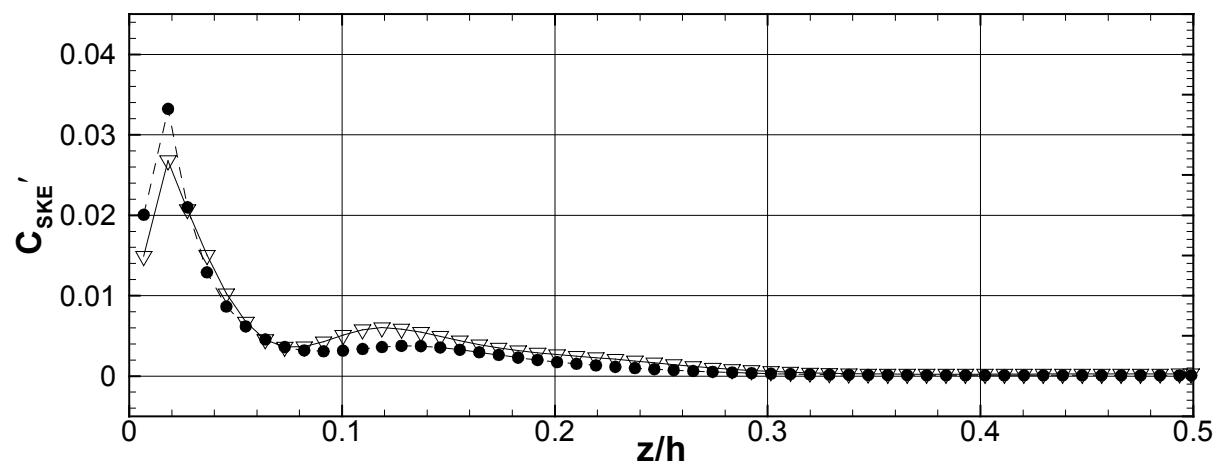

Figure 4.8: Measured pitchwise mass- and area-averaged results at $160 \% B_{x}$ plane: massaveraged total pressure loss coefficient (a), area-averaged outlet flow angle (b) and massaveraged secondary kinetic energy coefficient (c) at design incidence 
Table 4.2: Summary of experimental results at design incidence

ENDWALL

\begin{tabular}{|c|c|c|c|}
\hline LOCATION & $\begin{array}{c}\text { FLOW } \\
\text { QUANTITY }\end{array}$ & FLAT & CONTOURED \\
\hline \multirow[t]{7}{*}{ INLET } & $\delta / \mathrm{h}$ & 0.118 & 0.118 \\
\hline & $\delta^{*}[\mathrm{~mm}]$ & 2.57 & 2.25 \\
\hline & $\theta[\mathrm{mm}]$ & 1.99 & 1.80 \\
\hline & $\mathrm{H}$ & 1.29 & 1.25 \\
\hline & $\mathrm{C}_{\mathrm{q}, 1}{ }^{\prime \prime}$ & 0.982 & 0.987 \\
\hline & $\mathrm{C}_{\mathrm{q}, 1 \text { midspan }}{ }^{\prime}$ & 0.995 & 0.998 \\
\hline & $\mathrm{C}_{\mathrm{P} 0,1}{ }^{\prime \prime}$ & -0.011 & -0.010 \\
\hline OUTLET & $\mathrm{C}_{\mathrm{P} 0,2 \text { total }}{ }^{\prime \prime}$ & -0.089 & -0.077 \\
\hline \multirow[t]{11}{*}{$\mathrm{B}_{\mathrm{x}}=1.60$} & $\mathrm{C}_{\mathrm{P} 0,2 \text { profile }}{ }^{\prime}$ & -0.042 & -0.038 \\
\hline & $\mathrm{C}_{\mathrm{P} 0,2 \text { secondary }} "$ & -0.047 & -0.039 \\
\hline & $\Omega_{\text {total }}$ & 0.080 & 0.068 \\
\hline & $\Omega_{\text {profile }}$ & 0.041 & 0.038 \\
\hline & $\Omega_{\text {secondary }}$ & 0.039 & 0.030 \\
\hline & $\overline{\overline{\beta_{2}}}$ & -0.2 & -1.2 \\
\hline & $\overline{\bar{\beta}}_{2, \text { midspan }}$ & -1.3 & -1.3 \\
\hline & $\mathrm{C}_{\mathrm{q}, 2}{ }^{\prime \prime}$ & 0.506 & 0.492 \\
\hline & $\mathrm{C}_{\mathrm{q}, 2 \text { midspan }}{ }^{\prime}$ & 0.553 & 0.528 \\
\hline & $\mathrm{C}_{\mathrm{SKE}}{ }^{\prime \prime}$ & 0.003 & 0.002 \\
\hline & AVR & 1.106 & 1.070 \\
\hline \multirow[t]{10}{*}{ MIXED-OUT } & $\mathrm{C}_{\mathrm{P} 0, \text { total }}{ }^{\prime \prime}$ & -0.108 & -0.095 \\
\hline & $\mathrm{C}_{\mathrm{P} 0 \text {,profile }}$ & -0.045 & -0.041 \\
\hline & $\mathrm{C}_{\mathrm{P} 0 \text {,secondary }}{ }^{\prime \prime}$ & -0.062 & -0.054 \\
\hline & $\Omega_{\text {total }}$ & 0.099 & 0.086 \\
\hline & $\Omega_{\text {profile }}$ & 0.045 & 0.041 \\
\hline & $\Omega_{\text {secondary }}$ & 0.054 & 0.045 \\
\hline & $=\overline{\overline{\beta_{2}}}$ & -0.1 & -1.0 \\
\hline & $\overline{\bar{\beta}}_{2, \text { midspan }}$ & -1.2 & -1.3 \\
\hline & $\mathrm{C}_{\mathrm{q}}^{\prime \prime}$ & 0.453 & 0.436 \\
\hline & $\mathrm{C}_{\mathrm{q}, \text { midspan }}{ }^{\prime}$ & 0.542 & 0.519 \\
\hline
\end{tabular}


As observed for the total pressure loss coefficient (Figure 4.8 (a)), the contouring produces higher losses from the wall out to approximately $11 \%$ span, at which point the flat endwall produces more losses over the next $30 \%$ of span. From $11 \%$ to $40 \%$ span, a significant loss reduction is achieved by the contoured endwall. From $40 \%$ up to midspan, both endwalls produces approximately the same amount of loss, which confirms that the application of endwall contouring does not affect the midspan flow. The slight increase in loss observed in the last $10 \%$ of span shown for both configurations is a result of the inlet total pressure non-uniformity discussed in Section 4.2.1. From the overall mass-averaged results shown in Table 4.2, the values of the measured mass-averaged total, $\Omega_{\text {total }}$, and dynamic, $\Omega_{\text {secondary }}$, pressure loss coefficients for the endwall contouring are 0.85 and 0.76 times as large as the flat endwall values, respectively.

The pitchwise area-averaged outlet flow angle distribution in Figure 4.8 (b) shows that the blade row produces a typical over/underturning of the flow, similar to what was observed by other investigators such as Knezevici (2011), Harvey (2008), Hoeger et al. (2002), Reising and Schiffer (2009a) and Sonoda et al. (2008). The application of contouring increases the overturning near the endwall by a maximum of $5^{\circ}$ and reduces the maximum underturning by approximately $2^{\circ}$ compared with the flat endwall. These results are consistent with the inferences made from the contour floods in the previous sections. As shown on Figure 4.6, near the endwall, the secondary flow velocity vectors are stronger for the contoured endwall due to the increase strength of the cross-passage pressure gradient. This increased pressure gradient results in increasing the overturning of the flow compared to the flat endwall. However, from approximately $\mathrm{z} / \mathrm{h}=0.06$, the velocity vectors are stronger for the flat endwall as a result of the stronger passage and counter vortices. The fluid funnelled between these vortices is slightly underturned as it is moved towards the low-loss region. The weaker interaction between the passage and counter vortices, resulting from the application of contouring, decrease the underturning compared to the flat endwall. From $\mathrm{z} / \mathrm{h}$ $=0.4$ to 0.5 , the flow appears to be unaffected by the application of contouring. Both test cases resulted in a small overturning of about $1^{\circ}$ compared to the design outlet flow angle. As shown in Table 4.2, the overall area-averaged outlet flow angle of the contoured endwall case is more overturned by about $1^{\circ}$ compared to the flat endwall case. 
Figure 4.8 (c) shows the pitchwise mass-averaged secondary kinetic energy. The higher secondary kinetic energy near the wall for the contouring is a result of the flow overturning shown on Figure 4.8 (b) and will eventually cause additional pressure losses. However, as seen on Figure 4.8 (a), the increased strength of the cross-flow is beneficial by driving the high momentum fluid near the endwall and up the blade surface, thus delaying the corner stall and its associated losses. The second peak observed around $\mathrm{z} / \mathrm{h}=0.12$ is a result of the flow underturning and of the spanwise flow funnelled into the low-loss region. As observed, the peak is higher for the flat endwall, which was expected due to the stronger interaction between the passage and counter vortices in this case. Therefore, the application of endwall contouring has the effect of increasing the secondary kinetic energy near the endwall (due to overturning) and reducing it from approximately $\mathrm{z} / \mathrm{h}=0.08$ to $\mathrm{z} / \mathrm{h}=0.3$. Overall, the measured mass-averaged $C_{S K E}$ of the contoured endwall has been reduced by approximately one third of the flat endwall value.

The overall mixed-out mass-averaged exit flow results are shown in Table 4.2. As discussed in Chapter 3, the mixed-out losses are calculated from a control volume that extends at constant area from the downstream measurement plane to a hypothetical mixedout plane where uniform conditions exist. On this hypothetical plane, the secondary kinetic energy is completely dissipated and appears as total pressure losses. In addition to the dissipation of the secondary kinetic energy, mixing losses are generated through the recovery of static pressure and the mixing-out of the non-uniformities in the primary kinetic energy. The mixed-out mass-averaged total, $\Omega_{\text {total }}$, and dynamic, $\Omega_{\text {secondary }}$, pressure loss coefficients for the endwall contouring case are 0.87 and 0.83 times as large as the flat endwall values, respectively. The mixed-out area-averaged outlet flow angles for both endwalls are slightly smaller than at the measurement plane. The overall area-averaged outlet flow angle of the contoured endwall is more overturned by $0.9^{\circ}$ compared to the flat endwall one. 


\subsection{Summary of Results and Conclusions}

Experimental results have been presented documenting changes to the flow field of a compressor exit guide vane as a result of the application of non-axisymmetric endwall contouring. Following the investigations presented in this chapter, the results confirmed that endwall contouring could be used to reduce endwall losses.

Endwall contouring increases the intensity of the cross-passage flow near the endwall in order to drive high momentum fluid towards the suction surface/endwall corner and up the blade surface in order to reduce or delay the formation of the corner stall. Corner stall can be a significant source of losses in a highly-loaded compressor blade row. Although increasing the strength of the cross-passage flow near the endwall increases the strength of the passage vortex in this region and will tend to increase the secondary losses, it was concluded that delaying the formation of the corner stall was more beneficial than offset these losses. As shown, the overall mass-averaged secondary losses across the passage were reduced following the application of endwall contouring.

The oil surface flow visualisations presented provided a detailed interpretation of the three-dimensional flow field near the walls of the blade passage and gave insights into how the flow field is affected by the application of endwall contouring.

The results presented in this chapter demonstrated that using endwall contouring can reduce endwall losses at the design incidence. The next chapter will examine the effect of varying inlet flow incidence on the flow field and the performance of endwall contouring in such conditions. 


\section{Chapter 5 Results and Discussion}

\section{Secondary Flows at Off-Design Incidence}

\subsection{Introduction}

This chapter presents the results of the investigations on the flow field of the CEGV-1 compressor cascade at an off-design incidence value of $+7^{\circ}$. The blade row studied is rather highly-loaded and the incidence will result in increasing the loading and the losses. Therefore, one of the objectives of this investigation was to have a better understanding on how the flow field is affected as incidence is greatly increased and how it affects the loss generation. Another objective was to investigate the effects of endwall contouring at offdesign incidence in order to see if the potential benefit observed at design incidence was also present at off-design. The testing was conducted at an inlet Reynolds number of 150,000 based on the axial chord and the inlet centerline velocity. The current chapter examines the secondary flows of the compressor cascade with and without the application of endwall contouring.

Section 5.2 provides the experimental results for both planar and contoured endwalls. The inlet flow, the blade loading distributions and the pressure probe results are provided in the separate sub-sections. Finally, the last section summarizes the findings and conclusions. 


\subsection{Experimental Results}

\subsubsection{Inlet Conditions}

The boundary layer was traversed from the endwall to midspan using a Pitot probe located 1.2 axial chords upstream of the leading edge of the cascade at mid-passage. The inlet endwall boundary layer parameters and mass-averaged pressure coefficients at $7^{\circ}$ of incidence are given in Table 5.1. The design incidence parameters are repeated from Table 4.1 for comparison purposes. The non-uniformities present in the inlet flow at design incidence are still present at off-design incidence. The non-uniformities were documented by traversing the inlet plane using a three-hole pressure probe over one blade pitch and over half the full blade span. The displacement thickness $\left(\delta^{*}\right)$ and the momentum thickness $(\theta)$ were obtained by integrating the inlet boundary layer from the endwall to the point of maximum velocity. The boundary layer shape factors $(\mathrm{H})$ for both endwall configurations are typical of turbulent boundary layers with zero pressure gradient.

Table 5.1: Summary of experimental results at $7^{\circ}$ of incidence measured $1.2 B_{x}$ upstream of the blade leading edge.

\begin{tabular}{|c|c|c|c|c|c|}
\hline \multirow[b]{2}{*}{ LOCATION } & \multirow[b]{2}{*}{$\begin{array}{c}\text { FLOW } \\
\text { QUANTITY }\end{array}$} & \multicolumn{2}{|c|}{ DESIGN INCIDENCE } & \multicolumn{2}{|c|}{$7^{\circ}$ INCIDENCE } \\
\hline & & FLAT & CONTOURED & FLAT & CONTOURED \\
\hline INLET & $\delta / \mathrm{h}$ & 0.118 & 0.118 & 0.132 & 0.138 \\
\hline & $\delta^{*}[\mathrm{~mm}]$ & 2.57 & 2.25 & 2.83 & 2.70 \\
\hline & $\theta[\mathrm{mm}]$ & 1.99 & 1.80 & 2.22 & 2.15 \\
\hline & $\mathrm{H}$ & 1.29 & 1.25 & 1.27 & 1.26 \\
\hline & $\mathrm{C}_{\mathrm{q}, 1}{ }^{\prime \prime}$ & 0.982 & 0.987 & 0.985 & 0.973 \\
\hline & $\mathrm{C}_{\mathrm{q}, 1 \text { midspan }}{ }^{\prime}$ & 0.995 & 0.998 & 1.003 & 0.992 \\
\hline & $\mathrm{C}_{\mathrm{P} 0,1}{ }^{\prime \prime}$ & -0.011 & -0.010 & -0.021 & -0.022 \\
\hline
\end{tabular}

As seen, the thicknesses of the boundary layers are slightly larger at off-design incidence. As discussed in the previous chapter, the differences observed in the boundary layer parameters for a given incidence between both endwalls are mainly an indication of 
scatter. However, different potential effects from the blade and endwall configurations, different flow control surfaces and blockage configurations, and small differences in the endwall boundary layer bleed flows upstream of the cascade could also be sources of differences observed between the two endwall configurations. The differences in massaveraged dynamic and total pressure coefficients observed between both endwalls at $7^{\circ}$ incidence are within probe measurement error.

\subsubsection{Blade Loading Distributions}

As discussed in the previous chapter, the size and strength of the secondary flows are influenced by the blade loading, its distribution and the presence of separation bubbles on the blade surfaces. Figure 5.1 shows the loadings for both flat and contoured endwalls test cases at the two values of incidence. The loadings are shown at midspan (Figure 5.1 (a)), 20\% span (Figure 5.1 (b)) and 5\% span (Figure 5.1 (c)). The loadings at design incidence have been repeated for comparison purposes.

As seen on Figure 5.1 (a), increasing incidence has significant effect on the static pressure distributions, especially on the suction surface of the blade. The suction peak is located at $x / B_{x}=0.05$, which is slightly closer to the leading edge of the blade than at design incidence. As observed, the difference between the suction and pressure surfaces static pressure coefficients is larger at $7^{\circ}$ of incidence than at design incidence up to approximately $x / B_{x}=0.12$. Therefore, increasing the incidence resulted in increasing the loading in the front of the blade. From the loadings at $7^{\circ}$ incidence, a region of approximately constant pressure is observed at midspan on the suction surface of the blade. This constant pressure plateau suggests boundary layer separation. However, unlike at design incidence, this apparent separation is not a separation bubble since there is no sign of reattachment. The constant pressure plateau is observed from approximately $x / B_{x}=0.5$ up to the trailing edge of the blade and corresponds to a breakdown in diffusion and presumably, flow separation. Unfortunately, flow visualisations at off-design incidence have not been conducted in order to verify that the constant static pressure plateau is in fact a region of two-dimensional separation. As it will be discussed later in Section 5.2.3.1, the larger two-dimensional wake at midspan also suggests that two-dimensional separation is present on the suction surface of 
the blade. This would result in an increase in the profile pressure losses, as it will be shown later in Section 5.2.3.4.
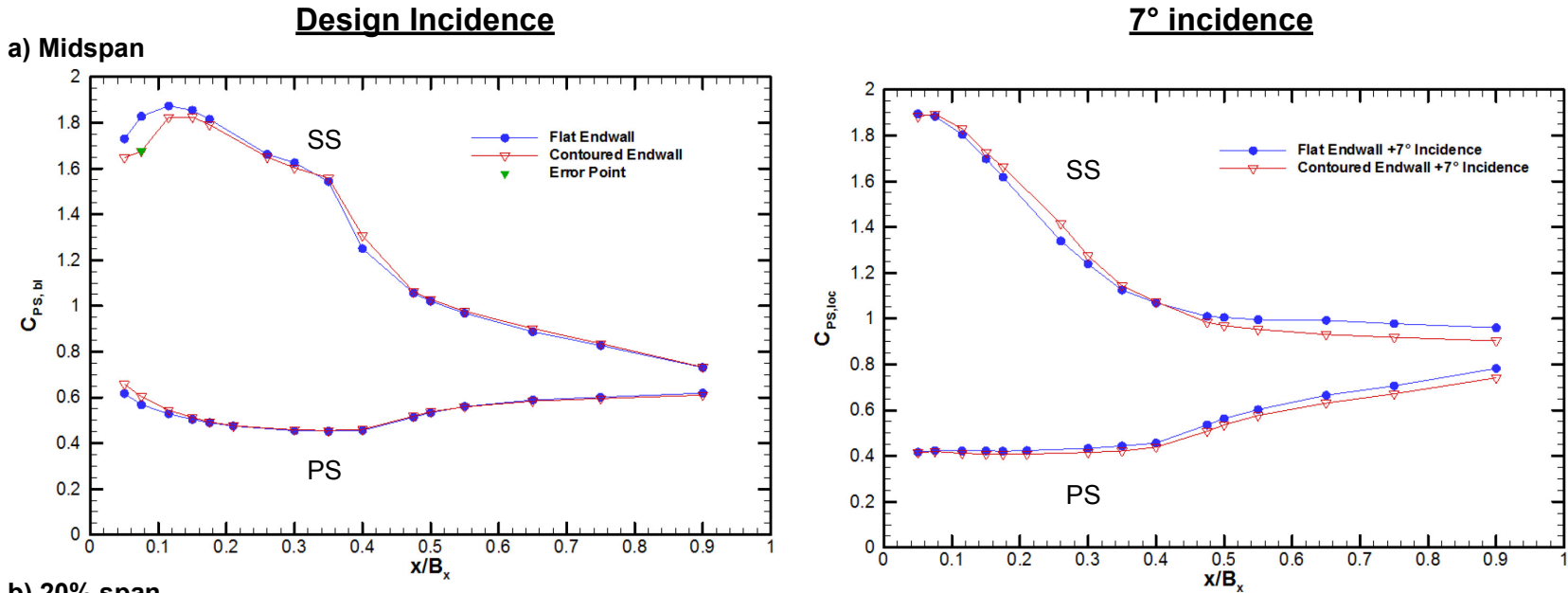

b) $20 \%$ span
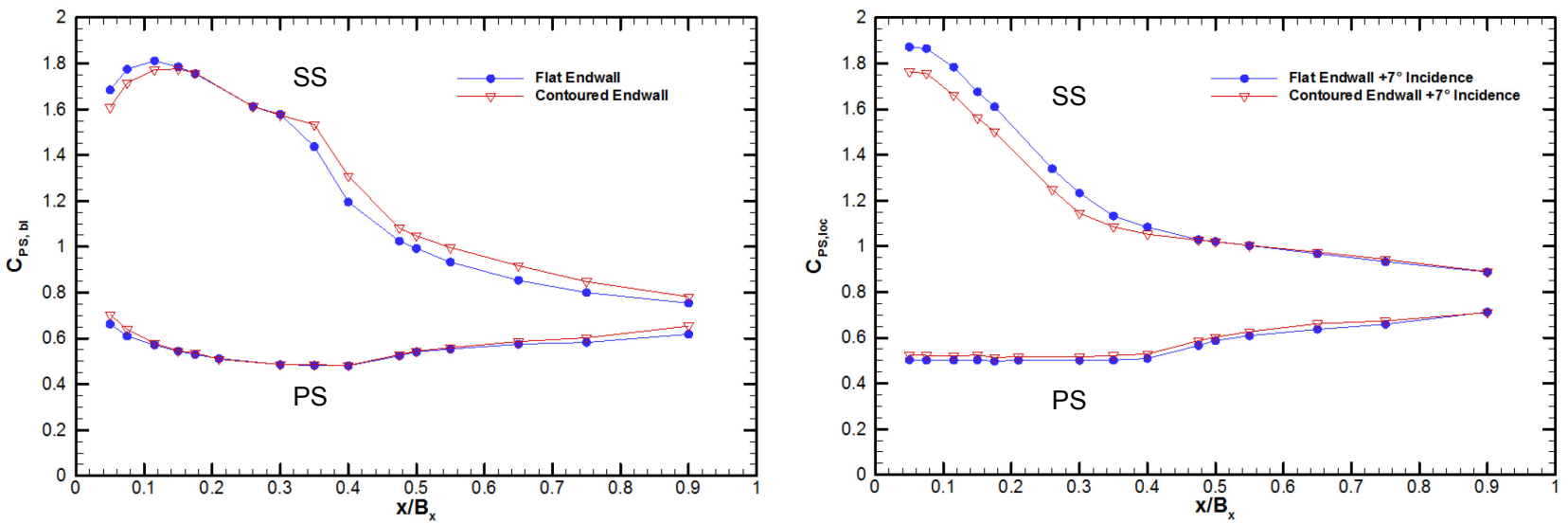

c) $\mathbf{5 \%}$ span
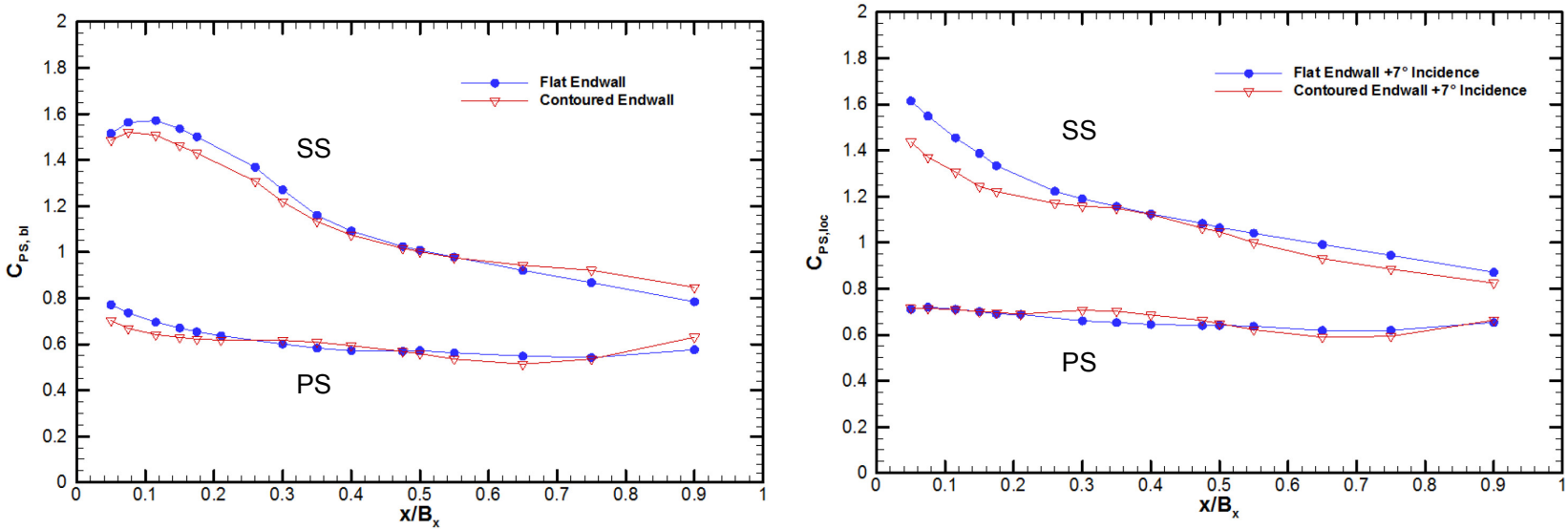

Figure 5.1: Blade surface static pressure distribution at $\mathrm{z} / \mathrm{h}=0.50(\mathrm{a}), \mathrm{z} / \mathrm{h}=\mathbf{0 . 2 0}$ (b) and $\mathrm{z} / \mathrm{h}=\mathbf{0 . 0 5}$ (c) at design and $7^{\circ}$ incidences 
Similar observations can be made for the blade loadings at $20 \%$ and $5 \%$ span shown on Figure 5.1 (b, right) and Figure 5.1 (c, right), respectively. The suction peaks for both endwalls at $20 \%$ span are slightly closer to the leading edge of the blade compared to the suction peaks at design incidence. For the contoured endwall, the suction peak value is approximately the same as at design incidence, but the blade is slightly more front loaded. However, for the flat endwall, the blade is also slightly more front loaded, but the suction peak is higher than at design incidence. This means that the blade is more highly-loaded at the higher incidence with the flat endwall. At 5\% span, the measured data do not show a clear definition of the suction peak, but it appears to have moved closer to the leading edge of the blade, which would make the blade more front-loaded near the endwall. As at $20 \%$ span, the blade is more highly-loaded with the flat endwall than with the contoured endwall in the first $30 \%$ axial chord. The differences observed between the loadings at $7^{\circ}$ incidence suggest that the interaction of the suction surface boundary layer and the endwall cross-flow is altered by the application of contouring, therefore modifying the static pressures on the blade surface at these spanwise locations.

Unlike at midspan, after the suction peak, there is no plateau of static pressure on the suction surface of the blade that would suggest two-dimensional flow separations at 20 and 5\% spans. From Figure 5.1 (b, right) and Figure 5.1 (c, right), it appears that the flow is still diffusing moving downstream of the passage. This different behaviour is likely due to the secondary flows in the passage affecting the surface static pressures. At $20 \%$ span, even if the suction surface static pressure coefficient decreases moving towards the trailing edge, the rate of diffusion is small, suggesting a possible suction surface flow separation. This noted behaviour is however not observed at 5\% span, which suggests that it is prevented by the interaction of the suction surface boundary layer with the endwall cross-flow moving up the blade surface.

From Figure 5.1 (a, right), it can also be observed that the blades are slightly underturning the flow at $7^{\circ}$ incidence, which is noted by the higher outlet dynamic pressure than at design incidence. The blades are not decelerating the flow as much as for the design cases. This reduction in diffusion is consistent with the previously noted flow separation on the suction surface of the blade. The outlet dynamic pressures for both $20 \%$ and $5 \%$ spanwise 
positions (Figure 5.1 (b, right) and Figure 5.1 (c, right)) are also slightly higher than at design incidence. Therefore, this observation suggests that, at $7^{\circ}$ incidence, the blades are not diffusing the flow as effectively as at design incidence.

It can be observed on Figure 5.1 (a, right) that there is a slight difference in the blade loadings between the flat and contoured endwalls from approximately half the blade chord to the trailing edge. This difference might not be due to the endwall contouring, but instead, to the stronger secondary flows of the opposite, uncontoured endwall. As it will be shown in Section 5.2.3.1, the secondary flows in the opposite half-span of the measured passage are stronger, pushing the two-dimensional region closer to the measured endwall. This could possibly explain the difference in loading noted towards the trailing edge of the blade. Unlike at midspan, the loadings in the aft part of the blade at 20 and 5\% spans are approximately the same between both endwalls, suggesting that the effect of the unequal secondary flows of the opposite endwalls may be reduced. The slightly higher loading at $5 \%$ span with the flat endwall might simply be due to the effects of endwall contouring in this area.

\subsubsection{Downstream Flow Field Measurements}

\subsubsection{Total Pressure Distributions}

Figure 5.2 shows colour flood contour plots of total pressure deficit coefficient for both flat and contoured endwall test cases as measured on a plane located 1.6 axial chords downstream of the blade leading edge. The contour plots at design incidence have been repeated from Figure 4.5 for comparison purposes.

The results are plotted over 1.2 blade pitches and from the endwall to half span with the appropriate pitch-to-chord ratios (see Table 3.2). The projections of the trailing edges are shown as dashed lines at $\mathrm{y} / \mathrm{s}=0$ and $\mathrm{y} / \mathrm{s}=1$. Here, the high loss regions are represented by red, the low loss regions by blue and the no loss regions by white.

Both floods at $7^{\circ}$ of incidence (Figure 5.2 (b)) show similar general features such as one region of elevated loss near the endwall closer to the suction surface. The magnitude of the peak loss is also similar, with a value hovering around $C_{P 0}=-0.45$. However, some differences can be observed between the secondary flows of the two endwalls. 
Flat Endwall

a) Design Incidence

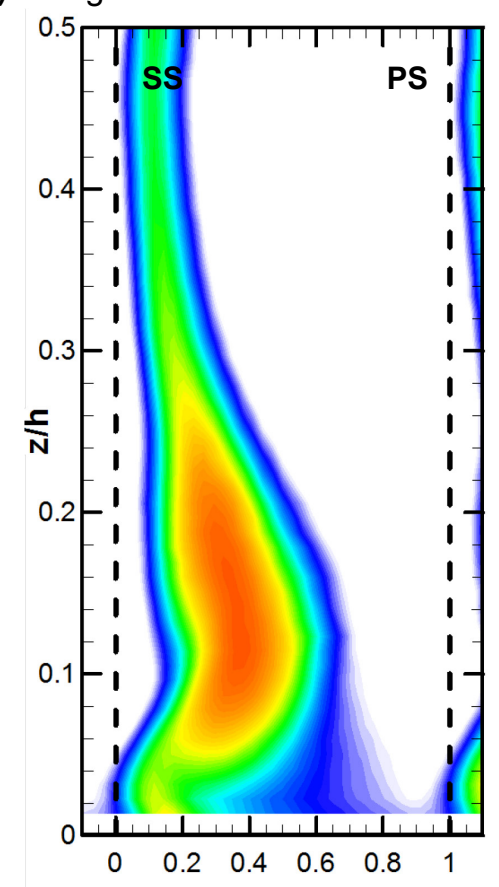

b) $7^{\circ}$ incidence $\quad \mathrm{y} / \mathrm{s}$

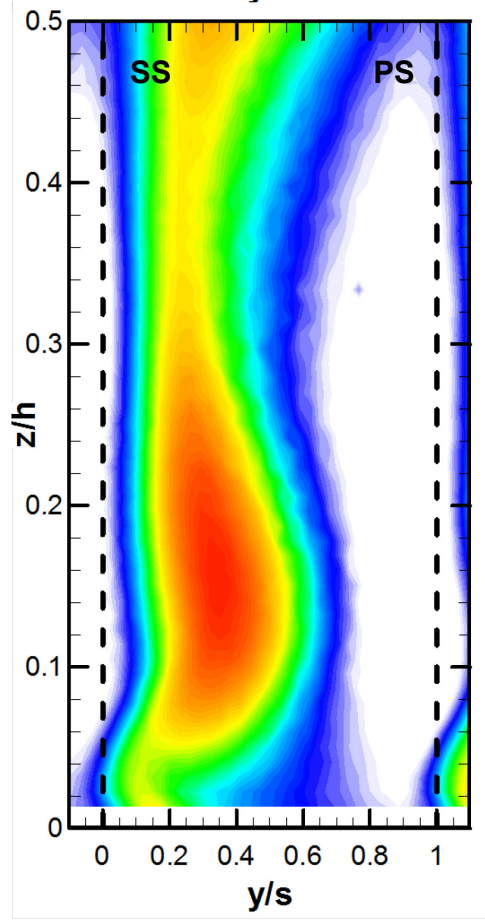

\section{Contoured Endwall}
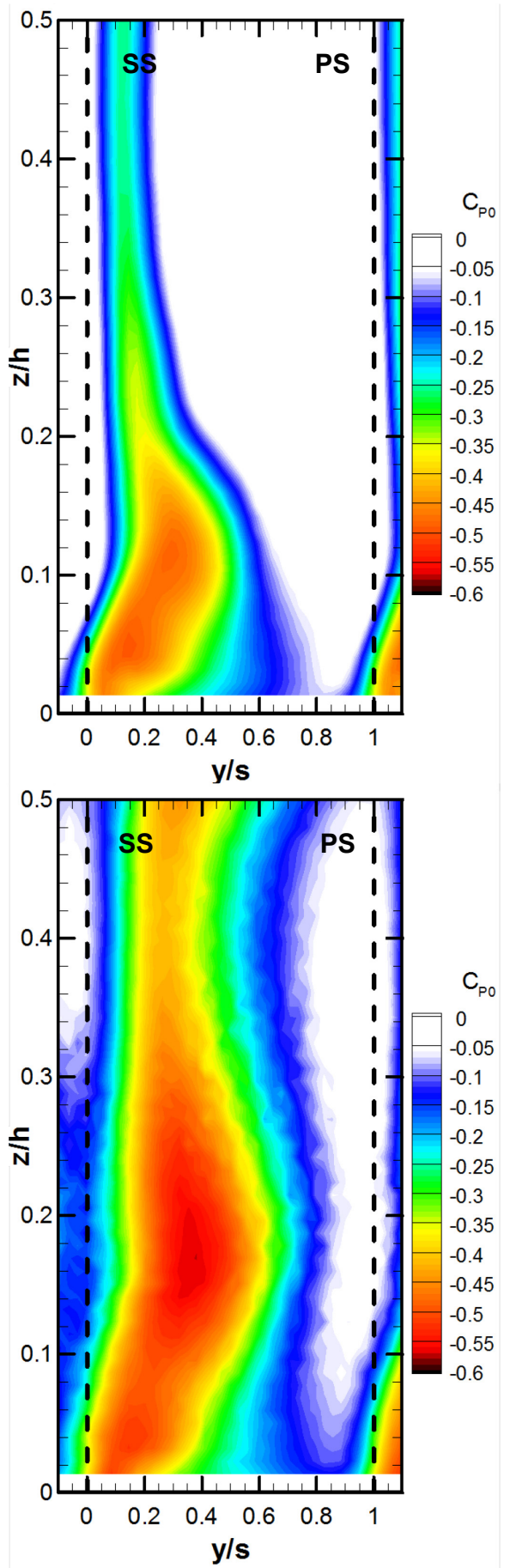

Figure 5.2: Total pressure deficit coefficient $\left(C_{P O}\right)$ floods at $1.60 B_{x}$ for the flat (left) and contoured (right) endwall configurations at design (a) and $7^{\circ}$ (b) incidences 
For the flat endwall, the elevated region of loss extends from approximately $y / s=0.20$ to 0.60 and $\mathrm{z} / \mathrm{h}=0.06$ to 0.30 , with a peak loss located at approximately $\mathrm{y} / \mathrm{s}=0.30$ and $\mathrm{z} / \mathrm{h}=$ 0.15 . For the contoured endwall, the elevated loss region extends from $\mathrm{y} / \mathrm{s}=0.00$ to 0.60 and $\mathrm{z} / \mathrm{h}=0.015$ to 0.36 with a slightly more elevated peak loss located at approximately $\mathrm{y} / \mathrm{s}=$ 0.35 and $\mathrm{z} / \mathrm{h}=0.16$.

For the flat endwall (Figure $5.2(\mathrm{~b}, \mathrm{left})$ ), although the magnitude of the peak loss is similar to the one at design incidence, the core of the peak loss is larger, which will result in larger integrated loss in the passage. Also, the low loss region (blue) is significantly increased and the no loss region (white) is significantly decreased compared to the total pressure loss deficit flood at design incidence shown on Figure 5.2 (a, left). Similar observations can be made for the contoured endwall plot. The peak magnitude of the elevated loss region is slightly increased compared to the design incidence one; however, the area occupied by the high-loss fluid is significantly increased. The increase in magnitude and area of the elevated loss region results in a large increase in downstream integrated pressure losses, which will be shown in Section 5.2.3.4.

The area and magnitude of elevated loss for the flat endwall at $7^{\circ}$ of inlet flow incidence are slightly increased compared to the design incidence ones. However, the shape of the total pressure deficit coefficient flood is similar. For the contoured endwall, as observed on Figure 5.2 (b, right), the elevated loss region resulting from an inlet flow at $7^{\circ}$ is significantly altered from the one at design incidence, suggesting an increase in the integrated downstream losses as will be confirmed later in Section 5.2.3.4. This suggests that the application of endwall contouring might not be as valuable at off-design incidence. This is presumably due to the fact that the endwall curvatures have been optimized for design incidences. Therefore, at large off-design incidences, the contouring alters the secondary flow fields in such manner that to increase the total pressure deficit, as confirmed by the integrated values presented in Table 5.2.

Unlike the contour plots of total pressure deficit coefficient at design incidence shown on Figure 5.2 (a), the contour plots at $7^{\circ}$ of incidence do not have a two-dimensional wake extending up to midspan. For the off-design cases, it appears that the flow is far from symmetric about midspan and that the secondary flows from the opposite half-span of the 
passage are stronger. One of the consequences is that the stronger secondary flows create a larger blockage in the non-measured half of the passage, thus reducing its local mass flow and increasing it in the measured half for a given total inlet mass flow. This larger blockage also forces the flow to converge at a lower spanwise value of $\mathrm{z} / \mathrm{h}$ than midspan and to push the two-dimensional flow closer to the measured endwall. From Figure 5.2 (b), the twodimensional regions for the flat and contoured endwalls are extending from approximately $\mathrm{z} / \mathrm{h}=0.34$ to 0.42 and $\mathrm{z} / \mathrm{h}=0.37$ to 0.45 , respectively. It can also be noted that the twodimensional wakes of both endwalls are significantly larger than at design incidence (shown on Figure 5.2 (a)). These wider wakes represent additional losses and will have a significant impact on the profile losses at off-design incidence, as it will be shown on Table 5.2. A probable explanation for these larger wakes is the possible two-dimensional flow separation on the suction surface of the blade observed at midspan on Figure 5.1 (a, right). This suction surface flow separation, along with the trailing edge separation, could explain the increase in losses generated downstream of the trailing edge of the blade away from the endwall region.

Investigations of the asymmetric flow around midspan were performed by traversing the inlet plane of the non-measured half of the passage with the three-hole pressure probe to verify the flow uniformity. The measurements showed that the inlet flow incidence was approximately 3 to 4 degrees higher than for the measured half of the passage. As noted previously, a higher inlet flow incidence results in stronger secondary flows and in higher secondary losses. Therefore, the increased blockage in the non-measured half of the passage forces the flow to converge at a lower spanwise value of $\mathrm{z} / \mathrm{h}$ than midspan. However, the investigations performed were inconclusive to determine the source of the higher inlet flow incidence in the non-measured half of the passage. For the remaining analyses, it is assumed that this asymmetry does not affect the structure of the secondary flows but only the twodimensional region, thus allowing data analysis of the vorticity and secondary kinetic energy fields to be performed. However, to integrate the downstream results presented in Section 5.2.3.4, it was required that the flow fields near midspan be two-dimensional. Thus, the twodimensional wakes were extended for integration purposes from $\mathrm{z} / \mathrm{h}=0.35$ and $\mathrm{z} / \mathrm{h}=0.40$ to midspan for the flat and contoured endwalls, respectively. The modified wakes are shown on Figure 5.3. These modified outlet flow fields were only used for integration purposes. 

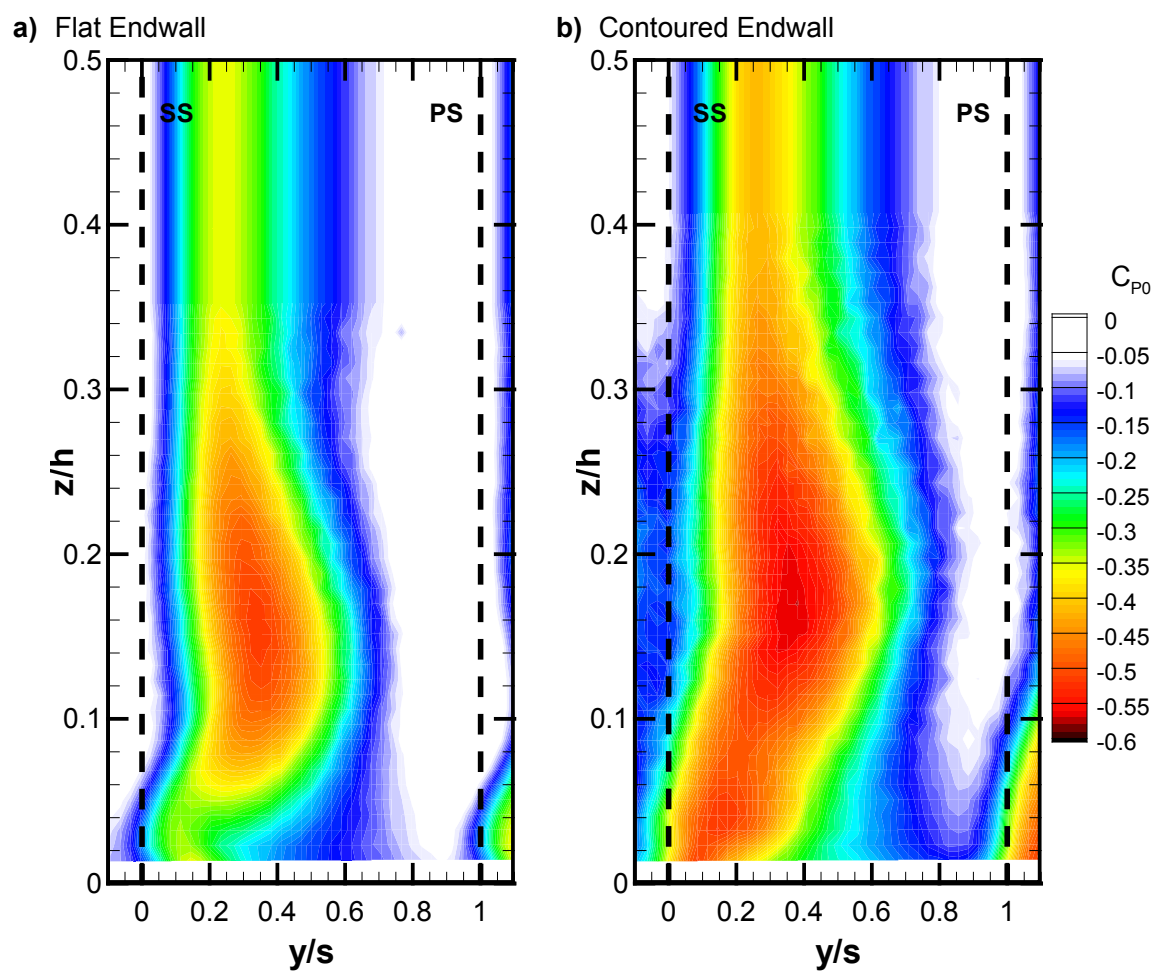

Figure 5.3: Total pressure deficit coefficient $\left(C_{\mathrm{PO}}\right)$ floods with straightened wakes for the flat (a) and contoured (b) endwall configurations at $7^{\circ}$ of incidence

\subsubsection{Streamwise Vorticity Fields}

Figure 5.4 shows the secondary velocity vectors surimposed on a colour flood of streamwise vorticity coefficient. The contour plots at design incidence have been repeated from Figure 4.6 for comparison purposes. The secondary velocity vectors represent the components of velocity on a plane normal to the primary flow direction. The streamwise vorticity is derived from the incompressible Euler equations as per Gregory-Smith et al. (1988). This approximation is used as it allows all components of the vorticity vector to be estimated from measurements on a single traverse plane. The validity of this approximation in viscous flows has been studied by Yaras and Sjolander (1990). It was concluded that the error in vorticity was very small everywhere except in the vortex core where the velocity gradient is changing most rapidly and where the viscous effects are most important. However, even in this region, it was found that the losses are small enough to use this approximation. 


\section{Flat Endwall}

a) Design Incidence

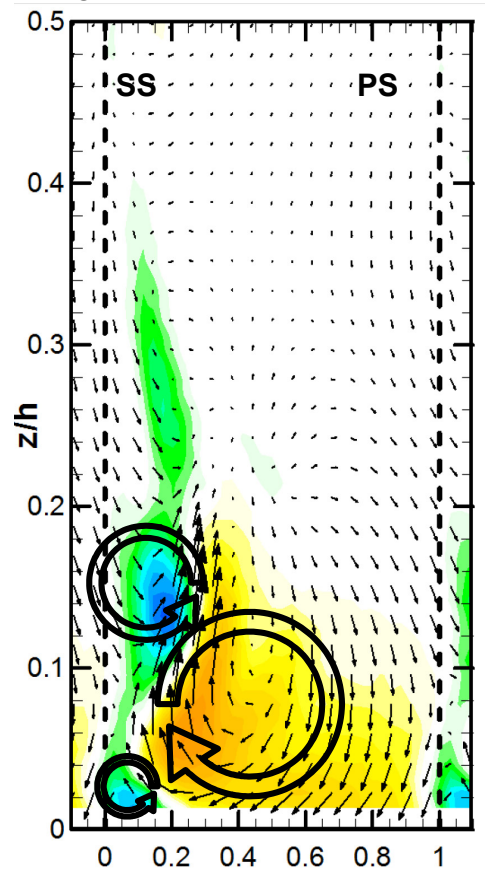

b) $7^{\circ}$ incidence

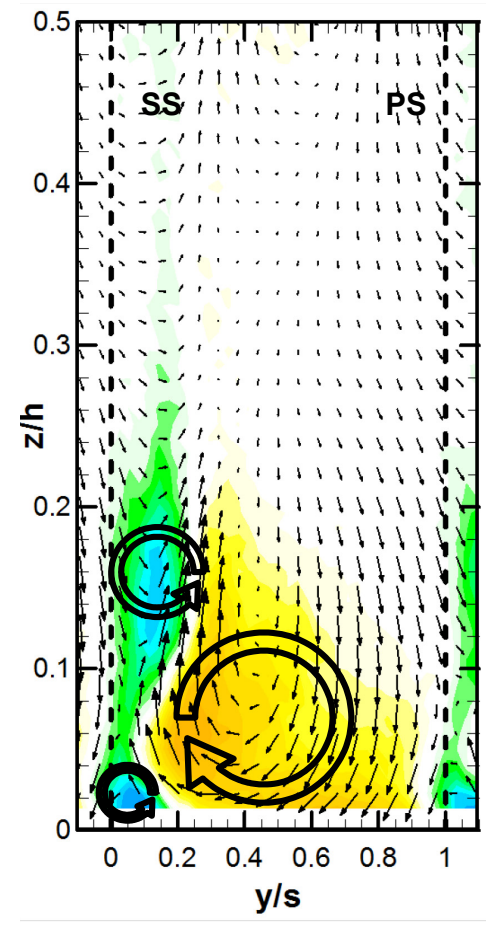

\section{Contoured Endwall}
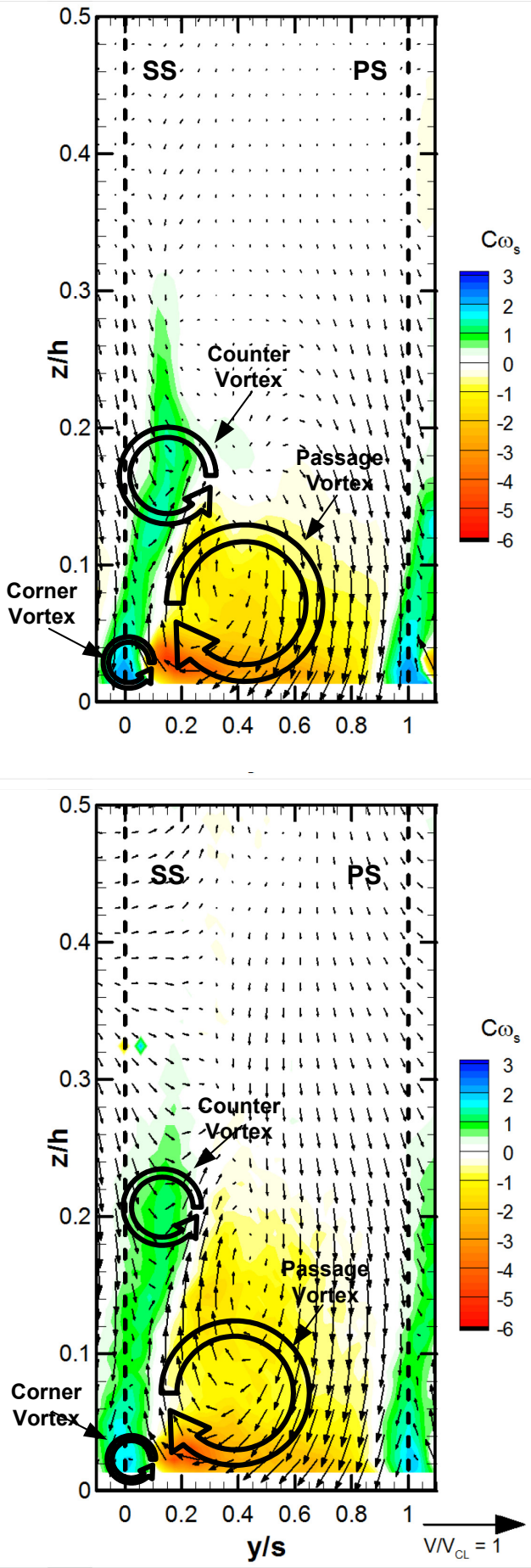

Figure 5.4: Streamwise vorticity coefficient $\left(\mathrm{C \omega}_{\mathrm{s}}\right)$ floods overlaid with secondary velocity vectors for the flat (left) and contoured (right) endwalls at design (a) and $7^{\circ}$ (b) incidences 
The streamwise vorticity represents the local rotation of the fluid relative to the streamwise direction. It is used to identify the different vortical structures and to quantify their strength and sense of rotation (Knezevici, 2011). The strength is obtained here from the magnitude of the streamwise vorticity coefficient, defined in equation (3.24) in Section 3.9.4.2. The streamwise vorticity is defined using the right hand rule, such that yellow and red regions indicate a negative rotation about the streamwise axis, while the green and blue regions represent a positive rotation.

Three main vortical structures can be identified on Figure 5.4: the passage vortex, the counter vortex and the corner vortex. The passage vortex has negative vorticity about the streamwise direction. The counter vortex and the corner vortex both have positive vorticity. In general, the flow fields for both test cases appear similar, but several features are worth discussing.

The passage vortex originates from the inlet endwall boundary layer. It moves from the pressure surface of the blade towards the suction surface of the adjacent blade as shown on Figure 5.4. The negative vorticity shows that the passage vortex of the flat endwall slightly increases in size, mostly in the spanwise direction, and in its total magnitude as the incidence is increased, as seen by comparing Figure 5.4 (b, left) and Figure 5.4 (a, left). However, the peak negative vorticity at off-design incidence, located approximately at $\mathrm{y} / \mathrm{s}=0.25$ and $\mathrm{z} / \mathrm{h}=$ 0.07 , is slightly lower than at design incidence. This suggests that the strength of the passage vortex, here defined as the peak streamwise vorticity, is reduced as the inlet flow incidence is increased. For the contoured endwall, the negative vorticity shows that the passage vortex significantly increases in size and in its total magnitude as the incidence is increased, as it can be seen from Figure 5.4 (b, right) compared to Figure 5.4 (a, right). The peak negative streamwise vorticity is located approximately at $\mathrm{y} / \mathrm{s}=0.20$ and $\mathrm{z} / \mathrm{h}=0.03$ and is slightly lower than the peak at design incidence. This suggests that the passage vortex for the contoured endwall is, although being significantly larger in size and magnitude, weaker at off-design incidence compared to design incidence. Also, unlike at design incidence, the application of endwall contouring does not reduce the size and magnitude of the passage vortex present with the flat endwall. As seen, the passage vortex for the contoured endwall extends further along the span and pitch of the passage compared to the flat endwall. 
A possible explanation for the reduced strength of the passage vortex for both endwalls is that the duration of the interaction between the passage and counter vortices increases at higher incidences due to the reduction in streamwise velocities. Benner (2003) suggested that an increase in the duration of the interaction of the vortices increases their mixing. The increase in mixing results in a reduction of the vortex strength, which could explain the slight reduction in the peak magnitude of streamwise vorticity seen on Figure 5.4 (b).

It can also be seen that the secondary velocity vectors associated with the passage vortex are slightly stronger at off-design incidence for both endwalls, therefore resulting in higher secondary kinetic energy and stronger secondary flow. This suggests that larger total pressure losses will result from the dissipation of this higher secondary kinetic energy as the flow moves downstream. This will be confirmed in Section 5.2.3.4 from the downstream integrated results. As for the design incidence, the secondary velocity vectors for the contoured endwall are stronger near the pressure surface compared to the flat endwall case. This is a result of the contoured endwall trying to manipulate the cross-passage flows to force the loss regions closer to the endwall and to reduce the mixing in the inviscid core. However, unlike at design incidence for the contoured endwall, the local acceleration of the secondary velocity vectors does not succeed in modifying the cross-passage flow such as to push the loss core closer to the suction surface/endwall corner, as it can be seen by comparing Figure 5.2 (b, right) with Figure 5.2 (a, right). The likely explanation is that, since the endwall curvatures were optimized for the design inlet flow angle, at off-design values of incidence, the cross-passage flow is not manipulated as effectively, resulting in different endwall crossflow behaviour.

The second main vortex present is the counter vortex, which is made up of fluid from the suction side of the horseshoe vortex moving up the blade surface and likely from some blade surface boundary layer fluid involved in the separation on the suction surface. For the off-design incidence cases, flow separation on the blade suction surface can not be confirmed as flow visualisations were not conducted, but the sudden breakdown in diffusion and the suction surface static pressure plateau strongly suggest it. Unlike at design incidence, the application of endwall contouring does not significantly affect the size and magnitude of the counter vortex at $7^{\circ}$ of incidence compared to the flat endwall, but it does reduce its strength 
as shown on Figure 5.4 (b). Furthermore, it can be seen that the size and magnitude of the counter vortices for both endwalls are slightly increased at off-design incidence compared to design incidence. However, as for the passage vortices, the strength of the counter vortices is reduced. One possible explanation for the weaker vortices is the same as for the passage vortex, namely the increased duration of the interaction between the passage and counter vortices. The longer duration results in an increase in mixing between the two vortices, therefore reducing their strength. It is also worth noting that the location of the counter vortices for both endwalls at off-design incidence is closer to midspan due to the larger size and magnitude of the passage vortices.

The last vortical structure, namely the corner vortex, can be observed on Figure 5.4 (b) near the endwall and to the right of the trailing edge projection. The core of the corner vortex is located at approximately $\mathrm{y} / \mathrm{s}=0.1$ and $\mathrm{z} / \mathrm{h}=0.02$ for the flat endwall case and $\mathrm{y} / \mathrm{s}=0.0$ and $\mathrm{z} / \mathrm{h}=0.02$ for the contoured endwall case. The increased strength of the passage vortex near the endwall for the contoured endwall confines the corner vortex to a slightly smaller area than for the flat endwall. However, unlike at design incidence, the corner vortex at $7^{\circ}$ of incidence is weaker for the contoured endwall compared to the flat endwall. At design incidence, the endwall curvatures were optimized to manipulate the cross-passage flow such as to intensify the corner vortex. As discussed above, the cross-passage flow is not affected in the same manner at off-design incidence, thus possibly explaining the different interaction between the passage and the corner vortices and therefore, the lower strength. This observation appears to be valid only for the contoured endwall. The increase in inlet flow incidence does not seem to have much effect on the size, magnitude and strength of the corner vortex for the flat endwall.

The interaction between the passage vortex and the counter vortex for the flat endwall results in the accumulation of high loss fluid between the two vortices. The rotation of these two vortical structures results in high secondary velocity vectors, which tend to sweep the endwall fluid into the inviscid core, increasing mixing and losses with downstream distance. This region of high secondary velocity corresponds to the loss core observed on Figure 5.2 (b). As for the design incidence cases, the interaction between the two vortical structures is also weaker for the contoured endwall at the increased incidence. As shown on Figure 5.4 (b, 
right), the counter vortex is weaker than for the flat endwall and the secondary velocity vectors are also smaller, reducing the amount of fluid funneled into the inviscid core.

Finally, it can be noted that, at off-design incidence, the interaction between the passage and the counter vortices with the contoured endwall is stronger than at design incidence. The stronger interaction, along with the longer duration of the interaction due to lower secondary velocities, results in an increase in mixing, and therefore, in the mixing losses. For the flat endwall, the observation is the opposite. The interaction between the vortices is weaker at off-design incidence compared to design incidence. This weaker interaction is assumed to be due to the weaker vortices and the small change in size and magnitude between the two values of incidence compared to the contoured endwall cases. The weaker interaction observed at $7^{\circ}$ incidence will tend to reduce the mass-averaged total pressure loss coefficient and the mixing losses for the flat endwall compared to the contoured endwall, as will be shown in Section 5.2.3.4.

\subsubsection{Secondary Kinetic Energy}

The secondary kinetic energy (SKE), defined in Section 3.9.4.2, is the kinetic energy associated with the secondary velocity vectors in a plane perpendicular to the area-averaged

outlet flow angle, $\overline{\bar{\beta}}_{2}$. It is an indication of the magnitude of the secondary velocity vectors and of the strength of the cross-flow near the endwall. The secondary kinetic energy is ultimately a source of loss. The losses are generated through mixing as the flow moves downstream. Figure 5.5 shows contour lines of total pressure deficit coefficient overlaid with contour floods of the secondary kinetic energy for the flat (left) and contoured (right) endwall configurations at $7^{\circ}$ of incidence. The results at design incidence (Figure 4.7) have been repeated for comparison purposes. 
a) Design Incidence

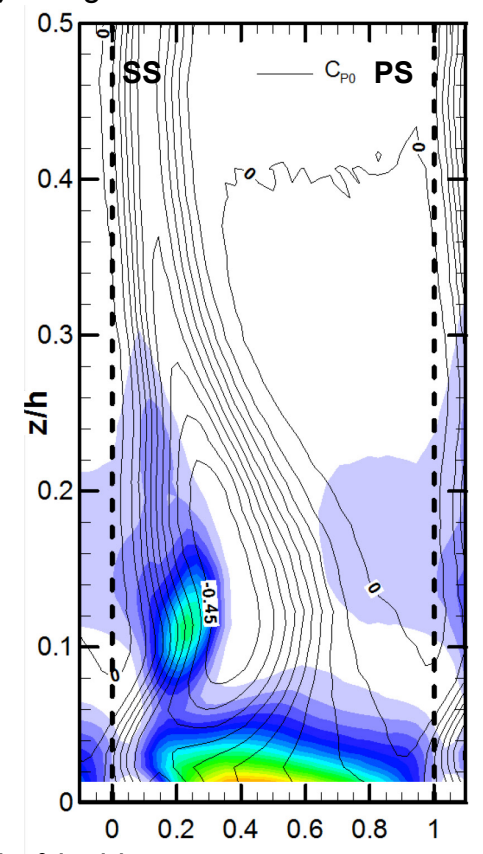

b) $7^{\circ}$ incidence

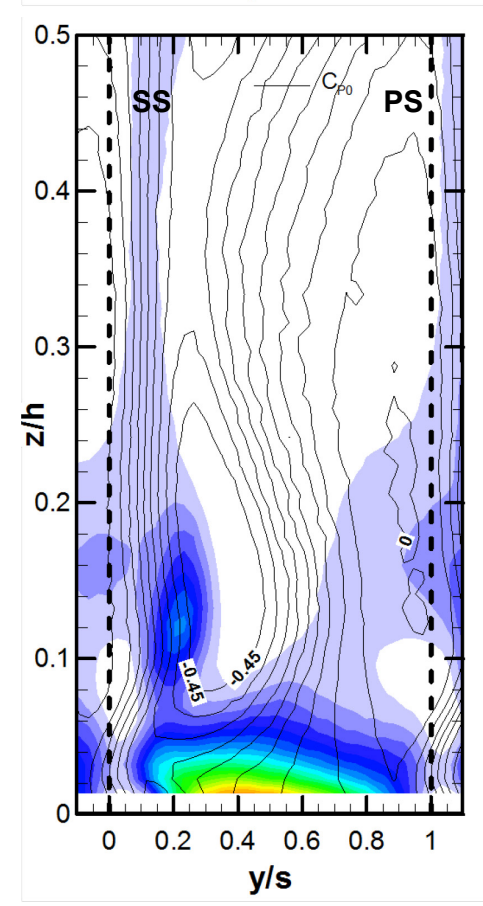

Contoured Endwall
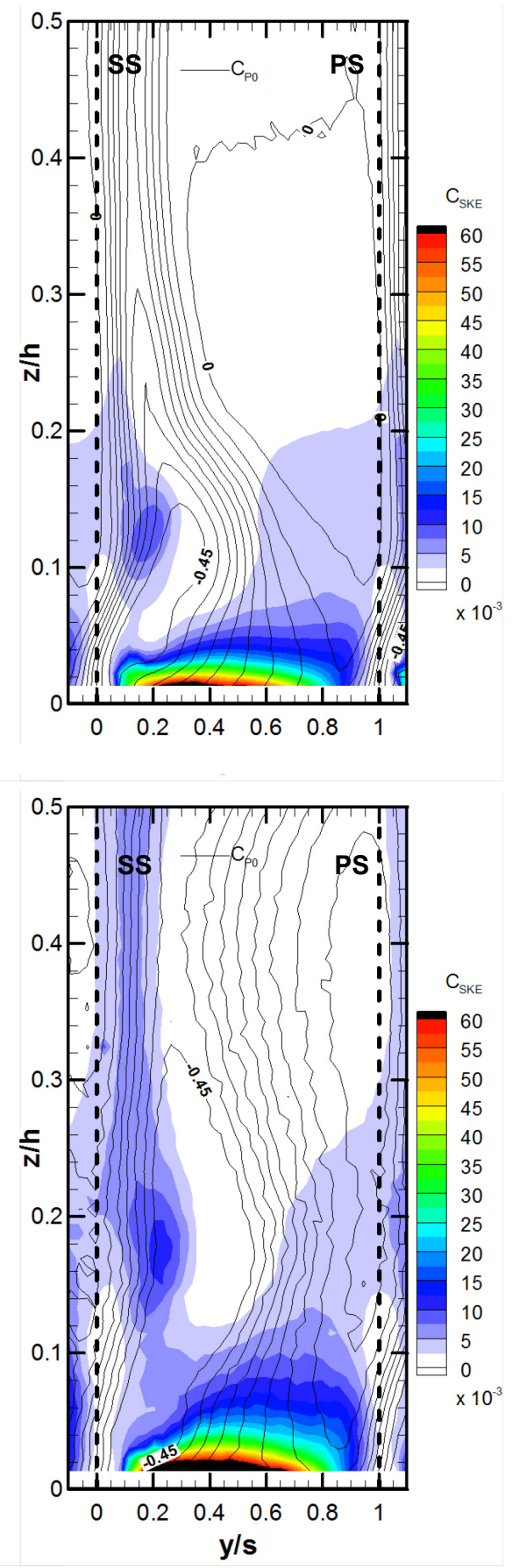

Figure 5.5: Line contours of total pressure deficit coefficient superimposed on flood contours of secondary kinetic energy for the flat (left) and contoured (right) endwall configurations at design (a) and $7^{\circ}$ (b) incidences 
As seen, there are two regions of fluid with relatively high secondary kinetic energy. The first region is located immediately adjacent to the endwall and corresponds to the flow overturning due to the cross-passage pressure gradient. For both endwall configurations, it occupies the majority of the passage near the endwall. The second region corresponds to the fluid moving off the endwall along the blade suction surface in the spanwise direction. This region is located between the passage and the counter vortices identified in the previous section. The secondary kinetic energy core is located at approximately $\mathrm{y} / \mathrm{s}=0.25$ and $\mathrm{z} / \mathrm{h}=$ 0.12 for flat endwall and $\mathrm{y} / \mathrm{s}=0.25$ and $\mathrm{z} / \mathrm{h}=0.17$ for the contoured endwall. It is also worth noting that, unlike the cases at design incidence, the second region of secondary kinetic energy expands up to midspan in the two-dimensional wake for both endwalls.

It is evident that, near the endwall, the peak level of secondary kinetic energy is higher for the contoured endwall than for the flat endwall. By comparing Figure 5.5 (b, right) to Figure 5.5 (a, right), it can be seen that the area occupied by the peak value of secondary kinetic energy coefficient for the contoured endwall is significantly larger at off-design incidence. However, for the flat endwall, the area occupied by the peak secondary kinetic energy is approximately the same between the two values of incidence. Moreover, for both endwall configurations, the first region of elevated secondary kinetic energy coefficient increases in size at off-design incidence. In the previous chapter, it was suggested that the higher secondary kinetic energy was directly due to the magnitude and strength of the vortical structures. However, as noted in the previous section, the passage vortex is weaker for both endwalls at off-design incidence, as defined by smaller peaks of streamwise vorticity coefficient. Thus, it is assumed that the larger secondary velocity vectors, and therefore the higher secondary kinetic energy, are mainly due to the increased size and magnitude of the vortical structures, instead of due to their strength.

By comparing the endwalls at $7^{\circ}$ of incidence (Figure 5.5 (b)), it appears that the higher secondary kinetic energy seen for the contoured endwall is directly due to the size, magnitude and strength of the vortical structures. In this case, it is assumed that the strength of the passage vortex is also a factor, as the peak magnitude of streamwise vorticity is slightly larger in this area for the contoured endwall. As shown earlier on Figure 5.4 (b), the passage vortex for the contoured endwall case is stronger towards the endwall across the blade 
passage. Thus, the induced secondary velocities and the corresponding secondary kinetic energy are expected to be higher for the contoured endwall. This can be observed on Figure 5.4 (b) and Figure 5.5 (b). The application of endwall contouring creates a stronger pitchwise pressure gradient, which results in generating higher secondary kinetic energy near the endwall. The stronger secondary kinetic energy will generate additional losses as it dissipates downstream and will possibly also generate higher viscous losses inside the endwall boundary within the passage due to higher wall shear. At design incidence, the mitigation of the corner stall offsets the additional losses due to the mixing out of the secondary kinetic energy and it resulted in overall loss reduction, as shown in Table 4.2. However, at $7^{\circ}$ of incidence, the mitigation of the corner stall by the application of endwall contouring does not appear to compensate for the additional losses resulting from the dissipation of the secondary kinetic energy and the resulting overall pressure losses are significantly larger than for the flat endwall, as it will be shown in Table 5.2.

The second region of high secondary kinetic energy corresponds to the flow driven up the suction surface of the blade by the secondary vortex and the subsequent interaction between the passage and counter vortices. The peak secondary kinetic energy coefficient for the flat endwall is approximately 1.33 times larger than the peak of the contoured endwall. The difference in peak magnitude is about half the difference observed at design incidence. By comparing the flat endwall contour plots of the design and off-design incidences as shown on Figure 5.5 (a, left) and Figure 5.5 (b, left), it can be seen that the size of the second region of high secondary kinetic energy is slightly smaller and the strength is noticeably smaller at off-design incidence. This result was expected based on the observations made on Figure 5.4 regarding the reduced strength of the secondary velocity vectors. The passage and counter vortices are weaker at off-design incidence and their interaction is also weaker, thus resulting in lower secondary kinetic energy. This observation suggests that the losses that will be generated from the mixing of the two vortices will be lower than at design incidence. For the contoured endwall, the observations are the opposite to the ones for the flat endwall. As the incidence increases, the size and magnitude of the peak secondary kinetic energy increase. This result was also expected due to the stronger interaction noted between the passage and counter vortices for the contoured endwall at off-design incidence observed on Figure 5.4 (b, 
right). The endwall contouring modifies the cross-passage flow to a different degree than for the design incidence such that the resulting passage vortex is modified. In this case, its overall magnitude and size increase significantly such that it results in a stronger interaction with the counter vortex and therefore in stronger secondary kinetic energy. The higher secondary kinetic energy will generate more losses as it mixes downstream. It can also be noted that the larger passage vortex pushes the counter vortex closer to midspan as evident by comparing the contoured endwall plots at both design and off-design incidences.

It is also worth noting that the peaks of secondary kinetic energy coefficients for both endwalls at off-design incidence are located within the core of the peak losses in the area where the interaction between the passage and counter vortices is the strongest, as shown on Figure $5.4(b)$.

As opposed to the design incidence cases, the stronger secondary kinetic energy near the endwall due to the application of endwall contouring results in larger overall total losses generated across the passage compared to the flat endwall test case, as observed in Table 5.2. Again, the endwall contouring having been optimized for the design inlet flow angle, at $7^{\circ}$ of incidence, the contouring is not effective and impacts the losses generated through the cascade. Therefore, these flow characteristics appear to show that the benefit resulting from the application of endwall contouring is only achieved over a narrow range of incidence angles near the design incidence.

\subsubsection{Integrated Downstream Results}

The contour floods presented in the previous section provide a qualitative understanding of the flow physics downstream of the blade row. A more quantitative comprehension can be obtained by looking at the pitchwise and overall mass- or areaaveraged parameters.

The pitchwise mass-averaged total pressure loss coefficient and secondary kinetic energy and the pitchwise area-averaged outlet flow angle measured at the downstream plane are shown on Figure 5.6 from the endwall to a specific spanwise position dependent of the endwall configuration. The overall mass- and area-averaged and the mixed-out massaveraged exit flow results are summarized in Table 5.2 along with the summary of the inlet 
experimental results repeated from Table 5.1. The results at design incidence, presented in Table 4.2, are also repeated for comparison purposes.

a) Mass-averaged total pressure loss coefficient

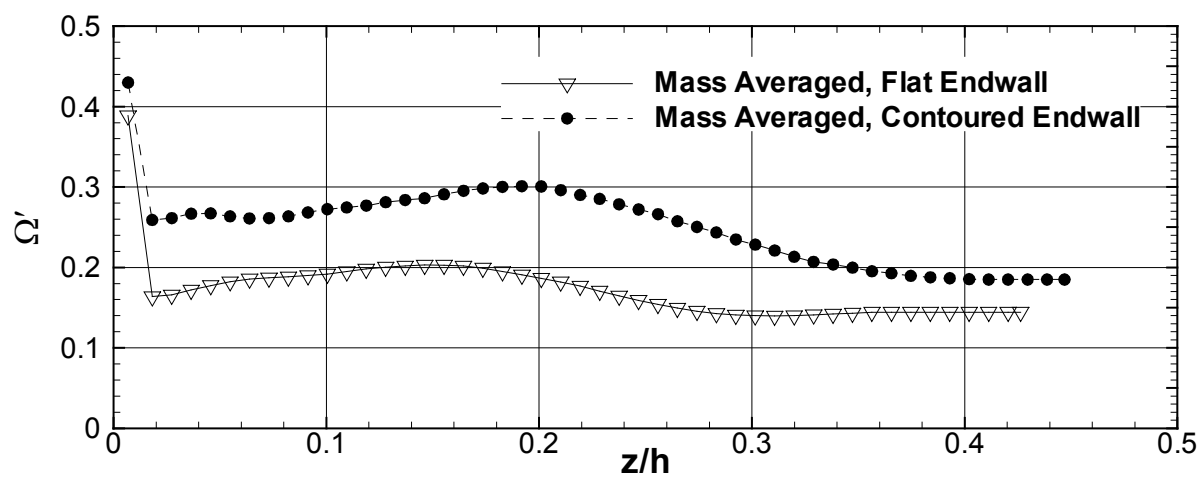

b) Area-averaged outlet flow angle

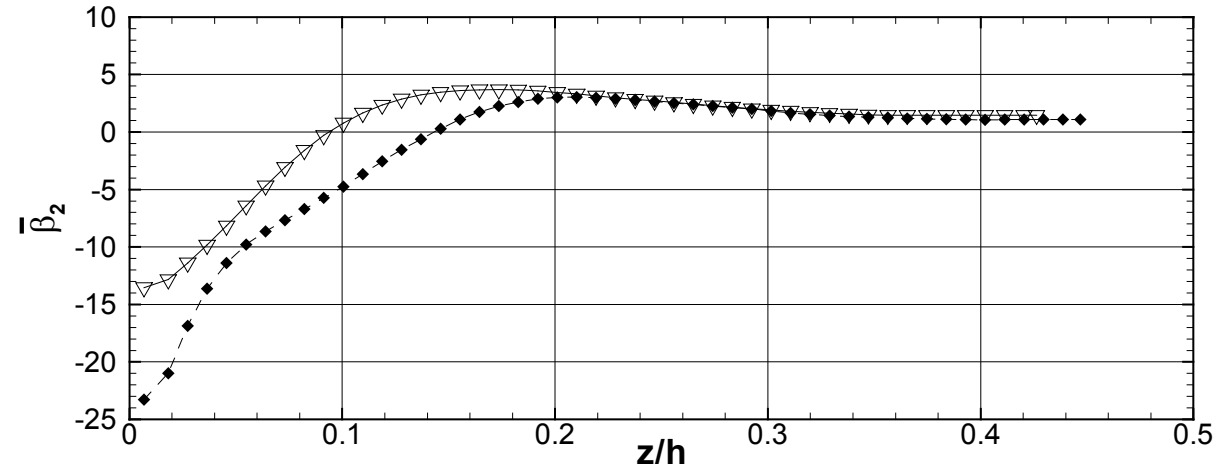

c) Mass-averaged secondary kinetic energy coefficient

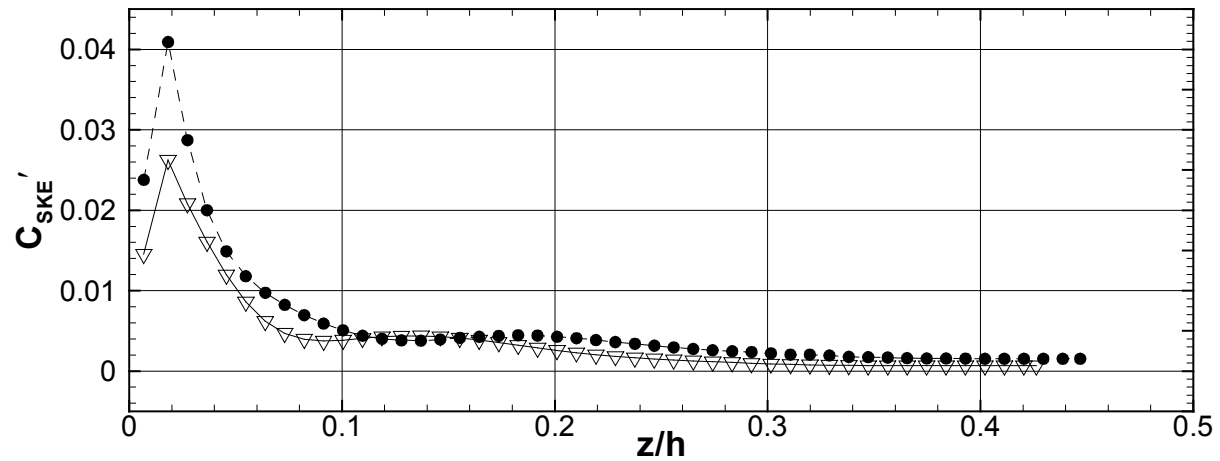

Figure 5.6: Measured pitchwise mass- and area-averaged results at $160 \% B_{x}$ plane: massaveraged total pressure loss coefficient (a), area-averaged outlet flow angle (b) and massaveraged secondary kinetic energy coefficient (c) at $7^{\circ}$ incidence 
Table 5.2: Summary of experimental results at 7 degrees of incidence

DESIGN INCIDENCE ENDWALL
70 INCIDENCE ENDWALL

\begin{tabular}{|c|c|c|c|c|c|}
\hline \multirow[b]{2}{*}{ LOCATION } & \multirow[b]{2}{*}{$\begin{array}{c}\text { FLOW } \\
\text { QUANTITY }\end{array}$} & \multicolumn{2}{|c|}{ 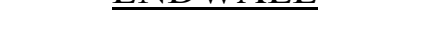 } & \multicolumn{2}{|c|}{$\bar{x}$} \\
\hline & & FLAT & CONTOURED & FLAT & CONTOURED \\
\hline \multirow[t]{7}{*}{ INLET } & $\delta / \mathrm{h}$ & 0.118 & 0.118 & 0.132 & 0.138 \\
\hline & $\delta^{*}[\mathrm{~mm}]$ & 2.57 & 2.25 & 2.83 & 2.70 \\
\hline & $\theta[\mathrm{mm}]$ & 1.99 & 1.80 & 2.22 & 2.15 \\
\hline & $\mathrm{H}$ & 1.29 & 1.25 & 1.27 & 1.26 \\
\hline & $\mathrm{C}_{\mathrm{q}, 1}{ }^{\prime \prime}$ & 0.982 & 0.987 & 0.985 & 0.973 \\
\hline & $\mathrm{C}_{\mathrm{q}, 1 \text { midspan }}{ }^{\prime}$ & 0.995 & 0.998 & 1.003 & 0.992 \\
\hline & $\mathrm{C}_{\mathrm{P} 0,1}{ }^{\prime \prime}$ & -0.011 & -0.010 & -0.021 & -0.022 \\
\hline OUTLET & $\mathrm{C}_{\mathrm{P} 0,2 \text { total }}{ }^{\prime \prime}$ & -0.089 & -0.077 & -0.171 & -0.244 \\
\hline \multirow[t]{11}{*}{$\mathrm{B}_{\mathrm{x}}=1.60$} & $\mathrm{C}_{\mathrm{P} 0,2 \text { profile }}{ }^{\prime}$ & -0.042 & -0.038 & -0.145 & -0.184 \\
\hline & $\mathrm{C}_{\mathrm{P} 0,2 \text { secondary }} "$ & -0.047 & -0.039 & -0.026 & -0.061 \\
\hline & $\Omega_{\text {total }}$ & 0.080 & 0.068 & 0.152 & 0.229 \\
\hline & $\Omega_{\text {profile }}$ & 0.041 & 0.038 & 0.144 & 0.187 \\
\hline & $\Omega_{\text {secondary }}$ & 0.039 & 0.030 & 0.008 & 0.042 \\
\hline & $\overline{\overline{\beta_{2}}}$ & -0.2 & -1.2 & 0.4 & -1.2 \\
\hline & $\overline{\bar{\beta}}_{2, \text { midspan }}$ & -1.3 & -1.3 & 1.5 & 1.1 \\
\hline & $\mathrm{C}_{\mathrm{q}, 2}{ }^{\prime \prime}$ & 0.506 & 0.492 & 0.478 & 0.441 \\
\hline & $\mathrm{C}_{\mathrm{q}, 2 \text { midspan }}{ }^{\prime}$ & 0.553 & 0.528 & 0.511 & 0.509 \\
\hline & $\mathrm{C}_{\mathrm{SKE}}{ }^{\prime \prime}$ & 0.003 & 0.002 & 0.004 & 0.005 \\
\hline & AVR & 1.106 & 1.070 & 1.228 & 1.217 \\
\hline \multirow[t]{10}{*}{$\begin{array}{l}\text { MIXED- } \\
\text { OUT }\end{array}$} & $\mathrm{C}_{\mathrm{P} 0, \text { total }}{ }^{\prime \prime}$ & -0.108 & -0.095 & -0.190 & -0.265 \\
\hline & $\mathrm{C}_{\mathrm{P} 0 \text {,profile }}{ }^{\prime}$ & -0.045 & -0.041 & -0.152 & -0.193 \\
\hline & $\mathrm{C}_{\mathrm{P} 0 \text {,secondary }}{ }^{\prime \prime}$ & -0.062 & -0.054 & -0.038 & -0.072 \\
\hline & $\Omega_{\text {total }}$ & 0.099 & 0.086 & 0.172 & 0.250 \\
\hline & $\Omega_{\text {profile }}$ & 0.045 & 0.041 & 0.152 & 0.196 \\
\hline & $\Omega_{\text {secondary }}$ & 0.054 & 0.045 & 0.020 & 0.054 \\
\hline & $\overline{\overline{\beta_{2}}}$ & -0.1 & -1.0 & 0.6 & -0.7 \\
\hline & $\overline{\bar{\beta}}_{2, \text { midspan }}$ & -1.2 & -1.3 & 1.5 & 1.1 \\
\hline & $\mathrm{C}_{\mathrm{q}}^{\prime \prime}$ & 0.453 & 0.436 & 0.427 & 0.378 \\
\hline & $\mathrm{C}_{\mathrm{q}, \text { midspan }}{ }^{\prime}$ & 0.542 & 0.519 & 0.493 & 0.487 \\
\hline
\end{tabular}


In addition, Figure 5.7 summarizes the measured and mixed-out losses results obtained at design incidence and at $7^{\circ}$ incidence for both flat and contoured endwalls.

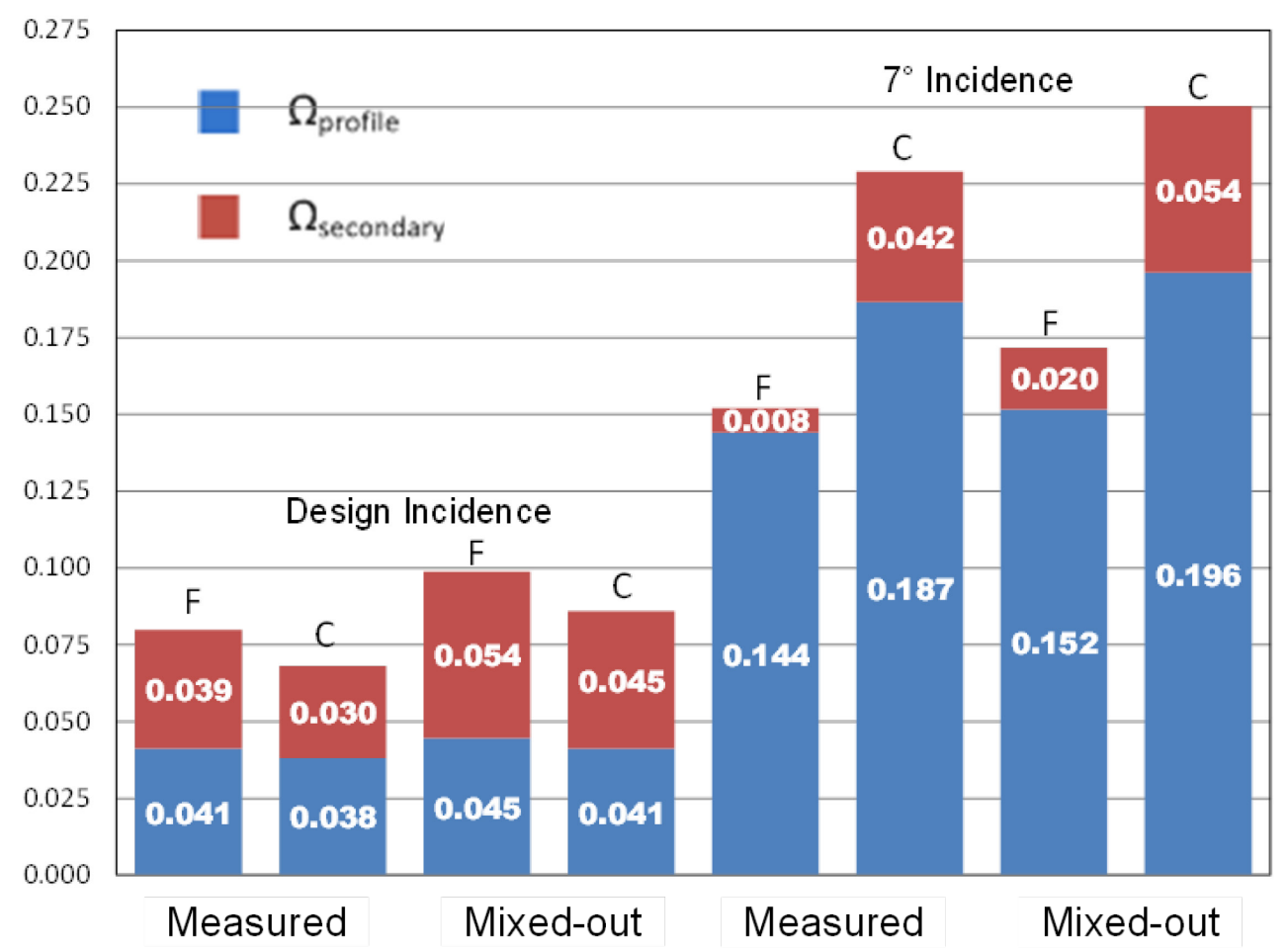

Figure 5.7: Summary of measured and mixed-out losses at design and $7^{\circ}$ incidences for the flat and contoured endwalls

In order to be able to calculate the mass-averaged parameters and compare the results of both endwalls, it was required that the integrated mass flow be approximately the same for each case. As mentioned in connection with Figure 5.2, the secondary flows were stronger on the opposite wall than on the one measured, creating larger blockage and forcing more mass flow to move through the measured half span. Since the blockage was different for each endwalls, it resulted in different integrated mass flows with an integration plane extending from the endwall to midspan. Therefore, in order to have the same mass flow, the integration limits in the spanwise direction had to be modified to a position below midspan. This can be observed on Figure 5.6 where the measured pitchwise integrated results are plotted to the spanwise location where the downstream integrated mass flow rate matched the inlet mass flow rate to midspan. 
As observed for the total pressure loss coefficient (Figure 5.6 (a)), the contouring produces higher pitchwise mass-averaged pressure losses for the whole span measured. This result is significantly different than at design incidence (see Figure 4.8 (a)), where the losses were reduced with contouring. However, an increase in losses was expected for the contoured endwall based on the observations made from the contour plots in the previous sections. The loss core and the two-dimensional wake were significantly larger in width and height for the contoured endwall compared to the flat endwall at off-design incidence. From the overall mass-averaged results presented in Table 5.2, the measured mass-averaged total pressure loss coefficient $\left(\Omega_{\text {total }}\right)$ and dynamic pressure loss coefficient $\left(\Omega_{\text {secondary }}\right)$ are 1.5 and 5.25 times larger for the contoured endwall than for the flat endwall, respectively. These results confirm that the application of endwall contouring has a significant impact in secondary loss production and that it does not appear to be beneficial as the incidence is increased.

A surprising result is the secondary losses for the flat endwall at off-design, which are significantly smaller than at design incidence, as shown in Table 5.2. This result was unexpected as the loss core was larger than at design incidence. In the current investigations at off-design incidence, the blade loadings and the larger wakes on the downstream measurement plane suggest that the boundary layers separate from the suction surface of the blade. The separations at midspan result in elevated profile losses, which are assumed to apply across the full span of the blade. Therefore, the current loss breakdown can lead to physically unrealistic values of secondary losses when there is significant separation at midspan. When these profile losses are subtracted from the measured total row losses to obtain the secondary losses, it can lead to secondary losses abnormally low or even negative, as observed in Table 5.2. However, the endwall flow could have affected the blade boundary layer such that to prevent separation or to re-attach the layer to the blade, thus reducing the effect of the flow separation in this region and resulting in a more realistic secondary loss value.

The pitchwise area-averaged outlet flow angle distribution at off-design incidence shown in Figure 5.6 (b) indicates that the blade row produces a typical over/underturning of the flow, similar to what was observed at design incidence and also by other investigators in both compressor such as Harvey (2008), Hoeger et al. (2002), Reising and Schiffer (2009a) 
and Sonoda et al. (2008). The application of contouring increases the overturning near the endwall by a maximum of $10^{\circ}$ compared with the flat endwall case. This is approximately twice as much as at design incidence and may be explained by the different effects of contouring at off-design incidence, as discussed in the previous sections. Unlike at design incidence (see Figure 4.8 (b)), the flow remains overturned up to a higher spanwise position, which is due to the stronger secondary velocity vectors observed at off-design incidence. The above observations are consistent with the inferences made from the contour floods presented in the previous sections. As shown on Figure 5.4 (b), near the endwall, the secondary flow velocity vectors are stronger for the contoured endwall due to the increase strength of the cross-passage pressure gradient. The stronger pressure gradient results in increasing the overturning of the flow compared to the flat endwall. The weaker interaction between the passage and counter vortices resulting from the application of contouring decreases slightly the underturning compared to the flat endwall as observed on Figure 5.6. It is also worth noting that, unlike at design incidence, the effect of endwall contouring on the area-averaged outlet flow angle seems to be significantly reduced closer to the endwall. Starting slightly above $\mathrm{z} / \mathrm{h}=0.20$, the flow appears to be unaffected by the application of contouring, compared to design incidence where it extends to about $\mathrm{z} / \mathrm{h}=0.4$. This could be a result of the two-dimensional region being pushed away from midspan by the stronger secondary flows of the opposite endwall observed on Figure 5.2. The area-averaged outlet flow angles at midspan for both endwall cases at $7^{\circ}$ of incidence are slightly underturned, unlike at design incidence where a small overturning was observed. This underturning would suggest that the flow is not diffused as much as at design incidence. As shown in Table 5.2, the overall areaaveraged outlet flow angle of the contoured endwall is overturned to the same extent as at design incidence. The overall area-averaged outlet flow angle of the flat endwall is slightly underturned, unlike at design incidence where it was overturned.

Figure 5.6 (c) shows the pitchwise mass-averaged secondary kinetic energy. The higher secondary kinetic energy near the wall for the contouring is a result of the flow overturning shown on Figure 5.6 (b) and will eventually cause additional pressure losses through mixing. However, unlike at design incidence where the stronger cross-flow delays the corner stall and results in a reduction in losses, the increase in strength of the cross-flow at $7^{\circ}$ of incidence 
does not provide the same benefit. As discussed above and shown on Figure 5.6 (a), the pitchwise mass-averaged total pressure loss coefficient of the contoured endwall case remains larger than the flat endwall for the whole span. A weak second peak of secondary kinetic energy is observed around $\mathrm{z} / \mathrm{h}=0.14$ and $\mathrm{z} / \mathrm{h}=0.19$ for the flat and contoured endwalls, respectively. As for the design incidence experiment, the second peak corresponds to the flow underturning and to the spanwise flow being funnelled into the low-loss region. The difference in spanwise position of the second peaks was expected. Because of the larger passage vortex of the contoured endwall, the counter vortex is pushed closer to midspan. Thus, the interaction between the two vortices, main source of the flow underturning and of the second peak of secondary kinetic energy, is also pushed further towards midspan. Unlike at design incidence, the application of endwall contouring resulted in higher mass-averaged secondary kinetic energy at all spanwise locations except for a small region from $\mathrm{z} / \mathrm{h}=0.12$ to 0.15 . This region corresponds to the second peak of secondary kinetic energy of the flat endwall. Overall, the measured mass-averaged $C_{S K E}$ for the contoured endwall is 1.25 times larger than for the flat endwall at off-design incidence and 2.25 times larger than for the contoured endwall at design incidence.

The overall mixed-out mass-averaged exit flow results are shown in Table 5.2. As discussed in Chapter 3, the mixed-out losses are calculated from a control volume that extends at constant area from the downstream measurement plane to a hypothetical mixedout plane where uniform conditions exist. On this hypothetical plane, the secondary kinetic energy is completely dissipated and appears as total pressure losses. In addition to the dissipation of the secondary kinetic energy, mixing losses are generated through the recovery of static pressure and the mixing-out of the non-uniformities in the primary kinetic energy. The mixed-out mass-averaged total pressure loss coefficient $\left(\Omega_{\text {total }}\right)$ and the dynamic pressure loss coefficient $\left(\Omega_{\text {secondary }}\right)$ are 1.4 and 2.7 times larger for the contoured endwall than for the flat endwall case, respectively. The mixed-out area-averaged outlet flow angle is slightly underturned for the flat endwall, whereas it was overturned at the measurement plane. The outlet flow angle is slightly less overturned for the contoured endwall when compared to the measurement plane. 
Figure 5.8 shows the influence of incidence on the overall measured and mixed-out total-pressure loss coefficients. Since measurements were taken at only two different values of incidence, it was assumed that the losses increased linearly with incidence. As the incidence increases, the measured and mixed-out losses of the contoured endwalls increase more rapidly, such that at $7^{\circ}$ incidence, the losses are approximately 1.5 times larger than for the flat endwall, compared to 0.85 times the losses at design incidence. Also, from the tabulated results and Figure 5.8, it can be seen that the overall measured total-pressure losses at $7^{\circ}$ incidence are 1.9 and 3.4 times the values at design incidence for the flat and contoured endwalls, respectively. It is clearly seen that the losses increase more rapidly with the contoured endwall than with the flat endwall. Another interesting observation is that, assuming that the relationship is linear, the application of endwall contouring only provides a net benefit up to about $1^{\circ}$ of incidence. These results suggest that the application of endwall contouring for highly-loaded compressors may only be beneficial for blade rows that experience a narrow range of incidence values in service. Therefore, further investigations performed at inlet flow incidences between the two tested values would provide a clearer idea of the relationship between losses and incidence.

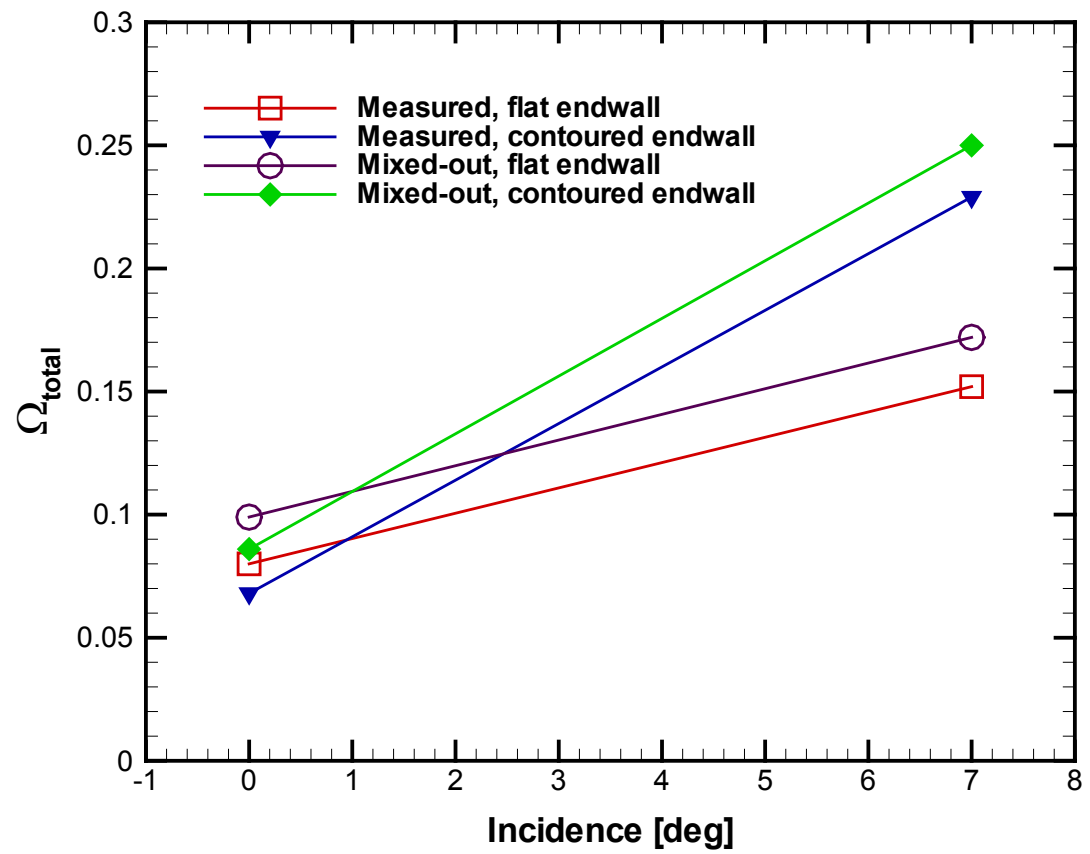

Figure 5.8: Influence of incidence on measured and mixed-out total-pressure losses for the flat and contoured endwalls 


\subsection{Summary of Results and Conclusions}

The objectives of this chapter were to investigate the relationship between secondary losses and incidence and the influence of endwall contouring on secondary losses at offdesign incidence. Experimental results were presented for the flow field of a compressor exit guide vane with and without the application of non-axisymmetric endwall contouring at $7^{\circ}$ of positive incidence. The measurements were compared with the corresponding results obtained at design incidence.

The airfoil surface static pressure measurements showed that the pressure distributions at all measured spanwise locations were slightly more front-loaded at higher incidence. Also, a region of constant pressure was observed at midspan from the middle of the blade up to the trailing edge, suggesting a breakdown in diffusion and presumably flow separation. This is a probable explanation to the large increase in profile losses at the off-design value of incidence, as observed in the present chapter.

Endwall contouring increases the intensity of the cross-passage flow near the endwall in order to drive the endwall flow towards the suction surface/endwall corner in order to reduce or delay the formation of the corner stall. It was found that, at design incidence, the mitigation of the corner stall by the application of contouring did provide a net benefit in secondary losses reduction. However, the manipulation of the contouring did not provide comparable benefits at off-design incidence. In this case, the secondary kinetic energy, the loss core and the size of the passage vortex all increased significantly, resulting in an increased in the integrated secondary losses. Thus, the application of endwall contouring resulted in the opposite of the desired goal to reduce the secondary losses.

Therefore, it appears that endwall contouring, which must be optimized for a particular value of incidence, is only suitable for highly-loaded compressor blade rows that experience a narrow range of incidence values. 


\section{Chapter 6}

\section{Conclusions and Recommendations for Future Work}

\subsection{Conclusions}

The thesis investigated the losses associated with the secondary flows in a highlyloaded compressor blade row. This research was conducted as part of a project with Pratt \& Whitney Aircraft in order to obtain experimental data which would be used to validate their CFD models. It investigated the effects of non-asymmetric endwall contouring on the secondary flows and their associated losses. The influence of incidence was also investigated for both the nominal and contoured endwalls. This section presents the overall conclusions of the present study. More detailed conclusions for each of the specific topics were presented in Chapter 4 and Chapter 5.

Flow measurements were initially done with the blades installed on a baseline flat endwall and at the design inlet flow incidence for comparison with the contoured endwall results. The measurements showed that the secondary losses accounted for approximately $50 \%$ of the total losses.

Subsequently, it was found that the application of non-axisymmetric endwall contouring increased the intensity of the cross-passage flow near the endwall. This resulted in more high momentum fluid being convected towards the suction surface/endwall corner and up the blade span. The investigations showed that the flow was more overturned near the endwall with the application of contouring compared to the flat endwall test case. This resulted in higher secondary kinetic energy (SKE), and ultimately higher losses, being 
generated near the endwall. Although increasing the strength of the cross-passage flow increased the strength of the passage vortex and increased the secondary losses near the endwall, it was concluded that delaying the formation of the corner stall was more beneficial and offset these losses. As observed from the pitchwise averaged plots, the losses and the SKE were larger near the endwall for the contoured endwall test case, mainly due to the more overturned flow. However, the losses and the SKE rapidly became smaller than the flat endwall values away from the endwall towards midspan. Additionally, in the high loss region, where both the passage and counter vortices interact with each other, the flow was less underturned for the profiled endwall than for the flat endwall. The overall mass-averaged secondary losses across the passage were reduced by more than $20 \%$ when compared to the flat endwall results and accounted for approximately $44 \%$ of the total losses. Additionally, the mass-averaged secondary kinetic energy at the outlet plane was reduced by approximately $33 \%$, therefore contributing in less losses being generated as this energy dissipated until the flow was fully mixed-out.

The oil surface flow visualisations provided a qualitative interpretation of the threedimensional flow field near the walls of the blade passage and gave insights into how the flow is affected by the application of endwall contouring. It was found that the contouring does not change the broad physics of the flow. However, some differences were noted. The cross-flow for the contoured endwall case was more perpendicular to the suction surface of the blade compared to the flat endwall, as expected from the more overturned flow near the endwall observed from the measurements made downstream of the passage. This stronger cross-flow was a result of the higher pitchwise pressure gradient, induced by curvature of the contoured endwall. Additionally, it was observed that the contoured endwall reduced the extent of the corner separation on the endwall and on the suction surface of the blade. This was mainly due to the stronger cross-passage pressure gradient driving the secondary flows into the suction corner and up the blade surface, modifying their interaction with the suction surface boundary layer and therefore, reducing the corner stall. The smaller corner stall resulted in lower losses, as confirmed by the pressure probe measurements.

Detailed flow measurements were also obtained with the blade row at $+7^{\circ}$ of incidence. This investigation was performed on both endwall configurations in order to 
understand the effects of positive incidence and the resulting higher aerodynamic loading on the flow field. It was found that the total losses generated for the flat and contoured endwalls were approximately 1.9 and 3.4 times larger, respectively, than at design incidence. This significant increase in loss was mainly due to the increase in the profile losses (i.e. approximately 3.5 and 4.9 times larger for the flat and contoured endwalls, respectively). At off-design incidence, the flow separated from the blade suction surface and did not re-attach, causing a breakdown in diffusion and a significant increase in the profile losses. The difference in the profiles losses between the two endwall cases is an indication that the flows are not two-dimensional at midspan and that the deviation from two-dimensionality differs between the two cases. Therefore, this observation indicates that the endwall flows are influencing the midspan flow to different degrees for the two cases.

It was also found that the application of endwall contouring resulted in higher secondary losses at the positive incidence, approximately 1.4 times larger than at design incidence. The cross-flow on the contoured endwall was stronger and more overturned compared to the flat endwall test case, as observed at design incidence. This resulted in higher secondary kinetic energy being generated near the endwall. However, in this case, the streamwise vorticity and secondary kinetic energy penetrated deeper along the span and occupied a larger area, resulting in a higher mass-averaged secondary kinetic energy coefficient when compared to the flat endwall test case. This additional secondary kinetic energy contributed to the higher losses generated as it dissipated downstream of the cascade, as confirmed by the higher mixed-out total and secondary losses. Additionally, the increased penetration depth of the secondary flows at positive incidence influenced the midspan flow to different degrees than for the flat endwall case, resulting in higher profile losses. Therefore, at the positive incidence investigated, the application of endwall contouring did not show the benefits observed at the design incidence. Additional total and secondary losses were generated with the profiled endwall when compared to the flat endwall.

The current research showed that the application of endwall contouring could be used as a means of secondary and total losses reduction across a highly-loaded compressor blade stage. The study at design incidence showed benefits with endwall contouring as the stronger cross-flow resulted in mitigating the corner stall and reducing the losses. However, these 
benefits were not observed at the positive incidence studied. Additional measurements at values of incidence between the two values tested would be required to provide a better assessment of the benefits of contouring at off-design values of incidence. In addition, contouring adds complexity to the design of a compressor row, potentially resulting in additional manufacturing and maintenance cost. Thus, engine designers would need to evaluate if the benefits observed are worth the additional cost.

\subsection{Recommendations for Future Work}

The current research provided a good understanding of the development of the secondary flow structures across a compressor linear cascade and on their associated losses at both design and positive values of incidence. However, some additional investigations could be conducted to improve the understanding of the flow mechanisms.

The current loss breakdown can lead to physically unrealistic values of secondary losses when there is significant separation at midspan. The separation at midspan will result in elevated profile losses, which are assumed to apply across the full span of the blade. When these profile losses are subtracted from the measured total row losses to obtain the secondary losses, it can lead to secondary losses abnormally low or even negative, as observed for the flat endwall case at positive incidence. However, the endwall flow could have affected the blade boundary layer such that to prevent separation or to re-attach the layer to the blade, thus reducing the effect of the flow separation in this region. The present author believes that investigations using a different loss breakdown, such as the one proposed by Benner (2003), which considers the penetration depth of the secondary flows and removes it from the profile loss calculation, would allow to interpret the secondary flows and their associated losses with more accuracy.

The surface flow visualisations provided a detailed interpretation of the threedimensional flow field within the blade passage, but were only conducted at the cascade design inlet incidence. The present author believes that flow visualisations at off-design incidences could be used to develop a better understanding of the flow physics within the blade passage in these conditions and would support the interpretation of the pressure probe results. It could provide information regarding the development of the secondary flow 
structures at off-design incidences and how it differs from the design incidence. Additionally, it could be used to confirm the presence of midspan boundary layer separation on the suction surface of the CEGV-1 compressor blade that was inferred from the blade loading distributions.

As discussed, the application of endwall contouring resulted in higher secondary losses being generated across the blade passage at positive incidence compared to the flat endwall case. Flow visualisation on the contoured endwall at positive incidence could provide information on how the flow is affected by the concave and convex curvatures. The endwall contouring at positive incidence might produce local separations on the endwall, which would generate additional losses locally and therefore, reduce the net benefit from the contouring. These local separations would not be apparent in the probe measurements, but might be revealed by the flow visualisation. Therefore, the author believes that the visualisation of the flow mechanisms within the passage on the endwall and on the surfaces of the blade would improve the understanding of the physics and could help the engine manufacturers to improve the design of the profiled endwall and potentially increase its benefits.

From the current study, it was shown that endwall contouring showed benefits at the design inlet flow incidence, but that, at the positive incidence investigated $\left(+7^{\circ}\right)$, the same benefits were not observed and additional losses were generated. A potential avenue would be to investigate the effect of endwall contouring at intermediate inlet flow incidences between the two incidences studied in order to have more data for the curves shown on Figure 5.8 and to see the extent of the benefits obtained from endwall contouring. Furthermore, as discussed previously, the profiled endwall design was optimized for the blade design incidence. The design of a slightly less aggressive profiled endwall than the current one could potentially show benefits over a larger range of engine operating conditions. The performance might be slightly reduced at the design incidence, but the profiled endwall design would be more robust if its benefits were relatively insensitive to changes in conditions such as incidence, Mach number and Reynolds number.

Finally, an additional avenue that could be investigated is the combination of endwall contouring with suction or blowing flow control. This type of flow control could be used to 
mitigate the size and strength of the inlet boundary layer, leading to a potential reduction in the horseshoe vortex strength and therefore, in the losses that will be generated across the passage. Additionally, suction could be used to mitigate the corner stall. In her literature review, Ellison (2005) commented that many investigations on flow control via suction or blowing in the area of the corner stall did not show any benefits. However, corner suction upstream of the start of the corner separation might be useful in delaying the start of the corner stall to a point further downstream. Also, the use of selective suction on the endwall might be beneficial by allowing larger pressure gradients without causing separation of the endwall boundary layer or by increasing the diffusion across the passage. However, the benefits of the suction in reducing the losses would have to be weighed against the pumping power that might have to be provided to obtain the suction. The present investigation showed some potential benefits on corner stall mitigation and secondary losses reduction with the use of endwall contouring. Therefore, a combination of aspiration flow control to delay the start of the corner stall and increase diffusion and of endwall contouring to mitigate the corner stall could be an area of research worth pursuing. However, this method of flow control adds complexity to the design, which would potentially result in higher manufacturing and maintenance cost. Therefore, a trade-off between the gains obtained from this method of loss reduction and the additional cost generated would need to be conducted by the engine manufacturers. 


\section{References}

Abo El Ella, H. M. and Sjolander, S. A., 2012, “An Application of a Low Cost High Contrast Oil Flow Visualisation Technique in a Transonic Blow-Down Wind Tunnel for the Investigation of Secondary Flows," ASME Paper No. GT2012-68653, Copenhagen, Denmark, June 11-15.

Adkins, G. G. and Smith, L. H., 1982, "Spanwise Mixing in Axial-Flow Turbomachines," ASME Journal of Eng. Power., 104, pp. 97.

Aungier, R. H., 2003, "Axial-Flow Compressors: A Strategy for Aerodynamic Design and Analysis,” ASME Press, pp. 361.

Benner, M. W., 2003, "The Effects of Leading Edge Geometry on Profile and Secondary Losses in Turbine Cascades," PhD thesis, Carleton University, Ottawa, Canada.

Benner, M. W., Sjolander, S. A. and Moustapha, S. H., 1997, "Measurements of Secondary Flows in a Turbine Cascade at Off-Design Incidence," Proceedings of the 1997 International Gas Turbine and Aeroengine Congress and Exposition Paper No. 97-GT-382, Orlando, FL, USA, June 2 - June 5.

Benner, M. W., Sjolander, S. A. and Moustapha, S. H., 2004, "The Influence of Leading-Edge Geometry on Secondary Losses in a Turbine Cascade at the Design Incidence," ASME Journal of Turbomachinery, 126, pp. 277-287.

Blight, F. G. and Howard, W., 1952, "Deflection, Drag, and Velocity Distributions," Dept. Supply, A. R. L., Reps. E.74, Melbourne, Australia.

Bullock, R. O. and Johnsen, I. A., 1965, "Aerodynamic Design of Axial Flow Compressors," NASA, L. R. C., NASA SP-36, Cleveland, OH, United States of America.

Choi, M., Oh, S. H., Ko, H. Y. and Baek, J. H., 2008, "Effects of the Inlet Boundary Layer Thickness on Rotating Stall in an Axial Compressor," ASME Paper No. GT2008-50886, Berlin, Germany, June 9-13.

Chue, S. H., 1975, "Pressure Probes for Fluid Measurement," Progress in Aerospace Sciences, 16, pp. 147-223.

Cumpsty, N. A., 2004, Compressor Aerodynamics, Second Edition, Krieger Publishing Company.

Denton, J. D., 1993, “The 1993 Igti Scholar Lecture: Loss Mechanisms in Turbomachines," ASME Journal of Turbomachinery, 115, pp. 621-656. 
Dickens, T. and Day, I., 2009, "The Design of Highly Loaded Axial Compressors," ASME Paper No. GT-2009-59291, Orlando, Florida, USA, June 8-12.

Dorfner, C., Nicke, E. and Voss, C., 2007, "Axis-Asymmetric Profiled Endwall Design Using Multiobjective Optimization Linked with 3d Rans-Flow-Simulations," ASME Paper No. GT2007-27268, Montreal, Qc, Canada, May 14-17.

Douglas, J. W., Li, S. M., Song, B., W.F., N., Sonoda, T. and Arima, T., 2003, "Effects of Freestream Turbulence on the Losses of a Highly-Loaded Compressor Stator Blade," ASME Paper No. GT-2003-38604, Atlanta, Georgia, USA, June 16-19.

Drela, M. and Youngren, H., 1991, "Viscous/Inviscid Method for Preliminary Design of Transonic Cascades," AIAA Journal, pp. 675-679.

Ellison, T. A., 2005, "Investigation of a Concept for Highly-Loaded Axial-Compressor Stators Using Flow Control," Final Report - Research Agreement No. 983061, Compressor Aerodynamics, Pratt \& Whitney Canada, Ottawa, Canada.

Gallimore, S. J. and Cumpsty, N. A., 1986, "Spanwise Mixing in Multistage Axial Flow Compressors: Part I - Experimental Investigation,” ASME Journal of Turbomachinery, 108, pp. 2.

Gallington, R. W., 1981, "Measurement of Very Large Flow Angles with Non-Nulling SevenHole Probes," Proceedings of the 27th International Instrumentation Symposium, Indianapolis, IN, USA, April 27-30.

Gbadebo, S. A., Cumpsty, N. A. and Hynes, T. P., 2004, "Three-Dimensional Separations in Axial Compressors,” ASME Paper No. GT2004-53617, Vienna, Austria, June 14-17.

Gerner, A. A., Maurer, C. L. and Gallington, R. W., 1984, "Non-Nulling Seven-Hole Probes for High Angle Flow Measurement," Experiments in Fluids, 2, pp. 95-103.

Goobie, S. M., 1989, "A Test Section for Investigation the Off-Design Performance of Planar Cascades," MASc thesis, Carleton University, Ottawa, Canada.

Goodhand, M. N. and Miller, R. J., 2010, "The Impact of Real Geometries on Three-Dimensional Separations in Compressors," ASME Paper No. GT-2010-22246, Glasgow, UK, June 1418.

Gregory-Smith, D. G. and Cleak, J. G. E., 1992, "Secondary Flow Measurements in a Turbine Cascade with High Inlet Turbulence," ASME Journal of Turbomachinery, 114, pp. 173183.

Gregory-Smith, D. G., Graves, C. P. and Walsh, J. A., 1988, "Growth of Secondary Losses and Vorticity in an Axial Turbine Cascade," ASME Journal of Turbomachinery, 110, pp. 1-8. 
Hartland, J. C., Gregory-Smith, D. G., Harvey, N. W. and Rose, M. G., 2000, "Nonaxisymmetric Turbine End Wall Design: Part Ii- Experimental Validation," ASME Journal of Turbomachinery, 122, pp. 286-293.

Harvey, N. W., 2008, "Some Effects of Non-Axisymmetric End Wall Profiling on Axial Flow Compressor Aerodynamics. Part I: Linear Cascade Investigation," ASME Paper No. GT2008-50990, Berlin, Germany, June 9-13.

Harvey, N. W. and Offord, T. P., 2008, "Some Effects of Non-Axisymmetric End Wall Profiling on Axial Flow Compressor Aerodynamics. Part Ii: Mulit-Stage Hpc Cfd Study," ASME Paper No. GT-2008-50991, Berlin, Germany, June 9-13.

Harvey, N. W., Rose, M. G., Taylor, M. D., Shahpar, S., Hartland, J. and Gregory-Smith, D. G., 2000, "Nonaxisymmetric Turbine End Wall Design: Part I- Three-Dimensional Linear Design System," ASME Journal of Turbomachinery, 122, pp. 278-285.

Hergt, A., Meyer, R., Liesner, K. and Nicke, E., 2011, "A New Approach for Compressor Endwall Contouring,” ASME Paper No. GT-2011-45858, Vancouver, Canada, June 6-10.

Herrig, L. J., Emery, J. C. and Erwin, J. R., 1957, "Systematic Two-Dimensional Cascade Tests of Naca 65-Series Compressor Blades at Low Speeds," NASA, NACA TN 3916,

Hodson, H. P. and Dominy, R. G., 1987, "Three-Dimensional Flow in a Low-Pressure Turbine Cascade at Its Design Condition,” ASME Journal of Turbomachinery, 109, pp. 177-185.

Hoeger, M., Cardamone, P. and Fottner, L., 2002, "Influence of Endwall Contouring on the Transonic Flow in a Compressor Blade," ASME Paper No. GT-2002-30440, Amsterdam, Netherlands, June 3-6.

Howell, A. R., 1945, "The Design of Axial Flow Compressors," Proceedings of the Institution of Mechanical Engineers W.E.P. No. 12, 153.

Kang, S. and Hirsch, C., 1991, "Three Dimensional Flow in a Linear Compressor Cascade at Design Conditions," Internation Gas Turbine and Aeroengine Congress and Exposition Paper No. 91-GT-114, Orlando, Florida, June 3-6.

Knezevici, D. C., 2011, "Controlling Secondary Flows in Very Highly Loaded Low-Pressure Turbine Cascades," PhD thesis, Carleton University, Ottawa, Canada.

Knezevici, D. C., Sjolander, S. A., Praisner, T. J., Allen-Bradley, E. and Grover, E. A., 2008, "Measurements of Secondary Losses in a Turbine Cascade with the Implementation of Non-Axisymmetric Endwall Contouring," ASME Paper No. GT2008-51311, Berlin, Germany, June 9-13.

Knezevici, D. C., Sjolander, S. A., Praisner, T. J., Allen-Bradley, E. and Grover, E. A., 2010, "Measurements of Secondary Losses in a Turbine Cascade with the Implementation of Nonaxisymmetric Endwall Contouring," ASME Journal of Turbomachinery, 132, pp. 011013. 
Koch, C. C. and Smith, L. H., 1976, "Loss Sources and Magnitudes in Axial-Flow Compressors," ASME J. Engineering for Power, 98, pp. 411-424.

Köller, U., Mönig, R., Küsters, B. and Schreiber, H.-A., 2000, "Development of Advanced Compressor Airfoils for Heavy-Duty Gas Turbines, Part I: Design and Optimization," ASME Journal of Turbomachinery, 122, pp. 397-405.

Konig, W. M., Hennecke, D. K. and Fottner, L., 1996, "Improved Blade Profile Loss and Deviation Models for Advanced Transonic Compressor Bladins: Part I - a Model for Subsonic Flow," ASME Journal of Turbomachinery, 118, pp. 73-80.

Kozulovic, D., Stark, U. and Lewin, E., 2009, "Hub-Corner Stall in Compressor Cascades: A Comparison between Experimental and Numerical Results," $19^{\text {th }}$ ISABE Conference Paper No. ISABE-2009-1335, Montreal, QC, CA, Sept. 11-17.

Kröger, G., VoB, C., Nicke, E. and Cornelius, C., 2011, "Theory and Application of Axisymmetric Endwall Contouring for Compressors," ASME Paper No. GT2011-45624, Vancouver, BC, Canada, June 6-10.

Küsters, B., Schreiber, H.-A., Köller, U. and Mönig, R., 1999, "Development of Advanced Compressor Airfoils for Heavy-Duty Gas Turbines, Part Ii: Experimental and Analytical Analysis," ASME Journal of Turbomachinery, 122, pp. 406-414.

Lakshminarayana, B., 1996, Fluid Dynamics and Heat Transfer of Turbomachinery, John Wiley \& Sons, Inc.

Lei, V.-M., Spakovszky, Z. S. and Greitzer, E. M., 2008, “A Criterion for Axial Compressor Hub-Corner Stall,” ASME Journal of Turbomachinery, 130, pp. 031006-1-10.

Lepot, I., Mengistu, T., Hiernaux, S. and De Vriendt, O., 2011, "Highly Loaded Lpc Blade and Non Axisymmetric Hub Profiling Optimization for Enhanced Efficiency and Stability," ASME Paper No. GT-2011-46261, Vancouver, Canada, June 6-10.

Lewis, R. I. and Hill, J. M., 1971, "The Influence of Sweep and Dihedral in Turbomachinery Blade Rows," Journal of Mechanical Engineering Science, 13, pp. 266-285.

Lewis, W. E., 1966, "Fixed-Direction Probes for Aerodynamic Measurements," Proceedings Institution of Mechanical Engineers 1965-66, 180 Part 3J, pp. 141-151.

Lieblein, S., Schwenk, F. C. and Broderick, R. L., 1953, "Diffusion Factor for Estimating Losses and Limiting Blade Loadings in Axial-Flow-Compressor Blade Elements," NACA, RM E53D01, Washington, United States of America.

Lu, J., Chu, W. and Wu, Y., 2009, "Effects of Endwall Profiling on Axial Flow Compressor Stage,” ASME Paper No. GT-2009-59418, Orlando, Florida, USA, June 8-12. 
Ma, W., Ottavy, X., Lu, L., Leboeuf, F. and Gao, F., 2011, "Experimental Investigations of Corner Stall in a Linear Compressor Cascade," ASME Paper No. GT-2011-45236, Vancouver, CAN, June 6-10.

MacIsaac, G. D., Sjolander, S. A. and Praisner, T. J., 2010, "Measurements of Losses and Reynolds Stresses in the Secondary Flow Downstream of a Low-Speed Linear Turbine Cascade," ASME Paper No. GT-2010-22727, Glasgow, UK, June 14-18.

MacIsaac, G. D., Taremi, F., Knezevici, D. C., Scribner, C. A. and Sjolander, S. A., 2009, "Challenges in Using Kiel and Seven-Hole Pressure Probes in Highly Three-Dimensional Flows: Application to Turbomachinery Cascade Measurements," $19^{\text {th }}$ ISABE Conference Paper No. ISABE-2009-1209, Montreal, QC, CA, Sept. 11-17.

Mahallati, A., 2003, "Aerodynamic of a Low-Pressure Turbine Airfoil under Steady and Periodically Unsteady Conditions," PhD thesis, Carleton University, Ottawa, Canada.

McAuliffe, B. R., 2003, "An Experimental Study of Flow Control Using Blowing for a LowPressure Turbine Airfoil," MASc thesis, Carleton University, Ottawa, Canada.

Müller, R., Vogeler, K., Sauer, H. and Hoeger, M., 2004, "Endwall Boundary Layer Control in Compressor Cascades,” ASME Paper No. GT-2004-53433, Vienna, Austria, June 14-17.

Muthanna, C., 2002, "The Effect of Free Stream Turbulence on the Flow Field through a Compressor Cascade," PhD thesis, Virginia Polytechnic Institute and State University, Blacksburg, Virginia, USA.

Popovic, I., 2005, "Measured Steady and Unsteady Aerodynamic Performance of a Family of Three Highly-Loaded Low-Pressure Turbine Cascades," MASc thesis, Carleton University, Ottawa, Canada.

Praisner, T. J., Allen-Bradley, E., Grover, E. A., Knezevici, D. C. and Sjolander, S. A., 2007, "Application of Non-Axisymmetric Endwall Contouring to Conventional and High-Lift Turbine Airfoils,” ASME Paper No. GT2007-27579, Montreal, QC, CA, May 14-17.

Reising, S. and Schiffer, H.-P., 2009a, "Non-Axisymmetric End Wall Profiling in Transonic Compressors. Part I: Improving the Static Pressure Recovery at Off-Design Conditions by Sequential Hub and Shroud End Wall Profiling," ASME Paper No. GT-2009-59133, Orlando, Florida, USA, June 8-12.

Reising, S. and Schiffer, H.-P., 2009b, "Non-Axisymmetric End Wall Profiling in Transonic Compressors. Part Ii: Design Study of a Transonic Compressor Rotor Using NonAxisymmetric End Walls - Optimization Strategies and Performance," ASME Paper No. GT-2009-59134, Orlando, Florida, USA, June 8-12.

Rodger, P., 1992, "Establishing Tow-Dimensional Flow in a Large-Scale Planar Turbine Cascade," M.Eng. thesis, Carleton University, Ottawa, Canada. 
Rose, M. G., 1994, "Non-Axisymmetric Endwall Profiling in the Hp Ngv's of an Axial Flow Gas Turbine," ASME Paper No. 94-GT-249, Hague, Netherlands, June 13-16.

Schreiber, H.-A., Steinert, W. and Küsters, B., 2002, "Effects of Reynolds Number and FreeStream Turbulence on Boundary Layer Transition in a Compressor Cascade," ASME Journal of Turbomachinery, 124, pp. 1.

Scribner, C. A., 2011, "The Effect of Turbulence Intensity and Reynolds Number on the Aerodynamic Behaviour of Kiel, Three-Hole and Seven-Hole Pressure Probes," MASc thesis, Carleton University, Ottawa, Canada.

Sieverding, C. H., 1985, "Recent Progress in the Understanding of Basic Aspects of Secondary Flows in Turbine Blade Passages," ASME Journal of Engineering for Gas Turbines and Power, 107, pp. 248-257.

Sjolander, S. A., 2012, "Supplementry Course Notes: Turbomachinery " Department of Mechanical and Aerospace Engineering, Carleton University, Ottawa, ON, Canada.

Sonoda, T., Schreiber, H.-A. and Arima, T., 2008, "Endwall Performance of Outlet Guide Vane Cascades with Different Blade Loading Distributions," ASME Paper No. GT2008-51111, Berlin, Germany, June 9-13.

Takeishi, K., Matsuura, M., Aoki, S. and Sato, T., 1990, "An Experiment Study of Heat Transfer and Film Cooling on Low Aspect Ratio Turbine Nozzles," ASME Journal of Turbomachinery, 112, pp. 488-496.

Taremi, F. and Sjolander, S. A., 2011, "Application of Endwall Contouring to Transonic Turbine Cascades: Experimental Measurments at Design Conditions," ASME Paper No. GT201146511, Vancouver, British Columbia, Canada, June 6-10.

Taremi, F., Sjolander, S. A. and Praisner, T., 2010, "Measurements of Endwall Flows in Transonic Linear Turbine Cascades: Part Ii - High Flow Turning," ASME Paper No. GT2010-22760, Glasgow, UK, June 14-18.

Wisler, D. C., Bauer, R. C. and Oklishi, T. H., 1987, "Secondary Flow, Turbulent Diffusion, and Mixing in Axial-Flow Compressors," ASME Journal of Turbomachinery, 109, pp. 455.

Yaras, M. and Sjolander, S. A., 1989, "Losses in the Tip-Leakage Flow of a Planar Cascade of Turbine Blades," Secondary Flows in Turbomachines, AGARD-CP-469, Paper 20, pp. $1-13$.

Yaras, M. and Sjolander, S. A., 1990, "Development of the Tip-Leakage Flow Downstream of a Planar Cascade of Turbine Blades: Vorticity Field," ASME Journal of Turbomachinery, 112, pp. 609-617.

Yaras, M. I., 1987, "Flow in the Tip Gap of a Rectilinear Turbine Cascade," MASc. thesis, Carleton University, Ottawa, Canada. 
Zoric, T., 2006, "Experimental Investigation of Secondary Flows in a Family of Three Highly Loaded Low-Pressure Turbine Cascades," MASc thesis, Carleton University, Ottawa, Canada.

Zoric, T., Popovic, I., Sjolander, S. A., Praisner, T. and Grover, E., 2007a, "Comparative Investigation of Three Highly Loaded Lp Turbine Airfoils: Part I - Measured Profile and Secondary Losses at Design Incidence," ASME Paper No. GT2007-27537, Montreal, QC, Canada, May 14-17.

Zoric, T., Popovic, I., Sjolander, S. A., Praisner, T. and Grover, E., 2007b, "Comparative Investigation of Three Highly Loaded Lp Turbine Airfoils: Part Ii - Measured Profile and Secondary Losses at Off-Design Incidence," ASME Paper No. GT2007-27538, Montreal, QC, CA, May 14-17. 


\section{Appendix A}

\section{Calibration Coefficients for Pressure Transducers}

This appendix outlines the method followed to determine the calibration for the pressure transducers used in the present experiment. The resulting calibration coefficients and sample calibration curves for the DRAL501DN and DRAL505DN transducers are presented in this appendix.

As discussed in Section 3.6.2, the transducer output voltages vary linearly with the applied pressure. The calibration method used is as described by Benner (2003). It consists on applying pressure to the differential pressure transducers using a water micromanometer. Pressures were applied to cover the operating range of the transducers. For each data point, the height difference between the two water columns $(\Delta \mathrm{h})$ and the voltages read by each transducer were recorded and linear relationships were derived from the results. Sample calibration data from the DRAL501DN and DRAL505DN transducers are presented in Figure A.1 and Figure A.2 respectively.

Linear regression analysis was used to determine the slope $\left(\mathrm{C}_{1}\right)$ and the zero pressure voltage offset $\left(\mathrm{C}_{2}\right)$ of each pressure transducers. The resulting coefficients for the DRAL501DN and DRAL505DN transducers are shown in Table A.1 and Table A.2, respectively, along with the correlation coefficient $\left(R^{2}\right)$ showing the goodness of the fit. During the testing in the wind tunnel, for each run performed, one data point was recorded before the start of the test and one was recorded after the end of the test with the wind off in order to correct the zero voltage offset $\left(\mathrm{C}_{2}\right)$ of the calibration curves with the ambient conditions. 


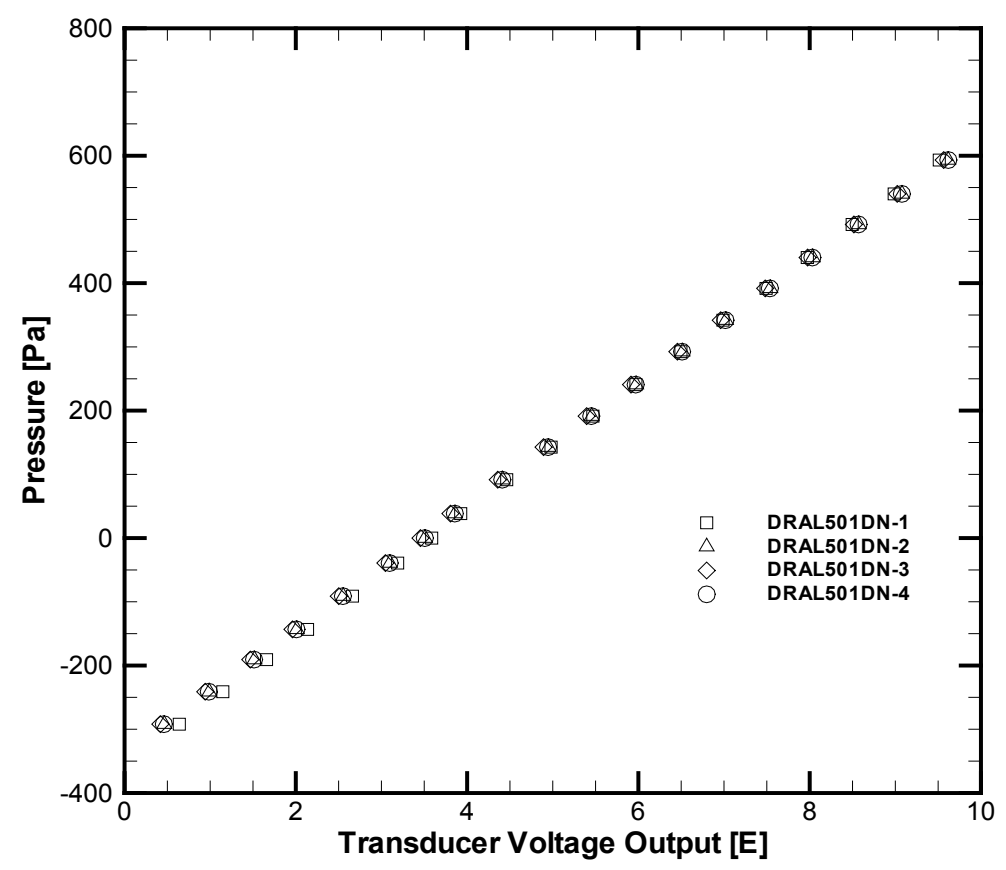

Figure A.1: Sample calibration curves for DRAL501DN pressure transducers

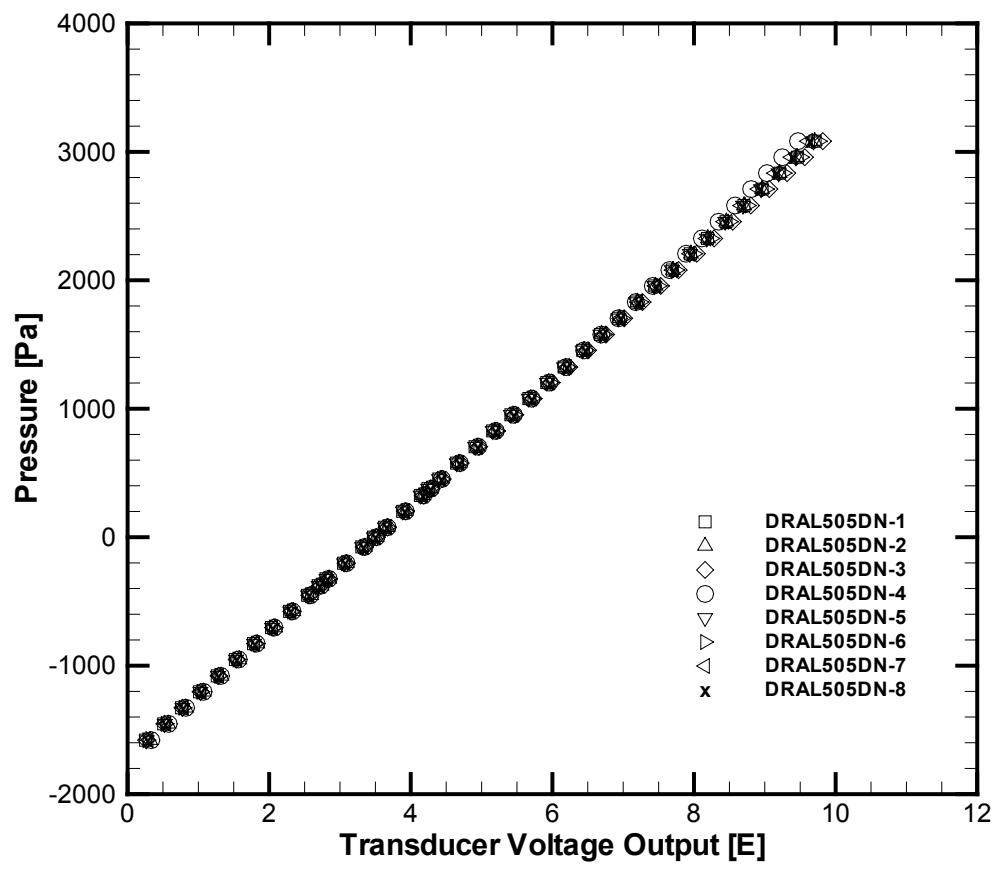

Figure A.2: Sample calibration curves for DRAL505DN pressure transducers 
Table A.1: DRAL501DN calibration coefficients

\begin{tabular}{lccc}
\hline \hline & $\mathbf{C 1}$ & $\mathbf{C 2}$ & $\mathbf{R}^{\mathbf{2}}$ \\
\hline DRAL501DN-1 & 99.83269 & -355.86141 & 0.99998 \\
DRAL501DN-2 & 96.74035 & -337.25316 & 0.99998 \\
DRAL501DN-3 & 96.86066 & -332.55087 & 0.99998 \\
DRAL501DN-4 & 96.71819 & -336.75725 & 0.99998 \\
\hline \hline
\end{tabular}

Table A.2: DRAL505DN calibration coefficients

\begin{tabular}{lccc}
\hline \hline & $\mathbf{C 1}$ & $\mathbf{C 2}$ & $\mathbf{R}^{\mathbf{2}}$ \\
\hline DRAL505DN-1 & 492.33212 & -1707.07011 & 0.99999 \\
DRAL505DN-2 & 495.96692 & -1739.48659 & 0.99999 \\
DRAL505DN-3 & 487.06827 & -1708.83759 & 0.99999 \\
DRAL505DN-4 & 504.20338 & -1765.20120 & 0.99962 \\
DRAL505DN-5 & 493.90351 & -1714.77255 & 0.99999 \\
DRAL505DN-6 & 490.58259 & -1719.20545 & 0.99999 \\
DRAL505DN-7 & 496.69724 & -1735.67147 & 0.99986 \\
DRAL505DN-8 & 494.86203 & -1728.33912 & 0.99988 \\
\hline \hline
\end{tabular}




\section{Appendix B}

\section{Calibration and Data Reduction Procedures for Three-Hole Probe}

This appendix describes the procedures followed for the calibration and data reduction of the three-hole pressure probe used in this experiment. The pressure probe, introduced in Section 3.8.2, was calibrated in the calibration apparatus described in Section 3.7 over a range of pitch angle $\beta= \pm 21^{\circ}$ in increments of $0.5^{\circ}$. The probe was calibrated at two different velocities $(15 \mathrm{~m} / \mathrm{s}$ and $25 \mathrm{~m} / \mathrm{s})$ corresponding to the cascade outlet and inlet nominal velocities, respectively.

The jet of the calibration apparatus is well documented in Section 3.8.2 of Mahallati's thesis (Mahallati, 2003). The jet calibration coefficients were extracted from his results for the current probe tip location downstream of the jet nozzle. The jet calibration coefficients for total pressure $\left(C_{P 0, c a l}\right)$, static pressure $\left(C_{P s, c a l}\right)$ and dynamic pressure $\left(C_{q, c a l}\right)$ are defined as:

$$
\begin{aligned}
C_{P 0, c a l} & =\frac{P_{0}-P_{C 1}}{P_{C 1}-P_{C 2}} \\
C_{P s, c a l} & =\frac{P_{s}-P_{C 2}}{P_{C 1}-P_{C 2}} \\
C_{q, c a l} & =\frac{P_{0}-P}{P_{C 1}-P_{C 2}}=1+C_{P 0}-C_{P s}
\end{aligned}
$$


The first step of the calibration is to aerodynamically align the pressure probe with the jet flow. Once aligned, the probe is rotated around its pitch axis (refer to Figure 3.7) over the range of angles specified previously and calibration data are recorded at each position. The probe was only rotated around its pitch axis as it has been shown that the probe is insensitive to yaw misalignments up to $\pm 5^{\circ}$ (Mahallati, 2003). For each measurement angle, the pressures recorded at each probe port $\left(\mathrm{P}_{h}\right)$ are corrected for the nozzle calibration using the following equation:

$$
C_{P h}=\frac{\frac{P_{h}-P_{C 2}}{P_{C 1}-P_{C 2}}-C_{P s, c a l}}{C_{q, c a l}} \quad \text { for } h=1,2,3
$$

Then, the method of Lewis (1966) is used to determine the following non-dimensional calibration coefficients of the three-hole pressure probe based on the corrected pressures:

$$
\begin{aligned}
& K_{1}=\frac{P_{1}-P}{P_{0}-P}=C_{P 1} \\
& K_{12}=\frac{P_{1}-P_{2}}{P_{0}-P}=C_{P 1}-C_{P 2} \\
& K_{13}=\frac{P_{1}-P_{3}}{P_{0}-P}=C_{P 1}-C_{P 3} \\
& K_{23}=\frac{P_{2}-P_{3}}{P_{0}-P}=C_{P 2}-C_{P 3} \\
& K_{\phi 2}=\frac{P_{1}-P_{2}}{P_{1}-P_{3}}=\frac{K_{12}}{K_{13}} \\
& K_{\phi 3}=\frac{P_{1}-P_{2}}{P_{1}-P_{3}}=\frac{K_{12}}{K_{13}}
\end{aligned}
$$

The calibration curves used in this experiment are shown on Figure B.1 for both the inlet and outlet nominal velocities of the cascade. As observed, the jet velocity, and thus the Reynolds number, seems to have some effect on some of the calibration coefficients at high pitch angles, in particular $K_{12}$ and $K_{13}$. Since the three-hole probe shows to be slightly sensitive to Reynolds number, both sets of calibration curves were used, depending on the location of the probe (upstream or downstream of the cascade). More details on the threehole probe sensitivity to Reynolds number are available in Appendix B of McAuliffe (2003). 

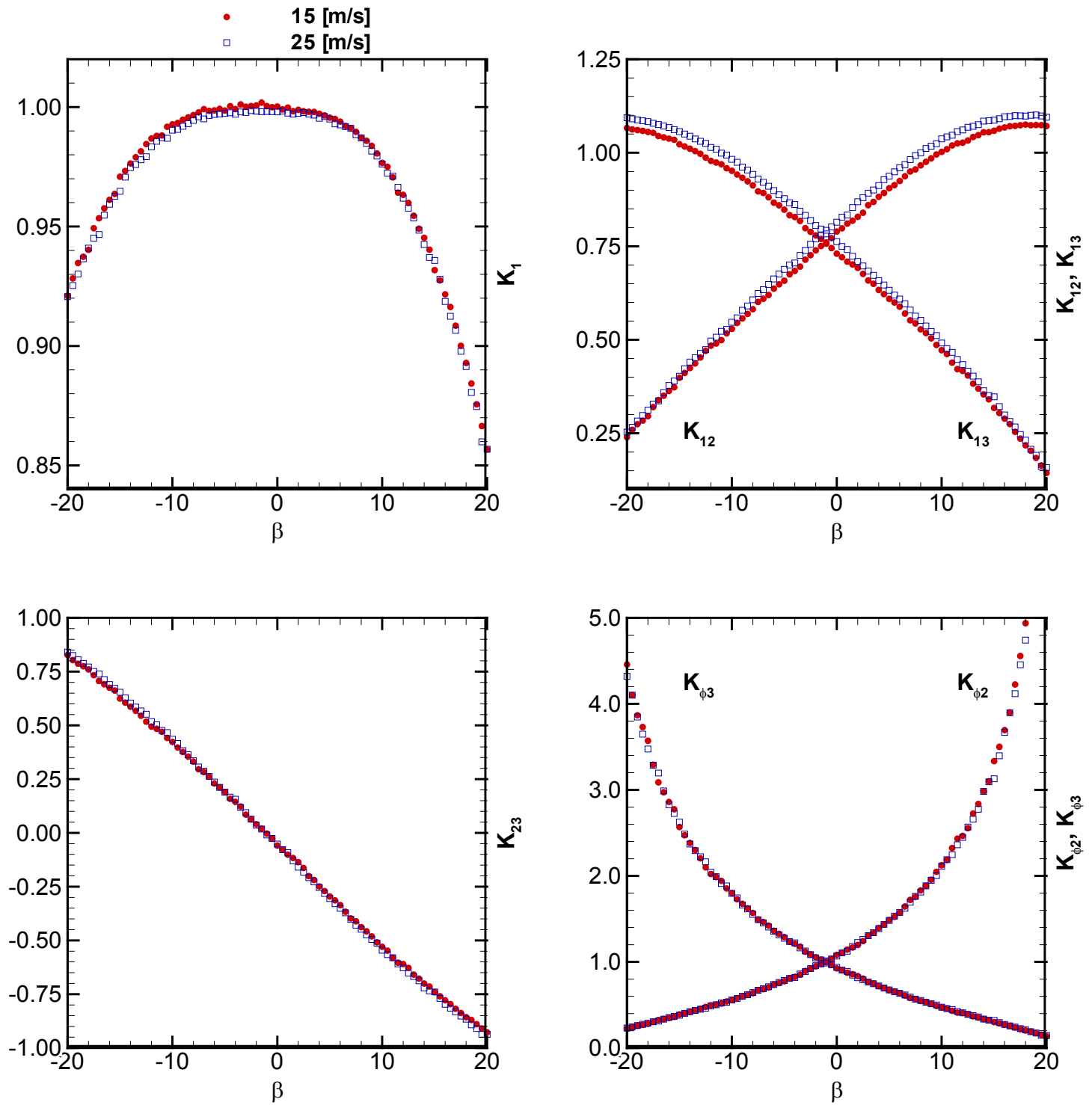

Figure B.1: Three-hole probe calibration curves

With the probe calibrated, the following procedure is used to determine the flow angle as well as the total, static and dynamic pressures in an unknown flow field. The first step consists on determining the sign of the flow angle by comparing $P_{2}$ and $P_{3}$. If the port $P_{2}$ reads higher, the flow angle is negative. As observed on Figure B.1, the scatter is less for the $K_{\phi 2}$ data at negative angles, such that the $K_{\phi 2}$ calibration curve is used to determine the flow angle $\beta$. Otherwise, if the port $P_{3}$ reads higher, the flow angle is positive and the calibration 
curve $K_{\phi 3}$ is used to determine the flow misalignment. Once the flow misalignment is determined, either $K_{12}$ or $K_{13}$ calibration coefficients is used to calculate the local dynamic pressure $q$. The coefficient used is based on the sign of the flow angle. Finally, knowing the local dynamic pressure, the flow misalignment and the calibration coefficient $K_{l}$, the local static pressure $P$ is determined. The local total pressure can then be calculated using $P$ and $q$. 


\section{Appendix C}

\section{Calibration and Data Reduction Procedures for Seven-Hole Probe}

This appendix describes the procedure followed for the calibration and data reduction of the goose-neck seven-hole probe (GN7HP) used in this experiment. The pressure probe was introduced in Section 3.8.3 and is shown schematically on Figure 3.8. The seven-hole probe was calibrated in the apparatus described in Section 3.7 over a pitch $(\beta)$ and yaw $(\alpha)$ angles range of $-50^{\circ}<\alpha, \beta>50^{\circ}$ in $2^{\circ}$ increments. It was calibrated at nine different jet flow velocities $(5,10,15,20,25,30,35,40$ and $45 \mathrm{~m} / \mathrm{s})$ to cover the range of probe Reynolds numbers, based on tip width, seen in the experiment.

The calibration and data reduction procedures used in this research were developed by MacIsaac et al. (2009) and by the present author, adapted from the methods of Benner (2003) and Gerner et al. (1984). The data reduction procedure was modified to add an iterative process to interpolate between the calibration data at different Reynolds number for the local Reynolds number experienced by the probe. The procedure was also adapted to allow extrapolation of the calibration data for a local Reynolds number outside of the calibrated range.

The calibration and data reduction procedures are divided into two regimes. The first regime is the low-angle regime, where the central port of the probe (7) reads the highest pressure. The second regime is the high-angle regime, where one of the surrounding ports (16) reads the highest pressure. Each regime has a different procedure, which will be described below. The division of angular space for the tip of the probe is shown on Figure C.1. 


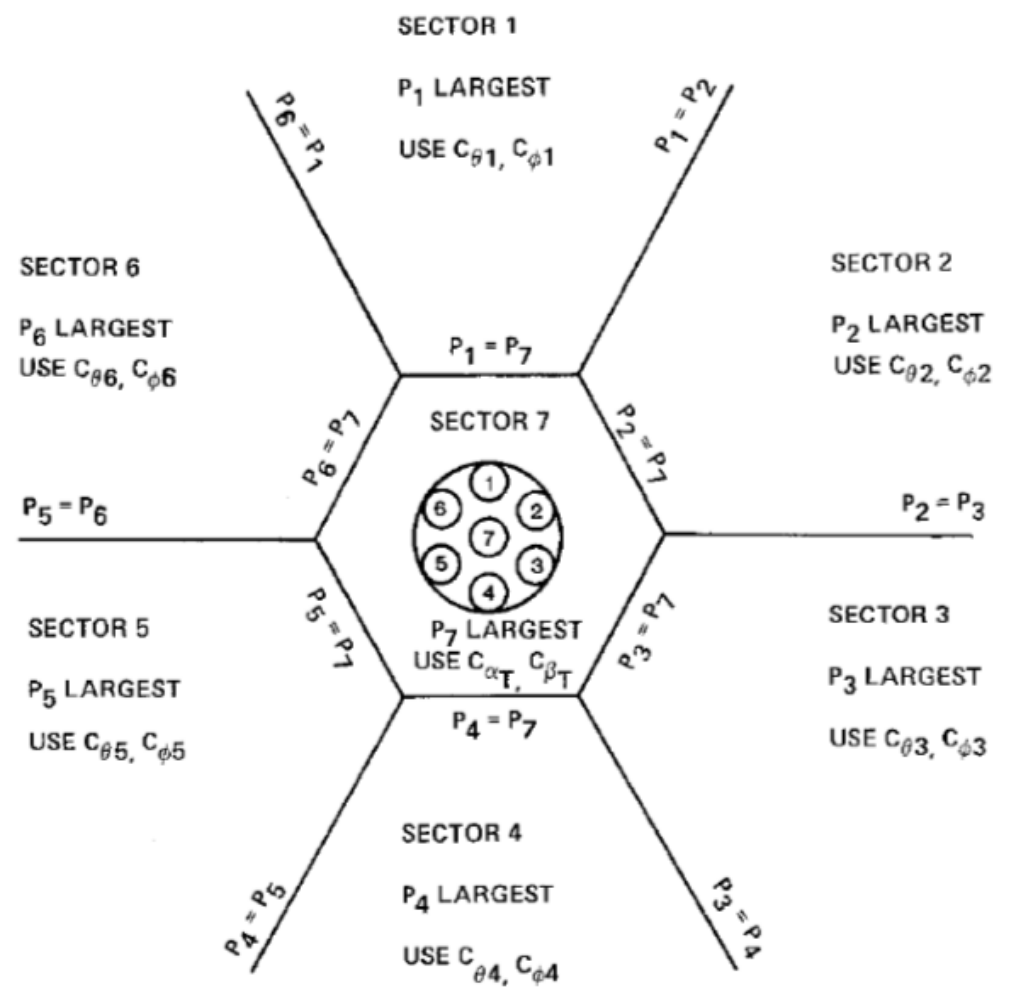

Figure C.1: Division of angular space (reproduced from Gerner et al. (1984))

\section{Low-Angle Regime}

For small flow misalignments, typically on the order of $\pm 20^{\circ}$ relative to the probe tip, the flow is expected to remain attached on the entire surface of the probe tip. In these cases, the central port (port 7) senses the highest pressure. Pressure information from all seven ports is used to define the following non-dimensional coefficients in the low-angle regime:

$$
\begin{gathered}
C_{\alpha 1}=\frac{P_{4}-P_{1}}{P_{7}-\bar{P}_{1-6}} \\
C_{\alpha 2}=\frac{P_{3}-P_{6}}{P_{7}-\bar{P}_{1-6}} \\
C_{\alpha 3}=\frac{P_{2}-P_{5}}{P_{7}-\bar{P}_{1-6}}
\end{gathered}
$$

In the above equations, $\bar{P}_{1-6}$ corresponds to the average of the six surrounding pressure ports and it approximates the local static pressure. The local total pressure is approximated by 
$P_{7}$, which means that the denominator, $P_{7}-\bar{P}_{1-6}$, is referred as the pseudo local dynamic pressure. The numerators are formed from opposite ports of the probe, such that each coefficient is most sensitive to angular misalignment on one of the three planes of the probe separated by $60^{\circ}$.

The coefficients defined in Equation C. 1 are used to define two angular coefficients, $C_{\alpha}$ and $C_{\beta}$, for the tangential coordinate system shown below on Figure C.2.

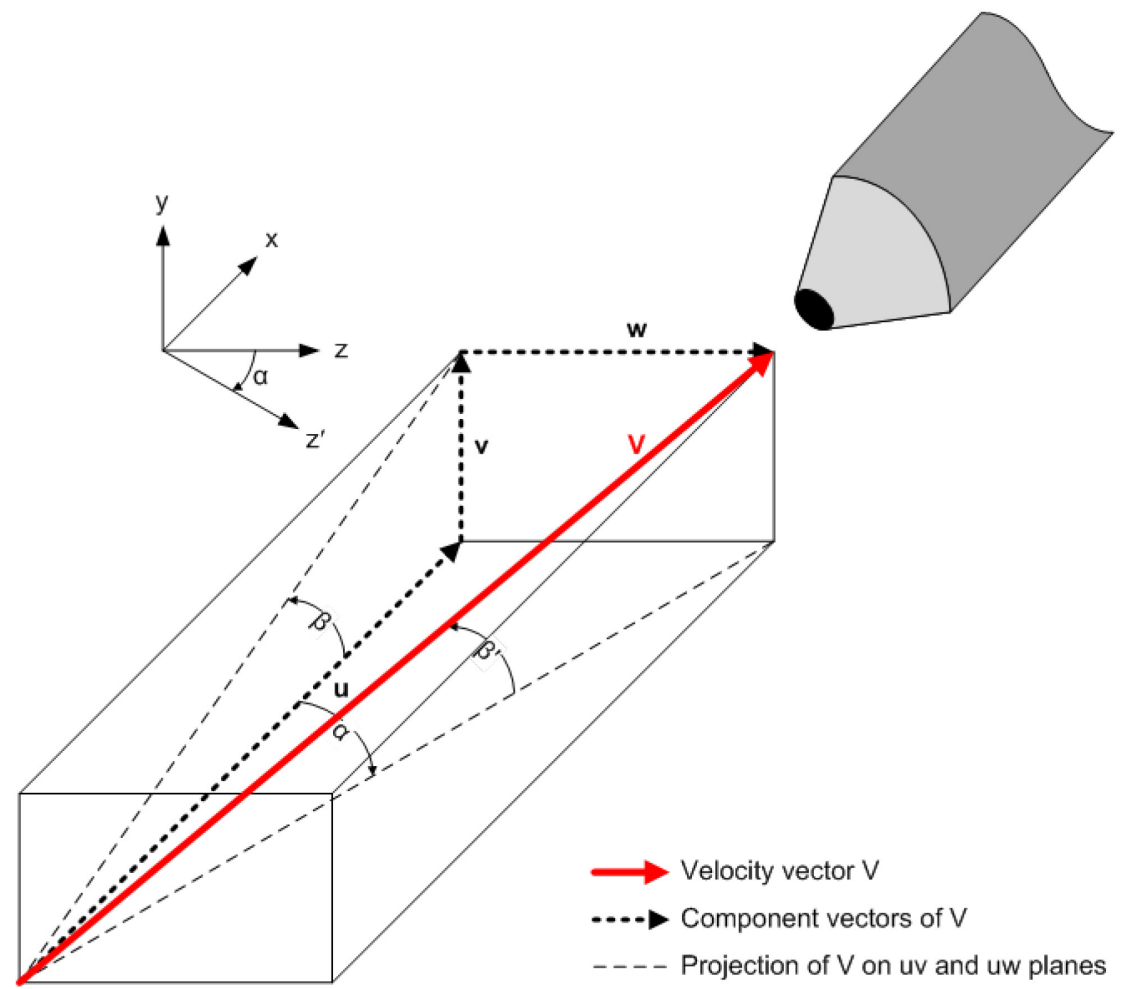

Figure C.2: Probe Tangential Coordinate System (adapted from Gerner et al., 1984)

$$
\begin{aligned}
& C_{\alpha}=\frac{2 C_{\alpha 1}+C_{\alpha 2}-C_{\alpha 3}}{3} \\
& C_{\beta}=\frac{C_{\alpha 2}+C_{\alpha 3}}{\sqrt{3}}
\end{aligned}
$$

On the tangential coordinate system shown above, $\alpha$ is measured from the probe $\mathrm{x}$-axis ( $u$ component of velocity) to the projection of $\boldsymbol{V}$ on the xz-plane and is positive when port 4 is reading the highest. Similarly, $\beta$ is the angle between the $\mathrm{x}$-axis of the probe to the projection of $\boldsymbol{V}$ on the xy-plane and is positive when ports 2 and 3 read the highest. It should be noted that, in the calibration apparatus, the probe pitch control is coupled to the yaw 
control. The yaw turntable is fixed to the ground and always rotates around the y-axis shown in the figure above, such that it independently varies $\alpha$. However, the pitch turntable is fixed to the yaw turntable, such that it rotates around the $z^{\prime}$-axis and the corresponding angle is defined as $\beta^{\prime}$. Thus, the tangential coordinate $\beta$ is a function of both pitch and yaw turntable angles and can be calculated from:

$$
\beta=\tan ^{-1}\left(\frac{\tan \beta^{\prime}}{\cos \alpha}\right)
$$

During the calibration of the probe, the coefficients defined in Equations C.1 and C.2 are calculated for each position in $\alpha$ and $\beta$ of the probe. The total and dynamic pressure coefficients defined below are also calculated to form the calibration tables that will be used for the probe in an unknown flow:

$$
\begin{aligned}
& C_{0}=\frac{P_{7}-P_{0}}{P_{7}-\bar{P}_{1-6}} \\
& C_{q}=\frac{P_{7}-\bar{P}_{1-6}}{P_{0}-P}
\end{aligned}
$$

With the probe calibrated in a known flow, the local pitch angle $(\beta)$, yaw angle $(\alpha)$, total pressure coefficient $\left(C_{0}\right)$ and dynamic pressure coefficient $\left(C_{q}\right)$ can be related to Equation C.2 as follows:

$$
\begin{aligned}
& \alpha=f_{1}\left(C_{\alpha}, C_{\beta}\right) \\
& \beta=f_{2}\left(C_{\alpha}, C_{\beta}\right) \\
& C_{0}=f_{3}\left(C_{\alpha}, C_{\beta}\right) \\
& C_{q}=f_{4}\left(C_{\alpha}, C_{\beta}\right)
\end{aligned}
$$

Sample calibration data are shown for a jet velocity of $15 \mathrm{~m} / \mathrm{s}$ on Figure C.3 to Figure C.6 (corresponding to the nominal outlet velocity of the cascade). Each contour plot shows the calibration data of one of the coefficient to demonstrate the smoothness of the data and corresponds to a look-up table used for data reduction. Similar calibration contours/look-up tables have been generated for other jet velocities in order to take into account the Reynolds number effect on the seven-hole probe. The figures also contain two other plots showing data along lines of $\alpha=0$ and $\beta=0$. 


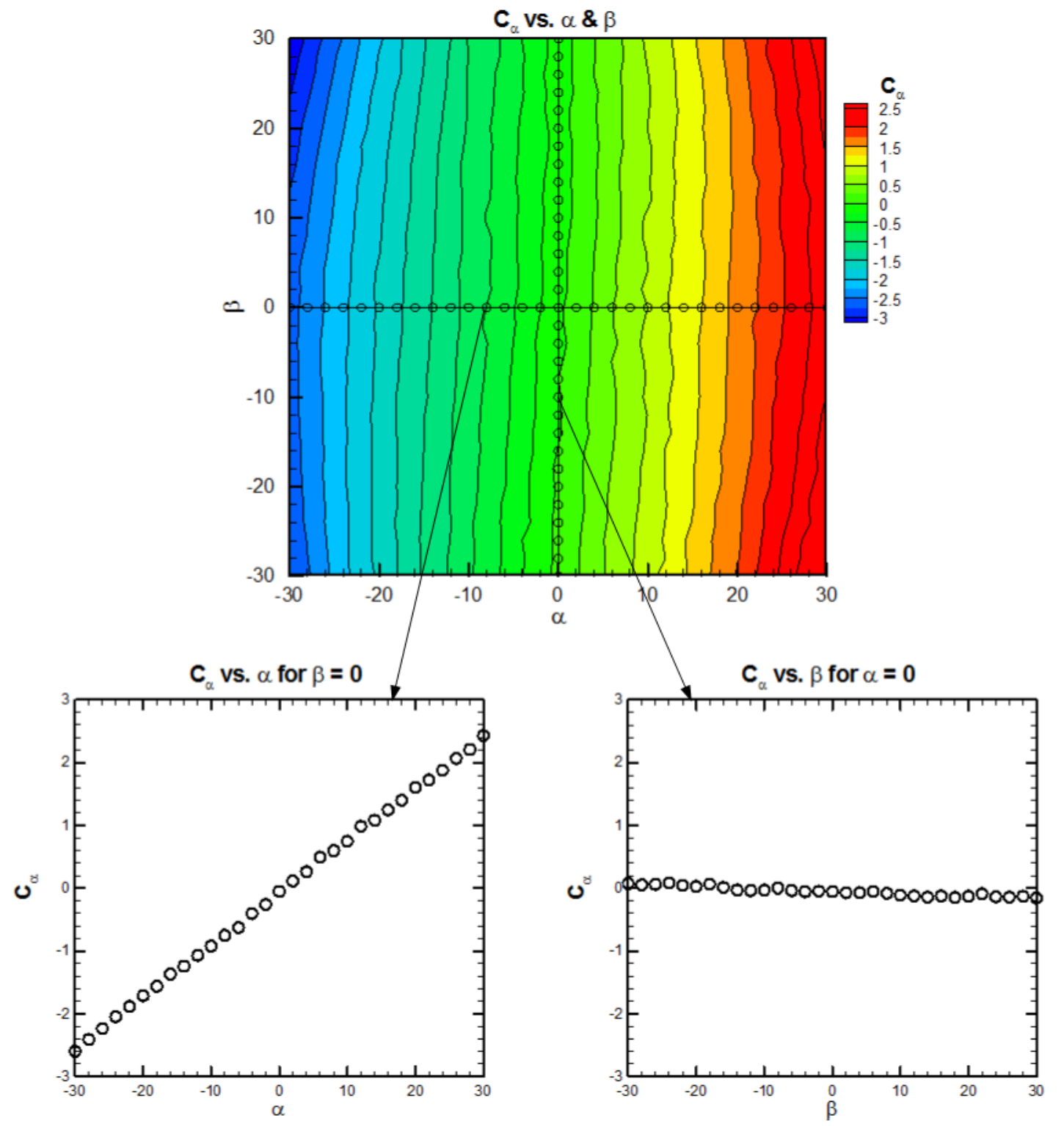

Figure C.3: $C_{\alpha}$ calibration data for sector 7 at $15 \mathrm{~m} / \mathrm{s}$ 


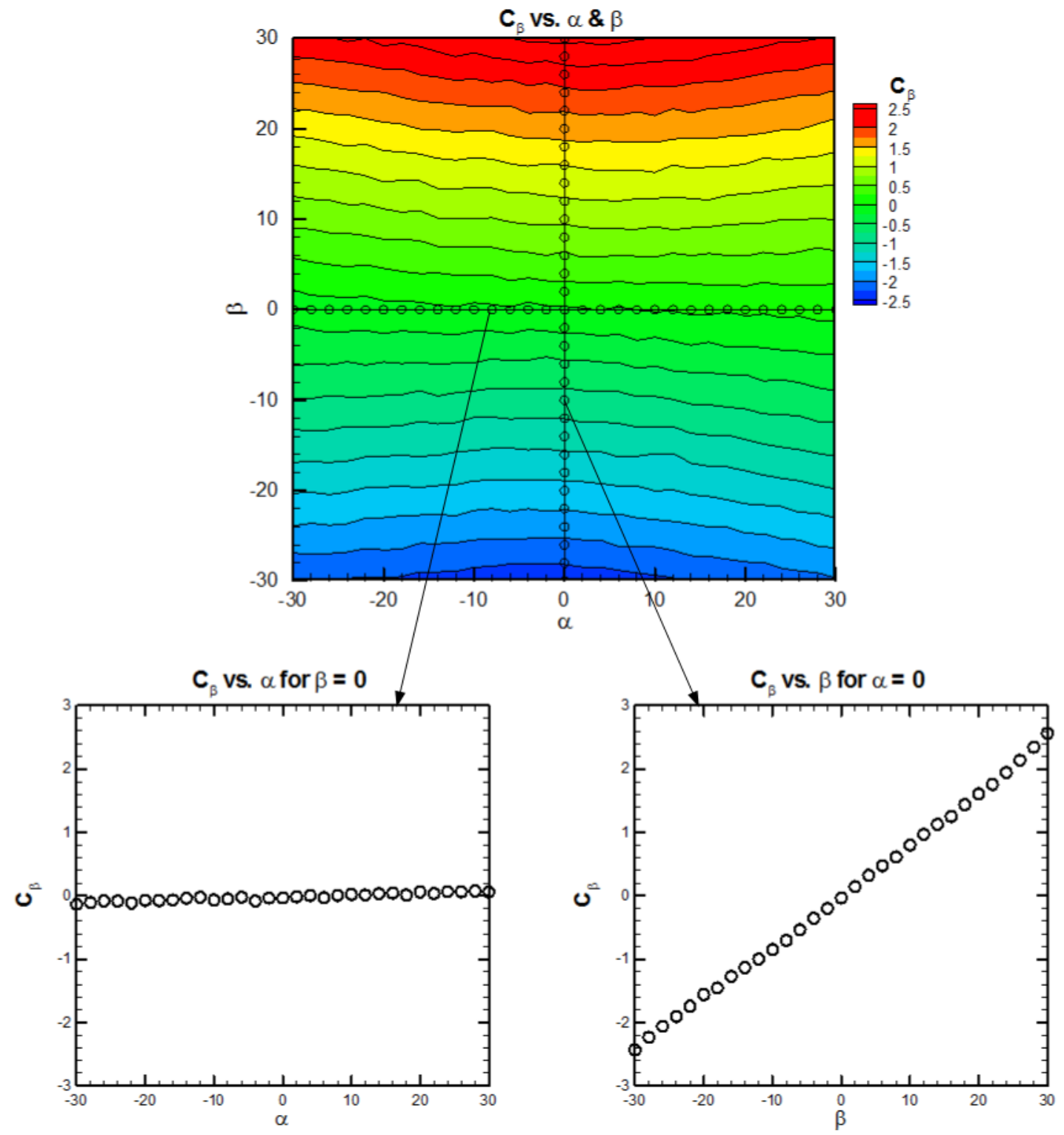

Figure C.4: $C_{\beta}$ calibration data for sector 7 at $15 \mathrm{~m} / \mathrm{s}$ 


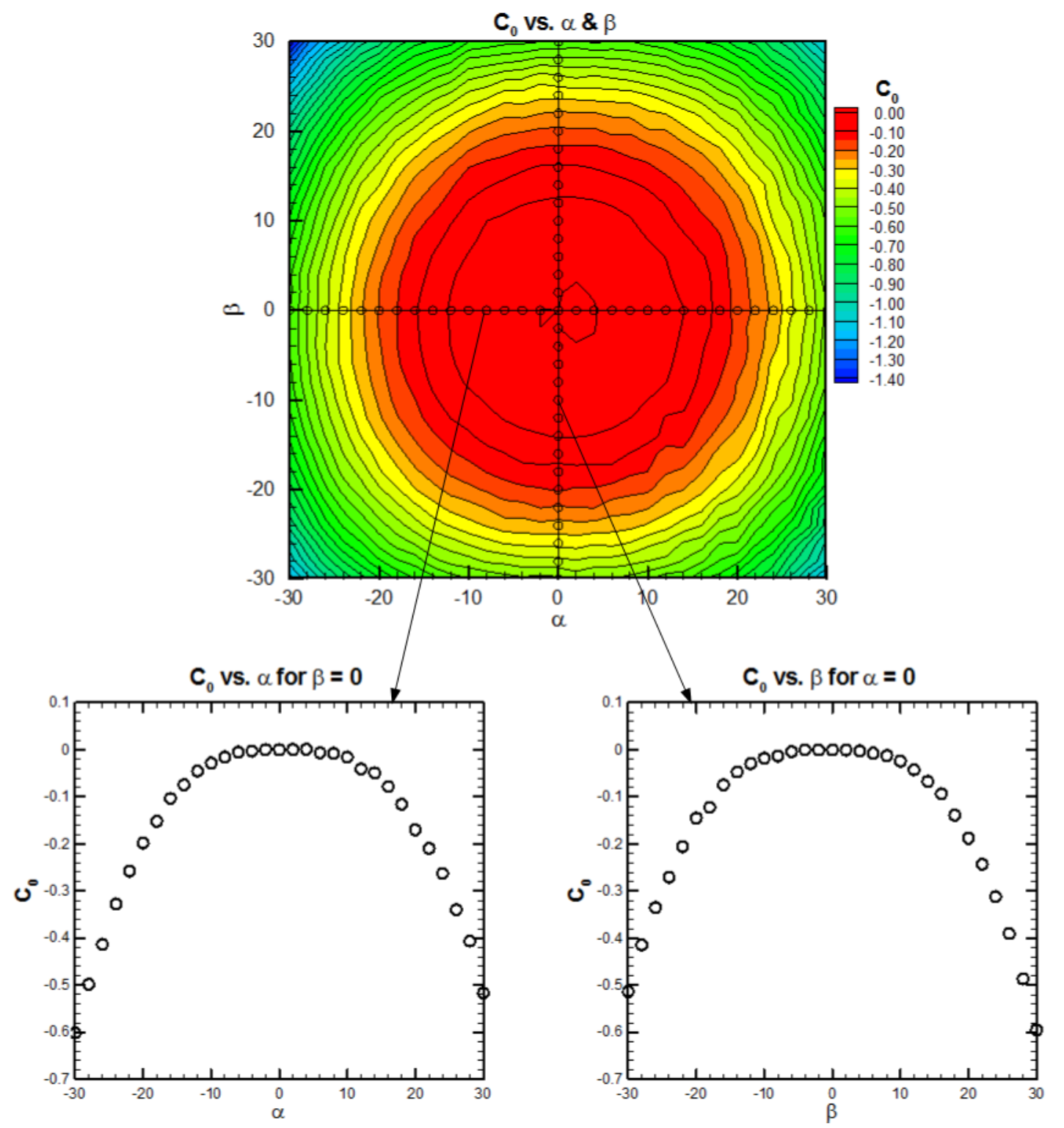

Figure C.5: $C_{0}$ calibration data for sector 7 at $15 \mathrm{~m} / \mathrm{s}$ 


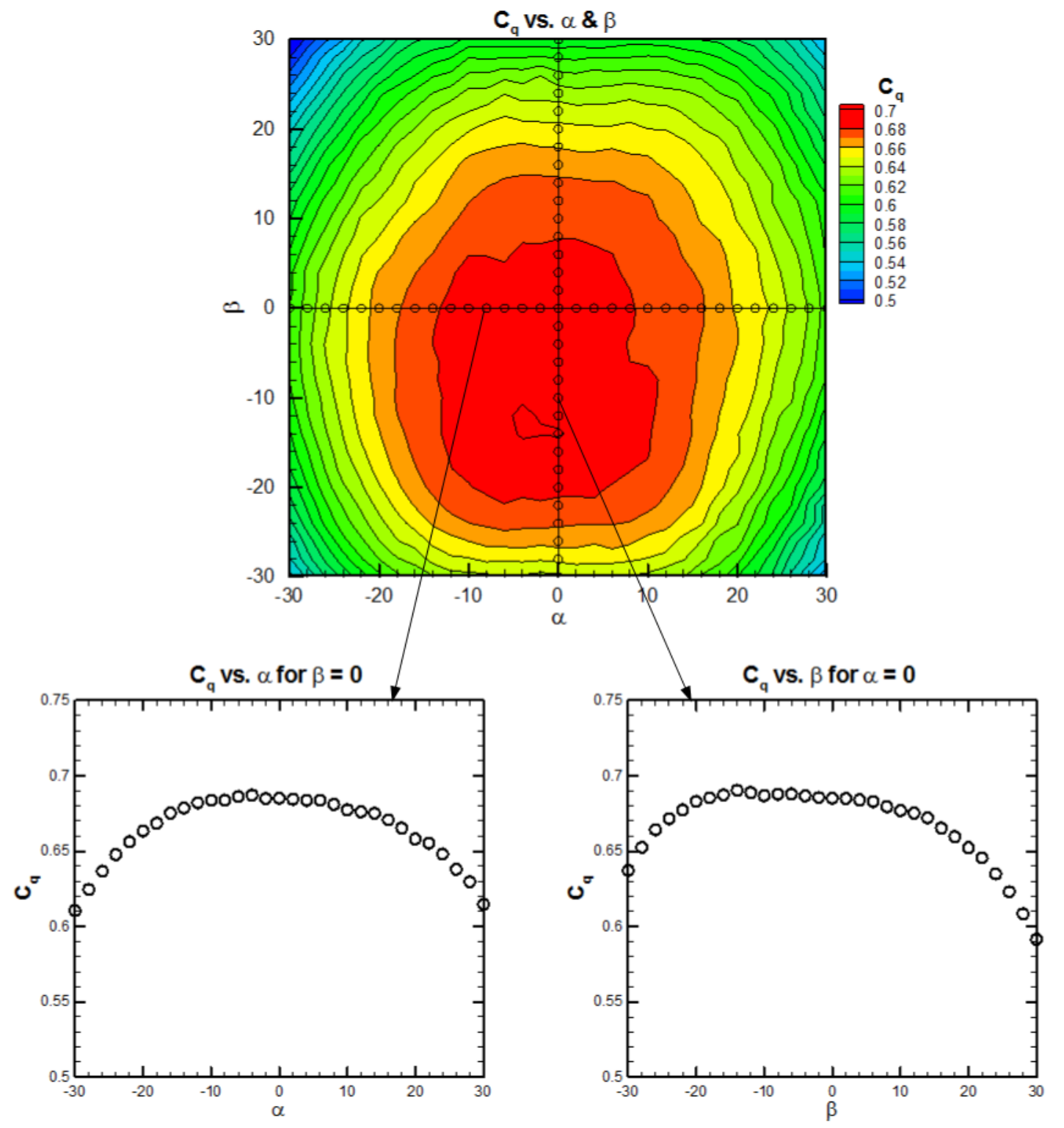

Figure C.6: $C_{q}$ calibration data for sector 7 at $15 \mathrm{~m} / \mathrm{s}$

The data reduction procedure for the low-angle regime uses linear interpolation in look-up tables represented by the contour plots shown above. Look-up tables have been generated for nine Reynolds numbers (based on the jet velocities specified above and on the probe tip diameter). 
In an unknown flow, the procedure consists on interpolating $\alpha, \beta, C_{0}$ and $C_{q}$ based on the angular coefficients $C_{\alpha}$ and $C_{\beta}$ and using the calibration data at the highest calibrated Reynolds number as a first assumption.

Then, using the calibration coefficients $C_{0}$ and $C_{q}$, it is possible to calculate the local total and dynamic pressures from Equation C.4. Using the dynamic pressure, the local Reynolds number is calculated for each data point recorded. Using again $C_{\alpha}$ and $C_{\beta}$, the four parameters defined in Equation C.5 are interpolated/extrapolated for the local Reynolds numbers calculated previously using the calibration tables at the different Reynolds numbers. The local Reynolds numbers are again calculated for each data point using the newly calculated dynamic pressures and the results are compared to the previous values of Reynolds number. This procedure is repeated until the difference between the new local Reynolds number and the old one is below the specified convergence tolerance.

Finally, once the local Reynolds numbers converged below the specified value, the local static pressure and the unit velocity vectors $(u / V, v / V, w / V)$ are calculated for each data point. Thus, using the previously defined data reduction procedure for the low-angle regime, each of the following parameters are recorded for all data points: yaw angle $(\alpha)$, pitch angle $(\beta)$, total pressure coefficient $\left(C_{P 0}\right)$, dynamic pressure coefficient $\left(C_{q}\right)$, static pressure coefficient $\left(C_{P S}\right)$ and the unit velocity vectors $(u / V, v / V, w / V)$. The calibration coefficients $\left(C_{\alpha}, C_{\beta}, C_{0}\right.$ and $\left.C_{q}\right)$ are also recorded.

The procedure described above is used as long as the central port (P7) reads the highest pressure, which is typical for flows between $+/-30^{\circ}$.

\section{High-Angle Regime}

Typically, when the tip of the probe is exposed to large angles, typically, the flow will separate from the opposite surface of the probe tip. Since the separated probe ports are insensitive to change in flow misalignment, it is necessary to define a procedure which only uses the surfaces where the flow remains attached. This procedure is used when one of the six surrounding ports reads the highest pressure.

The data reduction procedure uses a polar coordinate system as suggested by Gallington (1981), which is shown on Figure C.7. The pitch angle, $\theta$, is measured from the 
probe $\mathrm{x}$-axis to the velocity vector $\boldsymbol{V}$ and the roll angle, $\phi$, is measured from the positive zaxis to the projection of $\boldsymbol{V}$ on the yz-plane.

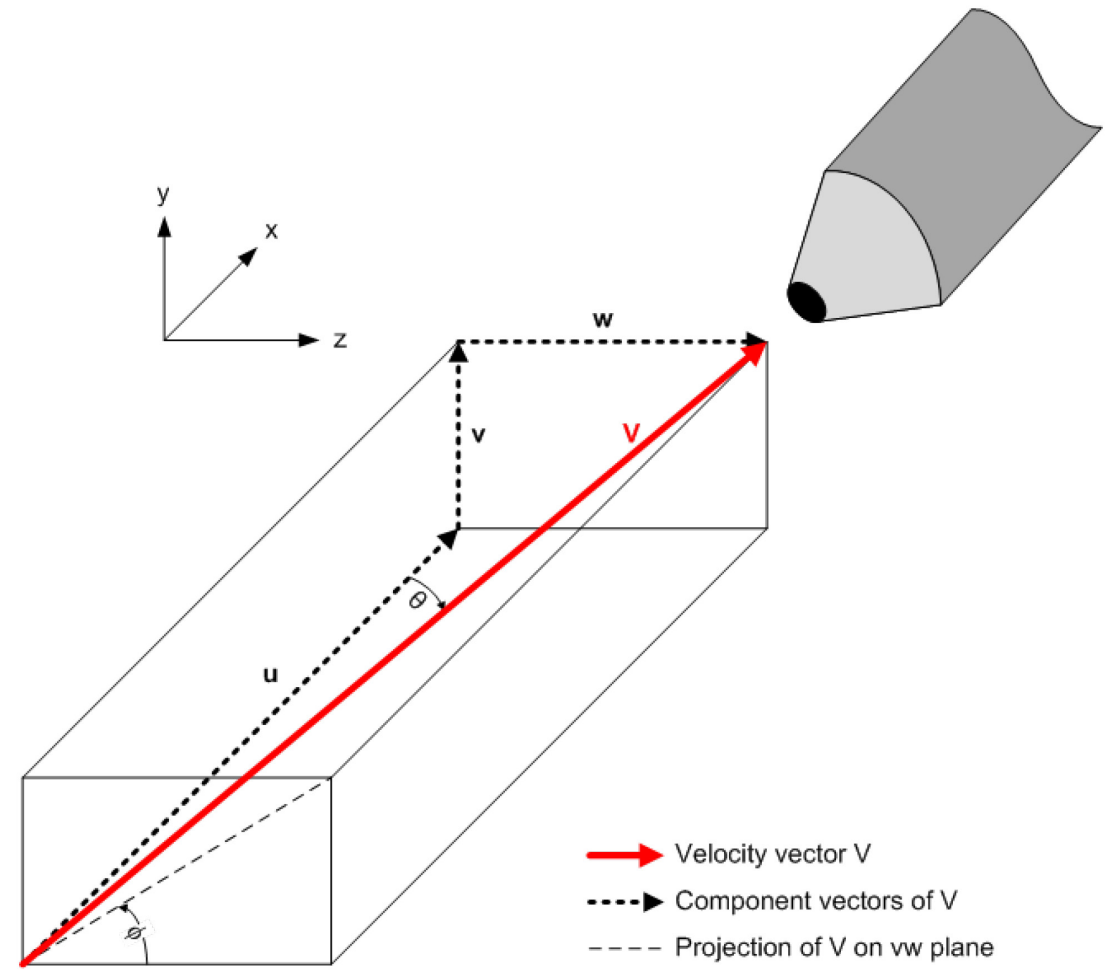

Figure C.7: Probe Polar Coordinate System, adapted from Gerner et al. (1984)

Using the above coordinate system, the following two coefficients are defined for the high-angle regime:

$$
\begin{gathered}
C_{\theta n}=\frac{P_{n}-P_{7}}{P_{n}-\left(\frac{P_{n-}+P_{n+}}{2}\right)} \\
C_{\phi n}=\frac{P_{n-}-P_{n+}}{P_{n}-\left(\frac{P_{n-}+P_{n+}}{2}\right)}
\end{gathered}
$$

The subscript $n$ refers to the peripheral pressure port number reading the highest pressure and the subscripts $n$ - and $n+$ are the adjacent counterclockwise and clockwise peripheral ports, respectively. These two coefficients are defined as the angular sensitivity coefficients. During the calibration of the probe in a known flow, the two coefficients can be related to the pitch angle $(\theta)$, the roll angle $(\phi)$, the total pressure coefficient $\left(\mathrm{C}_{0}\right)$ and the dynamic pressure coefficient $\left(\mathrm{C}_{\mathrm{q}}\right)$ using the following relationships: 


$$
\begin{aligned}
& \theta=f_{1}\left(C_{\theta n}, C_{\phi n}\right) \\
& \phi=f_{2}\left(C_{\theta n}, C_{\phi n}\right) \\
& C_{0}=f_{3}\left(C_{\theta n}, C_{\phi n}\right) \\
& C_{q}=f_{4}\left(C_{\theta n}, C_{\phi n}\right)
\end{aligned}
$$

In this case, the total pressure coefficient and the dynamic pressure coefficient are defined as followed:

$$
\begin{gathered}
C_{0}=\frac{P_{n}-P_{0}}{P_{n}-\left(\frac{P_{n-}+P_{n+}}{2}\right)} \\
C_{q}=\frac{P_{n}-\left(\frac{P_{n-}+P_{n+}}{2}\right)}{P_{0}-P}
\end{gathered}
$$

With the probe calibrated in a known flow for the high-angle regime and with the lookup tables created for each of the nine Reynolds number based on the probe diameter, the data reduction procedure used for the high-angle regime is the same as for the low-angle regime. For each data point, linear interpolation is used between the look-up tables at different Reynolds number to find the two angles and two pressure coefficients defined in Equation C.7 above and the process is repeated until the local Reynolds numbers converged below a specified tolerance. Sample calibration data for the seven-hole probe port 4 are shown on Figure C.8 to Figure C.11. 


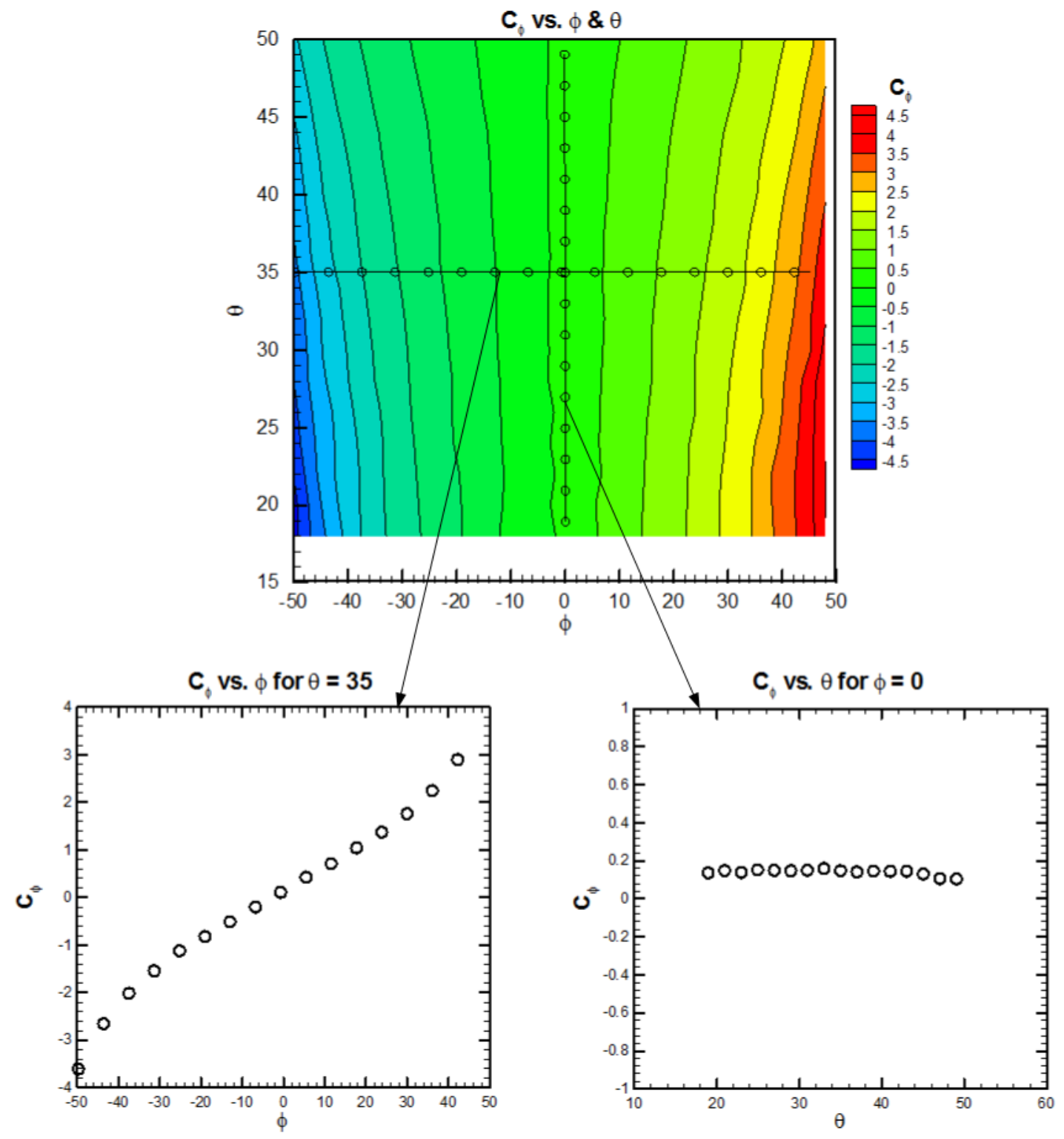

Figure C.8: $C_{\phi}$ calibration data for sector 4 at $15 \mathrm{~m} / \mathrm{s}$ 


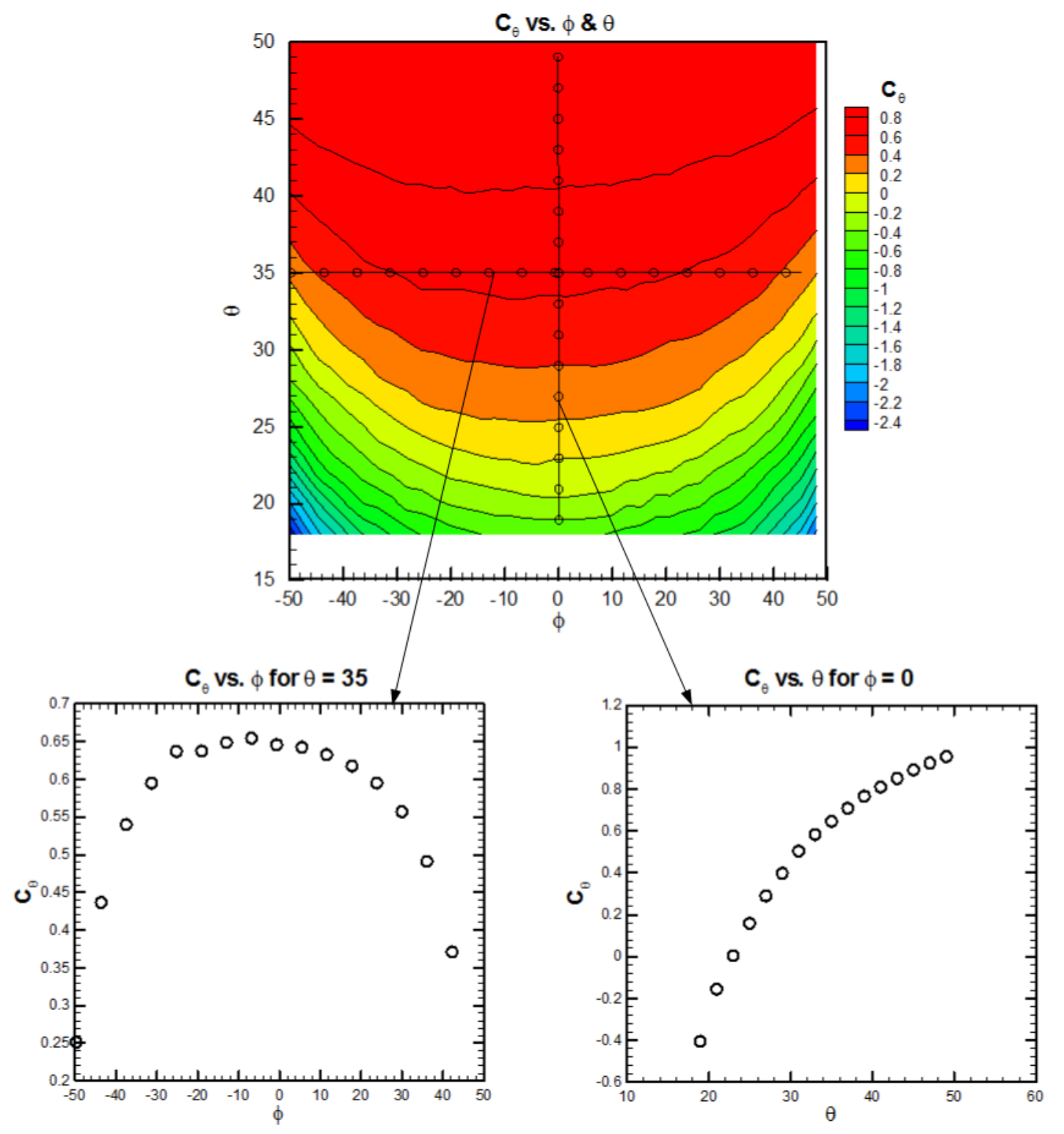

Figure C.9: $C_{\theta}$ calibration data for sector 4 at $15 \mathrm{~m} / \mathrm{s}$ 


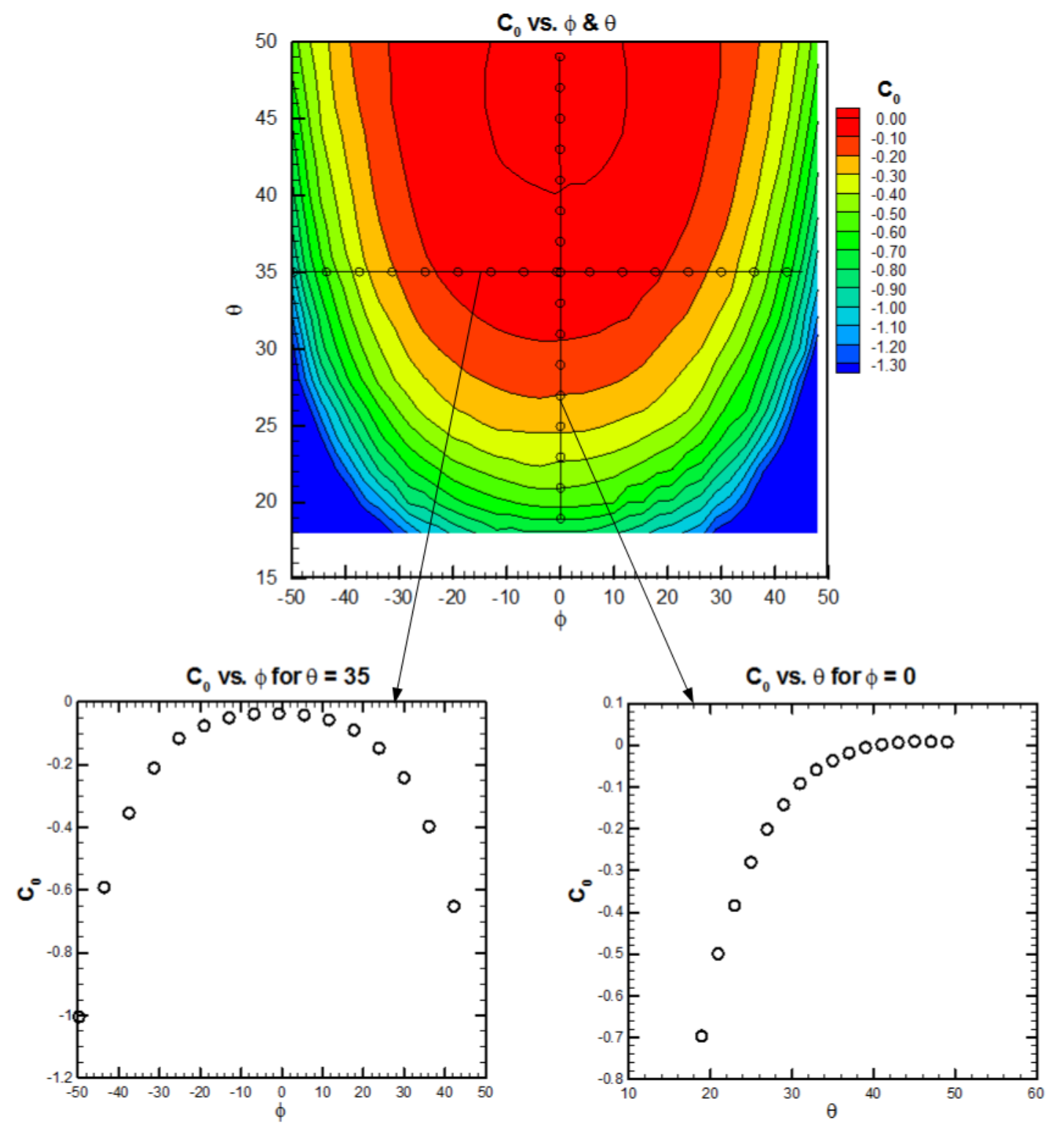

Figure C.10: $C_{0}$ calibration data for sector 4 at $15 \mathrm{~m} / \mathrm{s}$ 


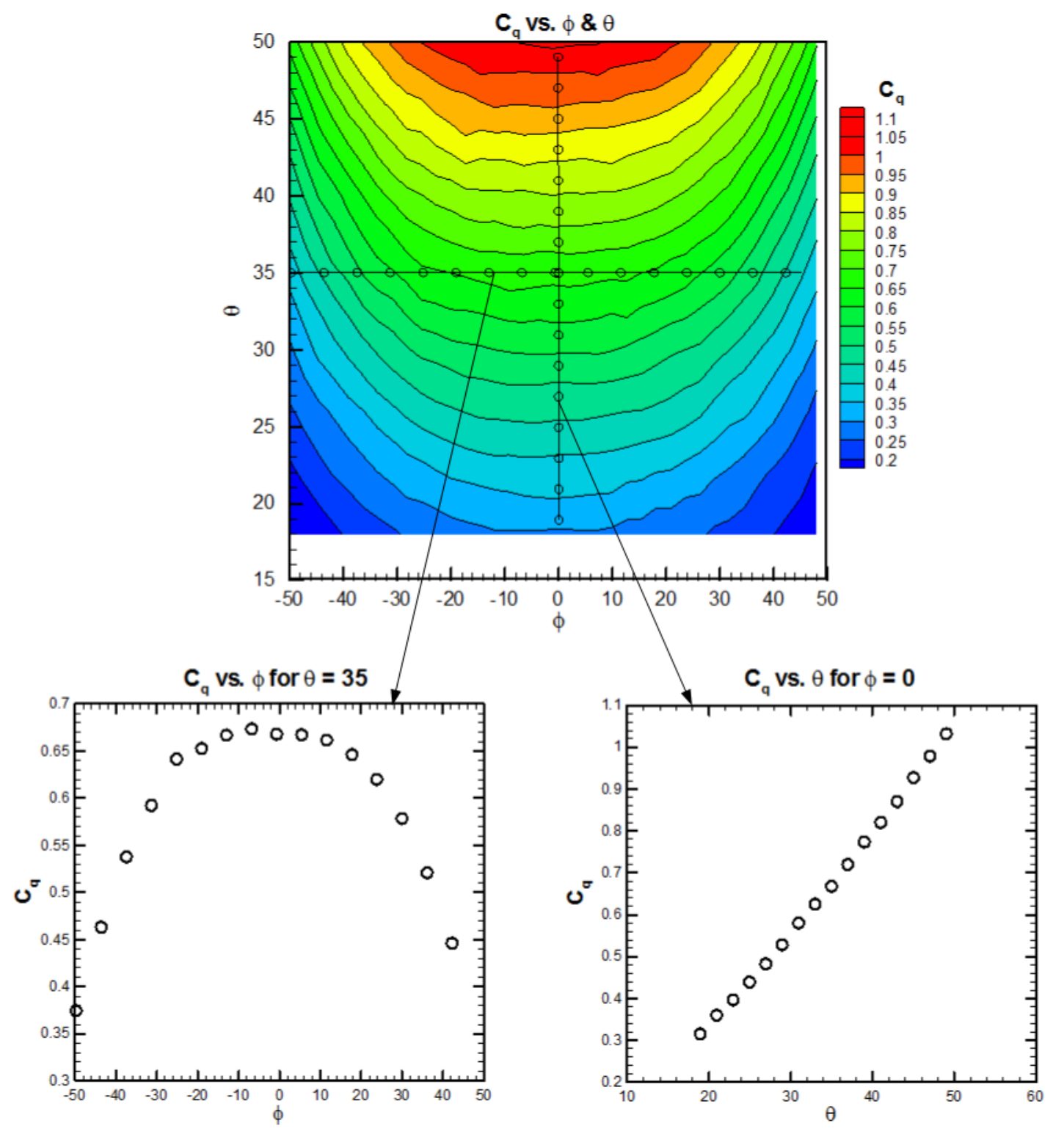

Figure C.11: $C_{q}$ calibration data for sector 4 at $15 \mathrm{~m} / \mathrm{s}$ 